\title{
Instabilities of an Eastern Boundary Current with and without Large-scale Flow Influence
}

by

\author{
Jinbo Wang
}

Submitted in partial fulfillment of the

requirements for the degree of

Doctor of Philosophy in Physical Oceanography

at the

MASSACHUSETTS INSTITUTE OF TECHNOLOGY

and the

WOODS HOLE OCEANOGRAPHIC INSTITUTION

June, 2011

(c) Jinbo Wang 2011. All rights reserved.

The author hereby grants to MIT and to WHOI permission to reproduce and distribute publicly paper and electronic copies of this thesis document in whole or in part.

Author

Joint Program in Physical Oceanography Massachusetts Institute of Technology Woods Hole Oceanographic Institution May 20, 2011

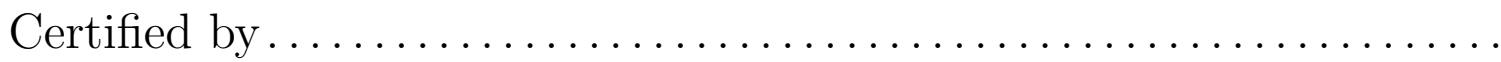

Paola Malanotte-Rizzoli

Professor

Thesis Supervisor

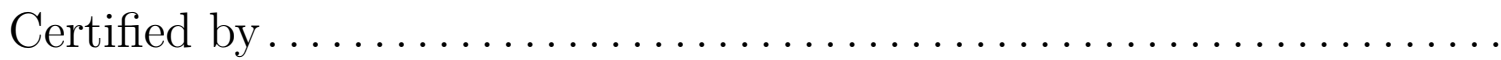

Michael A. Spall

Senior Scientist

Thesis Supervisor

Accepted by

Karl R. Helfrich

Chair, Joint Committee for Physical Oceanography 
TO MY PARENTS

献给我的父亲母亲 


\title{
Instabilities of an Eastern Boundary Current with and without Large-scale Flow Influence
}

by

Jinbo Wang

\author{
Submitted to the Joint Program in Physical Oceanography - Massachusetts \\ Institute of Technology / Woods Hole Oceanographic Institution \\ on May 20, 2011, in partial fulfillment of the \\ requirements for the degree of \\ Doctor of Philosophy in Physical Oceanography
}

\begin{abstract}
Eastern oceanic boundary currents are subject to hydrodynamic instability, generate small scale features that are visible in satellite images and may radiate westward into the interior, where they can be modified by the large-scale circulations. This thesis studies the stability of an eastern boundary current with and without the large-scale flow influence in an idealized framework represented by barotropic quasi-geostrophic dynamics.

The linear stability analysis of a meridional current with a continuous velocity profile shows that meridional eastern and western boundary currents support a limited number of radiating modes with long meridional and zonal wavelengths and small growth rates. However, the linearly stable, long radiating modes of an eastern boundary current can become nonlinearly unstable by resonating with short trapped unstable modes. This phenomenon is clearly demonstrated in the weakly nonlinear simulations. Results suggest that linearly stable longwave modes deserve more attention when the radiating instability of a meridional boundary current is considered.

A large-scale flow affects the short trapped unstable mode and long radiating mode through different mechanisms. The large-scale flow modifies the structure of the boundary current to stabilize or destabilize the unstable modes, leading to a meridionally localized maximum in the perturbation kinetic energy field. The shortwave mode is accelerated or decelerated by the meridional velocity adjustment of the large-scale flow to have an elongated or a squeezed meridional structure, which is confirmed both in a linear WKB analysis and in nonlinear simulations. The squeezed or elongated unstable mode detunes the nonlinear resonance with the longwave modes, which then become less energetic. These two modes show different meridional structures in kinetic energy field because of the different mechanisms.

In spite of the model simplicity, these results can potentially explain the formation of the zonal jets observed in altimeter data, and indicate the influence of the large-
\end{abstract}


scale wind-driven circulation on eastern boundary upwelling systems in the real ocean. Studies with more realistic configurations remain future challenges.

Thesis Supervisor: Paola Malanotte-Rizzoli

Title: Professor

Thesis Supervisor: Michael A. Spall

Title: Senior Scientist 


\section{Acknowledgments}

I owe a debt of gratitude to my advisors, Paola Rizzoli and Mike Spall, and my thesis committee, Glenn Flierl, Ken Brink, and Markus Jochum. I thank Paola for her constant support, care, trust, supervising for all these years; thank Mike for providing me the constructive comments that always drew my attention away from pure numerics to related physical questions and his research methodologies; thank Glenn for teaching me instability theory, giving me his models to start with, answering my numerous questions, and pointing me to the right direction; thank Ken for many helpful discussions on coastal dynamics, and his always encouraging conversations; and thank Markus for hosting my visits at NCAR during my first two years, and his sincere career advices. I benefited tremendously from numerous conversations with them. Their comments helped me improve this thesis. They not only taught me science, but more importantly, the way of doing science.

I am very grateful to Joe Pedlosky for many inspiring conversations, his friendship, reading and commenting on my thesis draft, and chairing my thesis defense. I never walked out of his office without having learned something new. I owe thanks to Steven Lentz and Fiamma Straneo for their emotional support during my early study.

I thank Hristina Hristova for the helpful discussions during the early stage of this thesis. I also thank Jean-Michel Campin, Maxim Nikurasin, and John Taylor for the helpful conversations on numerical methods; and Raf Ferrari, Carl Wunsch, Ian Fenty, and Da Zhu for their comments and suggestions.

I am indebted to Ruixin Huang for introducing me to Physical Oceanography and the MIT/WHOI Joint Program, and for his care and inspiration. I would not be here without him.

I am grateful to my friends in PO, PAOC, and JP/EAPS communities, especially my classmates Evgeny Logvinov, Christie Wood, Peter Sugimura, Martha Buckley, Andrew Barton, and my officemates Ian Fenty and Kjetil Våge for their invaluable comradeship and friendship. You made my life here much more enjoyable. I am also 
grateful to former PO students Dave Sutherland, Melanie Fewings, Greg Gerbi, and Yohai Kaspi for their help and advice during the early years.

Most importantly, I thank my wife, Xiaoyan, who has always been the source of my strength and happiness.

This work was supported by the NOAA project, NA05OAR4311132, and the MIT anonymous Y-S Fellowship. 


\section{Contents}

1 Introduction $\quad 11$

1.1 Eastern boundary currents and large-scale circulations . . . . . . . . 12

1.2 Theoretical background . . . . . . . . . . . . . . . . . . . . 19

1.3 Thesis outline . . . . . . . . . . . . . . . . . . 21

2 Barotropic instability of a meridional jet 23

2.1 Introduction . . . . . . . . . . . . . . . . . . 23

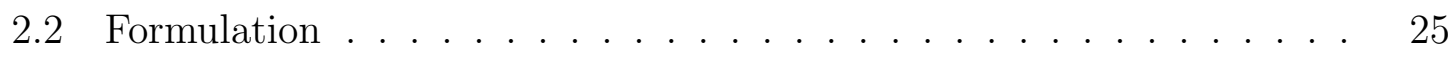

2.2.1 Equations .................... . . 25

2.2.2 Review of the stability of a Bickley jet . . . . . . . . . . 27

2.2.3 Methodology . . . . . . . . . . . . . . . . . . . . . . 28

2.3 Results . . . . . . . . . . . . . . . . . . . . . 31

2.3.1 The influence of a side boundary on the stability of a meridional Bickley jet . . . . . . . . . . . . . . 31

2.3.2 The influence of $\beta$ on an isolated meridional Bickley jet . . . . 33

2.3.3 The $\beta$ effect on meridional boundary currents . . . . . . . . . 52

2.4 Conclusion and discussion . . . . . . . . . . . . . . . . 64

3 Instability of an eastern boundary current: a nonlinear study $\quad 67$

3.1 Introduction . . . . . . . . . . . . . . . . . . 68

3.2 Methodology . . . . . . . . . . . . . . . . . . . . . 69

3.2 .1 Model ....................... 69 


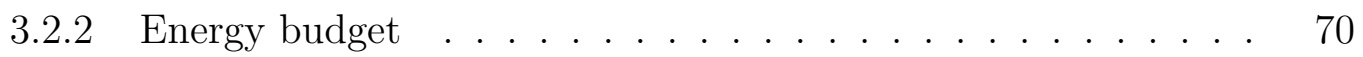

3.2 .3 Forcing . . . . . . . . . . . . . . . . . . . . 72

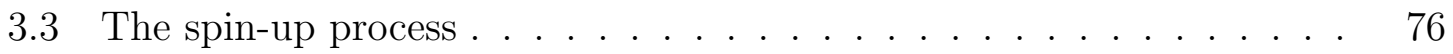

3.4 Mechanisms . . . . . . . . . . . . . . . . . . . . . . 88

3.4 .1 Linear growth rates . . . . . . . . . . . . . . . . . 91

$3.4 .2 \quad$ Case $\omega_{2}=2 \omega_{1} \ldots \ldots \ldots \ldots \ldots \ldots$. . . . . . . . . 92

$3.4 .3 \quad$ Case $\omega_{2} \neq 2 \omega_{1} \ldots \ldots \ldots \ldots \ldots \ldots \ldots \ldots$

3.5 Conclusion and discussion . . . . . . . . . . . . . . . . 106

\section{Instability of an eastern boundary current influenced by a large-scale} flow: a linear study $\quad 111$

4.1 Introduction . . . . . . . . . . . . . . . . . . . . . . . 111

4.2 Background review . . . . . . . . . . . . . . . . . . . . . 113

4.2 .1 Temporal and spatial instability . . . . . . . . . . . . 113

4.2 .2 Local and global mode . . . . . . . . . . . . . . . . . 116

4.2.3 Absolute and convective instability . . . . . . . . . . . 116

4.3 Formulation . . . . . . . . . . . . . . . . . . . . . 118

4.3.1 Large scale flow, a partial-gyre . . . . . . . . . . . . . . . 119

4.3.2 Large scale flow, a double-gyre . . . . . . . . . . . . . . . 122

4.4 WKB solution for the partial-gyre flow . . . . . . . . . . . 123

4.4 .1 Formulation . . . . . . . . . . . . . . . . . . 123

4.4 .2 Results . . . . . . . . . . . . . . . . . . . . 126

4.4 .3 Summary . . . . . . . . . . . . . . . . . . . . . 134

4.5 Numerical simulations . . . . . . . . . . . . . . . . . . . . 136

4.5.1 Spatial instability of a boundary jet with a localized forcing . 136

4.5.2 Pulse instability of a parallel boundary jet . . . . . . . . . 141

4.5.3 Pulse instability of a boundary jet modified by a partial-gyre flow 143

4.5.4 Pulse instability of a boundary jet modified by a double-gyre flow 152

4.6 Conclusion and discussion . . . . . . . . . . . . . . . . 166 
5 Instability of an eastern boundary current influenced by a large-scale flow: a nonlinear study 171

5.1 Introduction . . . . . . . . . . . . . . . . . . . . 172

5.2 Model ............................... 172

5.2.1 The vorticity equation . . . . . . . . . . . . . 172

5.2 .2 Forcing field . . . . . . . . . . . . . . . . . . . . . 172

5.3 Experiments . . . . . . . . . . . . . . . . 175

5.4 Results . . . . . . . . . . . . . . . . . . . . 177

5.5 Mechanisms ........................ 183

5.5.1 Phase- PII . . . . . . . . . . . . . . 183

5.5.2 Phase - PIII . . . . . . . . . . . . . . . . 191

5.6 Conclusion and discussion . . . . . . . . . . . . . . . 196

6 Conclusion 201

6.1 Instability of an eastern boundary current . . . . . . . . . . . . 201

6.2 The influences of large-scale flows . . . . . . . . . . . . . 206

6.3 Discussion . . . . . . . . . . . . . . . . . . . . . . 209

$\begin{array}{ll}\text { A Numerical model } & 213\end{array}$

$\begin{array}{ll}\text { B Eigenvalue problem } & 215\end{array}$

$\begin{array}{ll}\text { C Matrix perturbation } & 217\end{array}$ 
THIS PAGE INTENTIONALLY LEFT BLANK 


\section{Chapter 1}

\section{Introduction}

The term "Eastern Boundary Current" (EBC) can be used in different scenarios. In this thesis, it refers to the currents flowing along the west coast of major continents at low or mid-latitudes.

Narrow EBCs in the ocean are usually hydrodynamically unstable (Barth, 1989a, 1994; McCreary et al., 1991; Marchesiello et al., 2003), generating instabilities characterized by filaments, squirts, and eddies that are visible in satellite images (Ikeda and Emery, 1984; Brink, 1987; Flament et al., 1985; Narimousa and Maxworthy, 1989; Ikeda and Emery, 1984; Davis, 1985a; Flament et al., 1985; Brink, 1987; Barton et al., 1998; Marchesiello et al., 2003). These mesoscale features play crucial roles in exchanging water properties, such as temperature, salinity, nutrients, and sediments, between the shelf and the open ocean. They are also likely to radiate westward, becoming energy sources for the ocean interior (Hristova et al., 2008). However, the mechanisms of the radiation are not well studied, and the significance of its influence on the ocean interior is unknown.

EBCs are not isolated systems. They are open to large-scale circulations and subject to their influences. The large-scale circulation can transport interior water masses into EBC regions to change the local water properties (Freeland et al., 2003). It can also modify radiation from the EBC into the interior by affecting the Rossby wave propagation (Pedlosky, 1987). However, the instability radiation of an EBC is 
not well understood, and the interaction between an EBC and a large-scale circulation has not been investigated.

In this thesis we apply an idealized model to an EBC and a large-scale circulation to study the radiating instabilities of the EBC with and without the large-scale flow influence. In the following, we introduce the problem by reviewing the features of several major EBC systems from an observational point of view, and then formulate the problem, building on previous studies.

\subsection{Eastern boundary currents and large-scale cir- culations}

Four major EBC systems are reviewed here. They are the California current system, the Canary upwelling region, the Benguela current along the Southwest African coast, and the Leeuwin current in the Indian ocean along the west coast of Australia. No extensive review is intended, but the dominant features are described and their similarities and discrepancies are summarized. This helps to motivate the idealized approach used in the thesis.

\section{The California Current system}

The California Current system is the most extensively studied EBC region. It is also one of the major coastal upwelling regions. It is considered to consist of several currents. In the mid-latitude, a broad California Current extending about $1000 \mathrm{~km}$ offshore flows slowly equatorward; a narrower and weaker California Undercurrent flows poleward in the subsurface layer; and the weaker Davidson Current flows poleward at the surface near the coast (Batteen, 1997).

Most observations are conducted over the shelf region where coastal upwelling is prominent in response to the strong alongshore wind. Mesoscale features are ubiquitous over the coastal upwelling region, and they can be seen in satellite images and 
surface drifter data (Bernstein et al., 1977; Davis, 1985a,b; Kelly, 1985; Kosro and Huyer, 1986; Strub et al., 1991, 1995). Figure 1-1 is an example of a satellite infrared image showing mesoscale structures. The light-gray color indicates lower surface temperatures due to upwelling. The T-shaped filaments are surface expressions of dipole eddy pairs, which diverge after drawing the cold water seaward. These cold filaments

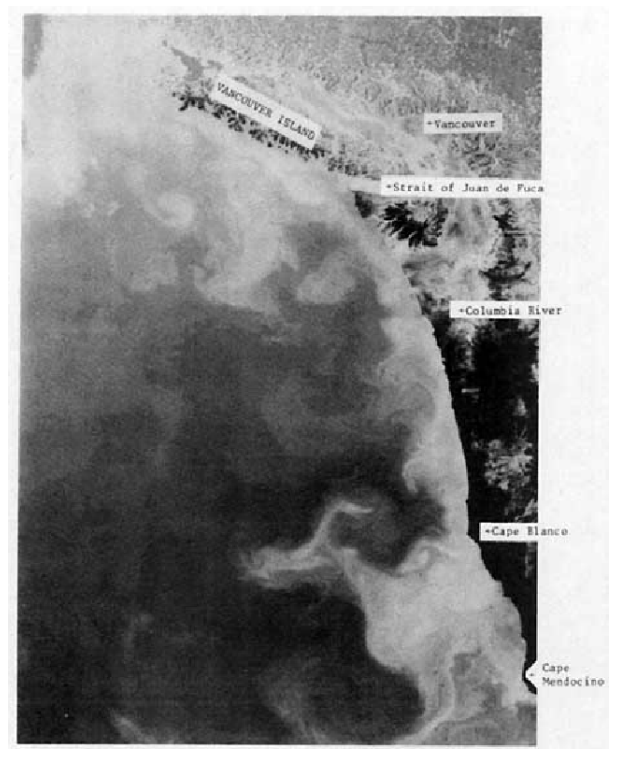

Figure 1-1: Satellite infrared image taken by Tiros-N on 2 October 1980 over northern California coast. The light color indicates lower surface temperatures. (Ikeda and Emery, 1984)

are frequently observed in many EBC regions. They are typically less than $100 \mathrm{~km}$ wide but extend hundreds of kilometers offshore from the coast (Brink and Cowles, 1991). In the vertical, they extend from the surface to depths of over $200 \mathrm{~m}$ and separate fresher, warmer, chlorophyll-depleted water from colder, saltier, chlorophyll-rich water (Huyer et al., 1991; Strub et al., 1991).

The mesoscale features are connected to a broader boundary region after streaming further offshore. The meanders and eddies associated with the coastal jet contribute to the eddy kinetic energy and water masses of the nearby deep ocean (Kelly et al., 1998; Strub and James, 2000). The speed of the westward movement of the eddy field is consistent with Rossby wave dynamics, which is then proposed to be the underlying 
dynamical mechanism, even though it has not yet been fully demonstrated. Besides the westward movement of the mesoscale features, the equatorward jet that develops next to the coast also has seasonal offshore variations, which can not be explained by the wind stress curl (Kelly et al., 1998).

On the basin scale, the California Current is considered to be the eastern limb of the North Pacific Current (the West Wind Drift). Figure 1-2 is the climatological dynamic height relative to $500 \mathrm{~m}$ in the Northeast Pacific taken from Strub and James (2002). The North Pacific Current between $20^{\circ} \mathrm{N}$ and $50^{\circ} \mathrm{N}$ flows eastward and diverges into the counterclockwise Alaska Gyre and the equatorward California Current.

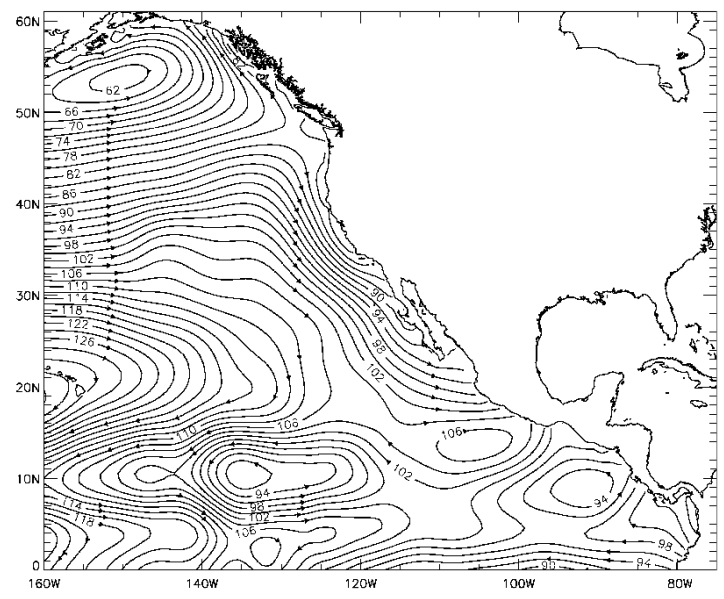

Figure 1-2: The climatological dynamic height relative to $500 \mathrm{~m}$ in the Northeast Pacific based on the temperature and salinity climatology of Levitus and Gelfeld (1992). (Strub and James, 2002)

The large-scale North Pacific Current can affect the EBC system by a direct mass contribution. It is observed that a cold anomaly extending from Vancouver Island to southern California is accompanied by an enhanced eastward flow in the North Pacific Current (Freeland et al., 2003). Figure 1-2 clearly shows the connection between the near-shore boundary current and the broader large-scale limb of the wind-driven gyre. 


\section{The Canary upwelling region}

The Northwest African coast, the Canary coast, is another well-known upwelling region with an EBC. The northeast trade winds along the African coast produce a surface equatorward Canary Current and a subsequent upwelling (Mittelstaedt, 1991). Meanders and filaments often develop in the Canary Current and extend $100 \mathrm{~km}$ offshore. To the west of the coastal current, there is the eastern limb of the North Atlantic Subtropical gyre flowing eastward. It is not dynamically understood how the large-scale circulation interacts with the Canary Current.

Another important phenomenon is the Cape Verde frontal zone residing in a largescale confluent flow (Zenk et al. 1991). It has been proposed that the instabilities in the frontal zone can excite radiating baroclinic Rossby waves and are a source of the mid-depth mesoscale features (Spall, 1992). The energy of these baroclinic waves propagates upstream (eastward) relative to the large-scale flow direction. However, it is not clear how these instabilities are modified by the large-scale confluent flow, and whether the confluent flow influences the offshore transport of the coastal mesoscale eddies.

\section{The Southwest African coast}

The coexistence of the mesoscale and large-scale circulation is also obvious over the Southwest African coast. The southward flowing warm Angola Current and the northward flowing Benguela Current converge at around $15^{\circ} \mathrm{S}$ to form the Angola/Benguela front. This front is a permanent feature at the sea surface, maintained throughout the year in a narrow latitudinal band between $14^{\circ} \mathrm{S}$ and $16^{\circ} \mathrm{S}$ (Meeuwis and Lutjeharms, 1990). Mesoscale structures have been observed as a feature of the front (Shannon et al., 1987).

South of the Anogla/Benguela front to the southern tip of Africa, Cape Agulhas, there is an upwelling region along the African coast. Upwelling occurs preferentially at certain locations corresponding to narrow parts of the continental shelf, and the 
most intense upwelling cell happens at Lüderitz $\left(27^{\circ} \mathrm{S}\right)$ (Lutjeharms and Meeuwis, 1987). Unlike most observed filaments that extend about $300 \mathrm{~km}$ offshore, the long filaments originating from the upwelling region at Lüderitz occasionally extend more than $1000 \mathrm{~km}$ offshore (Lutjeharms et al., 1991). The thermal infrared image in Figure 1-3 shows an example of the extremely long filament off Lüderitz. The wave-like pattern is a signature of the colder surface temperature, which persisted for about 10 days. The partial entrainment of filaments along the periphery of one or more Agulhas rings generated at the Agulhas current retroflection may create these long wave-like features. However, no study has been carried out to explain the dynamics governing the process.

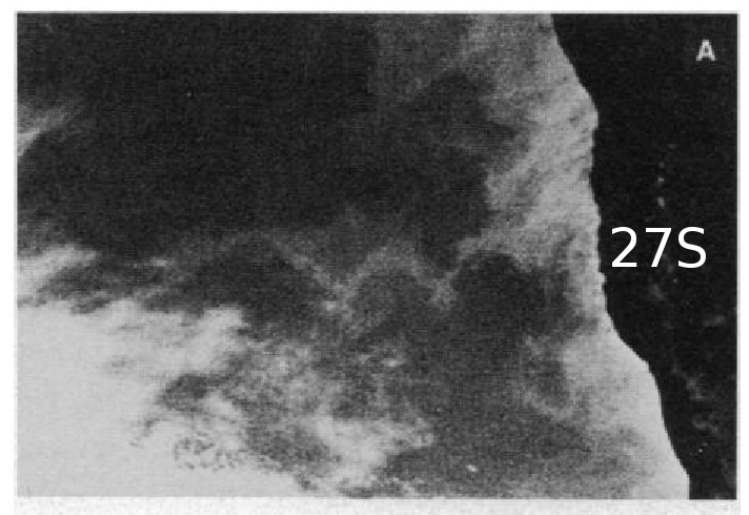

Figure 1-3: Thermal infrared image of the Southwest African coast from METEOSAT II taken on 5 July 1982 (Lutjeharms et al., 1991)

\section{The Leeuwin Current}

The importance of the large-scale circulation for a coastal current can be clearly seen in the boundary current along the west coast of Australia, the Leeuwin Current. The Leeuwin Current is an unusual EBC because it can not be related to local upwelling. The poleward surface current flows against the equatorward surface wind, and upwelling is absent although the surface wind is upwelling favorable (Cresswell 


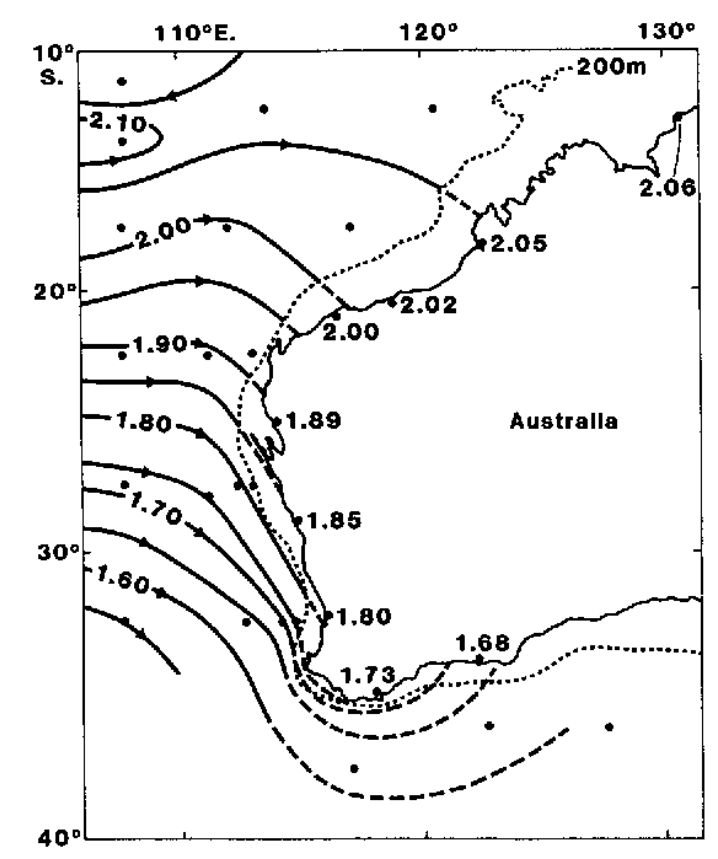

Figure 1-4: Contours of annual average steric sea level relative to $1300 \mathrm{db}$. The contours are linearly extrapolated into shore shown by the dashed contour-extensions. (Godfrey and Ridgway, 1985)

and Golding, 1980; Legeckis and Cresswell, 1981; Thompson, 1984).

Figure 1-4 shows the annual mean steric sea level relative to $1300 \mathrm{db}$. The broad interior geostrophic flow has an apparent eastward component. The interior flow impinges on the west coast of Australia to feed the strong southward Leeuwin Current.

It has been proposed that the along-shore pressure gradient is responsible for the generation of the Leeuwin Current. The along-shore pressure gradient can be produced by the Indonesian through-flow from the Pacific to the Indian ocean (Godfrey and Golding, 1981; Thompson, 1984; Godfrey and Ridgway, 1985) or by density forcing in the interior (McCreary et al., 1986; Batteen and Rutherford, 1990; Spall, 2003).

The Leeuwin Current is narrow $(<100 \mathrm{~km}$ wide), and swift $(>1 \mathrm{~m} / \mathrm{s})$. It carries warmer and fresher water from North West Cape poleward to Cape Leeuwin (Weaver and Middleton, 1989; Ridgway and Condie, 2004). Flowing poleward, the current loses heat to the atmosphere and to the interior. The heat loss to the interior is carried 
out by mesoscale eddies, which are generated by the boundary current instability (Batteen and Rutherford, 1990). Domingues et al. (2006) analyze a high resolution numerical simulation and show that eddy heat fluxes account for $70 \%$ of the heat loss of the boundary current. The eddy heat flux warms the ocean interior and cools the Leeuwin Current.

The connection between the Leeuwin Current and the adjacent large-scale onshore flow in the Indian ocean clearly demonstrates the importance of the large-scale circulation for the boundary current. How the large-scale flow changes the boundary current instabilities remains, however, unanswered.

\section{Discussion}

These major EBCs share similarities, but also exhibit differences. They are all adjacent to large-scale circulations in the open ocean and exhibit mesoscale features near the coast. However, long filaments, which extend more than $1000 \mathrm{~km}$ offshore, are occasionally observed in the Angola/Benguela front, but not in other EBCs. The upwelling filaments off Chile, which are not reviewed here, are qualitatively similar to those off California, but do not extend as far offshore, and are probably shorter-lived (Strub et al., 1995). These differences can not be explained by the local forcing only.

The characteristics of the large-scale flow also varies. A large circulation may extend thousands of kilometers like the subtropical gyres, or be generated by large rings, like Agulhas rings, which are of only hundreds of kilometers in scale. However, they have the same confluent or diffluent type of structure. These confluent and diffluent flows are the most common feature of the large-scale circulations, and may play significant roles in connecting the ocean interior with the boundary currents.

Because these EBC systems share many common traits, and because very little is known about how large-scale interior flow interact with EBCs, we propose an idealized simple model for these complex configurations. The model consists of a north-south oriented EBC and a large-scale flow with confluent and diffluent structures. The structure of the EBC and the large-scale flows are discussed in the following chapters. 


\subsection{Theoretical background}

Many studies have made use of local stability analyses to explain the generation of coastal mesoscale features. The mechanisms are essentially based on the local hydrodynamic instabilities of a boundary current (Griffiths and Linden, 1981; Ikeda and Emery, 1984; Barth, 1989a,b; Haidvogel et al., 1991; Allen et al., 1991; McCreary et al., 1991). Our interest here is to study the mechanism related to the radiating instabilities and the influence of the large-scale circulation. Previous studies demonstrated that zonality plays a crucial role for both the jet and the radiating instabilities. The north-south oriented boundary current, which is used as an idealization of the $\mathrm{EBC}$, behaves very differently from its zonal counterpart.

The energetic eddies in the ocean interior have motivated a series of studies concerning the origin of the eddy energy. They can be generated by weak meridional baroclinic gyres (Spall, 2000), or radiated from swift oceanic currents, such as the Gulf Stream. Many studies represent the Gulf Stream as a steady propagating northern boundary (Flierl and Kamenkovich, 1975; Pedlosky, 1977; Harrison and Robinson, 1979; Malanotte-Rizzoli et al., 1987). Their results provide important mechanisms governing the energy radiation from strong ocean currents. Talley (1983) derives the wave properties by solving for the stability of a steady zonal flow, and shows that the instability radiation will not occur unless there is a westward component in the zonal current or the far field is made baroclinic. The main argument is that the wave characteristics of the eastward-traveling instabilities do not match the dispersion relation of the free Rossby waves in the far field.

Instability radiation occurs more easily for a non-zonal current. Fantini and Tung (1987) study a meridional western boundary current and show that the instabilities generated by the meridional boundary current are able to radiate eastward even in the presence of realistic dissipation. Pedlosky (1993) investigates a baroclinic shear which is inclined with respect to a latitude circle. Although this study focuses on the generation of a boundary current by the boundary-trapping of two reflected unstable 
waves, it clearly demonstrates that a non-zonal flow is less stable and its instabilities can reach out to the far region. Kamenkovich and Pedlosky (1996, 1998a,b) explicitly studied the influence of nonzonality on the jet instability and the instability radiation. They found that even a slight nonzonality in the mean flow can generate radiating instabilities, which can significantly penetrate into the far field.

Following that thread, Hristova et al. (2008) studied the radiating instabilities of meridional boundary currents and compared a Western Boundary Current (WBC) with an EBC. They showed that an EBC supports a greater number of radiating modes over a wider range of meridional wavenumbers than a WBC. They use a piecewise constant meridional velocity profile as used in Fantini and Tung (1987) to represent the boundary current. The stability equation for a piecewise constant velocity profile is reduced to an ordinary differential equation with constant coefficients, which is easier to solve. But it can not give a realistic shortwave cutoff. Furthermore, the piecewise profile is difficult to implement in numerical simulations. We extend these previous studies by representing the boundary current with a continuous velocity profile and focus on the stability of an EBC on a beta plane. Both linear and nonlinear regimes are considered.

The radiating instabilities of an EBC, as indicated in Hristova et al. (2008), have large zonal wavelengths and small westward decay rates, which result in zonal jet structures in the interior. They argued that these zonal structures resemble the zonal jets observed from the altimeter data by Maximenko et al. (2005). The observed mid-latitude zonal jets have a meridional wavelength of about $300 \mathrm{~km}$ and a rootmean-square velocity of $6.9 \mathrm{~cm} / \mathrm{s}$. It will be discussed in the nonlinear simulations considered in this thesis whether radiating instabilities of an EBC can generate similar features.

The stability problem becomes more complex once a large-scale circulation is included. On the one hand, waves can be affected by the large-scale circulation in the interior by the Doppler effect (Pedlosky, 1987; Liu, 1999). On the other hand, the boundary current can be influenced by the onshore/offshore large-scale flows. The 
boundary current is modified by the large-scale flow and becomes non-uniform in the streamwise direction. The related theories are discussed in the following chapters.

\subsection{Thesis outline}

As few previous studies considered a systematic development of the instability theory of meridional currents with a continuous velocity profile in both the linear and nonlinear regimes, we consider a barotropic quasi-geostrophic model as the simplest starting point. This simplicity helps to focus on a limited number of dynamical factors and to identify mechanisms that can be used for future studies. The model is described in Chapter 2, and is used throughout this thesis.

The linear stability analysis of a meridional current with a continuous velocity profile is studied over a wide range of parameter space in Chapter 2. It compares the instability of a zonal current with that of a meridional current on a beta plane; investigates the effect of a solid boundary on an $f$ plane current, and the effect of beta on parallel meridional currents. The radiating instabilities of meridional boundary currents on a beta plane are also studied.

Chapter 3 extends the linear study of an EBC to a weakly nonlinear regime. Linearly stable radiating modes can become unstable because of the nonlinear energy transfer. They have small zonal wavenumbers and show narrow zonal jet structures in the interior. The radiating instabilities from the EBC can be a possible source for the observed interior zonal jets.

In Chapters 4 and 5, an idealized large-scale circulation is added, and its influence on the boundary current stability is studied both in the linear regime (Chapter 4), and in the nonlinear regime (Chapter 5). The linear analyses provide a useful guide in explaining the nonlinear results. 
THIS PAGE INTENTIONALLY LEFT BLANK 


\section{Chapter 2}

\section{Barotropic instability of a meridional jet}

\section{$2.1 \quad$ Introduction}

Unstable currents are one of the main sources of mesoscale activities in the atmosphere and ocean. The instabilities of the unstable currents may be trapped locally around the unstable region or radiate away to influence the far field. Previous studies show that non-zonal flows are less stable and more likely support radiating instability than zonal ones (Kamenkovich and Pedlosky, 1996; Spall, 2000; Walker and Pedlosky, 2002). Oceanic flows in the mid-latitude rarely coincide with latitude circles because of the presence of continental boundaries, so that the non-zonality becomes one of the important characteristics of those flows. Stability analysis has been carried out mostly for zonal flows, both on the $f$ and beta planes. Meridional flow instabilities, however, are less studied. In this chapter, we revisit the linear stability problem of a meridional current, which sets the foundation for discussions in following chapters.

The energetic nature of the disturbances is often attributed to instabilities of the mean currents. Depending on the energy source, instabilities can be categorized as barotropic or baroclinic. In the barotropic case, the energy of disturbances comes from the kinetic energy of the mean flow. In the baroclinic case, the energy for disturbances 
comes from the available potential energy due to the vertical shear. Because of the mathematical complexity of the problem when the potential vorticity gradient has both meridional and zonal components, we will use a simple barotropic model with a flat bottom. More complex dynamics, such as the baroclinicity and sloping bottom, will be considered in future studies.

Barotropic instability theory dates back to the instability studies by Helmholtz and Reynolds (discussed in Lamb 1932). Rayleigh (1913) studied the stability of horizontal parallel flows and found that the shape of the velocity profile determines the stability of the current. Kuo (1949) extended Rayleigh's theory to geostrophic flow, for which the curvature of the earth surface, denoted as the beta effect, may become important. Applying an integral constraint, he found that in order to support the unstable wave perturbation, the zonal current, $U(y)$ must have such a structure that $\left(\beta-U_{y y}\right)$ changes sign inside the current. In other words, the cross-tream gradient of the absolute vorticity of the mean current changes sign somewhere in the flow. Kuo (1949) also found that a positive $\beta$ stabilizes the westerlies but destabilizes the easterlies. It is clear from the criterion $\left(\beta-U_{y y}\right)$ that $\beta$ introduces an asymmetry as $U$ changes sign. On the other hand, if $\beta$ is negative, the stability of the westerly becomes the same as the stability of the easterly with positive $\beta$. Howard and Drazin (1964) showed that changing the sign of $\beta$ is equivalent to reversing the zonal flow. Barotropic instability for zonal flows is reviewed in Kuo (1973) and systematically formulated in Pedlosky (1987).

In the ocean, however, many currents have a dominant meridional component because of the presence of the continental boundaries. The meridional component of the current has a significant effect on the flow stability. Walker and Pedlosky (2002) studied the baroclinic instability of a meridional flow in a channel and found that no minimum shear is required for the flow to be unstable. Kamenkovich and Pedlosky (1996) studied the effect of the non-zonality on jet stability with a focus on the radiating instabilities. They found that the non-zonality makes flows less stable and more able to support radiating instabilities than their zonal counterparts. The 
effect of the meridional component of the flow can be singled out by considering a pure meridional current. Fantini and Tung (1987) studied the stability problem of a barotropic meridional western boundary current (WBC) using a piece-wise constant velocity profile, an idealization of the Gulf Stream. Hristova et al. (2008) extended the study of Fantini and Tung (1987) to include a comparison of the eastern boundary current $(\mathrm{EBC})$ with the $\mathrm{WBC}$ and found that the EBC supports more radiating modes than the WBC. EBCs are more likely to excite zonal jets in the oceanic interior because their radiating modes have large zonal wavelengths. We extend these previous studies by considering meridional jets with a continuous profile over a larger parameter space.

This chapter is organized in three sections. Section 2.2 discusses the model formulation with a brief review of results from previous studies. Section 2.3 presents results. Section 2.4 gives the conclusion and discussion.

\subsection{Formulation}

\subsubsection{Equations}

Because of the complexity of the problem, we start with the simplest model described by the barotropic quasi-geostrophic vorticity equation. Here jet refers to the uniform parallel flow, which is assumed to be steady and maintained by an external forcing. The term basic state denotes the initial current.

The barotropic quasi-geostrophic $(\mathrm{QG})$ potential vorticity equation is written as:

$$
\begin{aligned}
& \partial_{t} q+J(\psi, q)=\mathcal{F} \\
& q=\nabla^{2} \psi+\beta y
\end{aligned}
$$

where $\psi$ is the streamfunction, $q$ is the potential vorticity, $\mathcal{F}$ represents the forcing and dissipation, and $\beta$ represents the meridional variation of the Coriolis parameter. Following the conventional linear stability analysis, we assume the field can be de- 
composed into a basic state (denoted by overbar) and a perturbation (denoted by primes). Then the above equation can be linearized to

$$
\partial_{t} q^{\prime}+J\left(\bar{\psi}, q^{\prime}\right)+J\left(\psi^{\prime}, \bar{q}\right)=0
$$

where

$$
\begin{aligned}
\psi & =\bar{\psi}+\psi^{\prime} \\
q & =\bar{q}+q^{\prime}=\nabla^{2} \bar{\psi}+\beta y+\nabla^{2} \psi^{\prime} \\
\mathcal{F} & =J(\bar{\psi}, \bar{q}) .
\end{aligned}
$$

Using the width and the maximum velocity of the jet as scales, denoted as $L$ and $V$ respectively, the equation is nondimensionalized as

$$
\left(\partial_{t}+\bar{u} \partial_{y}+\bar{v} \partial_{x}\right) \nabla^{2} q^{\prime}+\left(\beta^{*}-\bar{u}_{y y}\right) \psi_{x}^{\prime}-\bar{v}_{x x} \psi_{y}^{\prime}=0
$$

where $\beta^{*}=\beta L^{2} / V$.

Equation 2.5 is further simplified to (after dropping the primes and asterisks without loss of generality)

$$
\left(\partial_{t}+\bar{u} \partial_{x}\right) \nabla^{2} \psi+\left(\beta-\bar{u}_{y y}\right) \psi_{x}=0
$$

for zonal flows, and to

$$
\left(\partial_{t}+\bar{v} \partial_{y}\right) \nabla^{2} \psi+\beta \psi_{x}-\bar{v}_{x x} \psi_{y}=0
$$

for meridional flows.

In this study, we use the velocity profile $\bar{v}=-\operatorname{sech}^{2}(x)$, the so-called Bickley jet (Bickley, 1937), to represent the mean meridional jet. We chose this profile because its basic stability characteristics had been studied for zonal flows on both the $f$ and 
beta planes. We verify our methodologies by comparing our results with previous ones and extend the linear stability of the Bickley jet by considering meridional flows on $f$ and beta planes. The next section briefly reviews previous results of the stability a Bickley jet.

\subsubsection{Review of the stability of a Bickley jet}

Here we follow Lipps (1962), Kuo (1973), and Maslowe (1991) to present a review of the stability of a zonal Bickley jet. No meridional case has been considered in the past.

A barotropic zonal Bickley jet is of the form $\bar{u}(y)=\operatorname{sech}^{2}(y)$. The perturbations can be represented by the normal mode solution in time and the streamwise direction with a $y$-dependent structure: $\psi=\phi(y) \exp (i k(x-c t))$. Equation 2.6 becomes

$$
(\bar{u}-c)\left(\partial_{y y}-k^{2}\right) \phi+\left(\beta-\bar{u}_{y y}\right) \phi=0 .
$$

The boundary conditions can be

$$
\phi=0 \text { at } y= \pm \infty
$$

for a non-radiation condition as used in Lipps (1962), or

$$
\phi_{x}-i k \phi=0 \text { at } y= \pm \infty
$$

for a radiation condition as used in Maslowe (1991). Because of the axisymmetric property of the jet, the problem can be simplified to include only half of the jet with two different boundary conditions

$$
\begin{aligned}
& \phi=0 \text { at } \quad y=0 \text { for the varicose mode } \\
& \phi^{\prime}=0 \text { at } \quad y=0 \text { for the sinuous mode }
\end{aligned}
$$


In the analytical study by Lipps (1962), the stability equation is transformed into Legendre's equation by a transformation of coordinate, $Z=\tanh y$. With Kuo's (1949) proof that $\beta-\bar{u}_{y y}=0$ at the critical point where $\bar{u}=c$, two main neutral solutions are found. One is the symmetric sinuous mode

$$
c=k^{2} / 6, \beta=k^{2}\left(4-k^{2}\right) / 6, \phi=\operatorname{sech}^{2} y
$$

and another is the antisymmetric varicose mode

$$
c=\left(3+k^{2}\right) / 6, \beta=\left(1-k^{2}\right)\left(3+k^{2}\right) / 6, \phi=\operatorname{sech} y \tanh y
$$

The neutral curves defined by real eigenvalues for those two modes are shown in Figure 2-1. The sign of $\beta$ indicates the direction of the zonal flow, $\beta>0$ represents the westerlies and $\beta<0$ represents the easterlies. According to the Rayleigh-Kuo stability condition for a sheared flow, the quantity $\beta-\bar{u}_{y y}$ must change sign somewhere in the shear flow for instabilities to occur. In this case, stability is assured for $\beta<-2$ because $\bar{u}_{y y}$ has a maximum value of 2 . The neutral solutions for a flow on an $f$ plane are $k=1$ for the sinuous mode (the red line), and $k=2$ for the varicose mode (the blue line).

There is an obvious asymmetry in the neutral curve between the eastward and westward flows. Easterlies are more unstable than the westerlies and support a larger range of unstable modes, which is also confirmed by Kuo (1973). This is an important asymmetry for the stability of a zonal jet on a $\beta$ plane. We will show later in this chapter that the stability of a meridional boundary jet also has an asymmetry, which is not introduced by the flow direction, but rather by the jet position (EBC or WBC).

\subsubsection{Methodology}

Two methods are used to solve the linear stability problem of a meridional Bickley jet. The linearized inviscid stability equation 2.7 can be simplified into an eigenvalue 


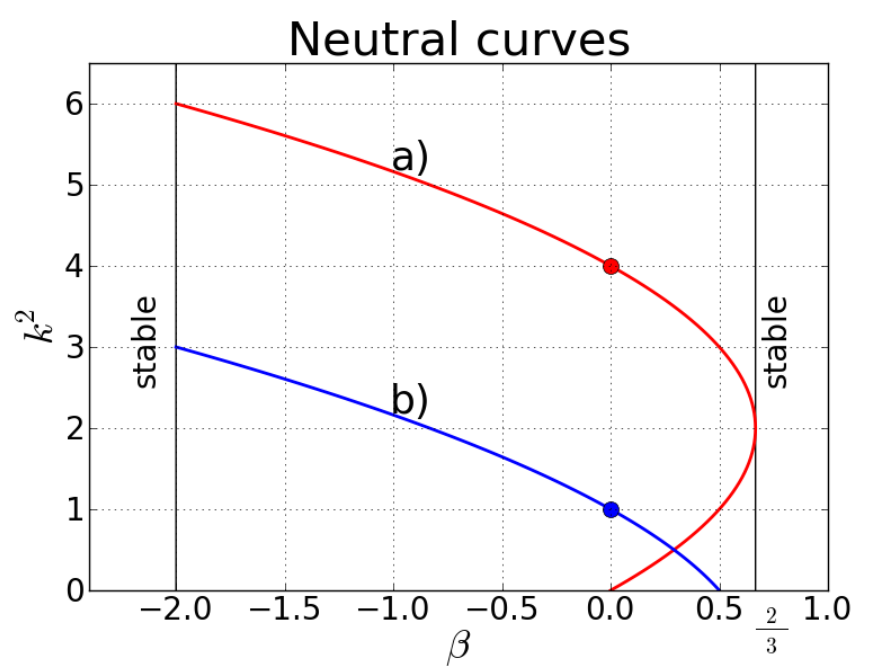

Figure 2-1: The neutral curves for a zonal jet $\bar{u}=\operatorname{sech}^{2} y$ reproduced following Maslowe (1991). (a) is for the sinuous mode, (b) is for the varicose mode. $k=1$ of the varicose mode and $k=2$ of the sinuous mode are the neutral wavenumbers for a Bickley jet on an $f$ plane $(\beta=0)$.

problem by substituting the normal mode solutions. The boundary conditions are given by equation 2.8. The eigenvalue problem is solved using either a finite difference method or a shooting method. Another way to solve equation 2.7 is to use a time dependent numerical model. More technical details of these methods are given in the appendix.

The left panel in Figure 2-2 shows the growth rate $l c_{i}$ calculated using the finite difference method. The $f$ plane case is considered here in order to compare with the results in Lipps (1962); Maslowe (1991). The stability boundaries reside at $l=1$ and $l=2$, which agree with the neutral solutions in Lipps (1962) (indicated by the two dots in Figure 2-1). The wavenumber $k$ is changed to $l$ to indicate that the jet is meridional. The right panel shows that the eigenfunctions of neutral modes at $l=1$ and $l=2$ also agree well with the analytical solutions in Lipps (1962).

The eigenvalue solutions are confirmed by a linear numerical calculation. The linear numerical model is initialized with a single Fourier mode with $l=0.6$ and is iterated from $t=0$ to $t=60$ (non-dimensional time scaled by $L / V$ ). Figure 2-3 shows that the streamfunction pattern from the linear numerical simulation (right) 
is almost identical to the eigenvalue solution (left).

Figure 2-4 shows the meridionally averaged kinetic energy at $x=0.16$. The match of the growth rate of the perturbation in the numerical calculation with that of the eigensolution validates both the eigenvalue method and the numerical model. The numerical method is described in Appendix A. The same codes are used in the following calculations.
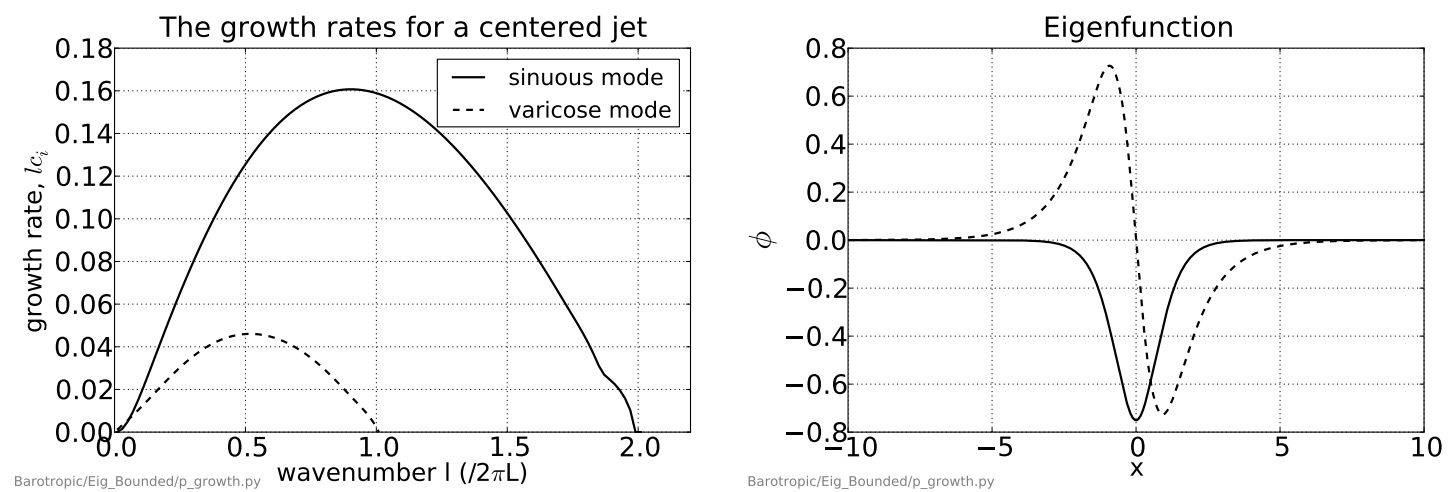

Figure 2-2: The left panel shows the growth rates as functions of the wavenumber. The structure of the eigenfunction for two neutral modes $l=1,2$ are shown in the right panel. The dashed line shows the neutral varicose mode $\phi=-\operatorname{sech} x \tanh x$, and the solid line shows the neutral sinuous mode $\phi=-\operatorname{sech}^{2} x$.
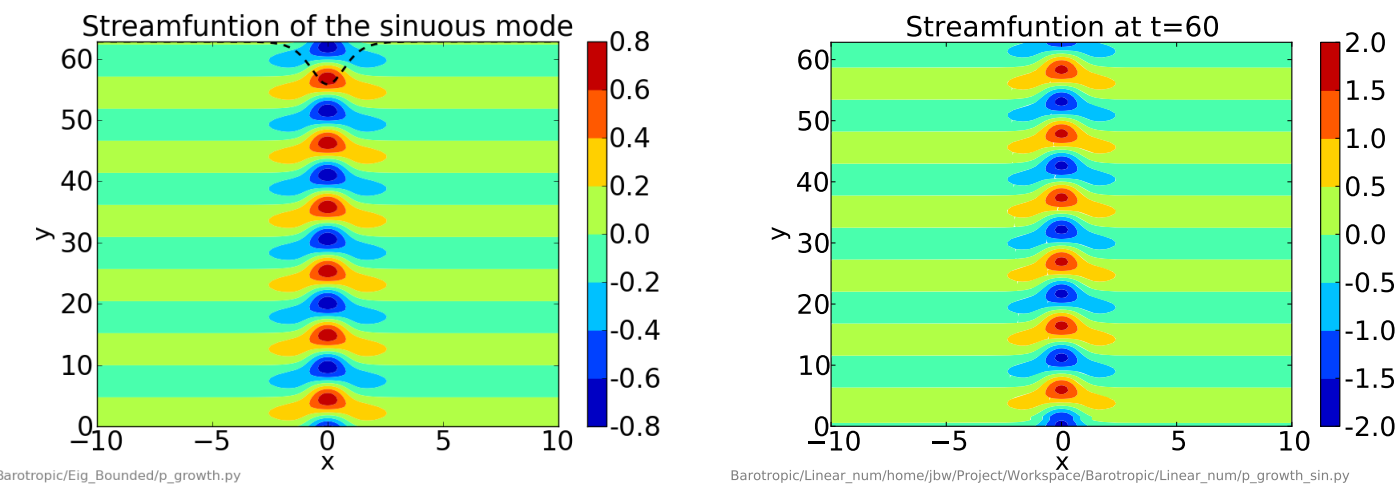

Figure 2-3: The perturbation streamfunction corresponding to wavenumber $l=0.6$. They are reconstructed from the eigensolution (left) and from the numerical simulation at $t=60$ (right). The perturbation in the numerical simulation is initialized using $q=\sin (0.6 y)$. The black dashed line in the left panel shows the structure of the meridional velocity of the centered Bickley jet. The perturbation streamfunction leans against the jet shear to extract energy from the basic state. 


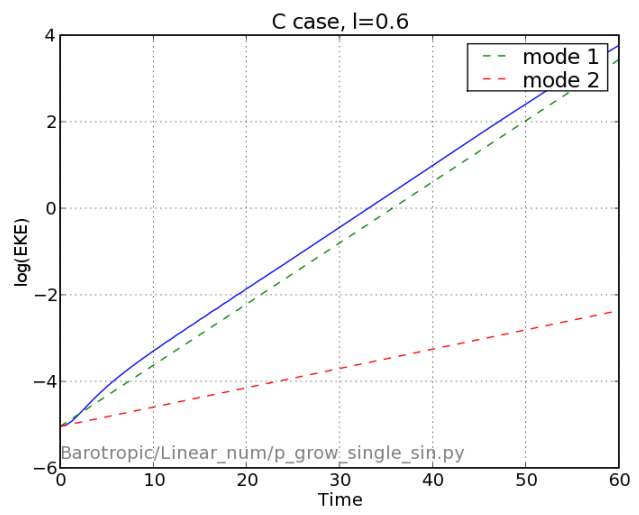

Figure 2-4: The kinetic energy, $E K E=\left(u^{2}+v^{2}\right) / 2$, in the numerical integration (the solid line) grows in time. Two dashed lines indicate the grow rates calculated in the eigenvalue problem. Two different methods, the direct numerical integration and the eigenvalue method show a consistent solution.

\subsection{Results}

\subsubsection{The influence of a side boundary on the stability of a meridional Bickley jet}

The impenetrable side wall boundary requires a no-normal-flow boundary condition, $u_{\perp}=0$. In the $\mathrm{QG}$ framework, this is equivalent to requiring $\psi$ to be constant along the boundary, $\psi=0$ without loss of generality. The side boundary effect is examined by varying the distance between the jet center and the boundary denoted by $d$ hereafter. The meridional current is represented by $\bar{v}=-\operatorname{sech}^{2}(x+d)$, where $x=-20 \cdots 0$. The calculation in this subsection is done on an $f$ plane with $\beta=0$. The boundary effects with a non-zero $\beta$ are considered in separate sections.

Figure 2-5 shows the growth rates for meridional Bickley jets with $d=10,2,1,0$. The growth rates for a centered jet $(d=10)$ is shown by red lines, which are identical to those in Figure 2-2 and are shown here as a reference. The growth rates decrease for both the sinuous mode (solid lines) and the varicose mode (dashed lines) as the jet moves towards the side boundary (from $d=10$ to $d=0$ ). However, the varicose modes show a different behavior from the sinuous modes. For sinuous modes (solid 


\section{the boundary effect on growth rates}

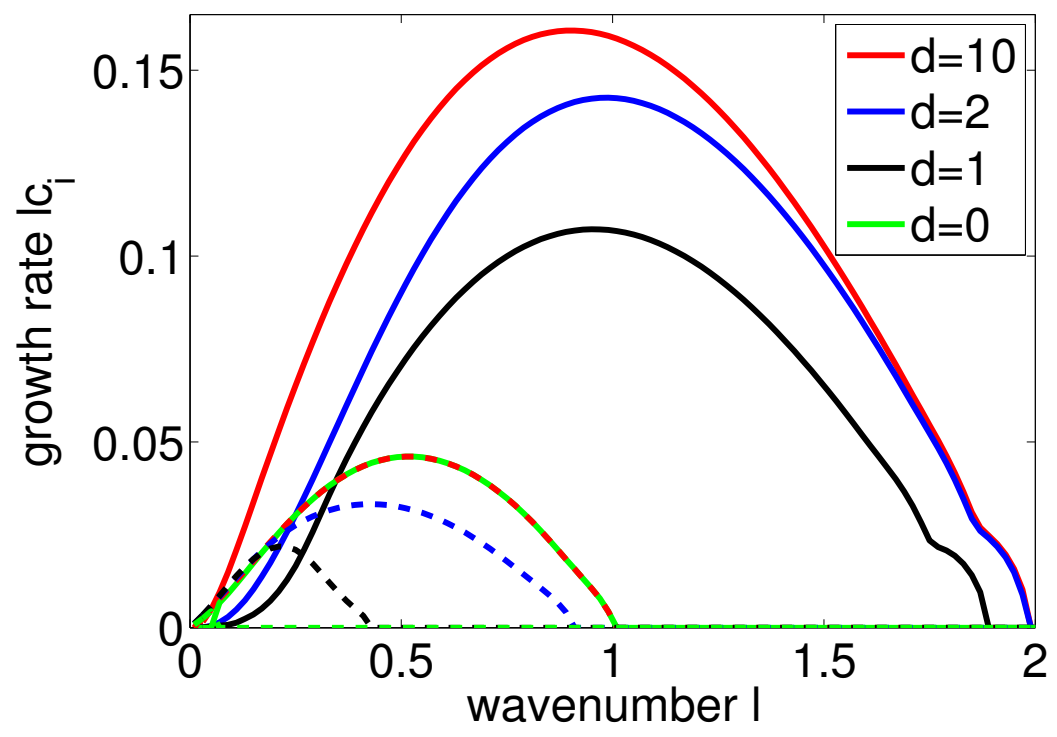

Figure 2-5: The growth rates for jets with different distances to the side boundary, $\bar{v}=-\operatorname{sech}^{2}(x-d)$ where $x=[-20 \cdots 0]$ and $d=[10,2,1,0]$, which are shown in the legend. The solid lines show sinuous modes and dashed lines varicose modes. The green line shows that when the jet is exactly cut in half by the boundary, the varicose mode is the only growing mode, and there is no sinuous mode as the boundary condition of the sinuous mode at the side boundary can not be satisfied.

lines), longer waves decay faster than the shorter waves, but for varicose modes, shorter waves are more affected by the side boundary. When the jet is exactly cut in half by the boundary (the $d=0$ case), the sinuous mode is eliminated because its boundary condition, $\partial_{x} \phi(x=0)=0$, can not be satisfied; while the growth rate curve of the varicose mode is unchanged because its boundary condition, $\phi(0)=0$, is naturally satisfied.

A numerical simulation shows the same result. Figure 2-6 shows the perturbation streamfunction at $t=60$ (left) and the time series of the EKE (right). The streamfunction is half of the varicose mode calculated in the case with a centered jet $(d=10)$. The EKE growth matches the growth rate from the eigensolution.

The suppressing effect of the boundary on the instability is shown in Figure 2-7. The boundary condition $(\phi=0)$ suppresses the mode that has a large crossstream 

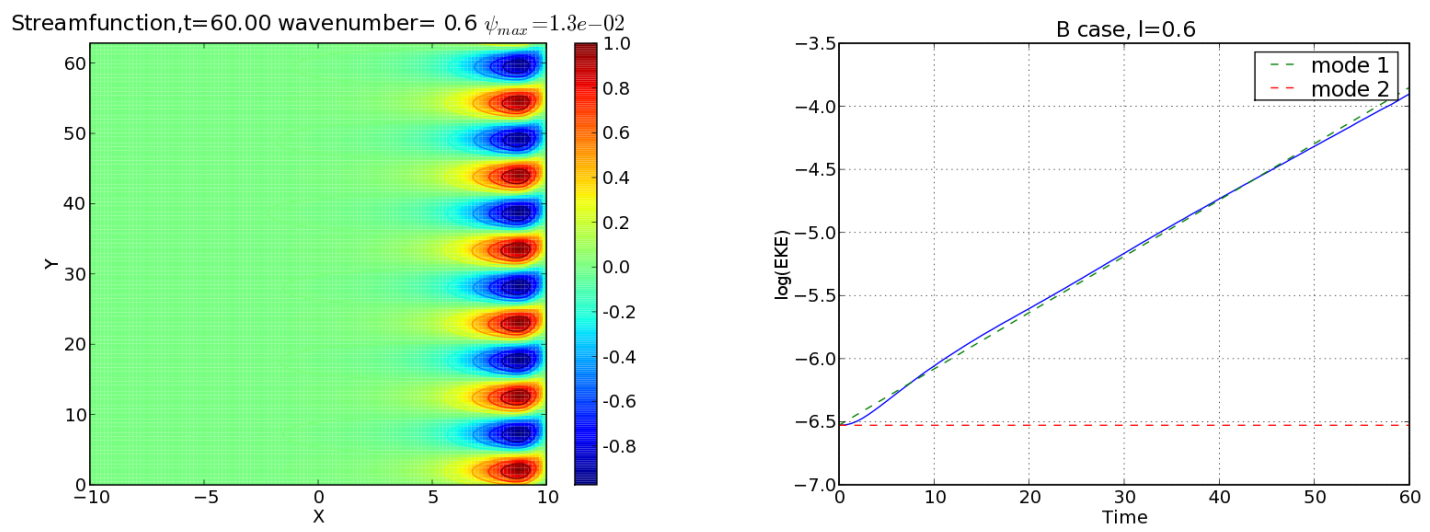

Figure 2-6: The streamfunction of the perturbation at $t=60$. The model is initialized with a perturbation field $q=\epsilon \sin (0.6 y)$. The right panel shows that the EKE grows with time. The growth rate matches the eigenvalue solution.

structure (upper and middle panels), and induces changes of the phase tilt in the perturbation field (lower panel). The Reynolds stress decreases (figure not shown), leading to a decrease of the overall growth.

To summarize, based on the calculation of the Bickley jet on an $f$ plane, it is shown that the boundary tends to suppress the growth of the unstable mode. When the Bickley jet is cut in half by the boundary, only the varicose mode grows. The varicose mode has been paid little attention in the previous literature as it has a smaller growth rate than the sinuous mode when the boundary effect is negligible. However, the varicose mode is the only mode if the boundary is placed in the middle of the jet. We will use a half Bickley jet with a side boundary to study the instability of a meridional boundary current in the later subsections.

\subsubsection{The influence of $\beta$ on an isolated meridional Bickley jet}

\section{On a bounded jet}

The eigenvalue problem formed from the stability equation can be solved by using the finite difference method with non-radiating boundary conditions. We here concentrate on the discussion of the growth rates and the spatial structure. The non-radiating 


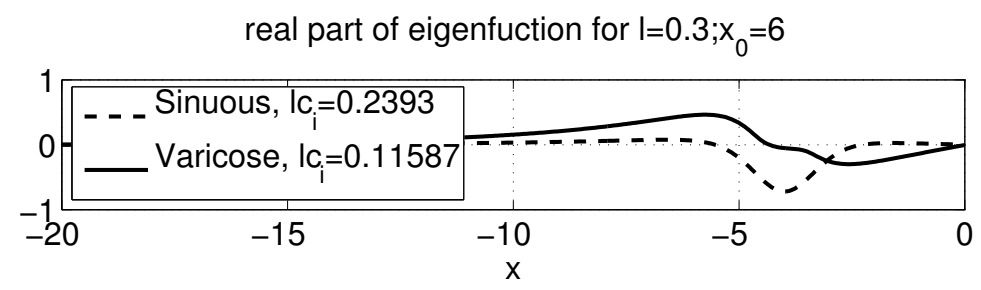

abs of the eigenfuction for $l=0.3$
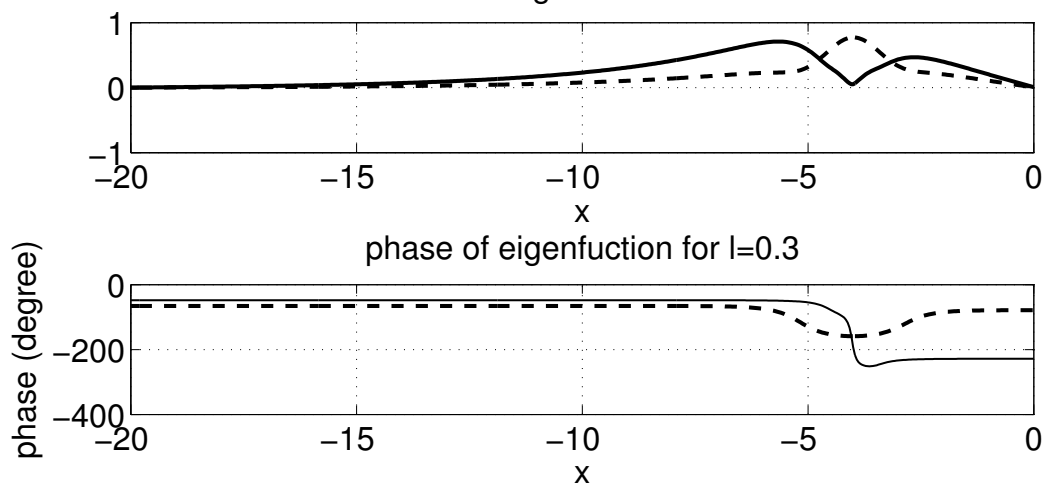

Figure 2-7: The zonal structure of the eigenfunction of the mode with the wavenumber $l=0.3$ for a jet profile with $d=4$. Dashed lines represent the modified-sinuous mode, and solid lines represent the modified-varicose mode. The upper panel shows the real part, the middle panel shows the absolute value, and the lower panel shows the phase. The phase modification is clear in the lower panel.

boundary conditions could be a problem for those radiating modes. In the next section, we solve the eigenvalue problem with radiating boundary conditions using a shooting method and discuss the radiating instability of the meridional Bickley jet.

The meridional Bickley jet on a $\beta$ plane has more types of unstable modes besides the dominating sinuous and varicose modes (Figure 2-8). As beta increases, the shortwave cut-off of the varicose mode (the thin lines) moves to higher wavenumber, and the longwave cut-off emerges. Overall, the beta effect reduces the growth rate (the red and blue lines are enveloped by the outer dashed line), but the influence on the varicose mode is different than the influence on the sinuous mode. Including $\beta$ increases the maximum growth rate of the varicose mode (the thin lines), but decreases the growth rate of the sinuous mode (the thick line with large growth rates).

The beta effect on the stability of a zonal jet is different from that of a meridional jet. In order to highlight the difference, we compute the growth rate of a zonal Bickley 


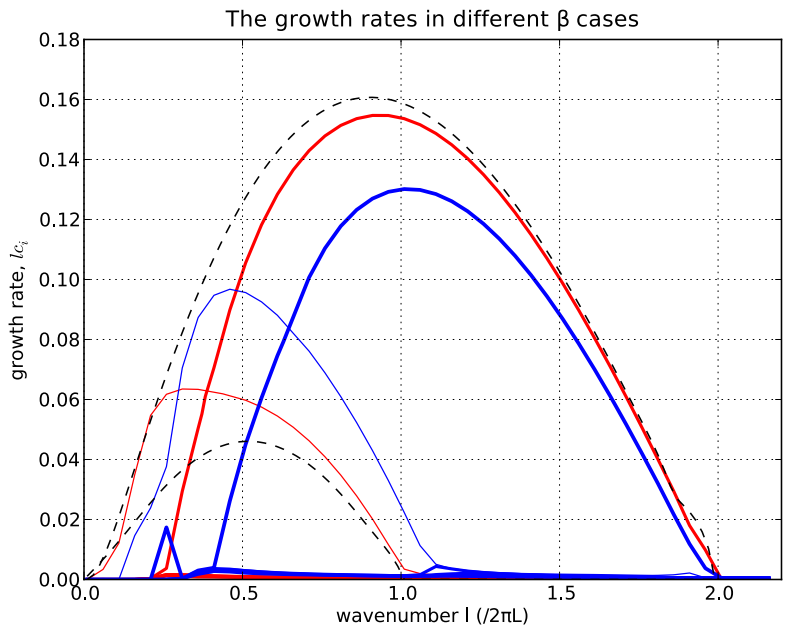

Figure 2-8: This figure shows the growth rates for a center jet in a beta plane with $\beta=0.1$ (red lines), $\beta=0.25$ (blue lines), and $\beta=0$ (dashed lines). The thick red and blue lines with large growth rates represent the modified sinuous mode, and the thin lines represent the modified varicose mode. Following the increase of $\beta$, the growth rates increase/decrease for the varicose/sinuous mode. The sinuous mode shows more dramatic decreases for longer waves than for shorter waves.

jet for various betas and compare them with those of the meridional jet. The results are shown in Figure 2-9.

For a zonal jet, there are only two types of modes, the varicose and sinuous modes. $\beta$ stabilizes both sinuous and varicose modes. As $\beta$ increases, a shortwave cutoff appears for the varicose mode, and a longwave cut-off appears for the sinuous mode.

The differences are clear by comparing the growth rates of a zonal and a meridional jet side by side. Beta stabilizes the sinuous mode for both the zonal and meridional jet, especially over the longwave end, while the stabilizing effect is weaker for the meridional jet than for the zonal jet. The weaker effect can be shown by comparing the discrepancies between the red and blue lines in the left panel with the discrepancies between the red and green lines in the right panel. $\beta$ stabilizes the varicose mode of the zonal jet, but destabilizes the varicose mode of the meridional jet. 

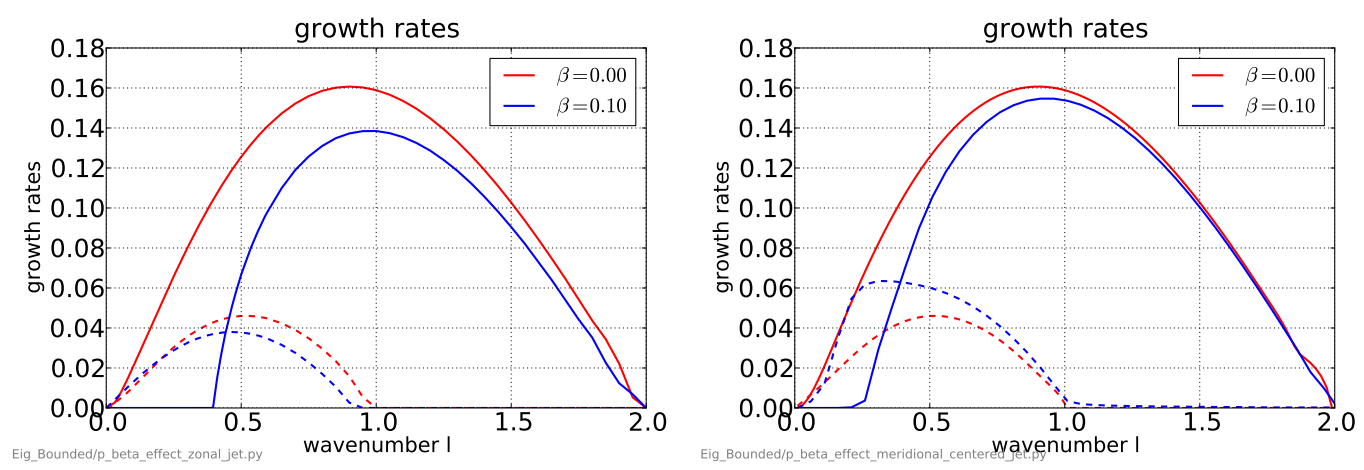

Figure 2-9: The left panel shows the growth rate as a function of wavenumber for a zonal Bickley jet. Increasing beta causes the growth rate to decrease for both the varicose mode (dashed lines) and the sinuous mode (solid lines). The right panel shows the growth rates for a meridional Bickley jet.

If the jet is purely meridional, equation (2.5) is

$$
\left(\partial_{t}+\bar{v} \partial_{y}\right) \nabla^{2} \psi+\beta \psi_{x}-\bar{v}_{x x} \psi_{y}=0
$$

then the vorticity gradient involves a zonal component $\bar{v}_{x x}$ in addition to the meridional component $\beta$. The conventional approach of finding the necessary condition for the instability using the integral method discussed by Kuo (1949) possibly fails (Walker and Pedlosky, 2002). Here we use a perturbation method to study the mechanism of the $\beta$ effect on the stability of the meridional jet. Assuming $\beta$ is a small parameter, $\beta \ll O(1)$, we can write the solution of equation (2.15) as

$$
\psi=\psi^{(0)}+\beta \psi^{(1)}+\mathcal{O}\left(\beta^{2}\right)
$$

Substituting 2.16 into equation 2.15 and collecting terms with the same order of $\beta$ gives

$$
\begin{aligned}
& \left(\partial_{t}+\bar{v} \partial_{y}\right) \nabla^{2} \psi^{(0)}-\bar{v}_{x x} \psi_{y}^{(0)}=0 \\
& \left(\partial_{t}+\bar{v} \partial_{y}\right) \nabla^{2} \psi^{(1)}-\bar{v}_{x x} \psi_{y}^{(1)}=-\psi_{x}^{(0)}
\end{aligned}
$$

The zeroth order equation is the same equation for a jet on an $f$ plane, and 
has the unstable sinuous mode and varicose mode. The small correction $\psi^{(1)}$ slightly modifies the two basic modes to reflect the $\beta$ influence. The first order equation shows that the two basic zero-order modes have a forcing effect on the order one term $\psi^{(1)}$. The $x$-derivative leads to a 90 degree phase shift, so that the sinuous (varicose) mode imposes an antisymmetric (symmetric) forcing structure. As a result, the zeroth order modes lose their original symmetry. One example for the sinuous mode is shown in Figure 2-10.

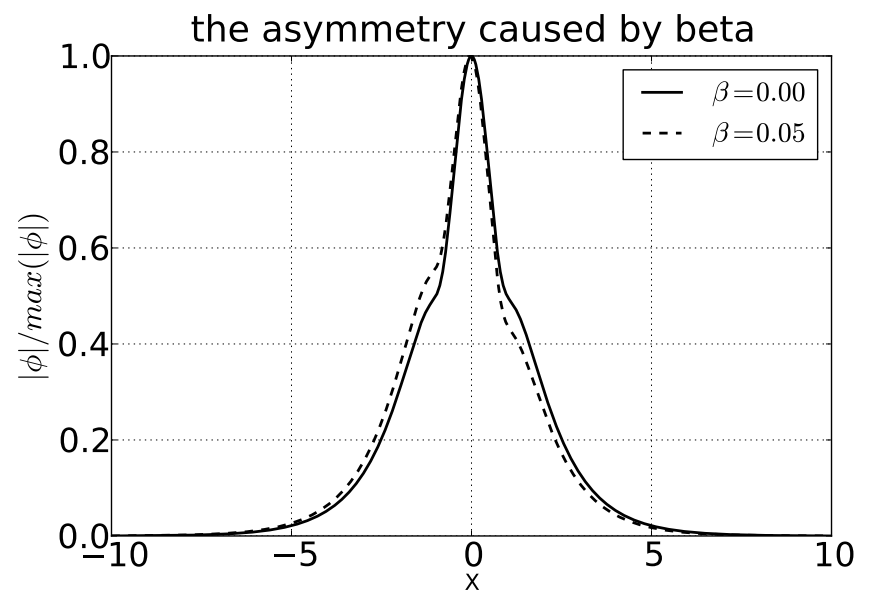

Figure 2-10: The cross-stream structure of the sinuous mode with $l=0.91$ for the f plane case (solid line) and the $\beta=0.05$ case (dashed line). The amplitude is normalized to have the maximum value equal one. An asymmetry in the cross-stream direction is introduced by the beta effect.

This asymmetry can be explained by diagnosing the energy budget for the perturbation. The energy budget for a uniform meridional jet is

$$
\frac{\partial}{\partial t} \int_{-L}^{L} d y \frac{1}{2}\left(u^{2}+v^{2}\right)=-\int_{-L}^{L} d y \overline{u v} \frac{\partial \bar{v}}{\partial x}
$$

The eigenfunctions in cases with $\beta \neq 0$ have asymmetric structures, and can be 
split into symmetric and antisymmetric components:

$$
\begin{aligned}
\psi^{(s)}(x) & =\frac{\psi(x)+\psi(-x)}{2} \\
\psi^{(a)}(x) & =\frac{\psi(x)-\psi(-x)}{2}
\end{aligned}
$$

Then the energy input can be decomposed into

$$
-\int_{-L}^{L} d y u v \bar{v}_{x}=-\int_{-L}^{L} d y\left(u^{s} v^{s}+u^{a} v^{a}+u^{s} v^{a}+u^{a} v^{s}\right) \bar{v}_{x}
$$

where the velocities are

$$
(u, v)^{(a \mid s)}=\widehat{k} \times \nabla \psi^{(a \mid s)} .
$$

The domain integration of the energy budget gives

$$
<-u v \bar{v}_{x}>=-<\left(u^{s} v^{s}+u^{a} v^{a}\right) \bar{v}_{x}>
$$

in which we omit the cross-correlation of the symmetric and antisymmetric terms as they have zero net-contribution to the total energy budget.

Figure 2-11 shows the decomposition of the energy input into the different Reynolds stresses. The black solid lines represent the calculation based on the $f$ plane case. They are symmetric about the origin. The red solid lines represent the total energy budget for the case with $\beta=0.05$. The interactions between symmetric and antisymmetric components $\left(u^{a} v^{s} V_{x}, u^{s} v^{a} V_{x}\right)$ introduce the asymmetry but do not contribute to the total integrated energy budget.

Let us first discuss the sinuous mode shown in the upper panel. There is an obvious asymmetry in the beta plane case shown by the red solid line. The left peak intensified but the right peak shrinks. The domain-integrated total energy input is calculated and shown by the values of $R_{\beta}$. The total energy input decreases from 0.093 to 0.088 when $\beta$ changes from 0 to 0.05 . By scrutiny, we can find that the decrease in the total energy input is mainly caused by the decrease in the sinuous 
mode itself (shown by the difference between the blue and the black lines). There is a non-zero energy contribution from the antisymmetric component (the green line) which is a projection of the varicose mode, but the compensation is not large enough to surpass the loss in the sinuous mode. The overall effect is that the energy input to the sinuous mode decreases, which leads to a smaller growth rate.

The effect on the varicose mode is shown in the lower panel. The same argument used for the sinuous mode can also be used for the varicose mode. The difference is that the total energy input for the varicose mode increases from 0.07 to 0.075 as $\beta$ increases from 0 to 0.05 . By comparing the green and the black lines, we notice that the varicose mode also loses energy. However, the energy input by the symmetric components (the blue dashed line) surpasses the energy loss. Then the overall effect for the varicose mode is that the growth rate is increased.

The asymmetric effect can be explained by the same mechanism used in explaining Rossby waves. Figure 2-12 sketches the two-dimensional structure of the streamfunction for the sinuous mode (left) and the varicose mode (right). If we include $\beta$ in the problem, a particle will obtain additional vorticity as it moves north and south. The sinuous mode has an antisymmetric structure in the meridional velocity, which induces a symmetric meridional velocity tendency (left panel, dashed arrows point to the same direction). For the varicose mode in the right panel, the symmetric meridional velocity structure induces an antisymmetric meridional velocity tendency (colored arrows point to opposite directions). Mathematically, this effect is represented by $v=\phi_{x}$, as the $x$-derivative interchanges the symmetry and the antisymmetry. This structure change leads to an overlap of the sinuous and varicose modes. The overlap of these two modes causes the increase of the growth rate for the varicose mode and the decrease for the sinuous mode.

We can also use the matrix perturbation method to calculate the asymptotic solution to illustrate this phenomenon. 

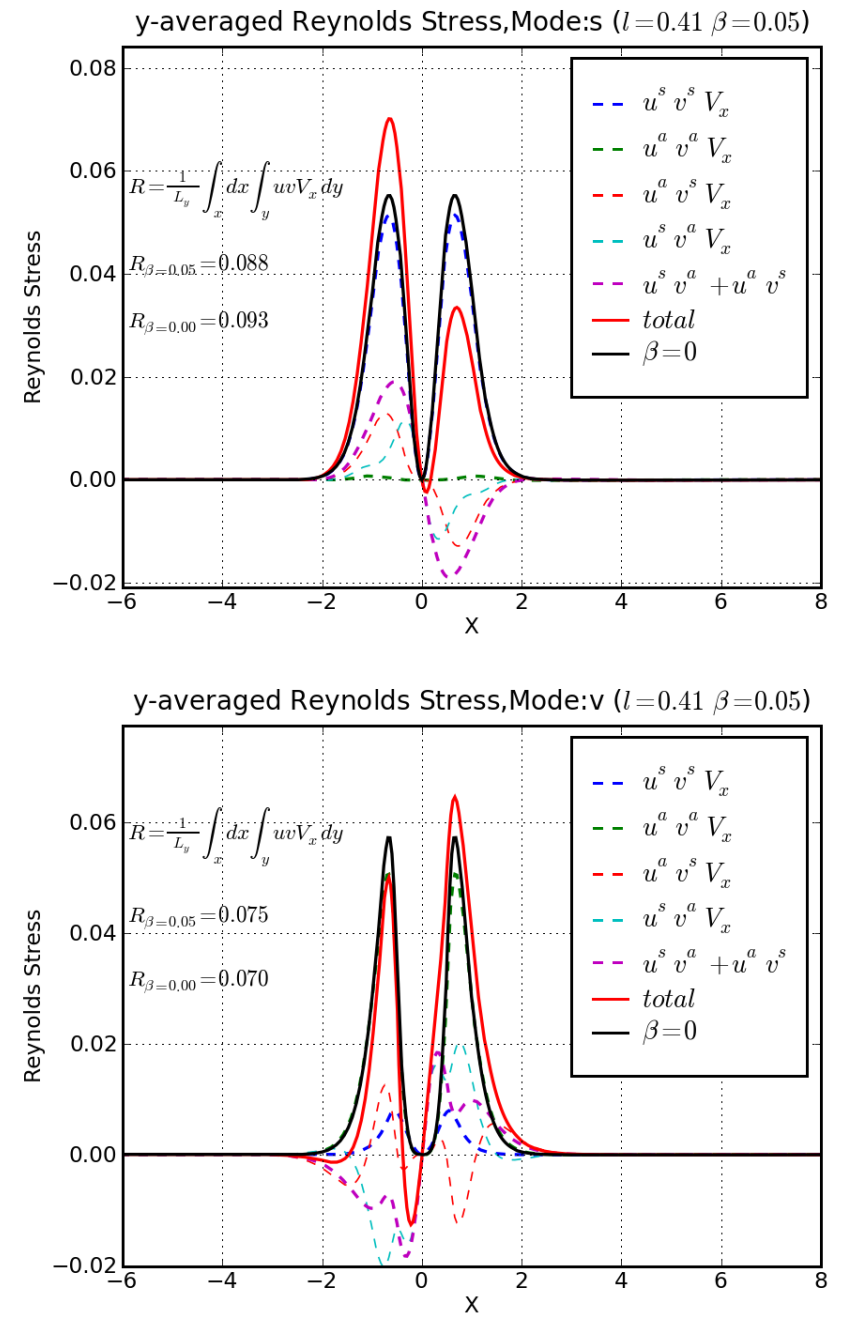

Figure 2-11: The energy input decomposition for the sinuous mode (the upper panel) and the varicose mode (the lower panel). The black lines show the $\beta=0$ case, with a symmetric structure about the jet center. $\beta$ introduces asymmetry into the Reynolds stress (solid red lines). The cross product of the symmetric and antisymmetric terms has no net energy contribution, but is the cause of the asymmetry. The beta effect reduces the energy input from the component that resembles the two mode's own structure (compare black and blue in the upper panel, and black and green in the lower panel). However, for the sinuous mode, the antisymmetric part has negligible energy contribution, while the contribution of the symmetric part to the antisymmetric varicose mode is much larger. The net effect is that the growth rate for sinuous mode decreases, but the growth rate for the varicose mode increases, which is shown by values of $R$ for $\beta=0$ and $\beta=0.05$. 

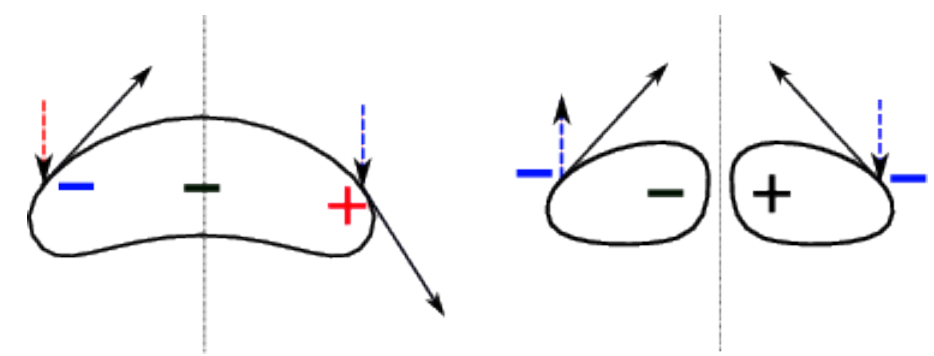

Figure 2-12: The beta induced asymmetry. The black contours represent the streamlines of a sinuous mode (left panel) and a varicose mode (the right panel). The black arrows represent the velocities which are tangential to the streamlines. The colored sign symbols represent the vorticity anomalies induced by the planetary beta and the particle movement in the direction shown by the black arrows. The colored arrows represent the velocity tendencies for particles at the location of the arrows caused by the anomaly on the other side of the jet center. The jet center is indicated by the solid vertical straight lines. Refer to the text for the detailed discussion.

The stability equation for the meridional jet is

$$
\left(\bar{v}\left(\partial_{x x}-l^{2}\right)+\frac{\beta}{i l} \partial_{x}-\bar{v}_{x x}\right) \phi=c\left(\partial_{x x}-l^{2}\right) \phi
$$

Rearranging it gives

$$
\left(\bar{v}\left(\partial_{x x}-l^{2}\right)-\bar{v}_{x x}\right) \phi=c\left(\partial_{x x}-l^{2}\right) \phi+i \beta l^{-1} \partial_{x} \phi
$$

The discretized form can be written as

$$
M_{1} \phi=c M_{2} \phi+\beta M_{3} \phi
$$

where $M_{(1,2,3)}$ are the finite difference matrices for the linear operators $\left[\bar{v}\left(\partial_{x x}-l^{2}\right)-\right.$ $\left.\bar{v}_{x x}\right],\left[\partial_{x x}-l^{2}\right]$, and $\left[i l^{-1} \partial_{x}\right]$. Assuming $\beta \ll O(1)$, we expand the eigenvalue and the eigenfunction using $\beta$

$$
\phi_{n}=\phi_{0 n}+\beta \phi_{1 n}+\beta^{2} \phi_{2 n}+O\left(\beta^{3}\right), c_{n}=c_{0 n}+\beta c_{1 n}+\beta^{2} c_{2 n}+O\left(\beta^{3}\right)
$$

where $n$ represents the $n^{\text {th }}$ mode, and $0,1,2$ represent the order of the solution in 
terms of $\beta$. The equations for the zeroth, the first and the second orders are

$$
\begin{aligned}
& \left(M_{1}-c_{0 n} M_{2}\right) \phi_{0 n}=0 \\
& \zeta_{0 n}^{T}\left(M_{1}-c_{0 n} M_{2}\right)=0 \\
& \left(M_{1}-c_{0 n} M_{2}\right) \phi_{1 n}=c_{1 n} M_{2} \phi_{0 n}+M_{3} \phi_{0 n} \\
& \left(M_{1}-c_{0 n} M_{2}\right) \phi_{2 n}=c_{1 n} M_{2} \phi_{1 n}+c_{2 n} M_{2} \phi_{0 n}+M_{3} \phi_{1 n}
\end{aligned}
$$

where $\zeta_{0 n}$ and $\phi_{0 n}$ are the $n^{\text {th }}$ left and right eigenvectors of the generalized eigenvalue problem $M_{1} x=c M_{2} x$, corresponding to eigenvalues $c_{0 n}$. The zeroth order equation is solved using a finite difference numerical method. After some algebra presented in the appendix, we get higher order solutions expressed by the zeroth order solution.

$$
\begin{aligned}
c_{1 n} & =0 \\
c_{2 n} & =-\sum_{k, k \neq n} \frac{1}{c_{0 k}-c_{0 n}} \frac{\zeta_{0 n} M_{3} M_{2}^{-1} M_{3} \phi_{0 n}}{\zeta_{0 n} \phi_{0 n}}
\end{aligned}
$$

If calculated based on a single $k, c_{2 n}$ represents the influence of the $k$-th mode on the $n$ th mode. The summation then represents the integration of all other modes' influences on the $n$-th mode. It is clear that once we have the zeroth order eigensolutions, which represent the solutions on an $f$ plane, we can use this formula to construct the solution for a problem with a small beta. As the first order correction $c_{1 n}=0$, the growth rate for the $n$-th mode then becomes

$$
c_{n}=c_{0 n}+\beta^{2} c_{2 n}
$$

which indicates that the sign of beta does not affect the growth rates of a parallel meridional jet located in the center of the domain.

Following the formula given in equation 2.34, we compute $c_{2 n}$ and plot the imaginary part of $c_{n}=c_{0 n}+\beta^{2} c_{2 n}$ which represents the growth rate in Figure 2-13.

The growth rate of the sinuous and the varicose mode are shown in the left and 

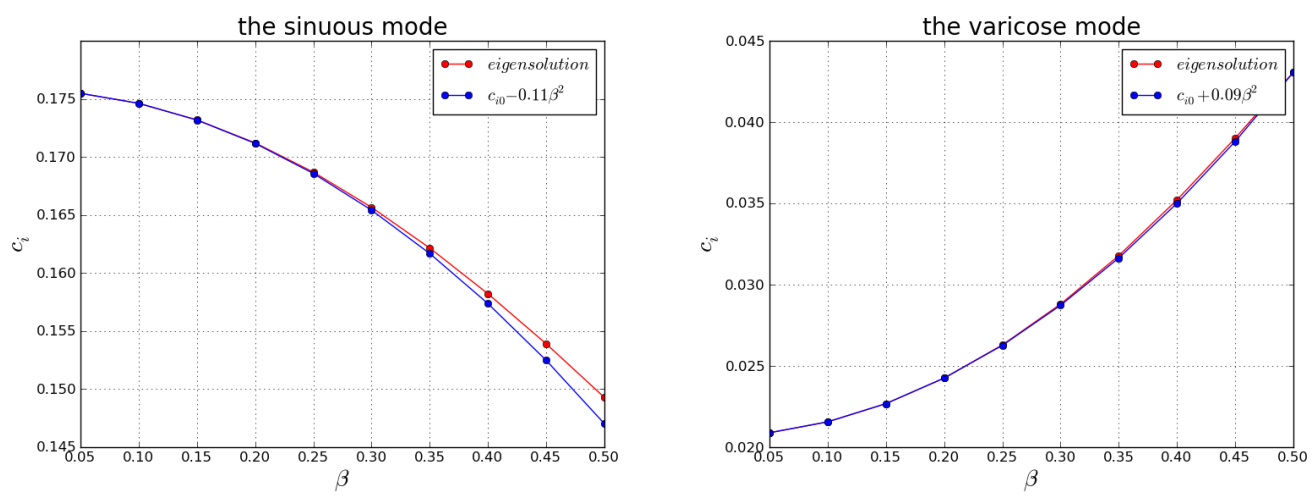

Figure 2-13: The relationship between the growth rate and $\beta$. The red lines represent the numerical solution of the eigenvalue directly calculated with $\beta$. The blue lines show the results calculated according to the perturbation theory (equation 2.35). The left panel shows that the growth rate of the sinuous mode decreases when $\beta$ increases. The right panel shows the opposite trend for the varicose mode. The good agreement between the red line and the blue line when $\beta$ is small validates of the perturbation theory, and confirms that the growth rate for the two growing modes has a quadratic relationship with the small $\beta$.

right panel respectively. The growth rate closely follows the parabolic line within the small $\beta$ range, and slightly drifts away when $\beta$ becomes large. The higher order correction of the growth rate is negative for the sinuous mode, but positive for the varicose mode. In this figure, the higher order correction is calculated as the sum of all other modes' influence. Next we test the idea of the sinuous-varicose interaction mechanism, which states that the projection of one mode on the other causes the growth rate to increase or decrease. We isolate the interaction between the two modes by choosing $n$ and $k$ to represent the sinuous and varicose mode. Figure 2-14 shows the interaction. We can see that the two-mode interaction is the main reason for the different behavior, i.e., the growth rate increases for the varicose mode but decreases for the sinuous mode. The two-mode interaction, however, can not explain all the changes of the growth rate for large betas, which means that other modes also have a projection on the two modes and contribute to the overall changes in the growth rates. 

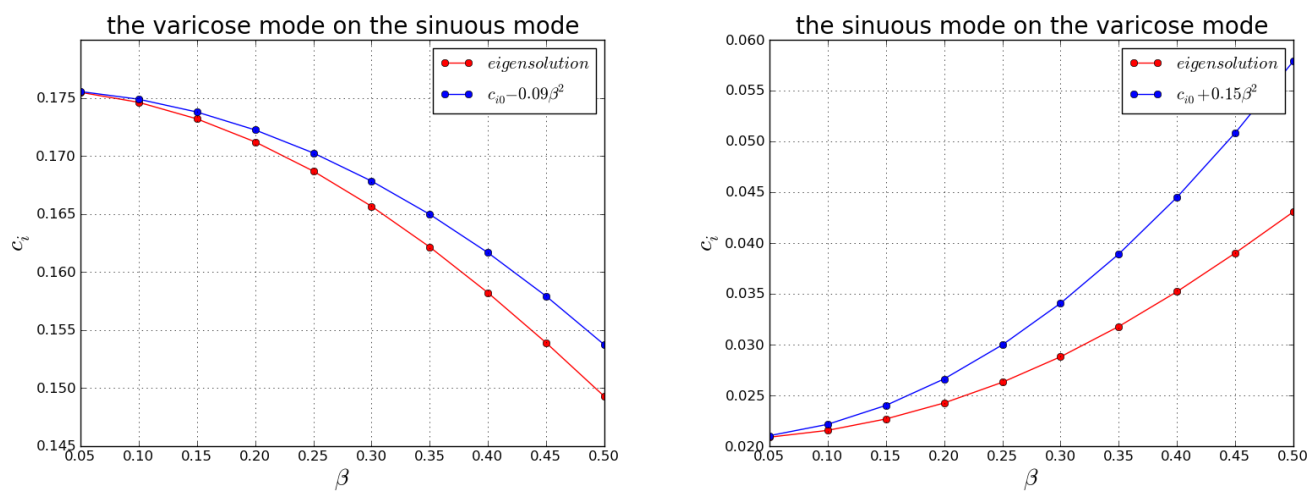

Figure 2-14: This figure shows the change in the growth rate caused by the two-mode interaction. Here the two modes we choose are the unstable varicose and sinuous mode. The skew-symmetric operator $M_{3}$ can modify two originally orthogonal eigenvector to invoke the mode interaction. The blue lines show the growth rates corrected by the two-mode interaction. The red lines are the solutions directly computed from the beta plane eigenvalue problem. The left panel shows the influence of the varicose mode on the sinuous mode. It shows the same trend as shown by the red line. Also we can observe the same phenomenon from the right panel. The blue and red lines are not in an excellent agreement especially for large betas, which means other modes in the zeroth order solution also contribute to the interaction.

\section{Unbounded meridional Bickley jet}

Whether waves can propagate through the domain boundaries distinguishes the bounded and the unbounded problem. It is rather complicated for the finite difference method to implement the radiating boundary conditions. In this section, we use a shooting method to implement the radiating boundary conditions and study the radiating instabilities of a meridional Bickley jet in an unbounded domain.

Previous studies show that a slight tilt of a zonal jet has a destabilizing effect (Walker and Pedlosky, 2002). The non-zonality incites the long wave radiation, which implies that the radiating instabilities of the swift boundary currents can transfer energy into the ocean interior. In Kamenkovich and Pedlosky (1996), the non-zonality is introduced by a meridional tilt of the zonal jet. After orienting the $x$ axis along the jet, the barotropic potential vorticity equation becomes

$$
\frac{\partial}{\partial t} \nabla^{2} \psi+J\left(\psi+\Psi(y), \nabla^{2} \psi+Q\right)=\mathcal{F}
$$


and the unperturbed state is given by

$$
Q=\beta_{1} x+\beta_{2} y-\bar{u}_{y} ; \Psi(y)=-\int \bar{u} d y
$$

where $\beta_{1}=\beta \sin (\alpha)$, and $\beta_{2}=\beta \cos (\alpha)$ with $\alpha$ being the tilt angle. The radiating instability comes from the new $\beta$ component normal to the jet, i.e. $\beta_{1}$.

The method was suitable for the investigation of the effect of the non-zonality on the jet instability and its associated wave radiation. We notice, however, that varying the tilt of the zonal jet $\alpha$ changes both $\beta_{1}$ and $\beta_{2}$. The destabilizing effect is a mixed result of increased $\beta_{1}$ and reduced $\beta_{2} . \beta_{2}$ is equivalent to the conventional $\beta$ in the pure zonal jet problem, and has a stabilizing effect on the jet. We are more interested in the influence of the meridional component, and try to isolate the effect of $\beta_{1}$. A straightforward way is to rotate the jet 90 degrees to set it meridional, for which $\beta_{2}=0$ and $\beta_{1}=\beta$.

One may argue that a pure meridional jet in a region without any adjacent lateral boundaries is simply unrealistic in either the atmospheric or oceanic system. What we want to show here is that the component of the background vorticity gradient perpendicular to the direction of the mean flow plays an important role in the instability of the current and its radiation. The results are expected to agree with those in Kamenkovich and Pedlosky (1996) by projecting $\beta$ for a meridional jet back to the $\beta_{1}$ for a tilted zonal jet.

On the subject of the instability of a meridional jet, previous studies focused on a boundary current, and used broken line profiles (Fantini and Tung, 1987; Hristova et.al, 2008). Fantini and Tung (1987) found that unstable waves generated by the meridional boundary jet are able to propagate energy away even in the presence of a realistic dissipation. They proposed that the shear energy of a western boundary current can be transported large distances into the ocean interior. Hristova et al. (2008) found that there is an asymmetry between a WBC and an EBC. An EBC supports a greater number of radiating modes over a wider range of meridional wavenumbers. 
Here we represent the jet using the Bickley profile, and study its instability radiation for unbounded and semi-bounded cases. The semi-bounded domain has one solid boundary and one radiating boundary in the zonal direction. The shooting method is described in the methodology section. The criteria used in Talley (1983) and Kamenkovich and Pedlosky (1996) to determine the radiating mode are also used in this study.

The first criterion is that a radiating mode will 'look' wavy in space (Talley 1983). It is easy to understand the meaning of the radiating mode by considering the structure of a neutral mode in the region with no mean flows. If the mode has the form of a plane wave, then it is a radiating one. If the mode decays in space away from the jet, we call it a trapped mode. In a region far away from the jet, the stability equation reduces to an ordinary differential equation with constant coefficients, and the solution is assumed to take the form

$$
\psi(x, y, t)=A e^{l c_{i} t-k_{i} x} e^{i l\left(x-c_{r} t\right)+i k_{r} x}
$$

where $c, k, l$ satisfy the dispersion relation of the ODE, the Rossby dispersion relation in this case. The wavy solution has the spatial decay scale $1 / k_{i}$ larger than the oscillation scale $1 / k_{r}$, so that $k_{i} / k_{r}<1$. However, this is not a sufficient condition. Spatial trapping does not necessarily rule out the possibility of radiation.

One thought experiment to demonstrate the uncertainty of this criteria is to consider an unstable radiating mode with a very large growth rate but a small radiation rate (i.e., the group velocity). The unstable mode extracts energy from the mean and grows exponentially to form a shape of trapped mode. If the radiation is fast enough to distribute the energy to the far field, we can easily recognize its radiating feature. However, if the radiation is a slow process comparing with the growth rate, the energy accumulates fast and the mode exhibits an exponential decay structure without showing wave structure. Under this situation, we might consider this mode as a trapped one. 
As a result, the second criterion to verify whether a solution is a radiating one is to study the behavior of the wavenumber in the limit $c_{i} \rightarrow 0$. If both $k_{r} \rightarrow 0$ and $k_{i} / k_{r} \rightarrow 0$, then we can claim that the spatial decay of the solution is due only to the nonzero values of the growth rates $l c_{i}$, and the solution is not trapped.

The third criterion is the so-called phase speed condition (McIntyre and Weissman, 1978; Talley, 1983; Malanotte-Rizzoli et al., 1987; Ierley and Young, 1991). The frequency and wavenumber of the solution of the linear eigenvalue problem must match those of the Rossby wave in the far field in order for the unstable mode to radiate.

The stability equation for a parallel meridional current is

$$
\left(\partial_{t}+\bar{v} \partial_{y}\right) \nabla^{2} \psi+\beta \psi_{x}-\bar{v}_{x x} \psi_{y}=0
$$

with $\bar{v}=-\operatorname{sech}^{2}(x)$. The perturbation is represented by normal modes

$$
\psi=\phi(x) e^{i l(y-c t)}+\text { c.c. }
$$

where $l$ is the meridional wavenumber, $c$ the meridional phase speed, and c.c. the complex conjugate. Substituting the normal mode solutions into the stability equation, we get the following eigenvalue problem

$$
\partial_{x x} \phi+\frac{\beta}{i l(\bar{v}-c)} \partial_{x} \phi-\left(l^{2}+\frac{\bar{v}_{x x}}{\bar{v}-c}\right) \phi=0
$$

which is solved using the shooting method.

Given a real meridional wavenumber $l$, we make an initial guess of the possible eigenvalue $c$. The eigenvalue equation is integrated zonally from the western far field to the eastern far field. The boundary conditions are important in the calculation.

The boundary condition in the far field is the radiation condition, represented by $\phi_{x}+i k \phi=0$, in which $k$ is the zonal wavenumber. The zonal wavenumber, together with the given meridional wavenumber $l$ and the guessed phase speed $c$, satisfy the 
free Rossby wave dispersion relationship

$$
l c=-\frac{\beta k}{k^{2}+l^{2}}
$$

in the far field where the basic state $\bar{v}=0$. As a result, the zonal wavenumbers are calculated by solving this second-degree equation. The solutions for the zonal wavenumber are pair of complex conjugates with opposite-signed imaginary parts for each set of meridional wavenumber $l$ and eigenvalue $c$. We should pick such a zonal wavenumber that the energy is radiated away from the source region. It can be shown that in the weakly unstable limit, the sign of the imaginary part of the zonal wavenumber, $k_{i}$, relates to the direction of the Rossby wave group velocity (Hristova et al., 2008; Fantini and Tung, 1987). The zonal wavenumber with a positive/negative imaginary part is associated with a boundary condition of eastward/westward energy radiation, so that $k_{i}>0$ is applied to the eastern boundary and $k_{i}<0$ to the western boundary.

After the zonal wavenumber is chosen for each boundary condition, the eigenvalue equation is then integrated from the western boundary to the eastern boundary using a 4th-order Runge-Kutta method. The direction of the integration does not change the eigensolutions. The difference between the value of $\phi$ and $i k \phi$ at the end of the integration forms a discrepancy function, by minimizing which the eigenvalue problem is solved.

We use $L_{x}$ to represent the eastern side of the calculation domain and $-L_{x}$ the western side hereafter. $x=0$ is set as the domain center where the jet is located. The zonal wave number is $k_{1}$ at $x=-L_{x}$ and $k_{2}$ at $L_{x}$. In the following, we discuss the growth rate, phase speed, zonal wavenumber, and radiating features separately.

For an unbounded meridional jet, the instabilities are able to propagate in both zonal directions. As we can speculate, the planetary $\beta$ creates an asymmetry in the zonal instability radiation. 
Growth rate: Figure 2-15 shows the growth rate of the unbounded centered meridional jet with $\beta=0.1,0.2$. Several features are similar to the eigensolution for a bounded meridional jet. Most of the beta effect appears over the longwave end. The wavenumber for the neutral varicose mode is less than one, and is less than two for the sinuous mode. The beta effect reduces the growth rate of the sinuous mode, but increases the growth rate of the varicose mode. The longwave cutoff appears for both modes. Beta has little influence on the short sinuous mode. In this unbounded case, however, there are no other modes except for the sinuous and varicose modes, which is opposite to the bounded case. It demonstrates that the reflected waves are responsible for other slowly growing modes in the bounded case.

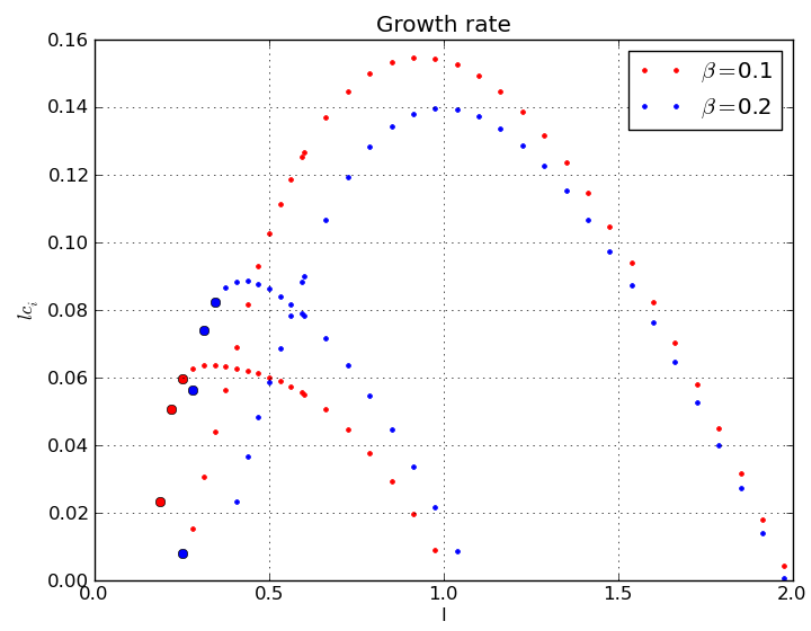

Figure 2-15: The growth rates for $\beta=0.1$ (red lines) and $\beta=0.2$ (blue lines). The dotted curves spanning between wavenumber 0 and 2 represents the sinuous mode, and the curves spanning between wavenumber 0 and 1 represent the varicose mode. The beta effect decreases the growth rate of the sinuous mode, but increases the growth rate of the varicose mode.

Phase speed: Figure 2-16 shows the phase speed of the varicose mode (the lower two curves) and the sinuous modes (the upper two curves). Both modes have negative phase speeds, meaning they travel in the same direction with the jet $\left(\bar{v}=-\operatorname{sech}^{2}(x)\right)$. The varicose mode propagates faster than the sinuous mode. The change of the phase 
speed for the varicose mode as $\beta$ increases is different from the change for the sinuous mode. The beta effect accelerates the varicose mode but decelerates the sinuous mode. It is also true in terms of the phase speed that the beta influence is large for longwaves but small for short waves.

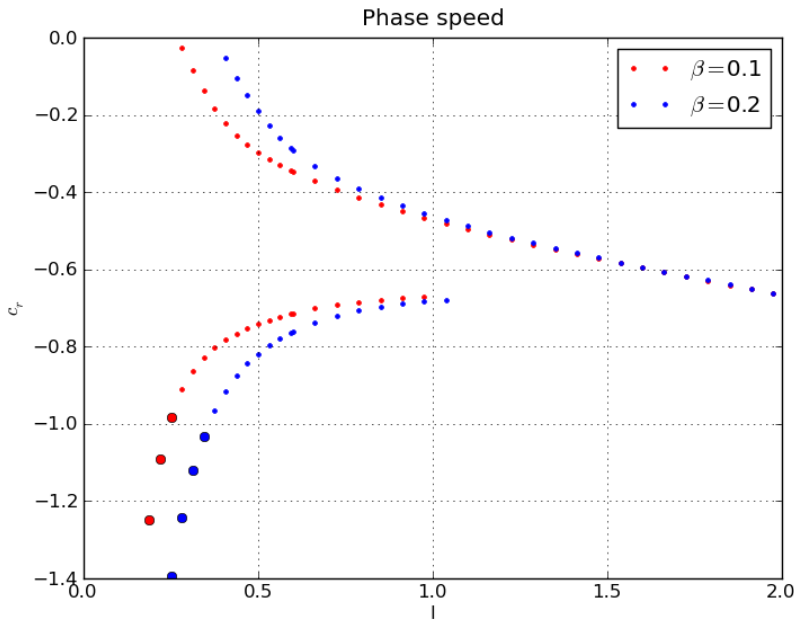

Figure 2-16: $c_{r}$ for $\beta=0.1$ (red lines) and $\beta=0.2$ (blue lines). The varicose modes are the two lines in the lower half of the figure. The upper two curves represent the sinuous mode.

Radiating modes: The varicose mode is more able to radiate. Figure 2-17 shows the value $k_{1 i} / k_{1 r}$ for different modes. The negative values indicate those radiating modes with wavy structures. When $\beta$ is small $(<0.1)$, only the varicose mode is able to radiate. When $\beta$ becomes large, however, the radiating modes start to emerge at the longwave end for the sinuous mode. This radiating sinuous mode only radiates westward. The right panel of figure 2-17 shows one example of the structure of the radiating sinuous mode. 

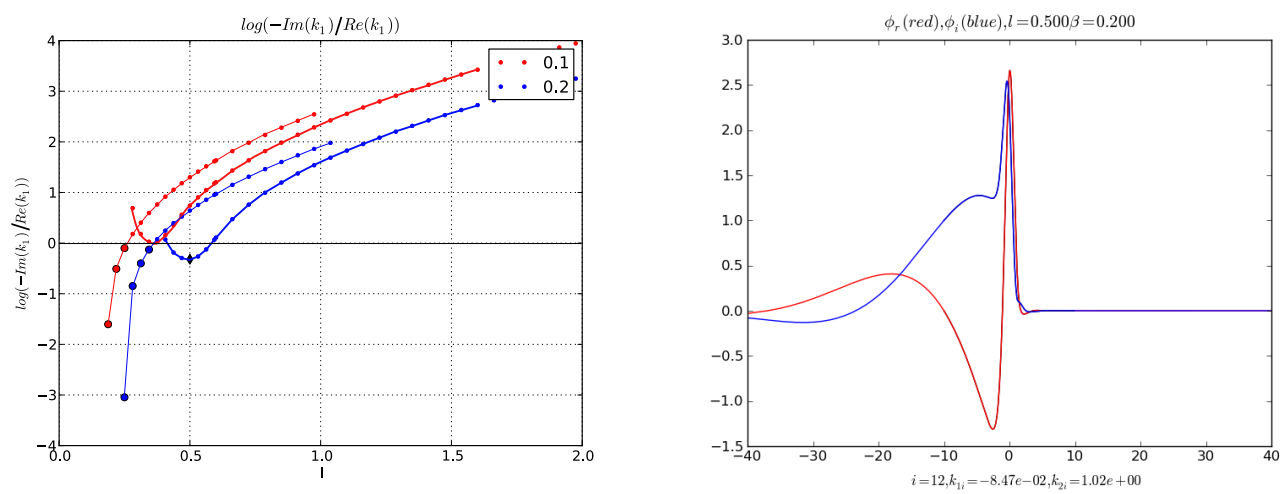

Figure 2-17: The left panel shows $\log \left(k_{1 i} / k_{1 r}\right)$ for $\beta=0.1$ (red) and $\beta=0.2$ (blue). The right panel shows the structure of the radiating sinuous mode indicated by the black dot in the left panel, red line is the real part and the blue line is the imaginary part.

In summary, we find that the unbounded meridional Bickley jet shares some features with the bounded one, and also exhibits some differences. The growth rate of the varicose mode increases but the growth rate of the sinuous mode decreases when $\beta$ increases, which is similar to what we found in the bounded case. The differences are that the bounded jet has more than two unstable modes among which the modified sinuous and varicose modes have the larger growth rates, while the unbounded jet has only two unstable modes. The existence of the slowly growing modes are generated by the boundary wave-reflection which is absent in the unbounded domain. When $\beta$ increases, the longwave cutoff appears, and the cutoff moves towards the shortwave end. Those short varicose modes with wavenumber slightly larger than one, which are stable for the zonal jet problem, start to grow when $\beta$ increases.

The $\beta$ effect increases the phase speed of the varicose mode, but retards the sinuous mode. The phase speed is larger than one for certain long waves (in terms of the meridional wavelength), meaning that the perturbation travels faster than the mean current. The increase of the phase speed for a WBC is also observed in previous studies (Fantini and Tung, 1987; Hristova et al., 2008). The response of the phase speed to the beta effect highlights the difference between the meridional jet and the zonal jet. 
The varicose mode is more able to radiate than the sinuous mode. The long waves for the varicose mode start to radiate when $\beta$ increases. When $\beta$ is relatively small, the sinuous mode does not radiate, but when $\beta$ becomes large, it exhibits a radiating property shown by the $\log \left(k_{1 i} / k_{1 r}\right)$ figure, but only in the westward direction. However, one caveat is that these results are for the Bickley jet, and may not be applied to other velocity profiles.

\subsubsection{The $\beta$ effect on meridional boundary currents}

The study by Hristova et al. (2008) shows that the stability of an EBC is different from that of a WBC. It is mainly caused by the different directions of the energy propagation for the long and short Rossby waves. In their study, a broken line velocity profile is used to represent the boundary jet. The broken line (piecewise) profile has the advantage that the stability equation is reduced to an ordinary differential equation with constant coefficients. The stability problem is then to convert the boundary and matching conditions into a homogeneous linear algebraic system, from which the eigenvalues are calculated. The disadvantage of a broken line profile is that shortwave behaviors can not be physically resolved. We extend their study by comparing the stability of an eastern and western boundary currents with a continuous velocity profile and exploring a wider $\beta$ space.

To simplify the problem, we use a half Bickley jet profile to represent the boundary jet. It is known from the results of previous sections that the varicose mode is the only unstable mode for a half Bickley jet. The unstable varicose mode for an $f$ plane problem has wavenumber between 0 and 1 . For a centered meridional jet, the beta effect leads to an interference between the sinuous and the varicose modes. As a result, the growth rate decreases for the sinuous mode but increases for the varicose one. However, the sinuous mode does not exist for a half Bickley jet profile, so that we expect different characteristics of the varicose mode under the $\beta$ influence.

We use the same shooting method to solve the eigenvalue problem in a semi-infinite domain. The solving procedure is the same as described in the previous section. The 
only difference is that the eigenvalue equation is integrated from the solid boundary where the no-normal-flow condition is applied. The boundary condition in the far field is still the radiation condition.

In the following, we first discuss the asymmetrical stability characteristics between the eastern and western boundary jet in terms of the growth rate, phase speed, and zonal structure, then discuss the instability radiation.

\section{Growth rates}

Figure 2-18 shows growth rates of the EBC and WBC as functions of $l$ and $\beta$. There is only one type of mode, which is the varicose mode as discussed above even for cases with nonzero $\beta$ s. This is different from the stability of a centered jet where sinuous and varicose modes interact.

There is an obvious asymmetry. When $\beta=0$, the EBC is identical to the WBC, so that they share the same curve of growth rates. When $\beta$ increases, the EBC supports fewer unstable modes with smaller growth rates, while the WBC supports about the same amount of unstable modes but with shorter wavelengths and larger growth rates. An increase of the nondimensional parameter $\beta$ is equivalent to a decrease of the jet strength as $\beta^{*}=\beta L^{2} / V$. We can infer from the growth rate asymmetry that equally weak currents are more stable along the eastern boundary than along the western boundary. The eigensolution in Figure 2-18 is confirmed by time-dependent numerical simulations shown by the white stars.

The WBC becomes more unstable following the increase of beta. The growth rate continuously increases. We extend the calculation to $\beta=0.9$, and the maximum growth rate still increases. We infer from this result that WBC is unstable for all values of $\beta$. However, further testing are needed to confirm the hypothesis.

In order to explain the asymmetry, we examine the structure of the streamfunction and the energy budget. Figure 2-19 shows the streamfunctions for the EBC and WBC in cases with $\beta=0.1$ and $\beta=0.15$. Recall that the barotropic instability is triggered 

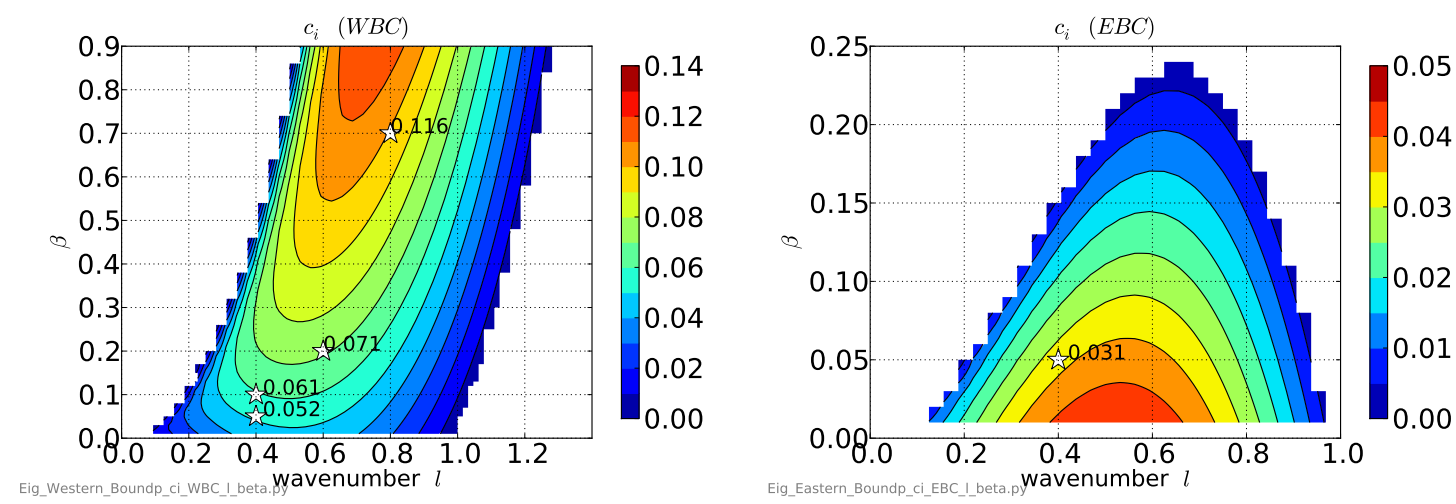

Figure 2-18: Growth rates for the EBC (right) and the WBC (left) as functions of the wavenumber and beta. When $\beta$ increases, the EBC supports fewer unstable modes with smaller growth rates, while the WBC supports about the same amount of unstable modes but with shorter wavelengths and larger growth rates. The white stars show the growth rates calculated from time-dependent numerical simulations for some randomly picked $(l, \beta)$ pairs. They are used to verify the eigenvalue solutions. More points are considered in the WBC case as it has a wider $\beta$ range.

by the phase tilt in the perturbation field. The perturbation has such a phase tilt that it extracts energy from the shear of the jet. The correlation between the phase tilt and the shear determines how efficiently the perturbation field extracts energy from the mean.

Figure 2-19 shows that the phase tilt changes for the perturbation field when $\beta$ changes. For the EBC (right panel), the phase tilt is more aligned with the mean shear. The growth rate is expected to be diminished. The situation is different for WBC, where the streamlines are tilted to become more against the shear, so that the perturbation grows faster.

A qualitative beta effect brought by the phase tilt can be shown by the energy budget. The domain integrated energy equation for a disturbance in a meridional jet is

$$
\frac{d<E>}{d t}=-<u v \bar{v}_{x}>
$$

where $E=\left(u^{2}+v^{2}\right) / 2$, and $<\cdot>$ represents the domain integration. The meridional mean of the Reynolds stress is shown in Figure 2-20. It confirms the above descriptive 

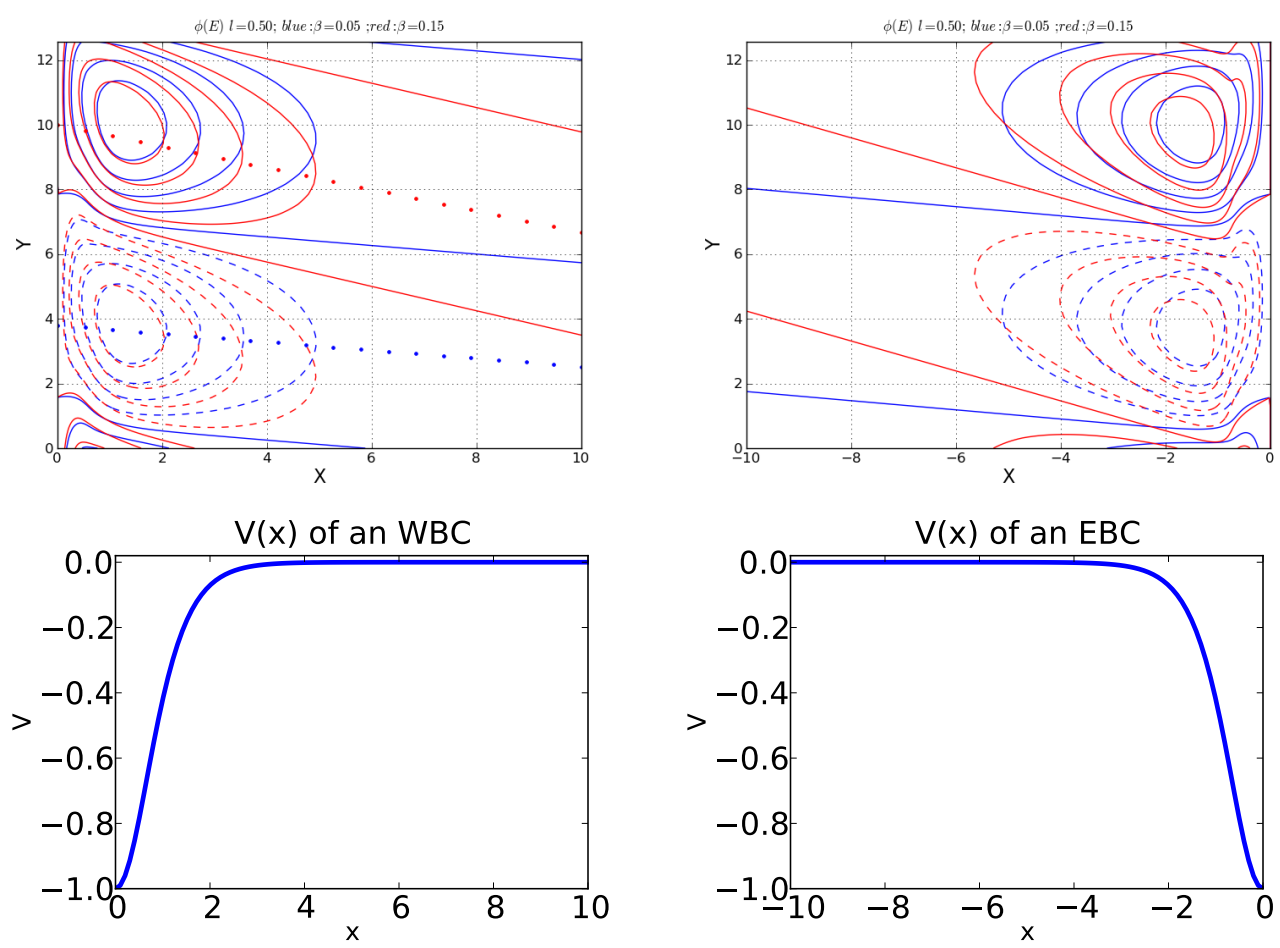

Figure 2-19: The streamfunctions of the perturbation at wavenumber $l=0.5$ for the $\mathrm{EBC}$ (right) and the WBC (left). Red (blue) lines represent the $\beta=0.15(\beta=0.05)$ cases. The black lines in the lower panels shows the jet profile (the half Bickley jet) for the eastern (right) and western (left) boundaries. The dotted lines indicate the slopes calculated by $-k / l$. By comparing two different pattern of the streamfunction, we can infer the mechanism responsible for the asymmetry shown in the growth rates, which is related to the phase tilt.

argument that the perturbations extract energy from the mean easier in the WBC than in the EBC. Although the energy transfer increases at some places in the EBC when beta increases, the energy loss is bigger than the gain, and the overall effect is that less energy is transferred from the mean. We should keep in mind that the growth rate represents the growth of the whole pattern of one mode. The horizontal advection can rearrange energy, and make the argument about the local energy balance less meaningful. The phase tilt, or the slope exhibited by the streamlines, is a key element in the energy transfer process.

This mechanism does not depend on one specific velocity profile. Another velocity profile, $\bar{v}=1 \mp \tanh (x), x=0 \cdots \pm L(-L$ for an EBC and $+L$ for a WBC), is tested. 

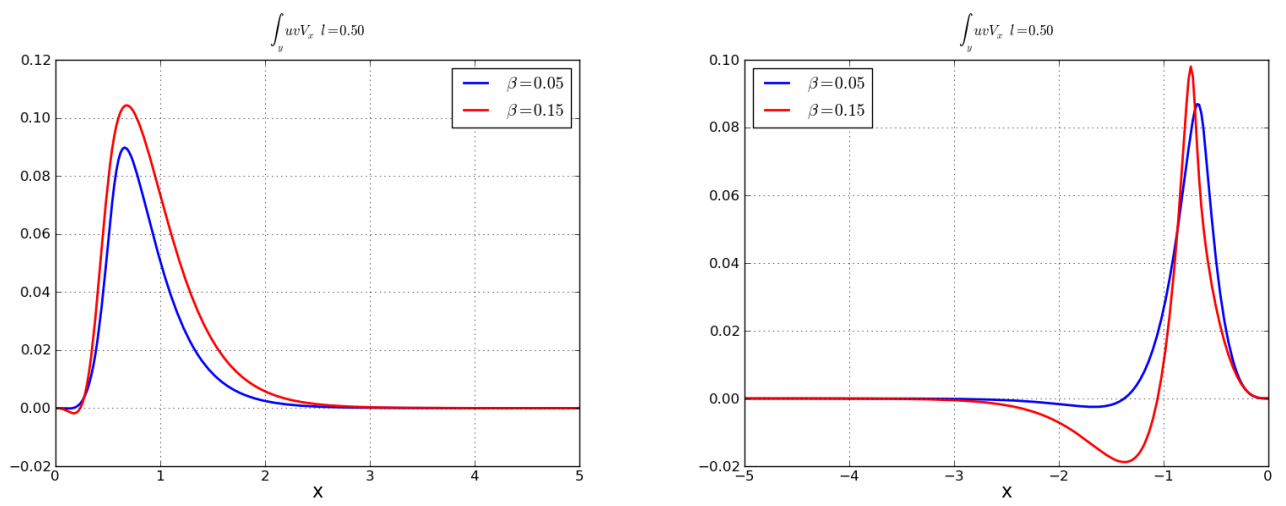

Figure 2-20: The energy transport from the mean to the perturbation, $-\int_{y} u v \bar{v}_{x} d y$. The $\beta$-modified stream-function in the WBC (left) has such a structure that more energy can be transferred from the mean to the perturbation. The opposite is true for the perturbation in the EBC (right).

It remains stable along the eastern boundary, but becomes unstable along the western boundary when $\beta$ increases. Notice that this profile is a half hyperbolic tangent with no inflection point, it is stable on an $f$ plane and also on a beta plane if the flow is zonal. The conventional stability criteria that is valid for the zonal jet can not be applied directly to the meridional jet.

\section{Meridional phase speed}

An increased phase speed of the perturbation along the WBC is reported in previous studies (Berloff and McWilliams, 1999; Fantini and Tung, 1987; Hristova et al., 2008). Berloff and McWilliams (1999) used a multi-layer QG model. Fantini and Tung (1987) and Hristova et al. (2008) both used the broken line velocity profile. In this study, we observe the same phenomenon with a meridional Bickley jet. Additionally, the phase speed of the perturbation along the EBC decreases following a $\beta$ increase. This is another asymmetry between the WBC and the EBC.

Figure 2-21 shows the phase speed of the perturbation in the EBC (right) and WBC (left). For a fixed beta, the phase speed is larger for long waves and smaller for short waves, which can be applied to both the WBC and EBC. For a fixed wavenumber, the phase speed becomes larger (smaller) when $\beta$ increases in the WBC (EBC) 
case. In the WBC case, the phase speed can become larger than one.
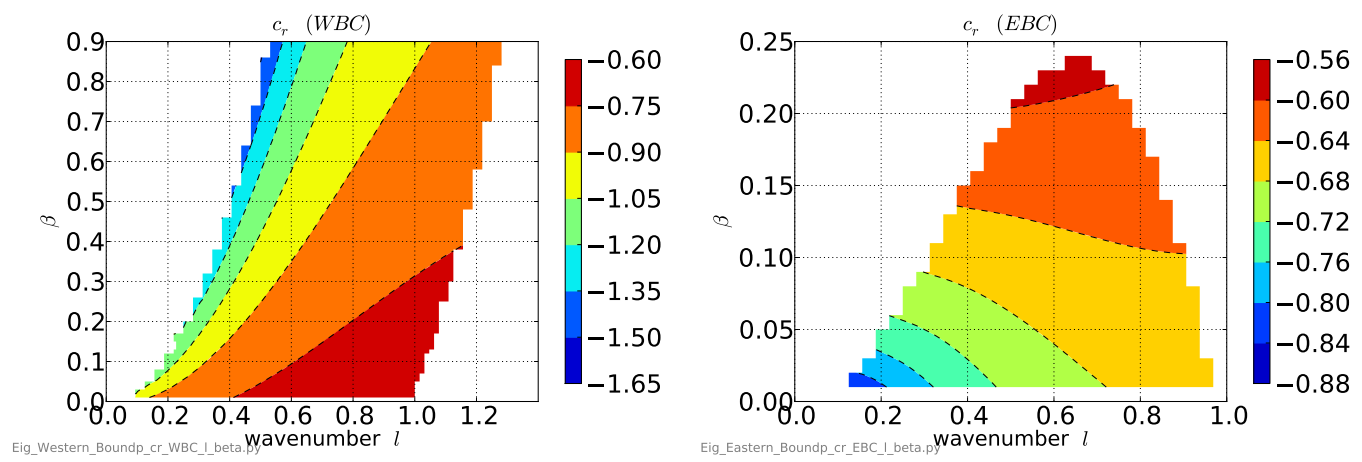

Figure 2-21: The meridional phase speed $c_{r}$ in the $(l, \beta)$ space for the EBC (right) and the WBC (left), in which $l$ is the meridional wavenumber. As $\beta$ increases, the phase speeds of the unstable modes increase in the WBC but decrease in the EBC.

\section{Zonal wavenumber}

Figure 2-22 shows the real part of the zonal wave number $k_{r}$ in the $(l, \beta)$ space for the $\mathrm{WBC}$ and $\mathrm{EBC}$. Both the $\mathrm{EBC}$ and $\mathrm{WBC}$ show the same zonal wavenumber characteristics. For a fixed meridional wavenumber, the zonal wavenumber for both the $\mathrm{EBC}$ and $\mathrm{WBC}$ increases following the increase of beta. Additionally, both the $\mathrm{EBC}$ and WBC show that larger meridional wavenumbers correspond to smaller zonal wavenumbers, and vice versa.

Fig $2-23$ shows $k_{r}$ as a function of $l$ with a fixed $\beta=0.1$. They show that $k_{r}$ of the unstable modes are smaller for the WBC than for the EBC given the same pair of $l$ and $\beta$. It means that given unstable modes with the same meridional wavelength, the one in the EBC has a smaller zonal wavelength than the one in the WBC. It seems to be contrary to our intuition and the conclusions in Hristova et al. (2008) that EBC radiates long Rossby waves with smaller decay rates, but the WBC radiates short Rossby waves with larger decay rates. They compared a pair of unstable modes, one for the $\mathrm{EBC}$ and another for the $\mathrm{WBC}$, with the same frequency. The comparison based on the fixed wavenumber may be misleading, but it raises an important issue in analyzing results. We consider both the meridional wavenumber and frequency to 
analyze the east-west asymmetry in the zonal wavelength.
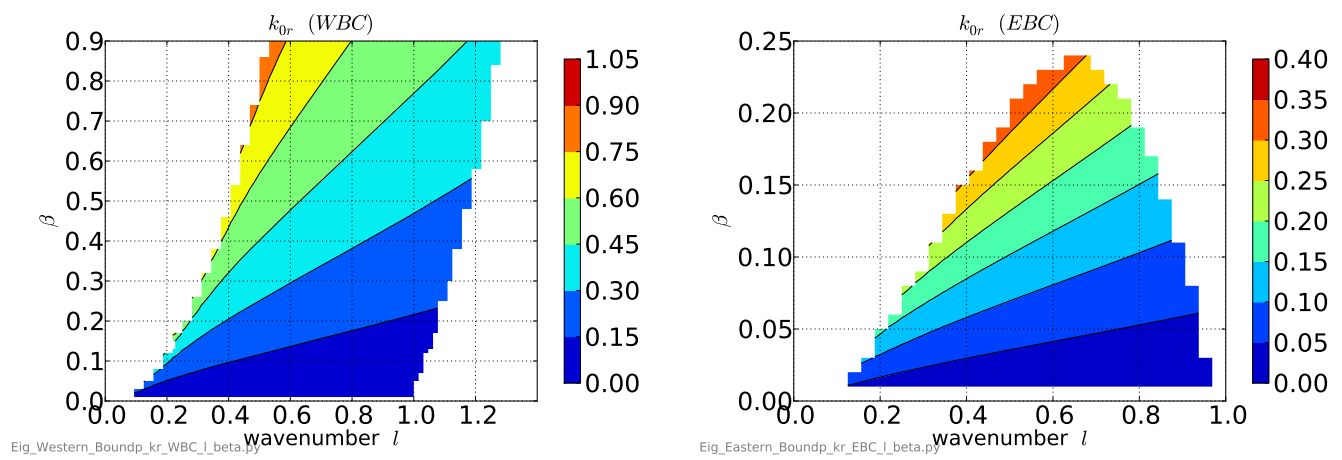

Figure 2-22: The zonal wavenumbers $k_{r}$ in $(l, \beta)$ space for the EBC (right) and the WBC (left).

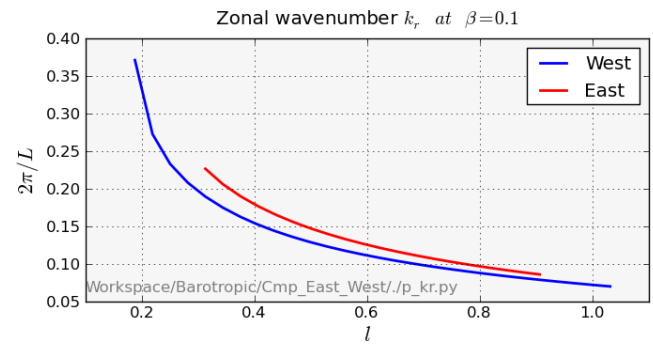

Figure 2-23: The zonal wavenumber $k_{r}$ at $\beta=0.1$. The red line is for the EBC and the blue line is for the WBC.

The phase speed increases in the $\mathrm{WBC}$ but decreases in the $\mathrm{EBC}$ when beta increases. This divergence of the phase speeds for a fixed wavenumber between the $\mathrm{EBC}$ and the WBC leads to the divergence of frequencies. Figure 2-24 shows the frequencies of the unstable modes in the $(l, \beta)$ domain for the EBC (right) and the WBC (left). For a fixed wavenumber, say $l=0.4$, when beta increases, the frequencies in the $\mathrm{EBC}$ decrease while those in the WBC increase. Let us now follow a fixed frequency, say $\omega=-0.3$ shown by the thick black dotted lines. For the $\beta=0$ case, the $\mathrm{EBC}$ is identical to the $\mathrm{WBC}$, and the unstable mode correspond to the wavenumber 0.4. When beta increases, the unstable mode with frequency -0.3 shifts to shorter wavelength in the $\mathrm{EBC}$ (right) but to the longer wavelength in the WBC (left), which 
is clearly shown by the slopes of the thick dotted lines. As the meridional wavenumber of the unstable modes has a monotonic relationship with the zonal wavenumber for both the WBC and EBC (Figure 2-23), the zonal wave becomes longer in the EBC but shorter in the WBC.
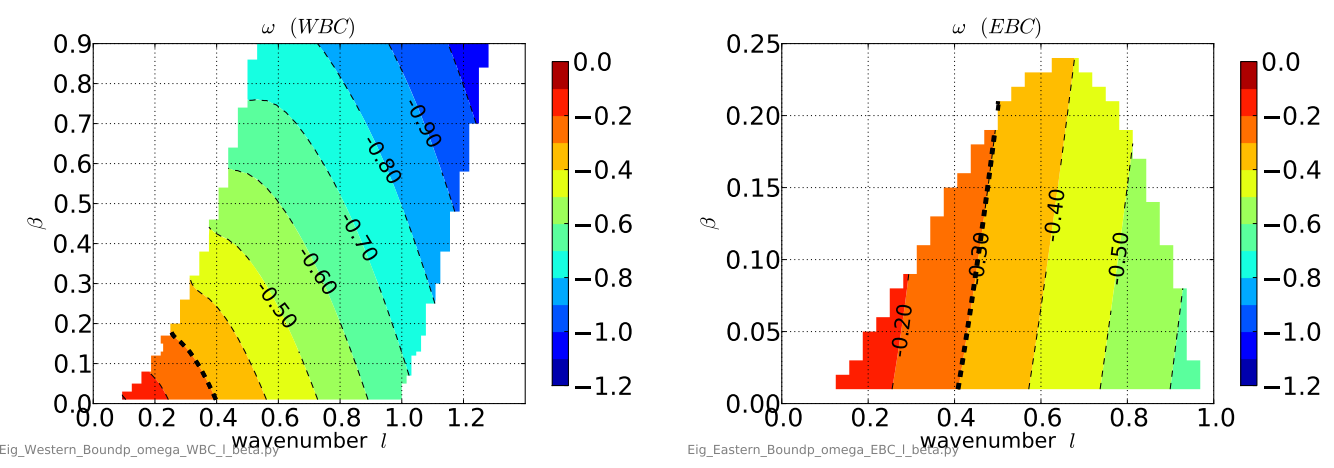

Figure 2-24: The frequency of the unstable modes for the EBC (right) and WBC (left). The thick dotted lines highlight the contours of the constant frequency -0.3 . As beta increases, it shifts to the right for the EBC but to the left for the WBC. In other words, it shifts to shortwaves for the EBC but to the longwaves for the WBC.

The process can be illustrated using the diagram of the Rossby wave dispersion relation. Taking into account that $k$ and $c$ are complex numbers, the real part of the Rossby wave dispersion relation can be written as

$$
\left(k_{r}+\frac{\beta c_{r}}{2 l|c|^{2}}\right)^{2}+l^{2}=\frac{\beta^{2} c_{r}^{2}}{2 l^{2}|c|^{4}}+k_{i}^{2}-\frac{\beta k_{i} c_{i}}{l|c|^{2}}
$$

For a given $c$, the wavevector lies on a circle whose center is at $\left(k_{r}=-\frac{\beta c_{r}}{2 l \mid c c^{2}}, l=0\right)$, and the radius is $\left(\frac{\beta^{2} c_{r}^{2}}{2 l^{2}|c|^{4}}+k_{i}^{2}-\frac{\beta k_{i} c_{i}}{l|c|^{2}}\right)^{1 / 2}$. If $c_{i}, k_{i}=0$, the relation returns to the classical energy diagram of Rossby waves. The meaning of the diagram becomes ambiguous with large $c_{i}$ and $k_{i}$. Nevertheless, it can illustrate that the zonal wavenumber is larger in the EBC than in the WBC given the same meridional wavenumber. The imaginary part of the equation is not discussed here.

Figure 2-25 shows the diagram for the WBC (blue) and the EBC (red) with $\beta=0.05$. The dots show the position of the unstable modes in the diagram for the $\mathrm{WBC}$ and $\mathrm{EBC}$. The zonal wavenumber is larger for the $\mathrm{EBC}$ than for the WBC given 


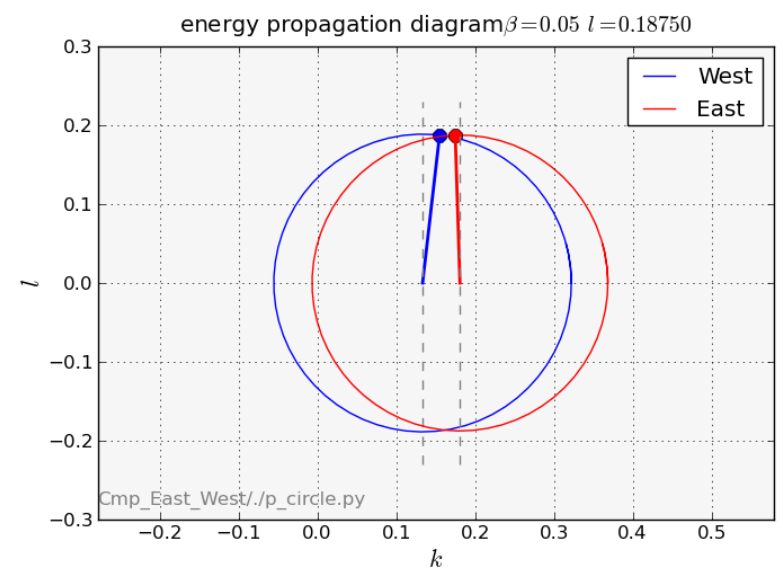

Figure 2-25: The diagram shows the Rossby wave dispersion relation with $\beta=0.05$. The circle center is at $\left(k_{r}=-\frac{\beta c_{r}}{2 l \mid c c^{2}}, l=0\right)$, and the radius is $\left(\frac{\beta^{2} c_{r}^{2}}{2 l^{2}|c|^{4}}+k_{i}^{2}-\frac{\beta k_{i} c_{i}}{l|c|^{2}}\right)^{1 / 2}$. The blue dot represents the $\mathrm{WBC}$, and the red dot represents the EBC. Both cases have $\beta=0.05$ and $l=0.1875$. The zonal wavenumber for the EBC is larger than that for the WBC even though the group velocity is negative for the former and positive for the latter. This is caused by shifts of the circles centers between the EBC and WBC.

the same $l$. This is caused by the shift of the circle centers, which are determined by both $\beta$ and $c$ which correlate to each other but with different signs for the WBC and EBC.

The boundary condition in the far field requires a structure that exponentially decays from the unstable region to the far field. Equivalently, $k_{i}$ is chosen to be negative for the EBC and positive for the WBC. Figure (2-26 and 2-27) show the property of $k_{i}$ as a function of $l$ and $\beta$. the EBC and WBC share similar features. The zonal decay rates $k_{i}$ are smaller for longer meridional waves and larger for shorter meridional waves. $k_{i}$ becomes smaller when $\beta$ increases. Given the same $\beta$ and $l$, the decay rate $k_{i}$ is larger for the WBC than for the EBC. 

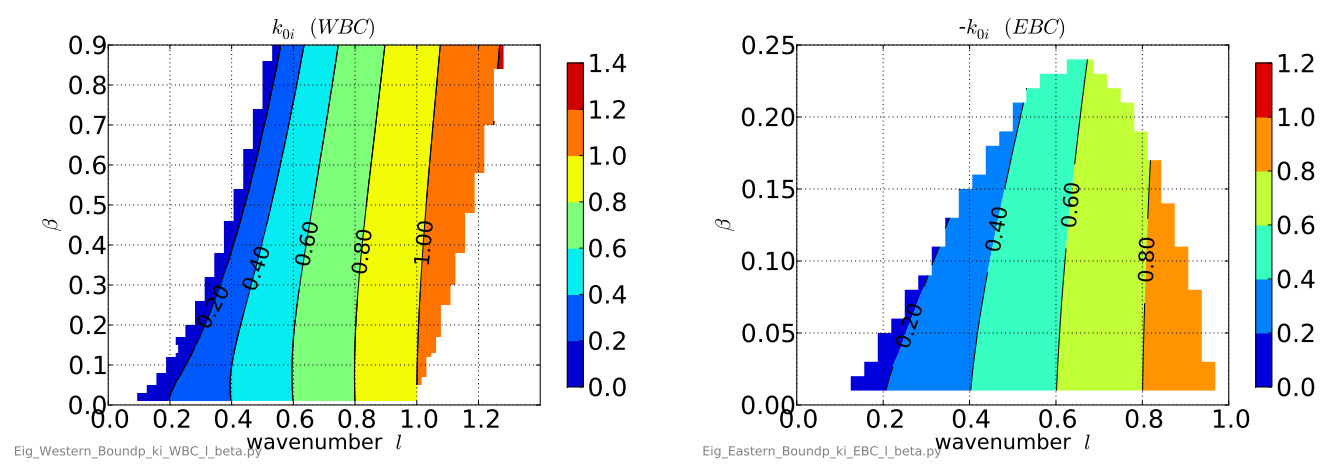

Figure 2-26: The zonal decay rates $k_{i}$ in $(l, \beta)$ space for the EBC (right) and WBC (left). $k_{i}$ is negative for the EBC, but $\left|k_{i}\right|$ is shown for the sake of easier comparison with those for the WBC.

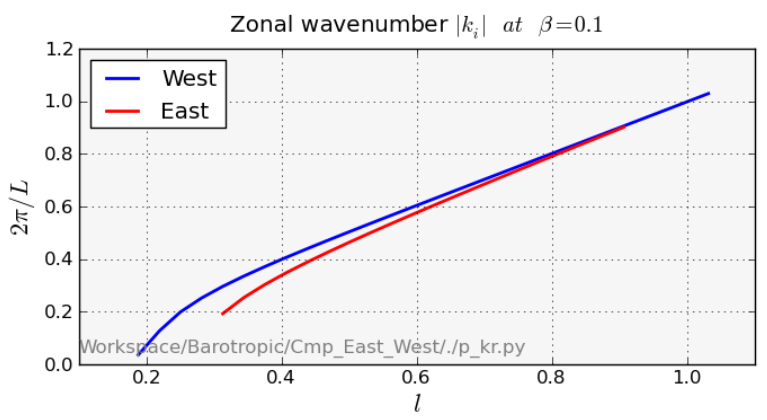

Figure 2-27: The zonal wavenumber $k_{i}$ at $\beta=0.1$. The red line is for the EBC and the blue line is for the WBC. The zonal decay rates are smaller for longer meridional waves and larger for shorter meridional waves.

\section{Instability radiation}

The unstable modes are either trapped around the mean jet or propagate energy away from the jet to affect the far region. Previous studies use certain criteria to determine the radiating modes. The criteria are based on the arguments that the eigenfunctions should look wavy for $k_{r}>k_{i}$ and the phase speed should lie within the allowable range for the barotropic Rossby wave phase speed $-\beta / 2 l^{2}<c_{r}<\beta / 2 l^{2}$. In the following, we examine these two conditions and discuss the energy flux.

Zonal structure: Figure 2-28 shows $\log \left(\left|k_{i} / k_{r}\right|\right)$ for the WBC (left) and EBC (right). The radiating modes reside at the longwave end for both the EBC and WBC. 
From the criteria that $\log \left(\left|k_{i} / k_{r}\right|\right)<0$, the WBC possesses more unstable radiating modes. As beta increases, shorter waves start to radiate for both the EBC and WBC, but the WBC supports radiating modes over a wider range in terms of the meridional wavenumber. This is different from what is found in Hristova et al. (2008) that the EBC supports a greater number of radiating modes over a wider range of meridional wavenumbers. This is a rather puzzling result. It is unclear why there is such a difference between results of the piecewise and continuous profiles over the longwave end. A direct comparison between this study with previous studies can be illuminating. It remains for a future study.
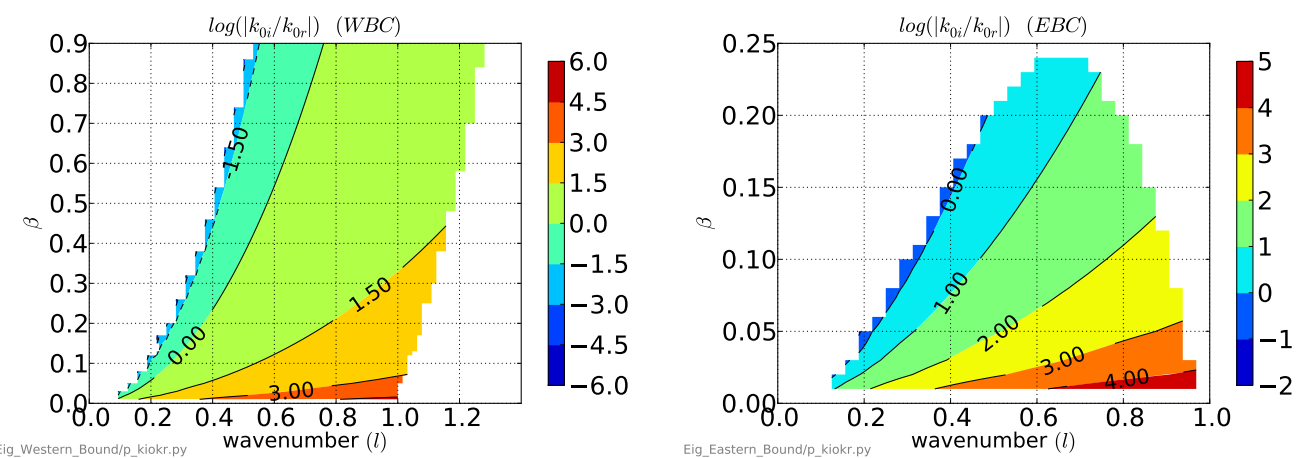

Figure 2-28: $\log \left(\left|k_{i} / k_{r}\right|\right)$ in the $(l, \beta)$ space for the EBC (right) and WBC (left). According to the criterion, modes with $\log \left(\left|k_{i} / k_{r}\right|\right)<0$ correspond to the wavylooking ones, which are more capable of radiating. Those modes have long meridional wavelength.

The phase speed condition The phase speed condition for the instability radiation states that in order for the unstable modes to radiate, the phase speed $c_{r}$ has to lie within the allowable range for the free Rossby wave phase speed. The range is $-\beta / 2 l^{2}<c_{r}<\beta / 2 l^{2}$. Here we have $c_{r}<0$, so we examine $c_{r}-\left(-\beta / 2 l^{2}\right)$, whose positive values mean the phase speed condition is satisfied.

Figure 2-29 shows the results, which are consistent with what is shown in Figure 228 that the long meridional waves are more capable of radiating. The WBC supports more unstable radiating modes than the EBC where very few unstable modes satisfy the phase speed condition. Again, this is different with the results in Hristova et al. 

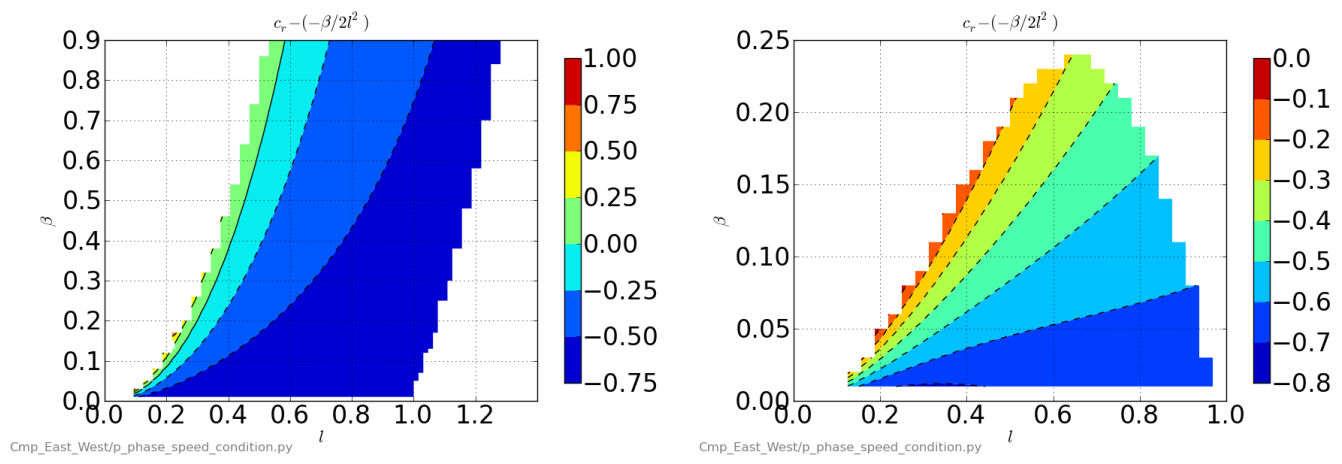

Figure 2-29: $c_{r}+\beta / 2 l^{2}$ for EBC (right) and WBC (left). The positive values correspond to modes that satisfy the phase speed condition for the instability radiation. Few modes for the EBC have positive values, and the zero line is barely observable. It is suspected that some of the marginally growing modes over the longwave end (with negligible growth rates) are not resolved. It remains a question for further investigations.

(2008). The discrepancy is also shown in the zonal wavenumber plots, but the reason remains unknown.

\section{Energetics}

The energy budget for the perturbations in a meridional jet is

$$
\frac{\partial}{\partial t} \frac{1}{2}|\nabla \psi|^{2}=-\nabla \cdot \tilde{S}+\psi_{x}^{*} \psi_{y} \bar{v}_{x}
$$

where

$$
\begin{aligned}
\tilde{S} & =\hat{i}\left[-\psi^{*} \psi_{x t}-\psi^{*} \bar{v} \psi_{x y}+\psi^{*} \psi_{y} \bar{v}_{x}-\frac{\beta}{2}|\psi|^{2}\right] \\
& +\hat{j}\left[-\psi^{*} \psi_{y t}-\psi^{*} \bar{v} \psi y y+\frac{\bar{v}}{2}|\nabla \psi|^{2}\right]
\end{aligned}
$$

For a bounded or trapped mode, the contribution from the flux divergence is zero over a region enclosing the unstable jet. For a radiating mode, however, the contribution from the flux divergence is non-zero over a closed region around the unstable region. The Reynolds stress transfers energy into the unstable mode, meanwhile the wave 
radiation drains energy away from the unstable region to slow down the growth. The contribution of the instability radiation to the energy budget resides in the flux term $S$, which is usually not important and omitted for the $f$ plane or the zonal jet problem.

Here we did not use the flux divergence terms to compute the radiated energy. Instead, we compute the radiated energy indirectly as it is computationally easier. The growth rate $c_{i}$ and the corresponding eigenfunction $\psi$ are known given the eigenvalue solution for an unstable mode. Define a box region $A$ over the unstable jet that extents the whole meridional region but a finite zonal distance, then the energy flux out of the box region by the radiation is

$$
E_{s}=<2 l c_{i} \frac{u^{2}+v^{2}}{2}>-<u v \bar{v}_{x}>
$$

where $<\cdot>$ means the average over the region $A$. The ratio of $E_{s}$ to the total energy input by the Reynolds stress $-\left\langle u v \bar{v}_{x}\right\rangle$ is defined as the index of the importance of the instability radiation.

Figure 2-30 shows the ratio for the EBC (red) and WBC (blue) with $\beta=0.05$. Long meridional waves tend to radiate more of their energy away both for the EBC and the WBC. This is consistent with previous conclusions based on the analysis of $\log \left(k_{r} / k_{r}\right)$ and the phase speed condition. $3 \%$ is not a significant number indicating the energy drain on the perturbation may be small, however, it could be a major source of energy for the distant interior circulation that has relatively weak local sources.

\subsection{Conclusion and discussion}

In this chapter, we study the barotropic instability of a meridional Bickley jet and focus on the influence of a side boundary, the influence of $\beta$ on an isolated meridional jet, and the influence of $\beta$ on the WBC and the EBC. 


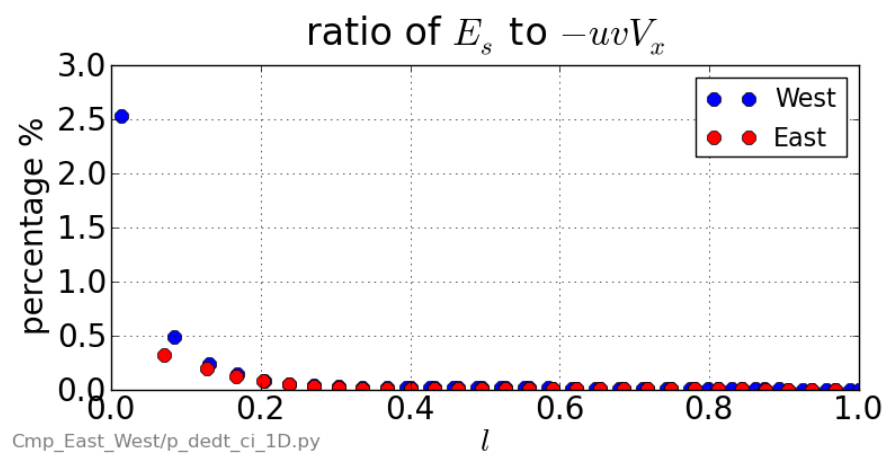

Figure 2-30: The ratios of $E_{s}$ and $\int_{x y}-u v V_{x}$. Red line for the EBC case and the blue line is for the WBC case. The long meridional waves are more likely to support the instability radiation.

The side boundary has a stabilizing effect on an unstable jet. The constraint of the side boundary reduces both the growth rates of the sinuous and varicose modes. However, if a side boundary is placed at the center of the jet, the sinuous mode disappears as it can not satisfy the boundary condition, but the varicose mode is left unaltered since it naturally satisfies the solid boundary condition.

For a meridional isolated jet, the growth rate of the varicose mode increases following the increase of beta, but the growth rate of the sinuous mode decreases. $\beta$ introduces zonal shifts in the unstable modes. The varicose mode and sinuous mode, which are orthogonal for an $f$ plane problem, start to project on each other because of the zonal shifts. The projection of the sinuous mode on the varicose mode increases the growth rate of the varicose mode, while the projection of the varicose mode on the sinuous mode decreases the growth rate of the sinuous mode.

Long meridional waves are more able to radiate. When an unstable mode is radiating, it has a smaller zonal wavelength at the eastern side of the jet, and a longer wavelength at the western side. This result is consistent with the result of Kamenkovich and Pedlosky (1996). Their cross-stream structure resembles the zonal structure in this study. The varicose mode is more able to radiate than the sinuous mode. When the sinuous mode is able to radiate, it only radiates westward. This feature, however, may depend on specific velocity profiles.

$\beta$ tends to stabilize the EBC but destabilize the WBC by changing the phase tilt. 
The tilts, however, cause different effects on the energy transfer from the mean to the perturbation. The tilts are more favorable for perturbations in the WBC to draw energy from the mean, but less favorable for those in the EBC. Consequently, the growth rate of the perturbation decreases in the EBC, but increases in the WBC. $\beta$ tends to raise the meridional phase speed of the perturbation in the WBC, but reduces that in the EBC. The different responses of the phase speed and frequency to the increase of beta bring an important asymmetry between the EBC and the WBC stability. Given the same frequencies, the zonal wavelength of the unstable mode in the EBC is larger than that in the WBC. This is consistent with the result of Hristova et al. (2008).

The study of this chapter is limited by the constraint of linear theory. It is left to a further nonlinear study (next chapter) to investigate to what extent the results of the linear theory hold, and to what extent the instability radiation is energetically influential for the interior of the domain. The energy change of the perturbation consists of two parts. One is the energy input from the mean, the other is the energy sink by the radiation. The energetics of barotropic instability is conventionally considered to be only related to the Reynolds stress, because the contribution of the flux divergence becomes zero for a bounded domain or trapped solutions. This is usually true for the zonal jet when the wave radiation in the meridional direction is negligible. For meridional jets, however, the beta effect may introduce significant energy radiation in the cross-stream direction and yield energy shedding away from the unstable region into the interior. The growth rate of the perturbation will be reduced by the energy loss to radiation. At the point that the energy radiation almost reaches the energy input, the growth rate becomes small, and the perturbation appears to be marginally unstable. Those modes are less important in the linear theory because they have small growth rates but could become competent agents for the energy radiation once they are energized by nonlinear interactions. We consider the nonlinear development of the radiating instabilities in the next chapter. 


\section{Chapter 3}

\section{Instability of an eastern boundary current: a nonlinear study}




\subsection{Introduction}

In order to fully understand instability radiation, nonlinear dynamics should be considered. As noted by McIntyre and Weissman (1978) and Kamenkovich and Pedlosky (1996), linear theory, by its nature, can not describe the radiation of strong instabilities. Strong spatial trapping for modes with large growth rates can be as temporary as the validity of the linear theory itself. In linear theory, perturbations are assumed to be weak and the exponential growth can be supported by eddies extracting energy from the mean. However, with exponential growth rates, unstable modes will soon gain finite amplitude, and consequently the nonlinear effects become important.

Furthermore, nonlinear effects can support energy transfer between modes, resulting in changes in growth rates and radiation characteristics of linear modes. Nonlinear interaction between two given sinusoidal waves with phases $\theta_{1}$ and $\theta_{2}$ can introduce new forcing terms to linearized equations with new phases $\theta_{1}+\theta_{2}$ and $\theta_{1}-\theta_{2}$. If at least one of these new phases match that of an intrinsic mode of the linear system, a resonance occurs in a similar way to that of a pendulum responding to an external forcing with a resonant frequency. As a result of the nonlinear resonance, transfer of energy takes place among a triad of waves. When the nonlinear energy transfer happens, a linearly neutral or decaying mode can become unstable and contribute to energy radiation. In fact, this is one of our main conclusions shown later.

In this chapter, a numerical model is used to study nonlinear effects on the radiating instability of an unstable eastern boundary current (EBC). We discuss the model setup and the methodology in section 3.2. Section 3.3 describes the processes of an unstable boundary current spinning up, becoming unstable, and generating finite amplitude perturbations and zonal jets. Based on these processes, we propose an hypothesis, which is later confirmed in section 3.4 where we use particularly designed numerical experiments to single out the nonlinear resonance process. Finally, section 6.3 provides the conclusion and discussion. 


\subsection{Methodology}

\subsubsection{Model}

As an extension of the previous linear stability study, this chapter is based on a barotropic QG model on a beta plane with nonlinear, forcing, and dissipation terms. The model is described by the barotropic vorticity equation,

$$
q_{t}+J(\psi, q+\beta y)=\mathcal{F}+\nabla \cdot A_{h} \nabla q
$$

where $q=\nabla^{2} \psi$ is the relative vorticity, $\psi$ is the streamfunction, $\beta$ represents the meridional gradient of the Coriolis parameter, $\mathcal{F}$ is the external forcing, $A_{h}$ is the lateral friction coefficient. We use the following scaling,

$$
\begin{aligned}
& t=\frac{t_{*}}{\beta L} ; x=L x_{*} ; y=L y_{*} ; \\
& \mathcal{F}=F g_{*}(x, y) ; u=\frac{F}{\beta} u_{*} ; \psi=\frac{F}{\beta} L \psi_{*} ; \\
& \epsilon=\frac{F}{\beta^{2} L^{2}} ; E=\frac{A_{h}}{\beta L^{3}}=\left(\frac{\delta_{M}}{L}\right)^{3} ; \delta_{M}=\left(\frac{A_{h}}{\beta}\right)^{1 / 3}
\end{aligned}
$$

where the asterisks denote the nondimensional variables, $L$ represents the width of the boundary current and $F$ is the scale of the forcing. $\delta_{M}$ denotes the length scale of the Munk layer. It is about $17 \mathrm{~km}$ along the eastern boundary in most cases here, smaller than the perturbation length scale which is in the order of the boundary current width $(50 \mathrm{~km})$. The vorticity equation can be nondimensionalized (after dropping asterisks ) as

$$
\nabla^{2} \psi_{t}+\epsilon J\left(\psi, \nabla^{2} \psi\right)+\psi_{x}=g(x, y)+E \nabla^{4} \psi
$$

where $\epsilon$ and $E$ measure the relative importance of the nonlinearity and the lateral friction. For a steady linear inviscid problem, the equation reduces to the Sverdrup relation $\psi_{x}=g(x, y)$. Although our numerical model is based on the dimensional form, we occasionally mention these nondimensional numbers. 
The vorticity equation can be rewritten in the flux form

$$
\nabla^{2} \psi_{t}+\nabla \cdot\left(\epsilon \mathbf{u} \nabla^{2} \psi+\psi \hat{x}+\mu \nabla^{3} \psi-E \nabla \psi\right)=f(x, y)
$$

A westward intensified lateral friction, as in Fox-Kemper (2003), is used to keep numerical stability, and also damp the unstable modes at the western boundary. Boundary conditions are the no-normal flow $(\psi=0)$ and slip conditions $(q=0)$.

The numerical realization is the same one as that used in the previous chapter. Details can be found in Appendix A.

\subsubsection{Energy budget}

In studies of the wave-mean flow interaction, the field is conventionally decomposed into a mean field and perturbations. The mean field is either represented by a spatial average or a temporal average. The spatial average is able to isolate the spatially independent component, such as the periodic jet. The temporal average can separate the steady component like steady gyres. In the second half of this thesis, the basic state contains a gyre structure, so the spatial (meridional) average becomes different from the temporal mean. In this chapter, a parallel meridional boundary current is used as the basic state. Theoretically, both the spatial and temporal averages render the same decomposition. Practically, however, the differences between those two decomposition methods could be large if the average is taken over a short period. We minimize the errors in the decomposition process by running the model for a sufficiently long period.

The total field then consists of two components

$$
\psi=\bar{\psi}+\psi^{\prime}
$$

where the overbar represents the time average. The Reynolds-averaged form of the 
barotropic vorticity equation is

$$
J(\bar{\psi}, \bar{q})+J\left(\overline{\psi^{\prime}, q^{\prime}}\right)+\beta \bar{\psi}_{x}=\mathcal{F}+\nabla \cdot A_{h} \nabla \bar{\psi}
$$

We henceforth denote the terms from left to right by 'mean', 'eddy', 'beta', 'forcing', and 'friction' respectively.

Subtracting the mean vorticity equation from the full equation gives the vorticity equation for perturbations

$$
q_{t}^{\prime}+J\left(\psi^{\prime}, \bar{q}\right)+J\left(\bar{\psi}, q^{\prime}\right)+J\left(\psi^{\prime}, q^{\prime}\right)-J\left(\overline{\psi^{\prime}, q^{\prime}}\right)+\beta \psi_{x}^{\prime}=\nabla \cdot A_{h} \nabla \psi^{\prime}
$$

With the perturbation being weak and the higher order terms neglected, equation 3.9 reduces to the one used in the linear theory (except for the friction term).

The energy budget for perturbations can be derived by multiplying $\psi^{\prime}$ on both sides of equation 3.9. After rearranging terms into flux forms, the time-averaged form of the perturbation energy equation becomes

$$
\begin{aligned}
\overline{\partial_{t} E^{\prime}} & +\nabla \cdot\left[\overline{-\psi^{\prime} \frac{D}{D t} \nabla \psi^{\prime}}+\overline{\overline{\mathbf{u}} E^{\prime}}+\overline{\mathbf{u}^{\prime} E^{\prime}}-\hat{x} \beta \overline{\psi^{2}}\right. \\
2 & \left.A_{h} \overline{\psi^{\prime} \nabla \nabla^{2} \psi^{\prime}}\right] \\
- & \nabla \cdot\left[\overline{\psi^{\prime}\left(\mathbf{u}^{\prime} \cdot \nabla\right)} \nabla \bar{\psi}+\overline{\psi^{\prime}(\overline{\mathbf{u}} \cdot \nabla) \nabla \psi^{\prime}}\right] \\
& =-\left(\overline{\left.\mathbf{u}^{\prime} \mathbf{u}^{\prime} \cdot \nabla\right)} \cdot \overline{\mathbf{u}}+A_{h} \overline{\nabla \psi^{\prime} \cdot \nabla \nabla^{2} \psi^{\prime}}\right.
\end{aligned}
$$

where $E^{\prime}=\frac{1}{2}\left|\nabla \psi^{\prime}\right|^{2}, \mathbf{u}=\hat{z} \times \nabla \psi$, the overbar represents the time mean (notice that $\left.\overline{E^{\prime}} \neq 0\right)$, and $D / D t=\partial / \partial_{t}+\mathbf{u}^{\prime} \cdot \nabla$.

The first term on the left-hand-side (lhs) vanishes for an equilibrium state. Among the flux terms, $\overline{-\psi^{\prime} \frac{D}{D t} \nabla \psi^{\prime}}$ represents the pressure work (Pedlosky, 1987), $\overline{\overline{\mathbf{u}} E^{\prime}}$ and $\overline{\mathbf{u}^{\prime} E^{\prime}}$ are related to the advection of the perturbation energy by the mean and perturbation itself, and the remaining two terms are the beta flux and the smoothing effect of the lateral friction. The flux terms only redistribute energy and have zero net energy contribution in a closed domain. 
The two terms on the right-hand-side (rhs) are the energy transfer by the Reynolds stress (the first term) and the frictional dissipation (the second term). For a domain with periodic or no-flux boundary conditions, the integrated energy budget is simply the balance between these two terms. It can be shown that the same Reynolds stress terms (but with opposite sign) exist in the energy budget for the mean state. It is obvious that without forcing and dissipation, energy is transfered between the mean and the perturbation fields through Reynolds stress, and the total energy is conserved over the whole basin.

For a zonally parallel flow, the Reynolds stress reduces to $-u^{\prime} v^{\prime} \partial_{y} \bar{u}$, meaning that eddies gain energy from the mean if the perturbation streamlines lean against the mean shear. Similarly, for a meridionally parallel flow, the terms reduce to $-u^{\prime} v^{\prime} \partial_{x} \bar{v}$.

\subsubsection{Forcing}

We will study the instability of a forced boundary current. The basic state with a boundary current is the main driver of the growth of perturbations. In linear theory, we assume the basic state is somehow maintained by an external forcing, and is steady. There are two ways to set up the basic state in the numerical model. One way is to directly specify a fixed boundary current in the model. The other way is to form a boundary current by specifying an external forcing. The difference between these two methods is that lateral friction affects both the perturbation and the boundary current in the forced case but affects only perturbation field in the case with a specified boundary current. We use the external forcing method in a qualitative study of the spin-up process in section 3.3, and use a fixed boundary current for the mechanism study in section 3.4 .

The forcing field is calculated according to the Sverdrup relation, which states that in an inviscid and steady flow the vortex stretching or squashing by the Ekman pumping is balanced by the meridional advection of the planetary vorticity

$$
\beta \bar{v}=\mathcal{F}
$$


where $\mathcal{F}$ is a body force that is related to the layer depth $D$, the Coriolis parameter $f$ and the Ekman pumping velocity $w_{e}: \mathcal{F}=f w_{e} / D$. For a boundary current $\bar{v}=$ $-\bar{V} \operatorname{sech}^{2}\left[\left(x-x_{c}\right) / L\right]$, where $\bar{V}$ represents the scale of the boundary current velocity, $x_{c}$ represents the zonal location of the center of the jet and $L$ represents the width of the jet, the corresponding external forcing is

$$
\mathcal{F}=\beta \bar{v}=-\beta \bar{V} \operatorname{sech}^{2}\left(\frac{x-x_{c}}{L}\right) .
$$

Then the scale of the forcing is $F=\beta \bar{V}$, and the nonlinearity parameter based on the width of the $\mathrm{EBC}$ is $\epsilon=F / \beta^{2} L^{2}=\bar{V} / \beta L^{2}$. Recall that in the linear problem, we defined the nondimensional beta as $\beta^{*}=\beta L^{2} / \bar{V}$, so that the nonlinearity of the new problem is the inverse of the nondimensional beta in the linear problem.

However, there are friction and eddies in the numerical experiments. The final boundary current in the numerical simulation will have a different structure from the one calculated according to the Sverdrup relation. The boundary current will be distorted by strong nonlinearities and friction.

Lateral friction broadens the boundary current in the cross-stream direction. An example with a weak forcing shows the frictional effect on the forced boundary current. Figure 3-1 shows zonal structure of the streamfunction in the case with $\bar{V}=0.05 \mathrm{~m} / \mathrm{s}$, $\beta=2 \times 10^{-11} / \mathrm{ms}$, which is a typical mid-latitude value, and $A_{h}=100 \mathrm{~m}^{2} / \mathrm{s}$ along the eastern boundary, after the system reaches equilibrium. These parameters yield a Reynolds number $\left(V L / A_{h}\right)$ of 25 for a $50 \mathrm{~km}$-wide boundary current. In this example, the boundary current is parallel in $y$ with no significant perturbations, and the effect of perturbations is negligible. The streamfunction structure along the western boundary shows a Munk layer solution. The Munk layer width according to the scale $\delta_{M}=$ $\left(A_{h} / \beta\right)^{1 / 3}$ is about $80 \mathrm{~km}$, which is consistent with the figure.

Figure 3-2 shows the $y$-averaged zonal structures of the along-boundary velocity in experiments with different friction coefficients but without eddies. It shows that EBCs are of similar structures to the forcing, except that the jet centers shift eastward 


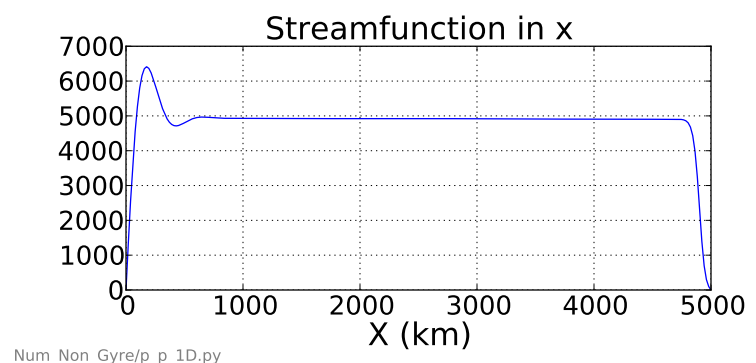

Figure 3-1: The zonal structure of the streamfunction in the experiment with this forcing $F=\beta \bar{V} \operatorname{sech}^{2}\left(\frac{x-x_{c}}{L}\right)$, where $\bar{V}=0.05 \mathrm{~m} / \mathrm{s}, \beta=2 \times 10^{-11} / \mathrm{ms}, x_{c}=4900 \mathrm{~km}$, and $L=50 \mathrm{~km}$. The final state is steady. The boundary current does not develop significant unstable perturbations.

once the lateral friction is included. The eastward shift is intensified with a larger friction coefficient. Notice that the eastward shift of the jet center is not a general phenomenon. It is caused by the particular jet structure $(\operatorname{sech}(x))$ and the particular form of the lateral friction $\left(\nabla^{4} \psi\right)$. The zonal shift may be different for different velocity profiles.
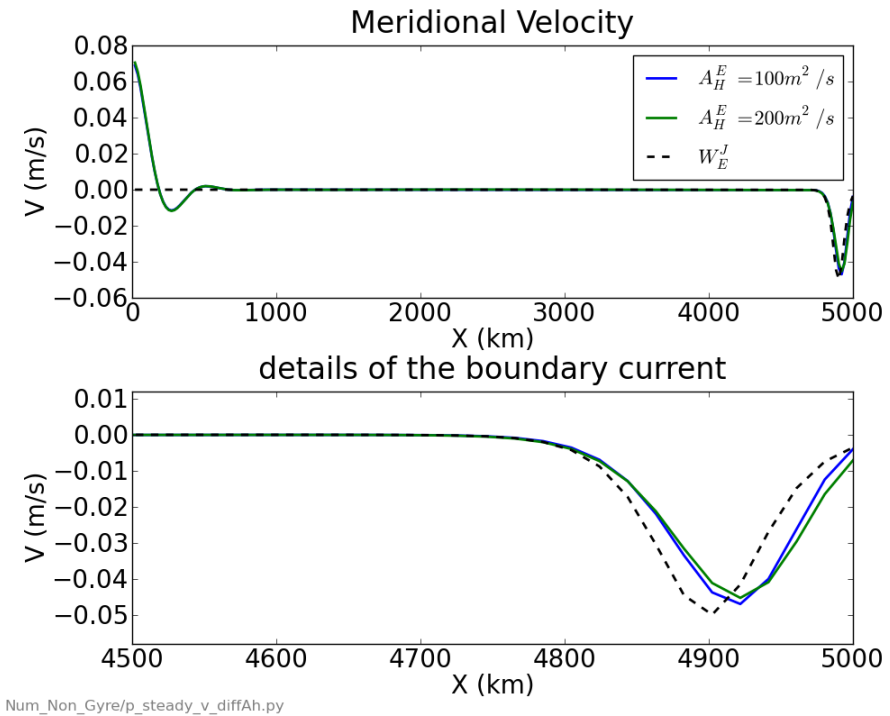

Figure 3-2: The zonal structure of the meridional velocity in the case with negligible developed perturbations. The black dashed lines shows the profile of the prescribed forcing $\mathcal{F} / \beta$. The blue and green lines show the final boundary current structures in two cases with different diffusivity. 

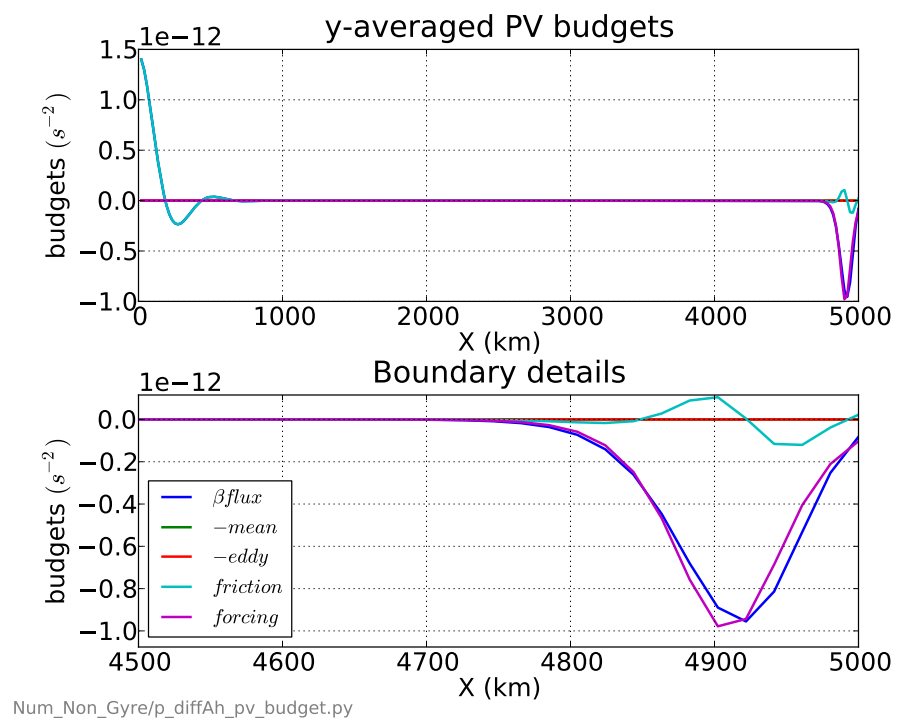

Figure 3-3: PV budget in the case without significant perturbations. The balance is among the forcing, the beta flux, and the lateral frictional flux.

Figure 3-3 shows the initial PV budget in equation 3.8. In this case, the growth rates of perturbations are very small because of the weak forcing. The amplitude of the initially-specified random noise is still small (5 orders of magnitude smaller than the mean), the perturbation PV flux divergence is negligible. The PV budget along the eastern boundary is balanced by three terms, the mean, frictional, and beta fluxes, among which the forcing and beta flux dominate. The frictional term redistributes PV across the jet, shifting the jet center eastward.

We could have derived the forcing field for a perturbation-free state by directly solving the following steady linear PV equation while including lateral friction for a given boundary current profile:

$$
g(x)=\beta \bar{v}-A_{h} \bar{v}_{x x x}
$$

However, we tested and compared simulations using both methods, and find they produce the same qualitative results. The first method, in which the forcing is calculated without friction, is used in section 3.3, where a qualitative description of the spin-up process is studied. In section 3.4, where detailed mechanisms are studied, we use a 
fixed boundary current to better predict the initial growth rates of different modes in a frictional system.

\subsection{The spin-up process}

In this section, we observe and study how a boundary current spins up and becomes unstable, and how perturbations in the boundary current grow and feed back on the boundary current. By observing the process, we gain a first impression of the nonlinear dynamics of an unstable EBC.

As discussed in section 3.2.3, the EBC is maintained by an external forcing field $\mathcal{F}$. The forcing is set up following equation 3.12 with $L=50 \mathrm{~km}, x_{c}=4900 \mathrm{~km}$, where $x_{c}$ is the location of the center of the boundary jet. The model has a north-south periodic condition. Two experiments with different forcing strengths are conducted. The first experiment with $F=3 \times 10^{-12} s^{-2}$ is denoted by $C 1$ and the second one with $F=4 \times 10^{-12} s^{-2}$ is denoted by $C 2$. The two forcing strengths correspond to boundary current velocity scales $0.15 \mathrm{~m} / \mathrm{s}$ in $C 1$ and $0.2 \mathrm{~m} / \mathrm{s}$ in $C 2$.

Figure 3-4 shows the spin-up process in terms of the streamfunction. After the surface forcing is turned on, the streamfunction increases within the eastern boundary region in response to the forcing field. Signals propagate westward. A western boundary current is generated once the signal from the eastern boundary reaches the western boundary. This is a rather fast process in this barotropic case. The basic structure of the boundary current is established in 30 days. The wavy signal is gradually damped out after the eastern boundary current reaches a steady state, in which the vorticity input by the external forcing is balanced by the beta and frictional fluxes. The final steady state consists of a forced eastern boundary current, a quiescent interior, and a frictional western boundary current.

Small random noises introduced to the established unstable EBC will grow. Figure 3-5 descriptively shows the evolution of perturbations in $C 1($ where $\bar{V}=0.15 \mathrm{~m} / \mathrm{s})$. At an early stage $t=T_{0}$, perturbations are self-organized, showing an unstable normal 


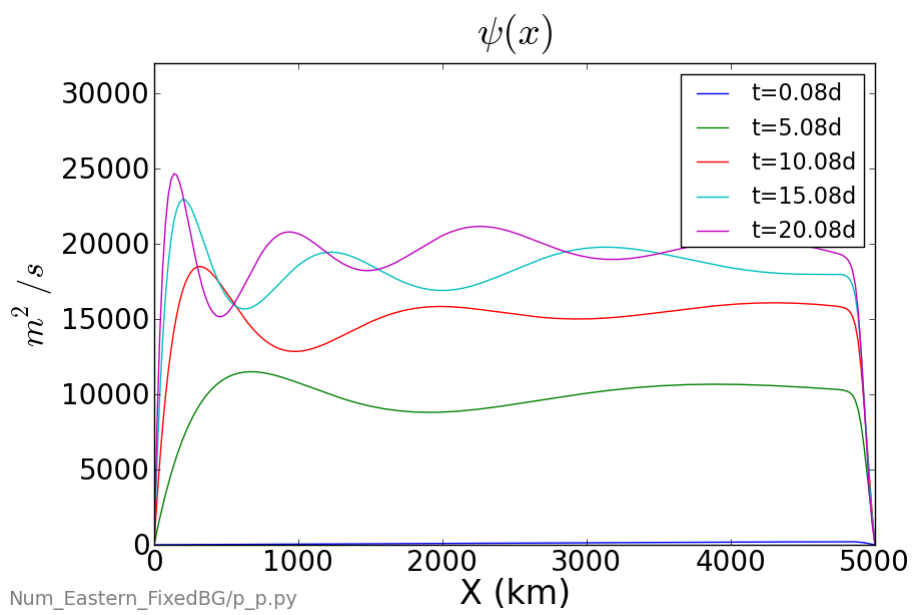

Figure 3-4: The zonal structure of the streamfunction at different stages. After the forcing is applied along the eastern boundary, a large zonal gradient forms to support the boundary current. Meanwhile, the lifted streamfunction (pressure) propagates westward. The western boundary current is formed when the streamfunction is reduced to zero from the interior value. The model is periodic in the north-south direction. The boundary condition $\psi=0$ is satisfied at both boundaries, requiring $\int V d x=0$, which is confirmed in the model.

mode structure. At $t=T_{1}$, the nonlinear interaction becomes intense, producing a new structure with larger zonal and meridional scales in the north-eastern corner. Nonlinear interaction becomes more prominent later at $t=T_{2}$, and the perturbation field extends westward into the basin interior. At $t=T_{3}$, the system reaches an equilibrium with a well-organized perturbation field. The total kinetic energy of the system increases during this process.

The increase of perturbation activity also affects the mean current. As shown in the bottom panel of Figure 3-5, the boundary current is weakened and broadened, which is what typically happens in the case of an unstable barotropic jet. In the PV budget shown in Figure 3-6, we see that the effect of perturbations is similar to the effect of lateral friction, which redistributes PV across the jet, resulting in a broadened and weakened mean current.

Phenomenologically, the spin-up process can be broken up into the following steps. The EBC is formed quickly after the forcing is turned on. Once the EBC forms, the 

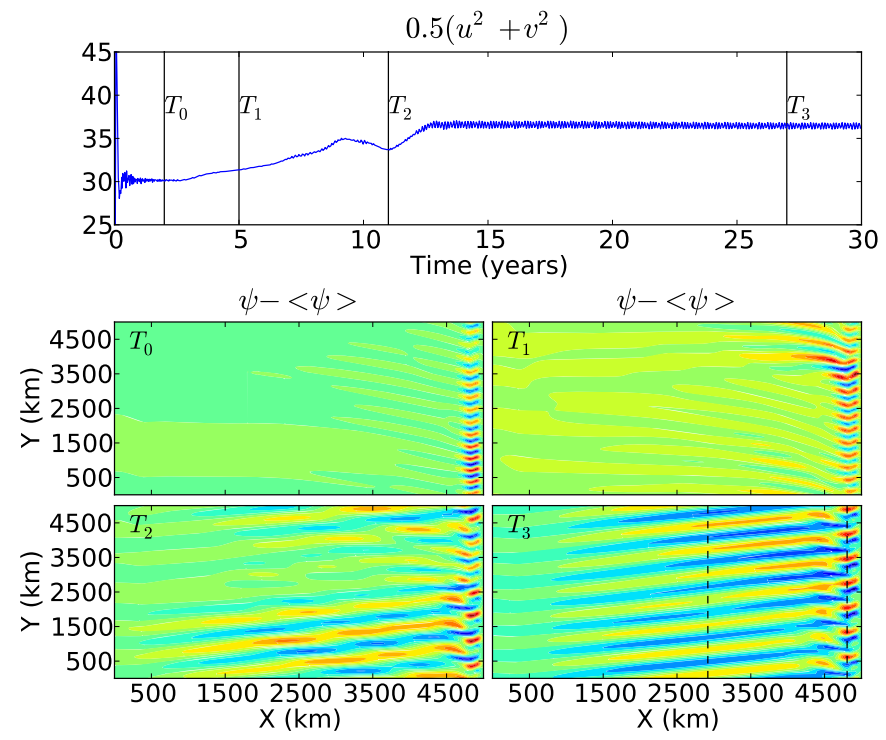

mean meridional velocity

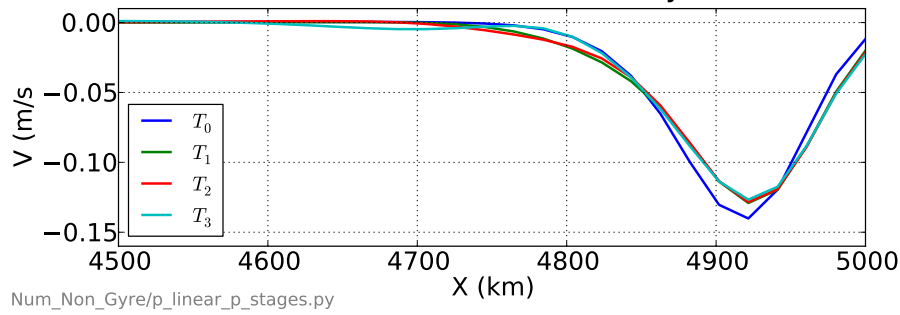

Figure 3-5: The upper panel shows the total kinetic energy as a function of time. The middle 4 panels show 4 snapshots at $t=T_{0}, T_{1}, T_{2}, T_{3}$ indicated by the vertical lines in the upper panel. The bottom panel shows the zonal structures of the y-averaged meridional velocity for the four snapshots.

most unstable mode stands out quickly from the initial random noise during the early stage. If the growth rate of the most unstable mode is much larger than the rest of the modes, the pattern of the most unstable mode will be easily detected. During the later stage, other modes appear either because of their own unstable character or nonlinear interactions. The well-organized patterns in this particular experiment imply that the nonlinear interaction may be weak and only exist among a limited number of modes. In order to further distinguish the mechanism that governs the dynamics during the later stage, a Fourier analysis in the streamwise direction is conducted to scrutinize the characteristics of each normal mode.

The total field in a parallel flow problem can be represented by Fourier modes in 


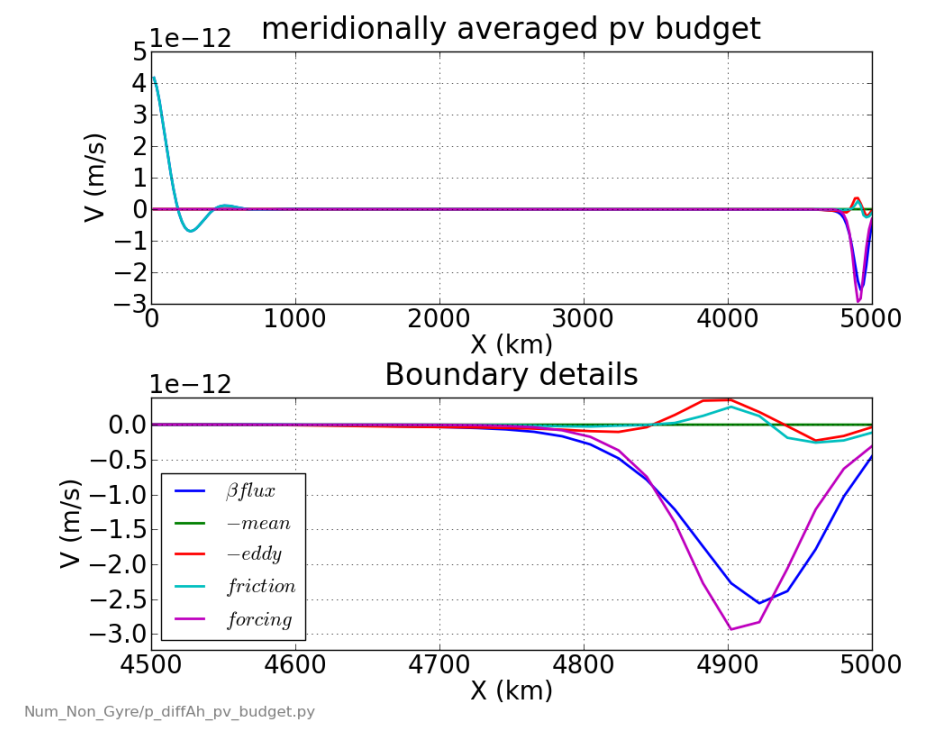

Figure 3-6: PV budget for $C 1$. The eddy flux and the frictional flux have similar weakening and broadening influences on the mean current.

the streamwise direction. The decomposition in a continuous form is

$$
\hat{\psi}\left(\ell ; x_{s}, t_{s}\right)=\int_{-W_{y} / 2}^{W_{y} / 2} \psi\left(y ; x_{s}, t_{s}\right) e^{-i 2 \pi \ell y} \mathrm{~d} y .
$$

where $x_{s}$ and $t_{s}$ are constants in each decomposition, meaning that for each snapshot at $t=t_{s}$, we conduct one dimensional Fourier analysis at $x_{s}$ along $y$ to evaluate the meridional wavenumber spectra, $\hat{\psi}\left(\ell ; x_{s}, t_{s}\right)$.

Figure 3-7 shows the Hovmöller diagram of the wavenumber spectra at two zonal locations marked by the two dashed lines in the $T_{3}$ panel of Figure 3-5, one in the middle of the zonal domain span (the upper panel) and the other in the EBC region (the lower panel). In the EBC region, the most unstable mode stands out with a meridional wavelength $357 \mathrm{~km}(l=0.0028$ denoted by M2) at about year 2.5. This mode dominates until year 7, when modes with longer and shorter meridional wavelength emerge. The longer wave with a wavelength $714 k m(l=0.0014$ denoted by $M 1$ ) is the subharmonic of the most unstable mode.

In the middle of the domain, there is only one dominant mode, M1. It simulta- 


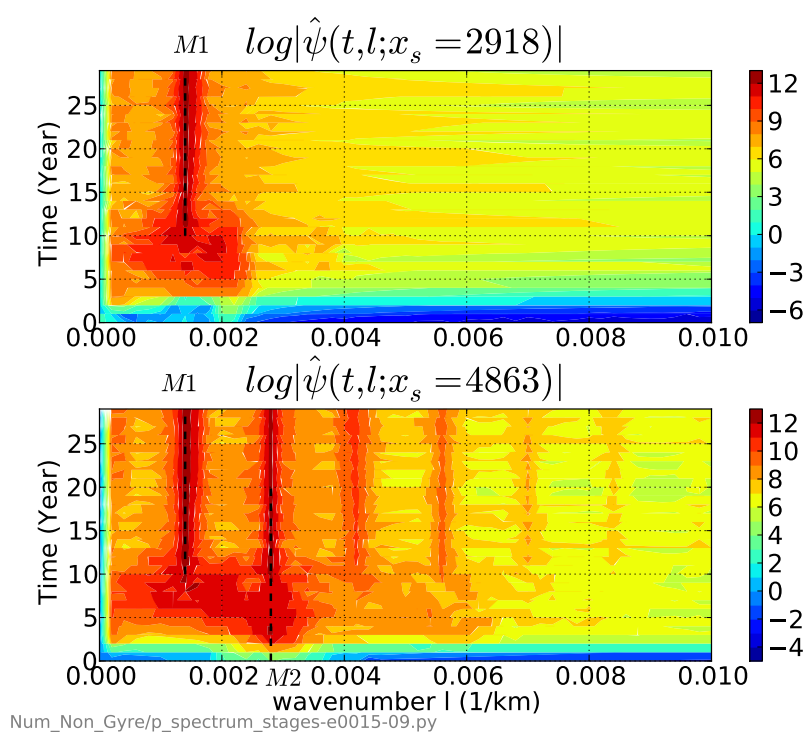

Figure 3-7: The Hovmöller diagram of the meridional wavenumber spectrum of the streamfunction at two zonal locations, the boundary region (lower panel) and the interior (upper panel).

neously appears both in the EBC region and in the interior (compare the timing of this mode in the lower panel with that in the upper panel). Given the fact that the energy source is always at the EBC region, the concurrence of this mode in the EBC and interior means that its zonal propagation is a quick process.

Two modes dominate energetically, but only the longer mode $M 1$ prevails in the interior. This is because of their different radiating abilities. As shown in Figure 3-8, the longwave mode $M 1$ has long tails to the west, indicating it is more able to radiate, while shortwave mode $M 2$ is mostly trapped within the EBC. This is consistent with the result of the linear stability theory in the previous chapter, i.e., modes with longer meridional wavelengths are more able to radiate.

An analysis of the power spectra indicates that the long radiating mode is supported by subharmonic instability, which is one particular case of triad-resonance. In this case, two of the triad waves have the same frequency and wavenumber, but may have different eigen-structures. Subsequently, a series of modes, which satisfy the phase relation of a triad with the two harmonics, can be generated through nonlinear 

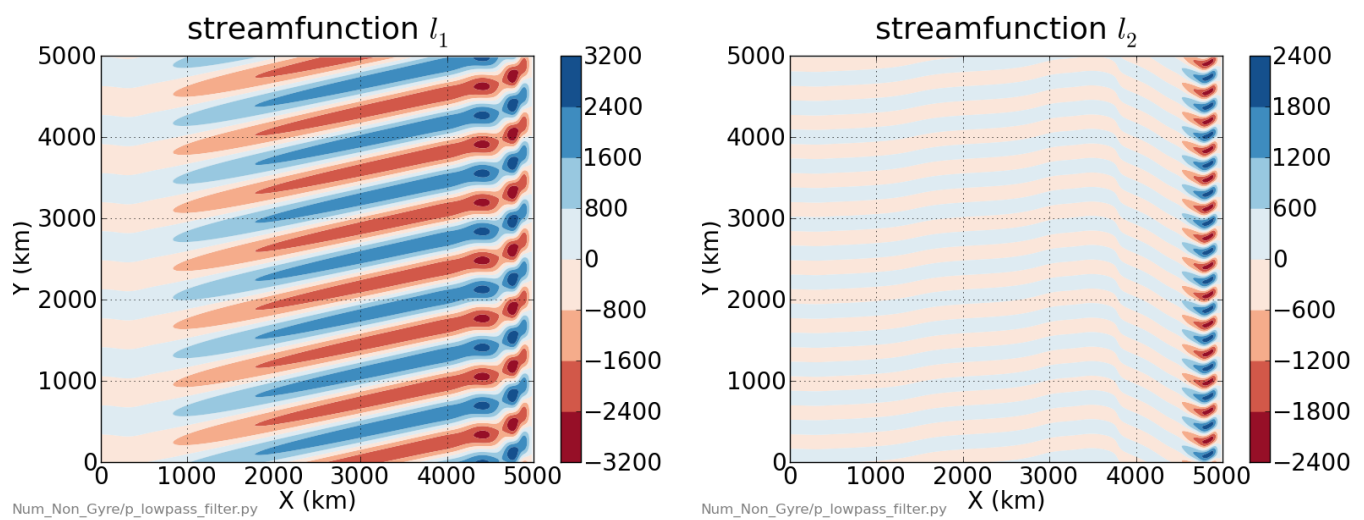

Figure 3-8: Streamfunction reconstructions for the $M 1$ and M2 using Fourier transform.

energy transfer. However, resonance does not occur for modes that are not natural ones of the system. As a result, these modes are significantly weaker. This argument can be confirmed by an example of the wavenumber spectrum in the EBC region at year 30, which is shown in Figure 3-9. The energy peaks at a series of harmonics and decays following the increase of wavenumber.

Based on all the evidence, we propose the following hypothesis in terms of the energy route for an unstable EBC. External forcing inputs energy into the boundary current, which becomes unstable and further transfers energy into the perturbation field through the Reynolds stress. Part of the perturbation energy is dissipated locally; the remaining energy is radiated into the interior, where the energy brought by eddies (or waves) is dissipated by lateral friction. Later, the energy transfer reaches balance and the system reaches equilibrium. However, one question still remains. Is the radiating wave (M1) one of the unstable modes of the boundary current, or merely supported by nonlinear interactions?

Two experiments are carried out to implicitly answer the question, and will discuss detailed mechanisms in 3.4. In the first experiment, all modes are filtered out except for the mean current and $M 1$ at each time step of the model integration. The hypothesis is that $M 1$ will have finite amplitude if it is one of the unstable modes of the mean current. If this is the case, then our above argument fails. If $M 1$ is not 


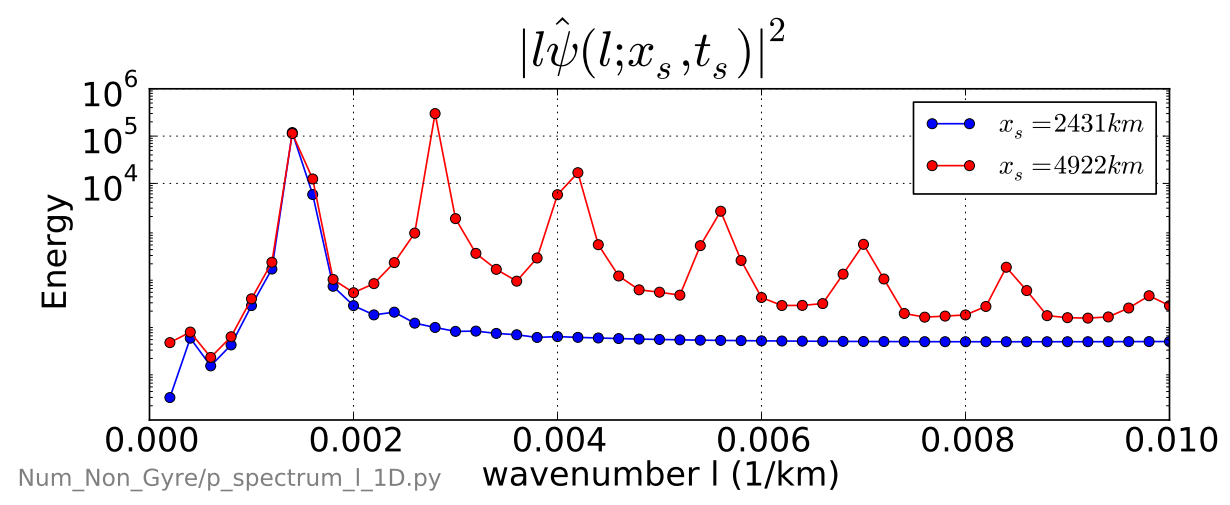

Figure 3-9: One-dimensional power spectra density in terms of wavenumber in the EBC region (the red line) and the interior (the blue line) at the end of the year 30 . The power density is calculated as $|l \hat{\psi}|^{2}$, which corresponds to $u^{2}$ in physical space. The spectra density of the total kinetic energy, $\left(u^{2}+v^{2}\right) / 2$, has a similar structure, which is not repeated here. In the EBC region, the power density peaks at $l=n \times 7 \ell$ with $\ell=2 \pi / W_{y}$ being the smallest wavenumber resolved by the model and $n=1 \cdots N_{y} / 2$, where $N_{y}$ represents the number of grid points in $y$. In the interior (the blue line), the kinetic energy mainly reside in $M 1$. The match of the blue and red lines at the longwave end indicates that these longwave modes have very small westward decay rates.

sustained, then we continue the second experiment, in which M2 is added back to the model. The verification process is straightforward. M1 is supported by nonlinear interactions if it is sustained by adding M2. In both experiments, the model starts with an equilibrium state and is run for three years. Results are shown in Figure $3-10$.

In the first experiment (red lines), energy of $M 1$ (red dashed line) continuously declines, which means that $M 1$ is not an unstable mode for the mean. In the second experiment, to the contrary, energy is sustained at about the same level for all three components (the mean, M1, and M2) which means that major energy transfers take place among them, and that M1 is supported by M2. It is worth noticing in the first experiment that when $M 1$ weakens because of lack of the $M 2$ support, the energy of the mean (red solid line) increases, indicating that the mean EBC regains energy when perturbations and the associated Reynolds stress diminish.

The energy Hovmöller diagrams of the two modes are shown in Figure 3-11. Again, 


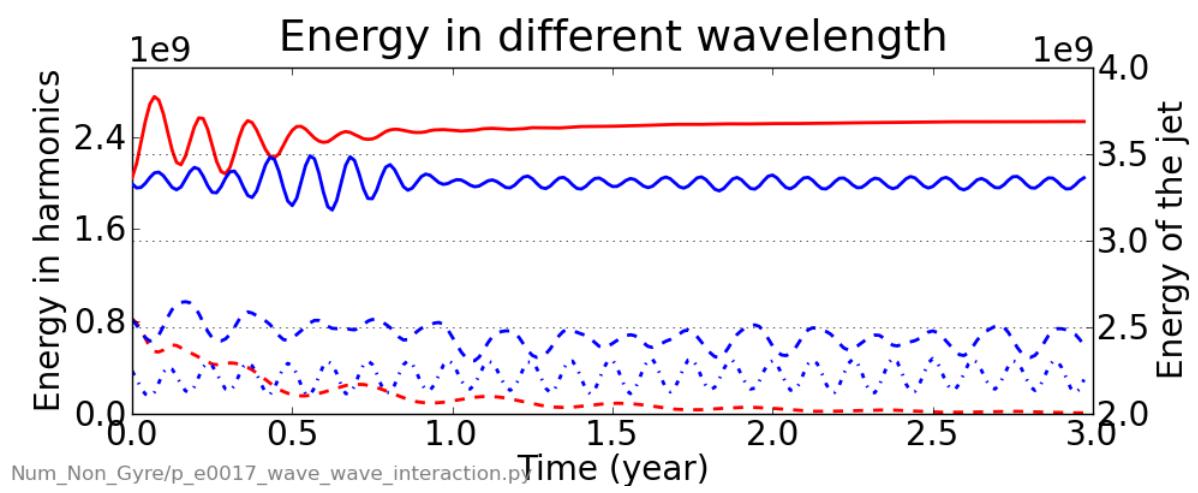

Figure 3-10: The kinetic energy evolution of the mean (solid lines, right y-axis), M1 (dashed lines), and M2 (dotted line). Red lines represent the case with only the EBC and M1. Blue lines represent the case with the EBC, M1, and M2. It is clear that M1 is not sustained by the EBC but by the M2. Refer to the text for details.

the westward decay rate of the energy is smaller for $M 1$ than that for $M 2$. The perturbation energy of $M^{2}$ is mostly trapped near the EBC.
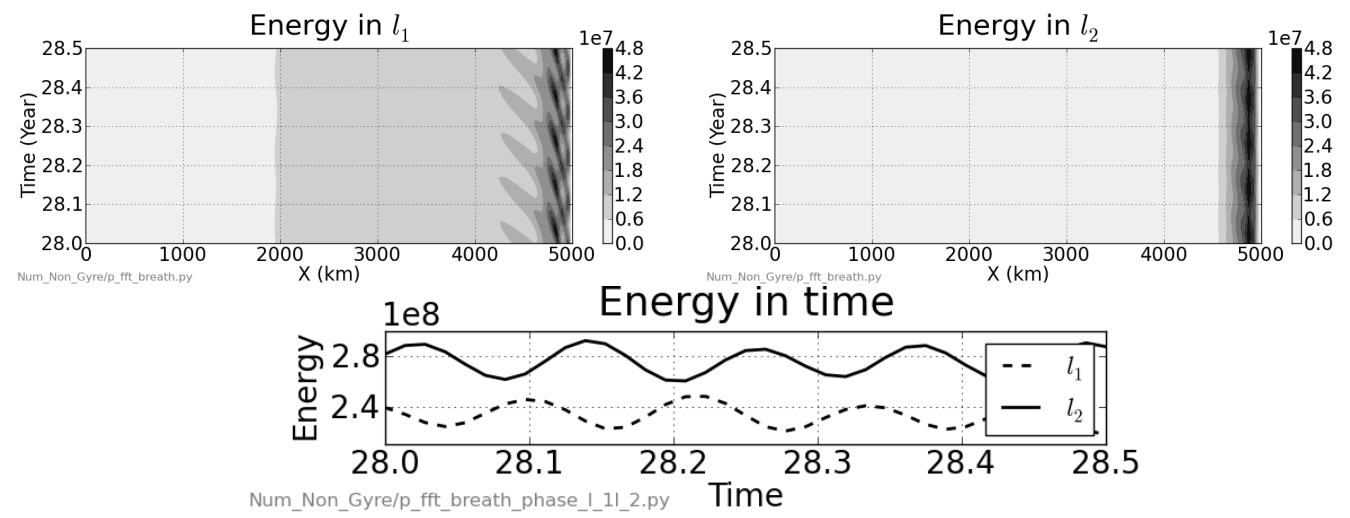

Figure 3-11: The kinetic energy Hovmöller diagrams for $M 1$ (upper left) and M2 (upper right). The $y$-integration is taken to calculate the kinetic energy, $E(x, t)=$ $\int_{0}^{W_{y}} 0.5\left(u^{2}+v^{2}\right) \mathrm{d} y$. The lower panel shows the time evolution of the energy integrated within a boundary region, $\int_{4800 k m}^{5000 k m} E(x, t) \mathrm{d} x$.

The lower panel in Figure 3-11 shows the time series of the energy of $M 1$ and M2 integrated over the boundary region. There is an interesting "breathing" feature. Even during equilibrium, the energy in different modes oscillates in time. They have a phase shift approximately $\pi$. When $M 2$ is in the energy peak, $M 1$ is in the energy trough. This is further evidence of the energy transfer between these two modes. 
First, the M2 mode gains energy from the basic state. The subharmonic mode $M 1$ is generated after $M 2$ obtains a finite amplitude and nonlinear interactions become important. M2 experiences an energy trough as it passes energy to $M 1$, while $M 1$ experiences the opposite. Notice that the summation of the energy in the two mode is not constant, meaning that these two modes also interact with other modes.

In this experiment, denoted by $C 1$, the forcing is weak. As a result, only several discrete modes prevail. In the next experiment, denoted by $C 2$, we increase the forcing strength and correspondingly the nonlinearity. Stronger nonlinear interactions and more efficient energy redistributions among modes are expected.

Figure 3-12 shows the same fields as in Figure 3-5 but for C2. Different from that in $C 1$, the total energy exhibits strong variability (upper panel). The narrow zonal jets extend into the far west, but have no well-organized patterns at the end of the simulations (refer to $t=T_{3}$ panel). The EBC is significantly broadened and weakened (lower panel), and shows a secondary meridional current formed around $x=4650 \mathrm{~km}$ (refer to the cyan line in the lower panel). Comparing the meridional mean structure of the EBC at $T_{3}$ with the one at $T_{2}$, we find that, with a strong nonlinearity, the meridional mean of the boundary current is no longer fixed in time. The time variation of the meridional mean is caused by the time variation of the perturbation field evident in the lower panel in Figure 3-11.

The perturbation PV flux becomes larger in a system with a stronger forcing. Figure 3-13 shows the PV budget in C2. The perturbation flux is similar to the one in $C 1$ (Figure 3-6) but with a stronger amplitude and a wider zonal span. Eddies transport PV farther off the boundary to form a secondary jet shown in the lower panel of Figure 3-12. As a result, the boundary jet becomes wider and weaker.

The wavenumber spectrum in Figure 3-14 shows that no distinct harmonics are present in C2. The spectrum is broader and more continuous in both the EBC and interior, indicating stronger nonlinear interactions. However, it is still true that only 

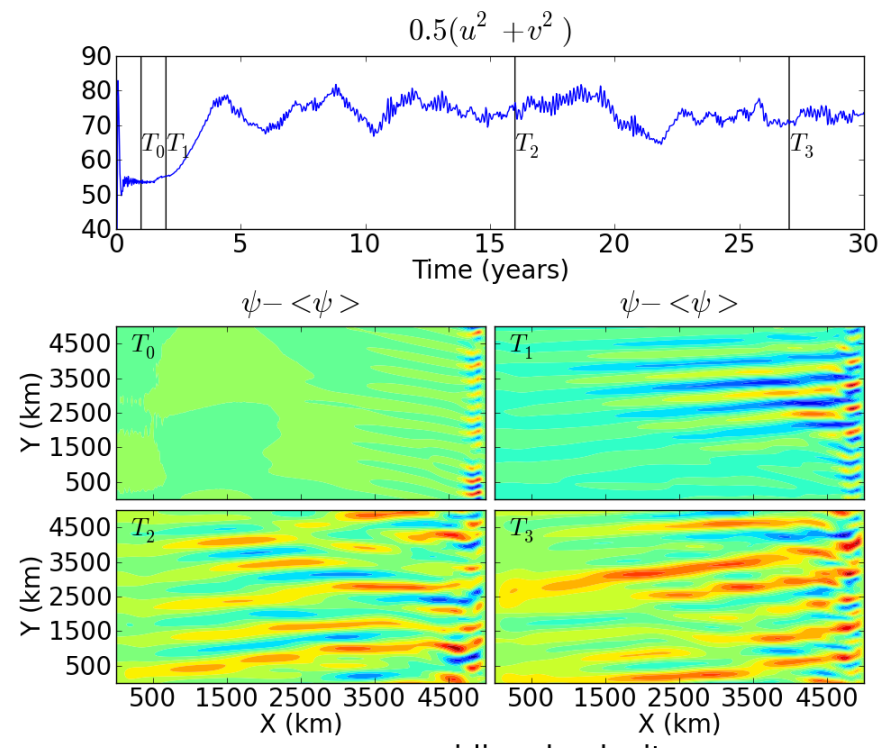

mean meridional velocity

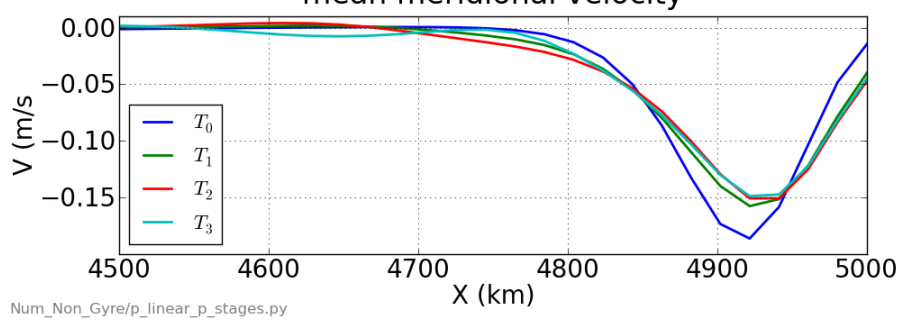

Figure 3-12: The same as Figure 3-5 but for $C 2$ with a stronger forcing. The forcing scale of the vorticity equation is $F=4 \times 10^{-12} \mathrm{~s}^{-2}$, corresponds to $\bar{V}=0.2 \mathrm{~m} / \mathrm{s}$.

the longer meridional wave can radiate. The energy of short waves in the interior is negligible.

We compare the zonal energy structure of the perturbation and mean in the two experiments, C1 and C2. Results are shown in Figure 3-15. Energy is scaled in such a way that $\iint E_{\text {jet }}(x, y) \mathrm{d} x \mathrm{~d} y=1$. Again, we see the relative strength of the boundary current is weaker and broader in C2 by comparing the solid lines. The perturbation kinetic energy, relative to the mean energy, is stronger and decays slower in C2 than that in $C 1$ (dashed lines). The interior is remotely forced by the unstable boundary current through the radiating instabilities.

Up to now, we have discussed the evolution of a forced unstable EBC and the associated perturbation field. We summarize/hypothesize the spin-up processes in 


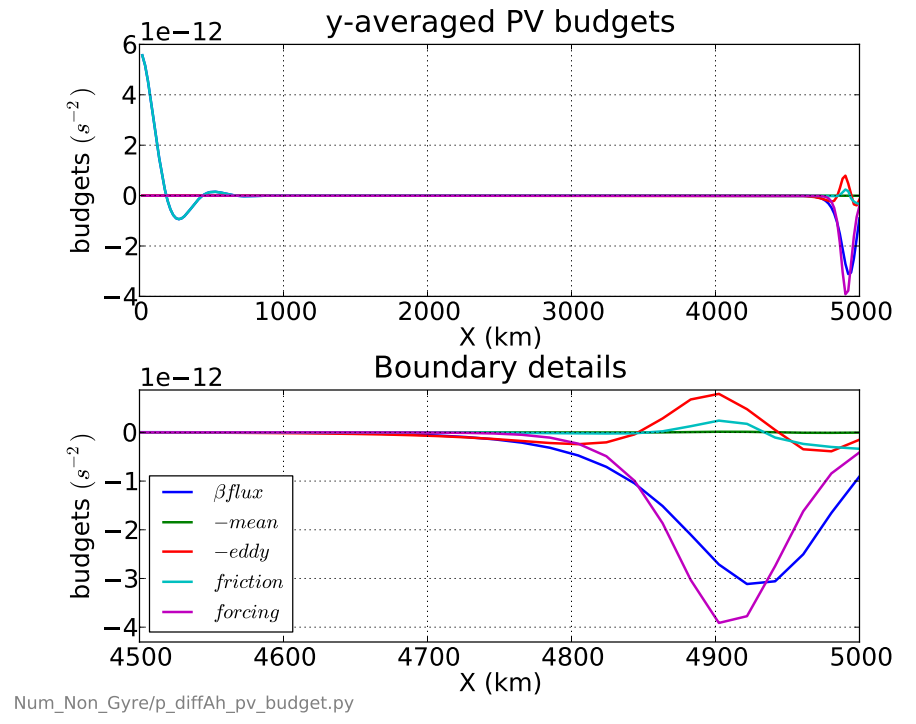

Figure 3-13: PV budget for $C 2$ with $F=4 \times 10^{-12} s^{-2}$.

those experiments as following.

1. After the forcing is turned on, the EBC is formed quickly in the order of a month. A WBC can be excited depending on the boundary condition. If the western boundary condition is the same as the eastern boundary condition, i.e., $\psi=0$, a western boundary layer, which is similar to the Munk layer, is formed. The consistency of the boundary condition between the eastern boundary and the western boundary implicitly assumes the mass exchange between two boundaries. One can think the southwest corner is connected to the southeast corner, and also the northwest and northeast ones. However, if the $\psi$ at the western boundary is allowed to match the interior value, no pressure gradient can form there, so that no western boundary layer/current is generated.

2. Perturbations start to grow in the form of the most unstable mode after the EBC is established.

3. The most unstable mode has a small meridional wavelength and is non-radiating. It continuously accumulates energy to gain a larger amplitude.

4. The subharmonic of the most unstable mode appears at a later stage because 


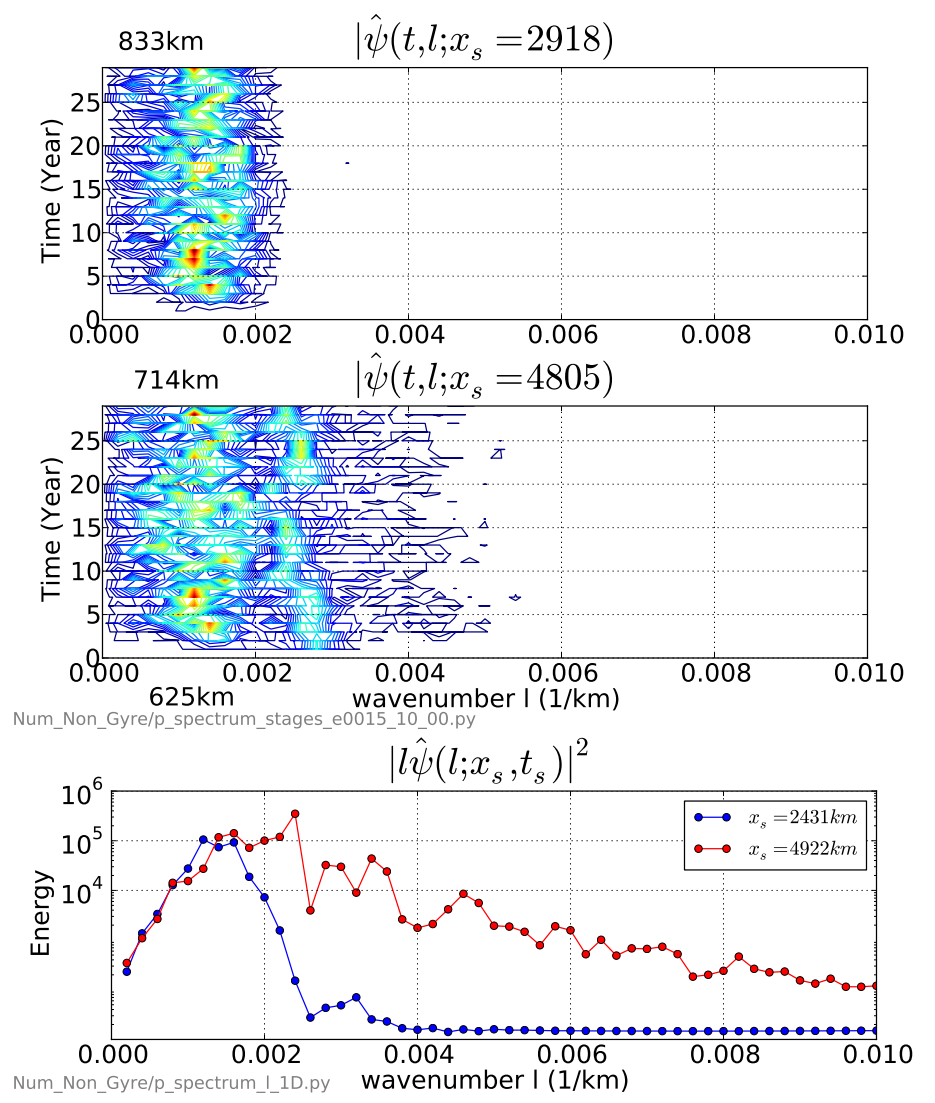

Figure 3-14: One-dimensional wavenumber spectra for C2. The upper two panels are the Hovmöller diagrams of the power density, the same as Figure 3-7 but for C2 . The lower panel is the C2 version of Figure 3-9. The spectrum is more continuous because the stronger nonlinear interactions more effectively spread energy among different modes.

of the subharmonic instability. The most unstable mode transfers energy to its subharmonic. This mode has a wavelength twice that of the most unstable mode and is able to radiate.

5. The most unstable mode and its subharmonic are able to force other modes through triad-interaction to distribute the energy across different scales. This energy transfer process becomes more effective for a stronger nonlinearity.

6. Because of nonlinear energy transfer, a significant part of the total energy in EBC region injected by the external forcing is radiated to the west, although the unstable mode of the EBC cannot radiate. 


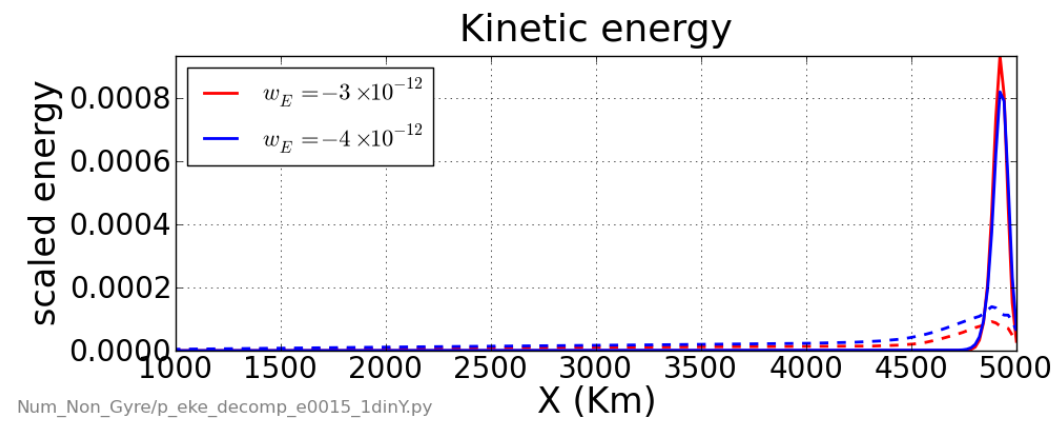

Figure 3-15: The zonal structure of the kinetic energy in $C 1$ (red lines) and $C 2$ (blue lines). The solid (dashed) lines show the kinetic energy of the mean and of eddies. The energy is scaled in such a way that $\iint E_{\text {jet }}(x, y) \mathrm{d} x \mathrm{~d} y=1$.

The analyses in this section support these conclusions but in an implicit way. There are uncertainties.

For example, we conduct two experiments to identify whether the longwave mode is an unstable mode for the EBC. In one experiment we filter out other modes but the longwave mode and the mean. It shows that the longwave mode diminishes and the mean current regains energy. During this process, the structure of the mean is altered because of the changes of the perturbation field. The new boundary current is not strictly the one in the experiment with all modes included. We then face a causality problem. Can the longwave mode be unstable in the all-mode-included case but stable in the filtered case? The short answer is NO. Detailed mechanisms are discussed in the next section.

\subsection{Mechanisms}

This section discusses the weakly nonlinear dynamics of an unstable EBC. In the weakly nonlinear regime, the system only supports a limited number of slowly growing modes. As a result, a discrete set of modes can be separated, and it becomes easier to identify their nonlinear interactions.

The model is able to simulate both the phase and amplitude of variables with very high accuracy. The linear and nonlinear behaviors of one or more specific modes can 
be studied by specifying compositions of the initial field, varying the model domain size, and turning on or off the nonlinear terms. More specifically, this section discusses the mechanisms that link the linear and nonlinear theories of an unstable EBC.

Instead of using an external forcing, we specify the EBC as a fixed background. The equation solved by the model in dimensional form is

$$
\nabla^{2} \psi_{t}+J\left(\psi, \nabla^{2} \bar{\psi}\right)+J\left(\bar{\psi}, \nabla^{2} \psi\right)+J\left(\psi, \nabla^{2} \psi\right)+\beta \psi_{x}=\nabla \cdot A_{h} \nabla \nabla^{2} \psi
$$

where the variables with overbars represent the fixed background.

For the truncated model, we write

$$
q=\sum_{n=-M}^{M} q_{n}(x, t) \exp (\text { in } \ell y), \psi=\sum_{n=-M}^{M} \psi_{n}(x, t) \exp (\text { in } \ell y)
$$

where $\psi_{n}=-\psi_{n}^{*}$ is the reality condition, and $M=(N-1) / 2$ for an odd $N$. If $N$ is even, then $n$ goes from $-N / 2$ to $N / 2-1$ including 0 . This is a typical discrete FFT decomposition. The inversion formula is

$$
D_{n} \psi_{n}=q_{n}, \quad D_{n}=\frac{\partial^{2}}{\partial x^{2}}-n^{2} \ell^{2}
$$

The $\ell$ is the wavenumber of the longest model-resolved wave defined as $\ell=2 \pi / W_{y}$, where $W_{y}$ is the model domain size in $y$. The linear Jacobians become

$$
J\left(\psi, \nabla^{2} \bar{\psi}\right)+J\left(\bar{\psi}, \nabla^{2} \psi\right)=i n \ell\left(\bar{v} D_{n}-\frac{\partial^{2} \bar{v}}{\partial x^{2}}\right) \psi_{n}
$$

and the nonlinear Jacobian becomes

$$
J(\psi, q)=J_{n} \exp (i n \ell y), J_{n}=i \ell\left[(n-m) \frac{\partial \psi_{m}}{\partial x} q_{n-m}-m \psi_{m} \frac{\partial q_{n-m}}{\partial x}\right]
$$


The equation for the $n^{t h}$ mode is

$$
\frac{\partial}{\partial t} q_{n}+J_{n}+\beta \frac{\partial}{\partial x} \psi_{n}=i n \ell\left(\frac{\partial^{2}}{\partial x^{2}} \bar{v}-\bar{v} D_{n}\right) \psi_{n}+\nabla \cdot A_{h} \nabla D_{n} \psi_{n}
$$

The first term on the rhs represents the energy transfer from the mean to perturbation field.

In the linear problem, the domain-integrated energy budget equation is

$$
\frac{\partial}{\partial t} E_{n}=\iint \psi_{n}^{*}\left[i n \ell\left(\frac{\partial^{2}}{\partial x^{2}} \bar{v}-\bar{v} D_{n}\right) \psi_{n}+A_{h} \nabla \cdot \nabla D_{n} \psi_{n}\right] \mathrm{d} x \mathrm{~d} y
$$

with $E_{n}=\frac{1}{2} \psi_{n}^{*} \psi_{n}$, which is merely another form of the energy budget equation 3.10. The rhs includes the energy transfer by the perturbation Reynolds stress and lateral friction. For an unstable mode $\psi_{n}$, the rhs yields a positive number which is the growth rate for that unstable mode. Given a fixed unstable boundary current, the linearly unstable modes will grow exponentially with energy input from the fixed basic state. We calculate the growth rates for a given mode $n$ by fitting its energy evolution to an exponential curve.

In the nonlinear problem, we consider truncating the model to $n=0, \pm 1, \pm 2$ for example. The nonlinear Jacobian terms become

$$
\begin{aligned}
J_{0} & =i \ell \frac{\partial}{\partial x}\left[\psi_{1}^{*} q_{1}-\psi_{1} q_{1}^{*}\right]+i 2 \ell \frac{\partial}{\partial x}\left[\psi_{2}^{*} q_{2}-\psi_{2} q_{2}^{*}\right] \\
J_{1} & =i \ell\left[\frac{\partial \psi_{0}}{\partial x} q_{1}-\psi_{1} \frac{\partial q_{0}}{\partial x}\right]+i \ell\left[2 \frac{\partial \psi_{1}^{*}}{\partial x} q_{2}+\psi_{1}^{*} \frac{\partial q_{2}}{\partial x}-\frac{\partial \psi_{2}}{\partial x} q_{1}^{*}-2 \psi_{2} \frac{\partial q_{1}^{*}}{\partial x}\right] \\
J_{2} & =i 2 \ell\left[\frac{\partial \psi_{0}}{\partial x} q_{2}-\psi_{2} \frac{\partial q_{0}}{\partial x}\right]+i \ell\left[\frac{\partial \psi_{1}}{\partial x} q_{1}-\psi_{1} \frac{\partial q_{1}}{\partial x}\right]
\end{aligned}
$$

with $q_{-n}=q_{n}^{*}$ to keep the fields real. Two terms in $J_{0}$ represent the feedback of the two modes to the mean field. $J_{0}$ equilibrates with the first term in $J_{1}$ and $J_{2}$. The direct coupling of mode $n=1$ and mode $n=2$ comes from the second bracket in $J_{1}$ and $J_{2}$. They act as new forcings to the original linear modes. Assume mode $n=1,2$ have intrinsic frequencies $\omega_{1}, \omega_{2}$ respectively, the nonlinear terms $J_{1}$ and $J_{2}$ 
will have new frequencies $\omega_{2}-\omega_{1}$ and $2 \omega_{1}$. The triad-resonance may occur if the new frequencies match the frequencies of the original modes, i.e., $\omega_{2}=2 \omega_{1}$. The resulting wave interactions can cause a rapid energy transfer/redistribution among different modes.

The triad-resonance in weakly nonlinear problems is a well-studied topic. Rigorous and systematic formulations can be found in previous studies, such as Phillips (1960) and Bretherton (1964). We here consider a special case of the nonlinear triadinteraction, in which two of the triad modes share the same frequency and wavenumber. This special case is the so-called subharmonic instability, which is explicitly identified in the following numerical simulations, and can be used to explain the results in the previous section.

\subsubsection{Linear growth rates}

The linear growth rates are first calculated as a reference for choosing parameters that are later used in the more controlled experiments. The background boundary current has a velocity profile $\bar{v}=-\bar{V} \operatorname{sech}^{2}\left(\frac{x+2 L}{L}\right)$, where $\bar{V}$ is a constant that sets the strength of the basic flow. The linearized version of equation 3.15 for the specified boundary current is solved by the same model used in Chapter 2 except that the friction term is also included here.

The model is run with the following parameters: $W_{x}=W_{y}=5000 \mathrm{~km}, \beta=$ $1.8 \times 10^{-11} / \mathrm{ms}$, the number of grid points in $x$ and $y$ are the same $N_{x}=N_{y}=256$, $A_{h}$ is the same as the one used in case $C 1, \bar{V}$ is $0.11 \mathrm{~m} / \mathrm{s}$.

The growth rate for each normal mode in linear stability theory can be reproduced by integrating the model with the corresponding normal mode initialization, then fitting the time evolution of the integrated perturbation energy to an exponential curve.

First we calculate the linear growth rates for the discrete wavenumber $\ell_{n}=n / W_{y}$ with $n=[8 \cdots 20]$. The corresponding wavelengths $\lambda_{n}=W_{y} / n$ range from $625 k m$ to $250 \mathrm{~km}$ given $W_{y}=5000 \mathrm{~km}$. 
Figure 3-16 shows the results. There are apparent longwave and shortwave cutoffs at wavelength $550 \mathrm{~km}$ and $250 \mathrm{~km}$ respectively. The most unstable mode has a wavelength about $340 \mathrm{~km}$.

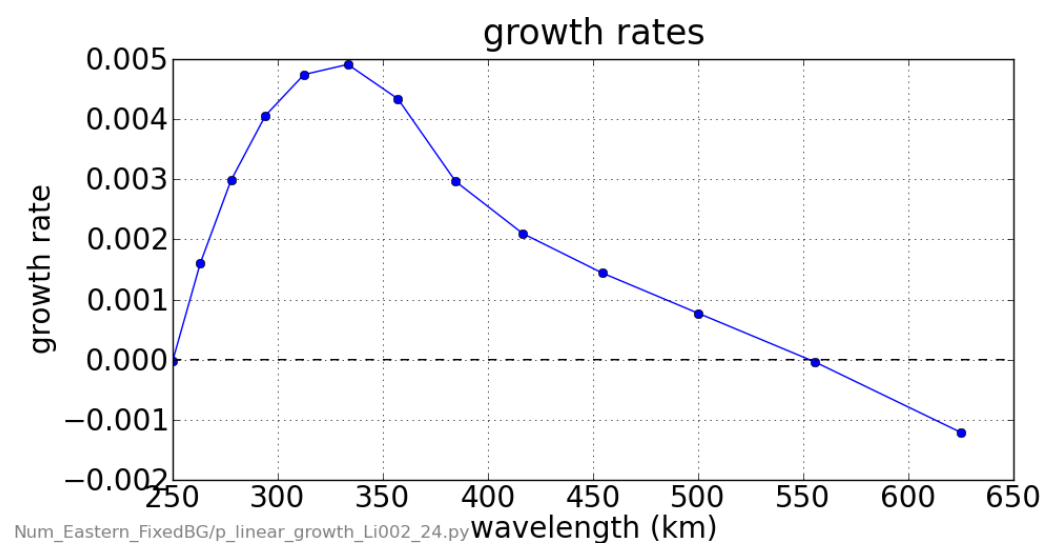

Figure 3-16: Linear growth rates of a boundary current as a function of meridional wavelength. The linear stability equation is solved by a numerical model. The growth rates are calculated by fitting the time series of the integrated perturbation kinetic energy to exponential curves, $E K E=C e^{\omega_{i} t}$, where $\omega_{i}$ is the growth rate. The growth rates shown in this figure are with units of /day.

As the model resolves modes with wavelength $W_{y} / n$, we can pick a limited number of unstable modes by varying $W_{y}$. For example, with $W_{y}=700 \mathrm{~km}$, the longest mode resolved in the model has a wavelength $\lambda_{1}=700 \mathrm{~km}$. For the second and third modes, $\lambda_{2}=350 \mathrm{~km}$ and $\lambda_{3}=233.3 \mathrm{~km}$. Examining the growth rate curve, one can find that only the second mode $n=2$ has a positive growth rate. This mode-picking is possible because unstable modes are confined in a narrow range in wavelength (between $550 \mathrm{~km}$ and $250 \mathrm{~km}$ ) for the given velocity profile and parameters. In the following, we set the model to support only one unstable mode, and test the two scenarios proposed in the last section.

\subsubsection{Case $\omega_{2}=2 \omega_{1}$}

In this experiment, we use the same set of parameters as those used in section 3.4.1, but with $W_{y}=700 \mathrm{~km}$ and $N_{y}=32$. Figure 3-17 shows the curve of the linear 
growth rates which is the same as in Figure 3-16 but for $W_{y}=700 \mathrm{~km}$. Because of the small $W_{y}$, only one unstable mode is represented by the model, which is $\psi_{2}$ with $\lambda_{2}=350 \mathrm{~km}$.

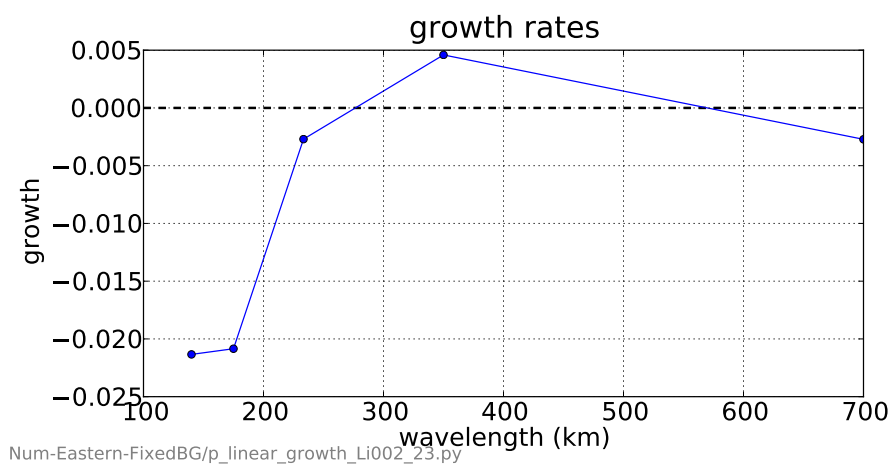

Figure 3-17: The same growth curve as shown in Figure 3-16, but for a case with $W_{y}=700 \mathrm{~km}$. Mode $\psi_{2}$ with $\lambda_{2}=350 \mathrm{~km}$ is the only unstable mode represented by the model. The first five modes are shown here.

Figure 3-18 shows streamfunction patterns for the mode $\psi_{1}$ and $\psi_{2}$ with wavelength $700 \mathrm{~km}$ and $250 \mathrm{~km}$. These are calculated by integrating a linear model with corresponding normal mode initializations. Notice that although $\psi_{1}$ is a decaying mode, it has an eigen-structure. Two modes have the same initial amplitude, $3.18 \mathrm{~m}^{2} / \mathrm{s}$ in terms of streamfunction, but $\psi_{1}$ becomes significantly weaker because of the negative growth rate. It is a radiating mode with a barely-westward-decaying streamfunction. The shorter wave mode $\psi_{2}$, however, is trapped around the boundary current region and has a positive growth rate as shown in Figure 3-17. We can expect that $\psi_{2}$ will excite resonance with $\psi_{1}$ if the frequencies of the two satisfy the requirement for subharmonic instability.

Examining the frequencies for different modes as shown in Table 3.1, we confirm that $\omega_{2}=2 \omega_{1}$, i.e., the frequency relationship between $\psi_{1}$ and $\psi_{2}$ satisfies the requirement for resonance. We also notice that the frequencies of the mode $n=2,3,4$ are negative, corresponding to negative phase speeds. The longer mode $\psi_{1}$, however, has a positive frequency (equivalently a positive phase speed), indicating that it is a 


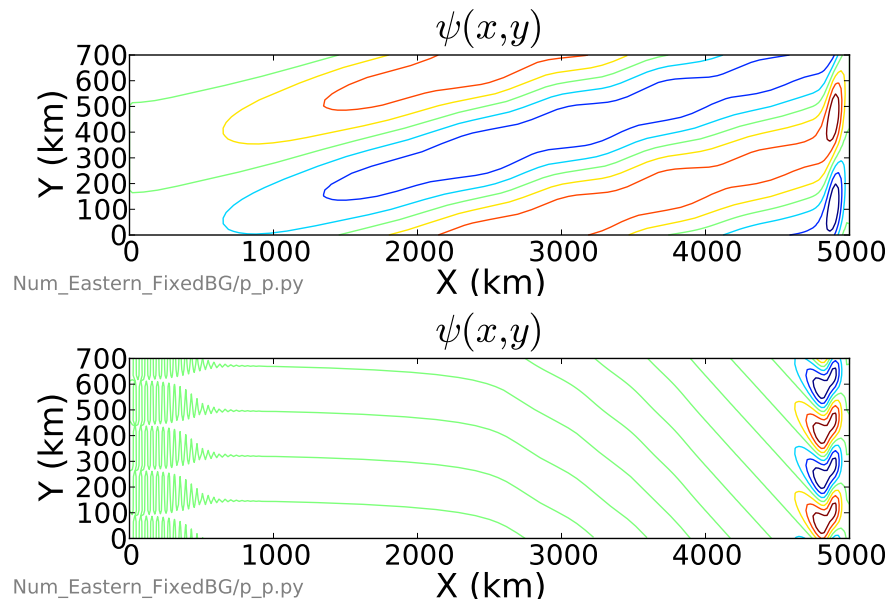

Figure 3-18: Stream-functions corresponding to $\psi_{1}$ with $\lambda_{1}=700 \mathrm{~km}$ and $\psi_{2}$ with $\lambda_{2}=350 \mathrm{~km}$ in Figure 3-17. They are normalized by the maximum value because they are linear modes.

\begin{tabular}{|c|c|c|}
\hline mode $\mathrm{n}$ & $\lambda(\mathrm{km})$ & $\omega$ (cycle/year) \\
\hline \hline 1 & 700 & 1.44 \\
\hline 2 & 350 & -2.88 \\
\hline 3 & 233 & -6.48 \\
\hline 4 & 175 & -10.08 \\
\hline
\end{tabular}

Table 3.1: Frequencies of the first four modes. Their growth rates are shown in Figure 3-17.

retrograde mode.

The criteria for the subharmonic instability are satisfied in the linear results. We now run the nonlinear model, where different modes can interact and we expect the resonance between $\psi_{1}$ and $\psi_{2}$.

The model performs the Fourier transform on $q$ at every time step during the integration. We save the Fourier coefficients for different modes, and plot the enstrophy $|\hat{q}(n, t)|^{2}$, as the index of the mode activity.

Figure 3-19 shows the results. The vertical axis is $\log _{10}$ scaled, so that linearly sloping lines indicate exponentially growing or decaying amplitudes and level lines indicate constant amplitudes. As expected from the linear study, the mode $n=2$ is the most unstable mode as shown by the green line. It grows fastest and reaches 


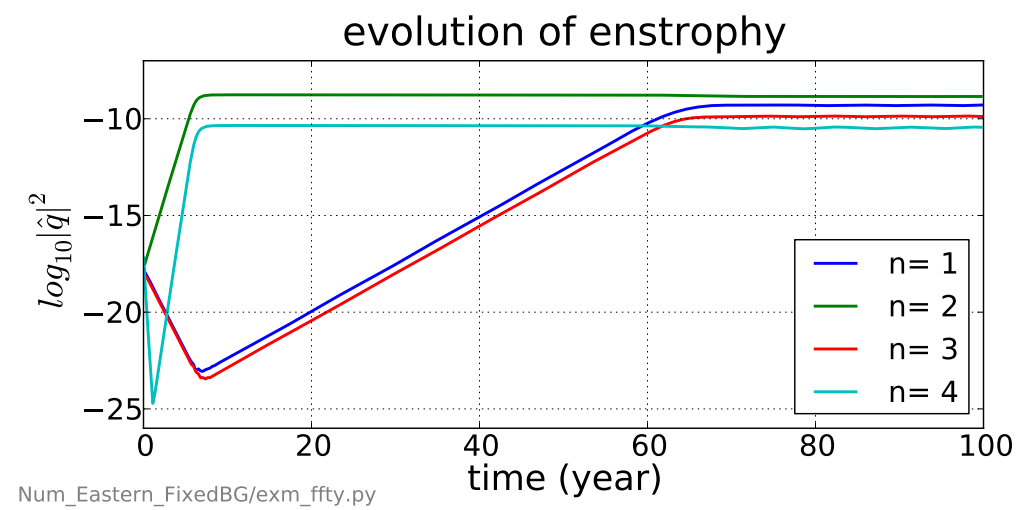

Figure 3-19: The time evolution of the enstrophy in the first four modes. The mode index $n$ is shown in the legend.

nonlinear equilibrium at about year 7 . The mode $n=4$ (cyan) is the superharmonic of mode $n=2$ in terms of wavenumber, and can be generated by the self-interaction of mode $n=2$. However, their frequencies do not satisfy the resonance relation, so that mode $n=4$ forced mode by mode $n=2$ without resonance. The initial adjustment of the model to the random noise initialization is indicated by the initial decay of mode $n=4$.

The aforementioned two modes have similar time evolutions of enstrophy. However, modes $n=1,3$ show different behaviors. The blue line represents the longest mode $n=1$. It first exponentially decays, then starts to grow exponentially as soon as mode $n=2$ reaches equilibrium. As its initial amplitude is small, it takes about 60 years for it to acquire an amplitude comparable to that of mode $n=2$. The system reaches equilibrium at about year 70 when the growth of mode $n=1$ is halted by nonlinear effects, which include the self-interaction and the feedback to the mean field. The finite energy in mode $n=3$ could be supported by mode $n=1$ and mode $n=2$ through the triad-interaction. But again, the triad $n=1,2,3$ in terms of wavenumber do not satisfy the frequency requirement for nonlinear resonance, so that mode $n=3$ is only a forced mode.

One should notice that these stages are separable because of the weakness of the forcing, the coarse spectral resolution, and the slow development of the instability 
(50 years for the second stage). The forcing scale and the model spectral resolution are reduced to such a level that the model supports only one unstable mode. The slow development of the instability shown in this section is an advantage rather than a defect, allowing the clear illustration of the physical processes involved. The development timescale will be significantly shortened once the forcing scale or the spectral resolution is increased for the system to support larger growth rates or more than one unstable mode. In a test experiment with the same spectral resolution, a $9 \%$ increase in the forcing scale shortens the timescale of the second stage to 7 years from the original 50 years. One may test the relationship between the forcing strength and the development timescale, but this relationship is not relevant to our purpose of illustrating the basic physical mechanisms. Strong nonlinearity can be achieved by using velocity profiles that support more unstable modes, or by including other energy sources, such as the available potential energy in a baroclinic current.

Figure 3-20 shows an example of the time series of the streamfunction at a fixed location and two-dimensional streamfunction snapshots at different stages. The time series at a fixed location shows that the periodic oscillation becomes stronger after the subharmonic mode $n=1$ gains a finite amplitude. The streamfunction before the nonlinear resonance become significant is shown in the middle panel, which mainly consists of the unstable trapped mode $n=2$. At later stage with a prominent nonlinear effect, the structure of the longwave mode $n=1$ appears and dominates in the interior.

The perturbation kinetic energy from the perturbation velocity field is shown in Figure 3-21. The green line shows the domain-integrated energy. The blue line represents the eastern-half-domain-integrated energy. The energy grows quickly following the growth of the unstable mode during the early developing stage, and reaches equilibrium at about year 7 . The unstable mode $n=2$ dominates for about 50 years, during which the perturbation energy is trapped around the boundary indicated by the overlap of the two lines. After the nonlinear resonance becomes important, the 


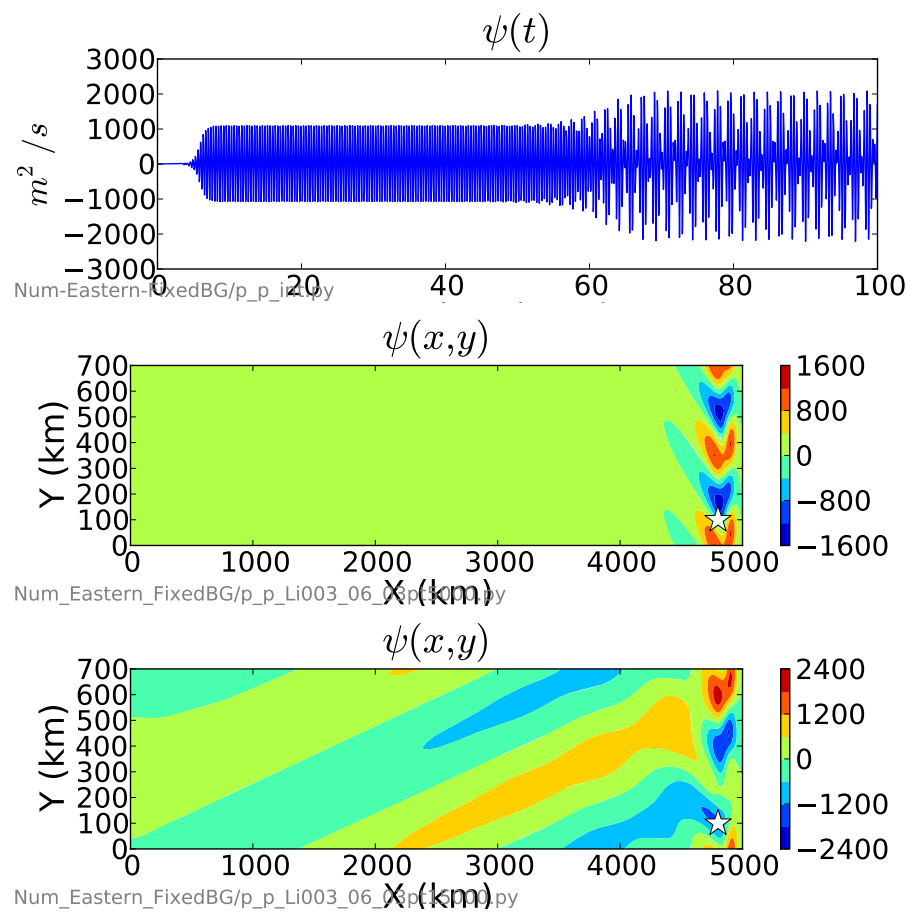

Figure 3-20: The upper panel shows the time series of the streamfunction at a fixed station indicated by the white star in the lower panel. The streamfunction snapshots at $t=40$ and $t=90$ are shown in the middle and lower panels.

perturbation energy reaches a new higher level. The increased perturbation energy is also indicated in the time series of the streamfunction at a fixed location shown in Figure 3-21. The large gap between the energy over the whole domain and the energy over the eastern half domain indicates that the radiating mode $n=1$ sheds energy away from the boundary region westward into the interior. This radiating mode is not an unstable mode of the specified boundary current, and does not exist without nonlinear interactions.

The energy budgets calculated according to equation 3.10 for a period of 20 years between year 20 and 40 (referred to as stage 1) and for a period of 20 years between year 80 and 100 (refereed to as stage 2) are shown in Figure 3-22. At stage 1, the main balance is between the energy input by Reynolds stress and the frictional dissipation. At stage 2, the divergence (mainly due to the beta flux) terms become important. Part of the energy is dissipated locally, and the remaining part is radiated away by 


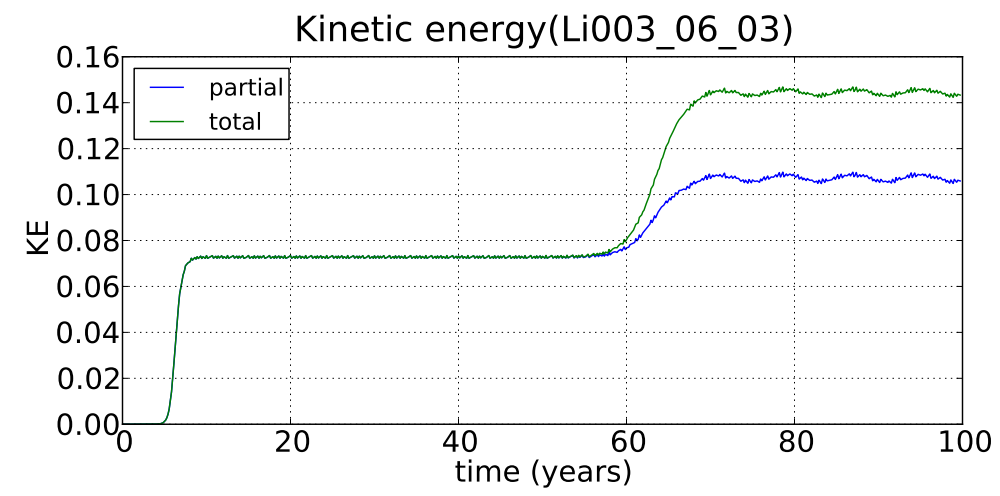

Figure 3-21: The perturbation kinetic energy integrated over the whole domain (green line) and the eastern half domain (blue line). The discrepancy between these two lines implies the influence of the radiating mode.

the beta flux and dissipated at the WBC.

The energy budget is closed for stage 1, which can be inferred from the zero total energy (the black line in the left panel), but has a small negative residual for stage 2 (the black line in the right panel), which may come from the higher derivatives of the mean field. The energy budget does not vary much in the streamwise direction because the boundary jet is parallel to $y$.

Next we examine the energy budget by carrying out a mode-decomposition and considering the energy budget for each mode. The energy budget equation for each mode is slightly different from the linear version represented by equation 3.10. There will be a term representing the nonlinear energy transfer between modes, which is in the form of $J_{n}$ in equation 3.19. The new energy budget with the nonlinear transfer considered is

$$
\begin{aligned}
\overline{\partial_{t} E_{n}^{\prime}} & +\nabla \cdot\left[\overline{-\psi_{n}^{\prime} \frac{D}{D t} \nabla \psi_{n}^{\prime}}+\overline{\overline{\mathbf{u}} E_{n}^{\prime}}+\overline{\mathbf{u}_{n}^{\prime} E_{n}^{\prime}}-\hat{x} \beta \overline{\frac{\psi_{n}^{2}}{2}}+A_{h} \overline{\psi_{n}^{\prime} \nabla \nabla^{2} \psi_{n}^{\prime}}\right] \\
+ & \nabla \cdot\left[\overline{\psi_{n}^{\prime}\left(\mathbf{u}_{n}^{\prime} \cdot \nabla\right)} \nabla \bar{\psi}+\overline{\psi_{n}^{\prime}(\overline{\mathbf{u}} \cdot \nabla) \nabla \psi_{n}^{\prime}}\right] \\
& =-\left(\overline{\left.\mathbf{u}_{n}^{\prime} \mathbf{u}_{n}^{\prime} \cdot \nabla\right)} \cdot \overline{\mathbf{u}}+A_{h} \overline{\nabla \psi_{n}^{\prime} \cdot \nabla \nabla^{2} \psi_{n}^{\prime}}-\overline{\psi_{n}^{\prime} J_{n}},\right.
\end{aligned}
$$

where $J_{n}$, the same term as in equation 3.19, represents the nonlinear energy transfer 

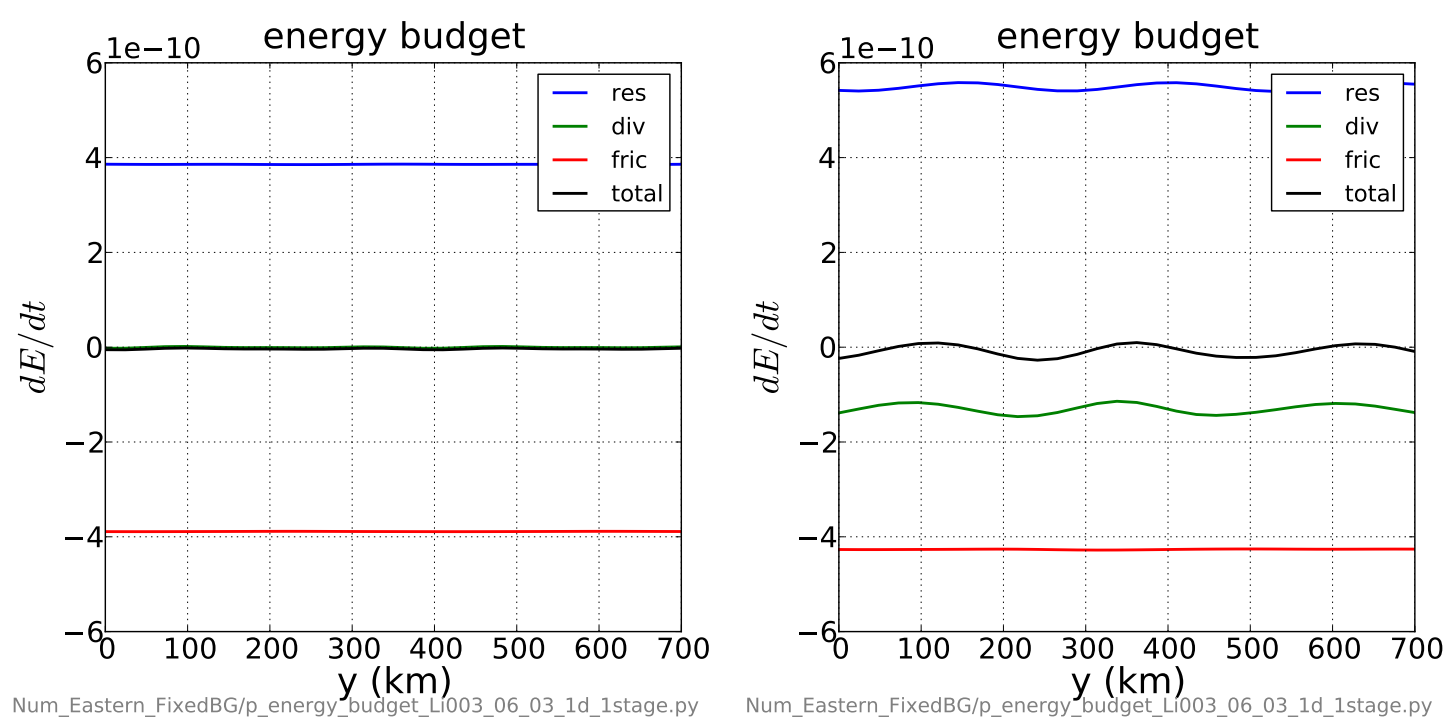

Figure 3-22: The streamwise structure of the energy budgets integrated over a zonal span of $500 \mathrm{~km}$ off the boundary during stage 1 (left) and stage 2 (right). A boundary region with $500 \mathrm{~km}$ zonal span is sufficiently large to enclose all energy input by Reynolds stress. The labels 'res', 'div', 'fric', and 'total' refer to the budgets by the Reynolds stress, divergence terms, frictional terms, and the total. The total is approximately zero, which means the budget is closed. The budgets have no significant $y$-dependent structures because the boundary jet is parallel to $y$. The balance is mainly between the input by the Reynolds stress and the frictional dissipation during stage 1. But the divergence terms (mainly due to the beta flux) become important during stage 2. Part of the energy is dissipated locally, and the remaining part is radiated away by the beta flux.

by all possible triad-interactions. Here we are interested in the interaction between mode $n=1$ and 2 , because we already know that the major nonlinear interaction happens between these two modes.

The $y$-averaged energy budgets for the total perturbation field and mode $n=$ 1,2 at different stages are listed in Table 3.2. The labels, 'res', 'fric', 'div', and 'non' represent the terms due to Reynolds stress, friction, advection and waves, and nonlinear transfers respectively. We rescale all energy terms with respect to the total energy input by Reynolds stress at each stage. As a result, we see that the contributions of Reynolds stress, denoted as 'res', are 1 at both stages.

At stage 1 , as we already know, there is only one dominant mode, the mode $n=2$. 


\begin{tabular}{|c||c|c|c|c|c|c|}
\hline \multicolumn{1}{|c||}{ Budget } & \multicolumn{3}{c|}{ Stage 1 } & \multicolumn{3}{c|}{ Stage 2 } \\
\hline \hline terms & total & $\mathrm{n}=1$ & $\mathrm{n}=2$ & total & $\mathrm{n}=1$ & $\mathrm{n}=2$ \\
\hline res & 1 & 0.0002 & 0.997 & 1 & 0.3 & 0.7 \\
\hline fric & -1.0083 & -0.0001 & -0.98 & -0.7750 & -0.14 & -0.57 \\
\hline div & -0.0016 & -0.0001 & -0.004 & -0.2597 & -0.25 & -0.007 \\
\hline non & -- & $+10^{-6}$ & $-10^{-6}$ & -- & 0.1307 & -0.1316 \\
\hline
\end{tabular}

Table 3.2: The energy budgets at different stages averaged over a $500 \mathrm{~km}$-wide boundary box. The total Reynolds stress energy input is $3.85 \times 10^{-10}$ at the first stage and $5.48 \times 10^{-10}$ at the second stage. All values in the table are ratios to the total energy input by Reynolds stress at their own stage. The longwave mode is weak at the first stage, but becomes significant at the second stage. It consequently alters the route of energy transfer.

This mode is responsible for $99.7 \%$ of the total Reynolds stress energy input, and for about the same amount of energy that is dissipated. Mode $n=1$ has negligible energy contribution either in energy input or loss. The nonlinear energy transfer between these two modes is infinitesimal. The budget is not closed in an exact sense because of the low sampling frequency and numerical errors in the diagnostic calculations, especially when higher order derivatives are involved.

At stage 2 , both mode $n=1$ and $n=2$ become important in the energy balance. About 30 percent of the total energy input by Reynolds stress is done by mode $n=1$, and 70 percent is done by mode $n=2$. Overall, friction accounts for 77.5 percent of the energy removal for the boundary region. Most of this 77.5 percent is due to mode $n=2$. The remaining energy removal (out of the boundary region) is done by radiation (mainly due to the beta flux). Most of this energy radiation is done by mode $n=1$. The energy budgets for each mode are far from being closed. That is because the nonlinear energy transfer between the two modes becomes significant. For mode $n=1$, more energy is needed to support its equilibrium. However, the nonlinear energy transfer has no net contribution to the total energy budget because each energy gain in mode $n=1$ corresponds to an equal energy loss in mode $n=2$. We notice that numerical errors do exist in these calculations as the total energy loss $($ fric + div $=-1.035)$ is $3.5 \%$ more than the total energy gain $($ res $=1)$. This energy 
budget is calculated over a boundary region. Obviously, the energy radiation only redistributes energy, but does not contribute to the ultimate energy removal. The friction will be the only energy sink in a domain-wide energy budget.

Mode $n=1$ has a positive contribution to the energy input. It means that given a finite amplitude, mode $n=1$ can draw energy from the mean current although it has a negative growth rate in the linear calculation. It is not self-sustained because the energy loss by dissipation and radiation is larger than the energy input from the mean current. However, it is puzzling that the linearly stable long wave is able to extract so substantial an amount of energy from the mean. One possibility is that the short wave alters the long wave's structure in $x$ so that the long wave acquire a Reynolds stress that can extract more energy from the mean. This hypothesis is tested by an extended experiment based on that in this subsection. The model is truncated to have only modes $n=0,1$, i.e., only the mean and the longwave mode, initialized with the final state of the full model at year 100, and run for another 6 years. The structure change can be detected by comparing the state at year 100 and year 106 .

Figure 3-23 shows the results. The upper panel shows the streamfunction of the longwave mode in the initial state (the nonlinear equilibrium state at year 100). The middle panel shows the streamfunction of the longwave mode at the end of year 106. This structure is different from the one in the nonlinear equilibrium state shown in the upper panel. The nonlinear energy transfer from the shortwave mode to the longwave mode is cutoff by the truncation. The elimination of the short unstable mode results in a decaying longwave mode and also a decaying total perturbation kinetic energy (the lower panel). Note that the changes of the longwave mode appear not only over the boundary region but also over the interior. This indicates that the shortwave mode alters the longwave mode within the boundary region in terms of both the structure and the dispersion relation.

This experiment shows that, in a strict sense, the modes in the nonlinear simula- 

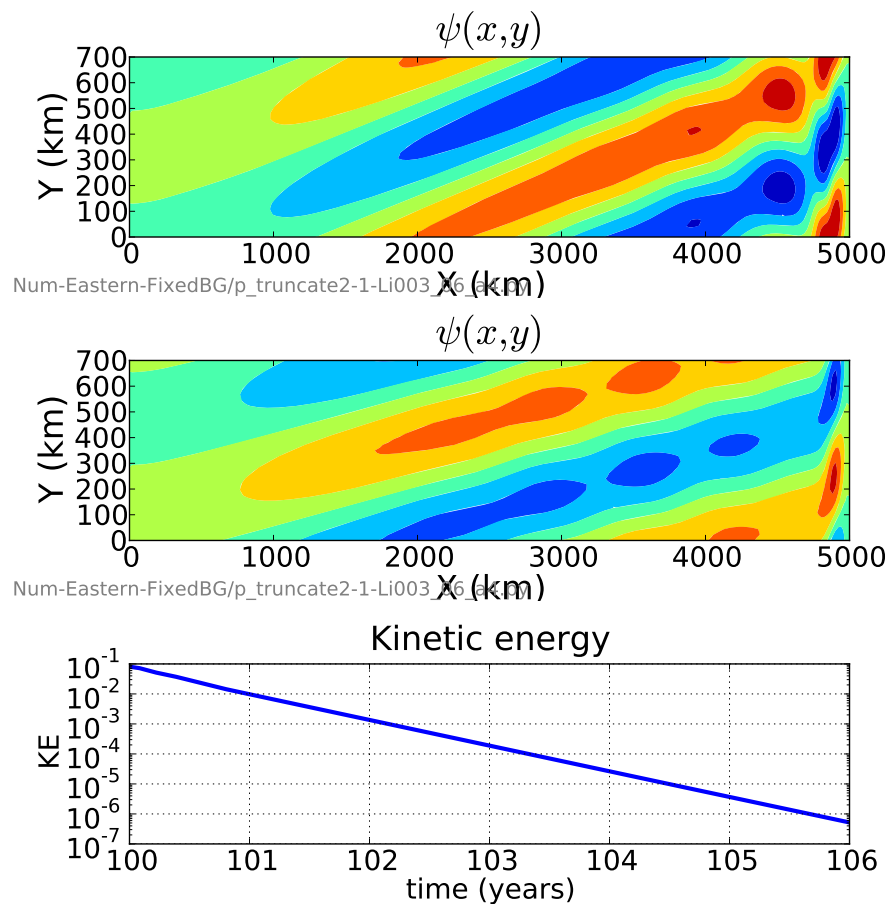

Figure 3-23: The streamfunction structure of the longwave mode at year 100 (upper) and year 106 (middle). They are reconstructed from the total field using FFT, and are normalized by their maximum values. The lower panel shows the time series of the total perturbation kinetic energy. The model is truncated to only have the mean and the longwave mode. The nonlinear energy transfer from the shortwave mode to the longwave mode is cutoff by the truncation. The kinetic energy of the longwave mode decays in time.

tions are slightly different from the modes in the linear analysis. The unstable mode can alter the long, stable mode to have a Reynolds stress that can extract energy from the mean. The longwave mode at finite amplitude is not sustainable without the support of the shortwave mode through the nonlinear wave-wave interaction.

Another nonlinear effect is that the finite amplitude perturbations feed back to the mean to change the structure of the basic boundary current. The affected boundary current will certainly change the stability properties and yield different eigen-modes. The new eigenmodes do not satisfy the requirement for triad-resonance, so that the two harmonics decouple, leading to a weaker perturbation field. The boundary current will then relax to its unaffected state, generating again the more matched harmonics to start a new cycle. This mechanism can be detected from Figure 3-21, where the 
energy oscillates during the later stage after year 70, and it can also explain the "breathing" feature shown in Figure 3-11.

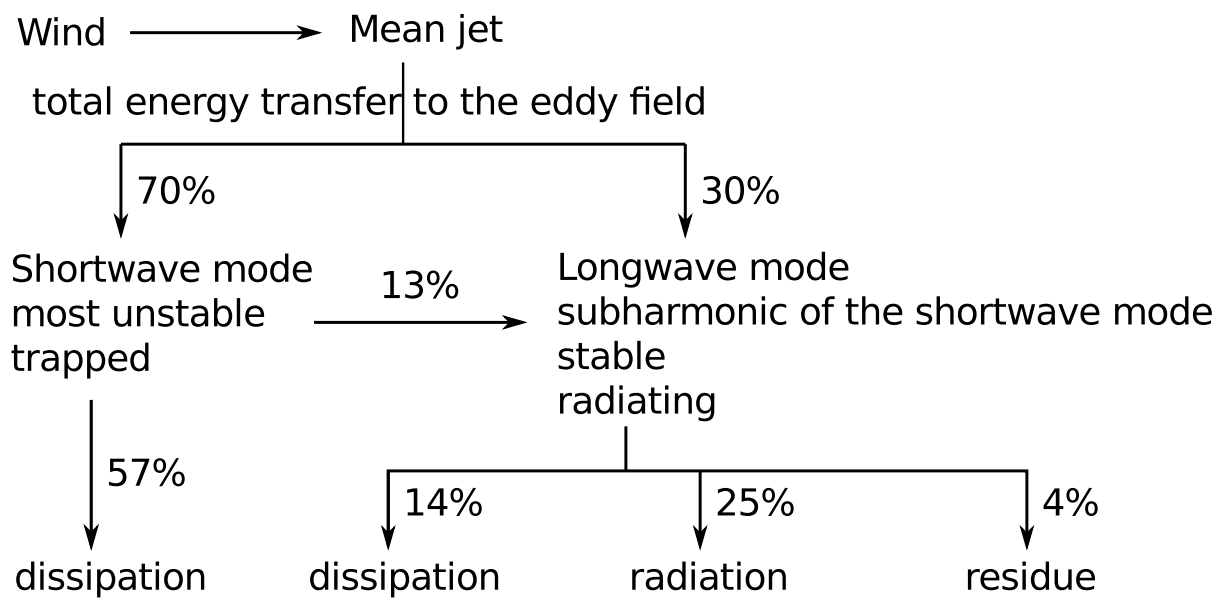

Figure 3-24: The schematic diagram showing the route of energy transfer at stage 2.

We summarize the route of the energy transfer at stage 2 by the diagram shown in Figure 3-24. The mean boundary jet is supported by the external forcing. It becomes unstable and transfers energy into the perturbation field. 70 percent of this energy goes to the most unstable mode, and 30 percent goes to the long radiating mode. The longwave mode can directly draw energy because it is altered nonlinearly by the shortwave mode. Of the energy that goes into the trapped unstable mode, 57 percent is dissipated locally, and 13 percent goes into the longwave mode through nonlinear energy transfer. The long radiating mode, with the support of the nonlinear energy transfer from the trapped mode, dissipates 14 percent and radiates 25 percent of the total energy. Overall, 57 percent of the total energy loss is due to the trapped mode, and 43 percent is due to the radiating mode. The linearly decaying mode and the most unstable mode become equally important in the energy budgets.

\subsubsection{Case $\omega_{2} \neq 2 \omega_{1}$}

We keep the model setup in this experiment the same as that used in $\omega_{2}=2 \omega_{1}$ case, but only increase $\beta$ from $1.8 \times 10^{-11} / \mathrm{ms}$ to $2 \times 10^{-11} / \mathrm{ms}$. The change of beta alters 
the frequency of mode 1 , which will be shown later, so that the frequency requirement for nonlinear resonance, $\omega_{2}=2 \omega_{1}$, is not satisfied. Figure 3-25 shows the growth rates for the first five modes. Similar to that shown in Figure 3-17, only mode $n=2$ has a positive growth rate.

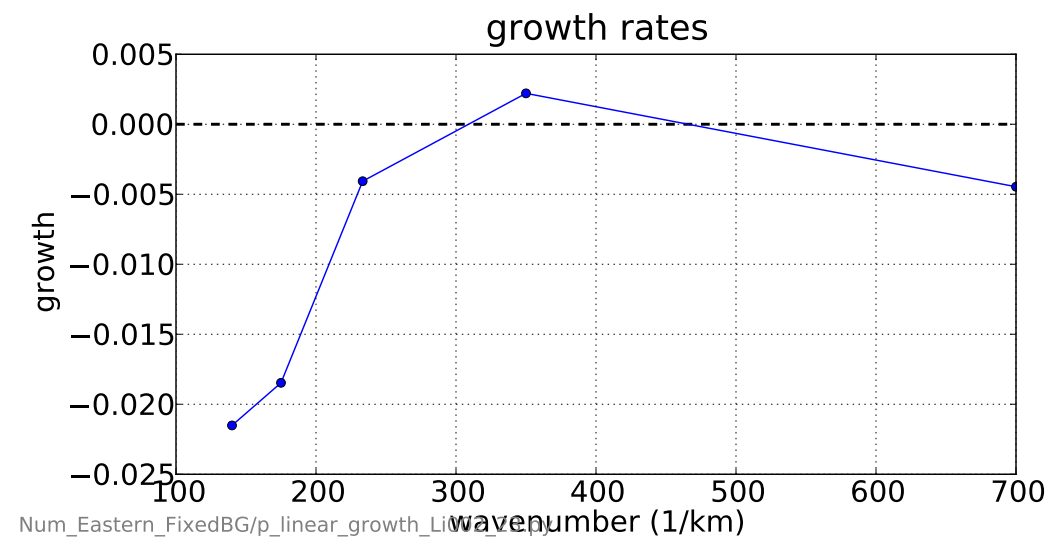

Figure 3-25: The same as Figure 3-17 but for $\beta=2 \times 10^{-11} / \mathrm{ms}$ case.

Figure 3-26 shows the time series of the perturbation streamfunction at a random location, a snapshot of the perturbation field in the equilibrium state, and the time evolutions of enstrophy for the first four modes. The evolution of enstrophy shows that the unstable mode, $n=2$, quickly stands out from the initial random noise, and dominates the system after that. Mode $n=1$ and 3 decay until their values reach the machine precision. Mode $n=4$ is sustained by the nonlinear self-interaction of mode $n=2$, but with a negligible amplitude. The two-dimensional snapshot and the one dimensional time series of the streamfunction shown in the upper and middle panel are dominated by mode $n=2$.

The frequencies for the first four modes are listed in Table 3.3, showing a substantial mismatch between $\omega_{2}$ and $2 \omega_{1}$. As a result, the resonance criterion is not satisfied, and no significant nonlinear interaction happens between these two modes.

The energy budget for this case is similar to that during the stage 1 in the previous case, so is not repeated here. 

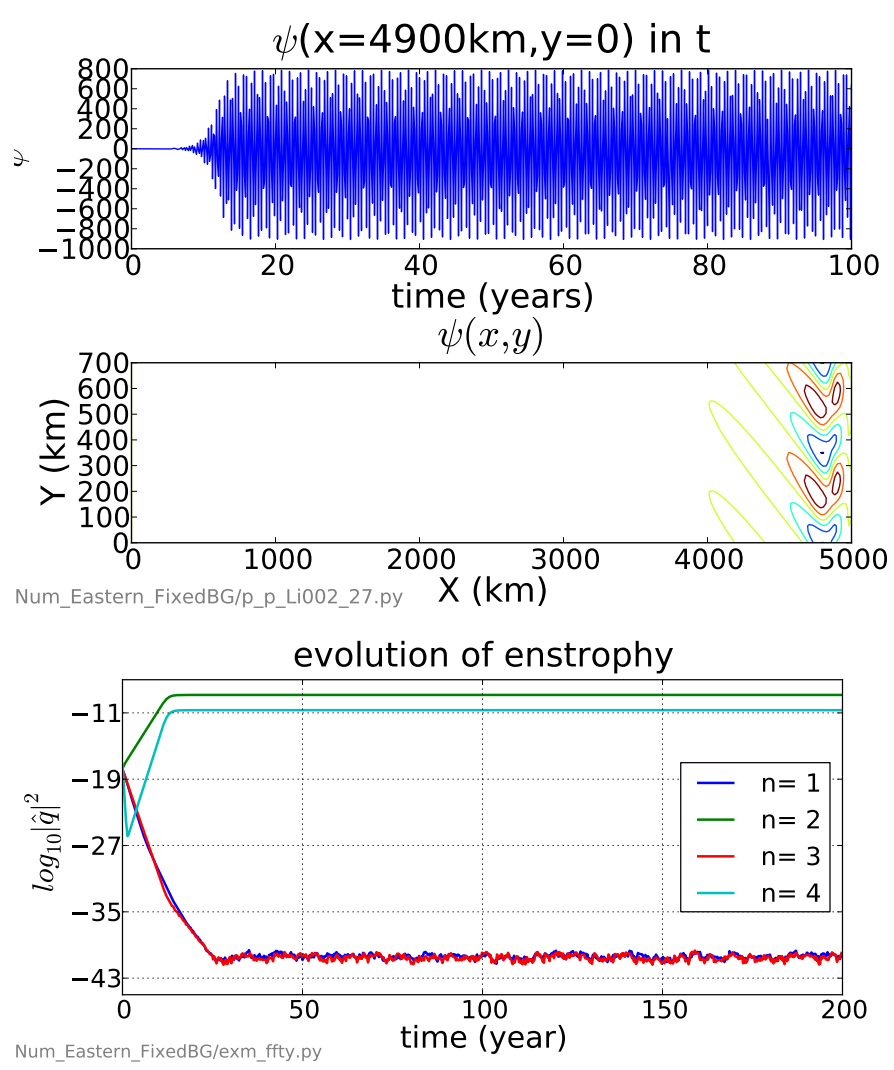

Figure 3-26: This figure shows the time series of the streamfunction at a random location inside the boundary current in the upper panel, a snapshot of the perturbation streamfunction at $\mathrm{t}=70$ years in the middle panel, and the time evolution of enstrophy in the first four modes in the lower panel.

\begin{tabular}{c|c|c}
\hline mode $\mathrm{n}$ & $\lambda(\mathrm{km})$ & $\omega$ (cycle/year) \\
\hline 1 & 700 & 2.16 \\
\hline 2 & 350 & -2.88 \\
\hline 3 & 233 & -6.48 \\
\hline 4 & 175 & -10.08 \\
\hline
\end{tabular}

Table 3.3: Frequencies for the first four modes. No possible combination for triadresonance exists. 


\subsection{Conclusion and discussion}

In this chapter, a series of controlled numerical experiments are conducted to study the nonlinear instability of an EBC and the transition from linear to nonlinear instabilities. It is found that the linearly decaying and radiating mode is subject to subharmonic instability and can play an important role in the energy budget of an EBC.

The transition from linear to nonlinear instability can be described by the spin-up process. Once the wind forcing (Ekman pumping) is turned on, the ocean adjusts to the forcing field following the Sverdrup relationship. The boundary current forms quickly, in about thirty days. After it spins up, the linearly most unstable mode stands out quickly and grows exponentially. Unstable modes exist because the structure of the boundary current is chosen to be linearly unstable. If the boundary current is weakly nonlinear and supports only one unstable mode, the mode will be saturated by nonlinear effects once it gains a finite amplitude. Depending on model parameters, the unstable mode can excite a longer mode through subharmonic instability. The longer mode is more able to radiate, contributes to perturbation energy budgets through radiating energies out of the boundary region. In this way, the linearly stable longwave mode becomes important in a nonlinear context.

The subharmonic instability observed here is essentially the secondary instability induced by triad-resonance. The secondary instability has been widely studied, and become a prototype of transitional boundary-layer instability in fluid dynamics field. One can find an early study of this topic in Craik (1971) and Orszag and Patera (1983), and a wonderful review in Herbert (1988).

Secondary instability occurs after primary instability is saturated. The primary instability as defined in Herbert (1988) refers to the initial stage of linear instability. It can be formulated into an eigenvalue problem and predicted for temporarily growing disturbances as is done in the previous chapter. The saturation is reached when the exponential growth of the unstable mode is halted by nonlinear effects. In the 
boundary layer instability theory as discussed in Herbert (1988), the second instability usually refers to the instability of three-dimensional perturbations to a saturated two dimensional wave. A meridional boundary current system on a beta plane with a saturated unstable mode is unstable to small perturbations if the subharmonic of the unstable mode (in terms of both wavenumber and frequency) is an intrinsic mode of the system. The saturated system stays in equilibrium (as seen in section 3.4.3) unless the secondary (subharmonic) instability happens (as seen in section 3.4.2).

In this chapter, the subharmonic instability is identified as the main mechanism that governs the linear to nonlinear transitional instability of an eastern boundary current. However, subharmonic instability is only a special case of the nonlinear resonant instabilities. More general triad-interaction can become equally important when the nonlinearity increases.

The weak nonlinearity also results in the absence of separated eddies in the nonlinear simulations presented in this chapter. To a large extent, the perturbation fields in these nonlinear simulations comprise only a limited number of linear modes. These discrete linear modes are less able to form energetic isolated eddy structures. However, isolated eddies are expected to form when the nonlinearity of the system is increased. This increase can result from a stronger forcing, a more unstable boundary current velocity profile, a smaller planetary beta, or more energy sources, such as the available potential energy in a baroclinic current.

Isolated eddies can be found in previous studies, which use the baroclinic models. Bracco et al. (2008) studied the dynamics of the eastern boundary current of the Labrador Sea using a quasi-geostrophic three layer meridional channel model. The model is able to reproduce the dominant features of the observed vortex structures along the west coast of Greenland. The local baroclinic instability plays a major role in generating the coherent vortices. Spall (2010) studied the water mass transformation and circulation in an idealized Nordic Seas using a primitive equation model with a surface thermal forcing. The warm isolated eddies are generated by a strong baroclinic eastern boundary current, and shed away from boundary region to the in- 
terior. Furthermore, increasing the baroclinicity of the boundary current results in stronger eddies. Both studies demonstrate the importance of the baroclinic energy in generating separated eddies.

The problem becomes much more complex if the barotropization process of the baroclinic eddies is considered, as observed and simulated in Haney et al. (2001). Haney et al. (2001) argue that the rapid seaward decrease of the eddy kinetic energy in the California Current is due to the surface weakening effect of the downward energy redistribution brought by the barotropization process. However, can the radiating instabilities also play roles in weakening the seaward eddy kinetic energy? It may provide another mechanism explaining the observed characteristics of the offshore propagating eddies.

The bottom topography is also an important ingredient in EBC systems. Spall (2010) show that a steeper topography induces a stronger vertical shear in the boundary current, and consequently a stronger eddy field. Bracco et al. (2008) show that perturbations are generally trapped along the boundary by the sloping bottom, but the meridional variation of the topography could enhance the westward shift of the isolated vortices. The westward movement of the isolated eddies is different from the westward radiation of instabilities. It is unclear whether and how these two mechanisms couple with each other.

The effect of the irregular alongshore topography on the westward movement of the vortices shown in Bracco et al. (2008) can be applied to associate the radiating instabilities from the EBCs with the generation of the zonal jets in the ocean interior discovered by Maximenko et al. (2005). It is originally suggested in the linear study by Hristova et al. (2008) that the zonal jets may be incited by radiating instabilities from an EBC. It is further demonstrated in this chapter that nonlinear radiating instabilities have small zonal wavenumbers, and show zonal jet structures extending thousands of kilometers westward into the interior. The radiating instabilities are transient waves, while the observed zonal jets are semi-permanent. The model of a parallel EBC alone, as the one used in this study, can not explain the stationary 
zonal jets because the amplitude of a transient wave field reduces to zero after applying a longtime average. However, this situation may change after considering the irregularity of the coastal bottom topography or coastlines. It is well studied and accepted that the irregularity of the coastline and topography is very important for anchoring coastal filaments and for enhancing the growth of meanders and eddies (Kelly, 1985; Batteen, 1997; Brink and Cowles, 1991). Although it is unclear how irregular coastlines and bottom topography affect the radiating instabilities, one can expect that the longtime average of radiating instabilities in the interior will have a finite amplitude because of the spatially irregular but stationary continental coasts. The time-mean field can then show traces of the long radiating modes emitted from the EBCs. The effects of bottom topography and coastline shape on the radiating instabilities are important and interesting topics for future studies. The topographic effect can become much complex in a baroclinic environment as demonstrated in Spall (2010). 
THIS PAGE INTENTIONALLY LEFT BLANK 


\section{Chapter 4}

\section{Instability of an eastern boundary current influenced by a large-scale flow: a linear study}

\subsection{Introduction}

The previous chapter discussed the barotropic instability of meridional jets. The jet profile is assumed to be invariant in the streamwise direction. This approach leads to the mathematical simplicity because the meridional component of the solution can be represented by normal modes of trigonometric functions. However, a large-scale flow can modify the boundary currents and affect their instabilities. This chapter investigates these influences in a linear regime. Figure 4-1 shows the conceptual model with the two components: a large-scale flow and an eastern boundary current.

The traditional approach to the dynamical instability of geophysical fluid systems is to consider a parallel basic flow, and study the behavior of the superimposed normal modes represented by products of trigonometric functions in time and streamwise direction. Substituting the normal mode solution into the linearized equation yields an eigenvalue problem, where the cross-stream structure is represented by the 


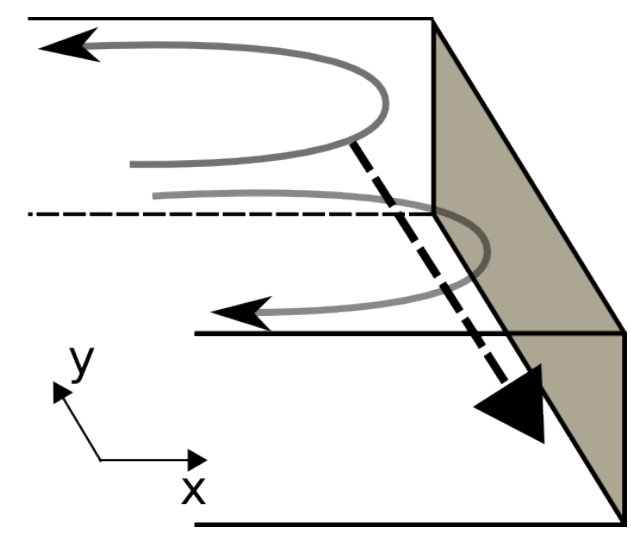

Figure 4-1: The conceptual model. There are two main elements: the background large-scale flow, the boundary current.

eigenfunction and the growth rate by the imaginary part of the complex frequency.

The large-scale flow, however, changes the picture of the conventional linear stability analysis of a steady parallel flow. The influence is twofold. Firstly, the large-scale flow field can continuously change the width of the basic boundary current, so that properties of the boundary current, such as the background shear, change with time and location. Secondly, the deformation field itself contributes to the streamwise variation of the boundary current. As a result, solutions of the system with a non-parallel boundary current are no longer trigonometric normal modes. Streamwise variations exist in the instability of the boundary jet. Furthermore, a meridional boundary current supports radiating instabilities through Rossby waves, which can be modified by the large-scale background flow. A non-uniform meridional boundary current and a large-scale interior circulation form a complex and interesting problem.

To study the linear influence of the large-scale flow on the boundary current, we assume the boundary current and the large-scale flow are maintained by an external forcing, so that they are fixed in time.

We first review basic concepts in Section 4.2, then formulate the problem in Section 4.3. The WKB solution and the time-dependent numerical solution of the linear problem are presented in Section 4.4 and Section 4.5. Section 4.6 gives the conclusion and discussion. 


\subsection{Background review}

In this section, we review several concepts upon which our discussions are built. Those concepts are grouped into three pairs and presented in the following three subsections.

\subsubsection{Temporal and spatial instability}

Temporal instability is traditionally used in the linear stability analysis of sheared flows. However, the spatially instability is more useful for spatially developing flows. We briefly review these two concepts in the following.

The linearized stability equation based on a parallel meridional flow has normal mode solutions in temporal and meridional coordinates. Together with proper boundary conditions, the stability equation yields an eigenvalue problem. Look for solutions in the form of

$$
\psi=\phi(x ; R) e^{i(\ell y-\omega t)}
$$

where $R$ are the control parameters, $\ell$ and $\omega$ are the wavenumber (meridional wavenumber for the case with a meridional jet considered in this thesis) and the frequency, and $\phi$ is the eigenfunction corresponding to the eigenvalue $\ell$ or $\omega$. The wavenumber and frequency are linked by a dispersion relation $\omega=\Omega(\ell)$ derived from the linear operator in the stability equation.

To discuss the linear stability, one has to choose between the temporal and spatial theory. In the temporal stability theory, it is assumed that perturbations in certain spatial patterns evolve in time. As a result, the wavenumber is assumed to be real and the goal of the linear theory is to determine the complex frequency as a function of the real wavenumber. This is the conventional approach for the stability analysis in geophysical fluid dynamics. In the spatial stability theory, however, the frequency is assumed to be real and the wavenumber is complex. It resembles spatially amplifying waves generated by a localized sinusoidal forcing at a given frequency.

One can certainly carry out the temporal stability analysis for the spatially varying

flow. However, we are more interested in how a wavemaker-generated signal, or a wave 
packet, evolves in a spatially varying flow. The spatial instability concept is more suitable for our interests.

The two theories are formalized as follows.

Temporal stability:

$$
\ell \in \mathbb{R} \text { and } \omega \in \mathbb{C}
$$

Spatial stability:

$$
\ell \in \mathbb{C} \text { and } \omega \in \mathbb{R}
$$

where $\mathbb{R}$ and $\mathbb{C}$ represent the set of real and complex numbers.

There is no direct linear transformation between the temporal and spatial growth rates. However, Gaster (1962) show that when the growth rates are small $c_{i} \rightarrow 0$, the link between these two at leading order is

$$
\frac{\omega_{i}}{\ell_{i}}=-\frac{\partial \Omega_{r}}{\partial \ell_{r}}=c_{g}
$$

where $\omega_{i}$ is the temporal growth rate, $\ell_{i}$ the spatial growth rate, $\Omega_{r}$ the characteristic function describing the dispersion relation, and $c_{g}$ the group velocity of the mode with a wavenumber $\ell_{r}$. We will revisit this relationship in section 4.5.1, where results of the eigenvalue problem and results of numerical simulations are compared. The spatial growth rate $\ell_{i}$ can be solved directly in the eigenvalue problem given a real frequency $\omega_{r}$. The process is similar to the one used in the temporal instability, where the complex frequency is solved given a real wavenumber.

In the temporal stability problem of an inviscid system, the complex eigenvalue, $c_{i}$, usually appears in conjugate pairs. We are more interested in the eigenvalues with positive imaginary parts as they represent the exponential growth. In the spatial stability problem, there are two branches in terms of wavenumber given a second order ordinary stability equation. One of the eigenvalues represents a spatially amplifying mode and the other represents a decaying mode. We use Brigg's rule which was first described in Briggs (1964), to identify the eigenvalue of the amplifying mode. 
Given a real frequency $\omega_{r}$ and a complex eigenvalue $\ell=\ell_{r}+\mathrm{i} \ell_{i}$, the solution is written as

$$
\psi=\phi(x ; R) e^{-\ell_{i} y} e^{i\left(\ell_{r} y-\omega_{r} t\right)}
$$

As described earlier, the spatially growing mode can be obtained by studying the signaling problem, where amplifying waves are generated by a wavemaker at a specific location, say $y=0$. For amplifying modes, it requires $\ell_{i}>0$ over the region $y<0$, and $\ell_{i}<0$ over the region $y>0$. We choose the $\ell_{i}$ for amplifying modes by determining the region where the signal propagates. If the group velocity is negative, the perturbation generated at $y=0$ will propagate into the region $y<0$ and vice versa. As a result, the $\ell_{i}$ that represents the spatially amplifying mode is determined by the sign of the group velocity associated with the forcing frequency $\omega_{r}$. We know from the previous chapter that $c_{g}$ of an unstable mode is negative in a barotropic southward Bickley jet along the eastern boundary, then the eigenvalue $\ell$ with a positive $\ell_{i}$ represents the spatially amplifying mode.

Briggs' criteria is based on the same reasoning but uses a different approach, which is necessary when the sign of the group velocity is not known a prior. Consider a spatially unstable system driven by a localized wave maker with frequency $\omega_{r}$. Let the perturbations propagate and grow to form a spatially amplifying structure. If the system is driven by a source that is localized in space and has an exponentially growing sinusoidal time dependence, the wave should exhibit a structure that decays away from the source if the source grows in time with a sufficiently large growth rate. Mathematically, whether or not a given mode with a complex wavenumber $\ell=\ell_{r}+\mathrm{i} \ell_{i}$ for a frequency $\omega_{r}+\mathrm{i} \omega_{i}$ is amplifying is determined by whether or not $\ell_{i}$ changes sign following the increase of the positive imaginary part of the forcing frequency. If $\ell_{i}$ does change sign, then the wave is amplifying, otherwise, the wave is evanescent.

In this chapter, we use the concept of the spatial instability to address the influence of the large-scale circulation on the stability of a boundary current. 


\subsubsection{Local and global mode}

As is defined in Huerre (1990), the terms local and global refer to the instability of the local field and of the entire flow field, respectively. Obviously local and global modes are important for spatially-varying flows, and there is no need to distinguish the local and the global mode for a parallel flow.

In the discussion of the local mode, the concepts of absolute and convective instability are introduced based on the impulse response of the system at each streamwise location. The generation of the global mode for a spatially varying flow is also linked to the absolute and convective instability.

\subsubsection{Absolute and convective instability}

First of all, we should point out, as mentioned by Pierrehumbert (1984), that the term convective refers to the wave packet propagation rather than any kind of thermally driven instability. The term was initially introduced in the area of plasma physics, where there is no confusion of phenomena with thermally driven convection.

The concepts of the absolute and convective instability are based on the local mode behavior. If a localized disturbance or an impulse perturbation grows and spreads upstream and downstream, then the flow is said to be locally absolutely unstable. In other words, a fixed station is called absolutely unstable if the response of the local system to a small disturbance grows as $t \rightarrow \infty$. If the growing disturbances are swept away from the source, the flow is said to be locally convectively unstable (Huerre and Monkewitz, 1990).

Following earlier analysis (Briggs, 1964; Bers, 1975; Huerre and Monkewitz, 1990), we introduce the Green's function for a pulse perturbation in one spatial dimension $G(y, t)$ defined by

$$
\mathcal{L} G(y, t)=\delta(y) \delta(t)
$$

where $\mathcal{L}$ is the linear operator and $\delta$ denotes the Dirac delta function. The flow can be classified to four categories depending on the types of the impulse response: linearly 
stable, linearly unstable, convectively unstable and absolutely unstable.

A system is linearly stable if

$$
\lim _{t \rightarrow \infty} G(y, t)=0 \text { along all rays } y / t=\text { constant }
$$

and linearly unstable if

$$
\lim _{t \rightarrow \infty} G(y, t)=\infty \text { along at least one ray } y / t=\text { constant. }
$$

Given an unstable flow, it is convectively unstable if

$$
\lim _{t \rightarrow \infty} G(y, t)=0 \text { along the ray } y / t=0
$$

absolute unstable if

$$
\lim _{t \rightarrow \infty} G(y, t)=\infty \text { along the ray } y / t=0 .
$$

Thus absolute and convective instability are defined based on the impulse response at a fixed location, say $y_{0}$. After a sufficiently long time, the ray $y / t=0$ refers to the zero group velocity so the corresponding response is local. For an unstable flow, if the local response to a pulse perturbation is amplifying, then the flow is called absolutely unstable, otherwise the pulse perturbation will fade away from $y_{0}$ for a sufficient long time, and such unstable flow is called convectively unstable. Figure 4-2 shows an illustration of the concept. Besides $y / t \rightarrow 0$, another important ray is

$$
\frac{y}{t}=\left.\frac{\partial \Omega_{r}}{\partial \ell_{r}}\right|_{\ell_{m}}=\left.c_{g}\right|_{\ell_{m}}
$$

where $\ell_{m}$ is the wavenumber corresponding to the most unstable mode satisfying

$$
\frac{\partial \Omega_{i}}{\partial \ell_{r}}\left(\ell_{m}\right)=0
$$




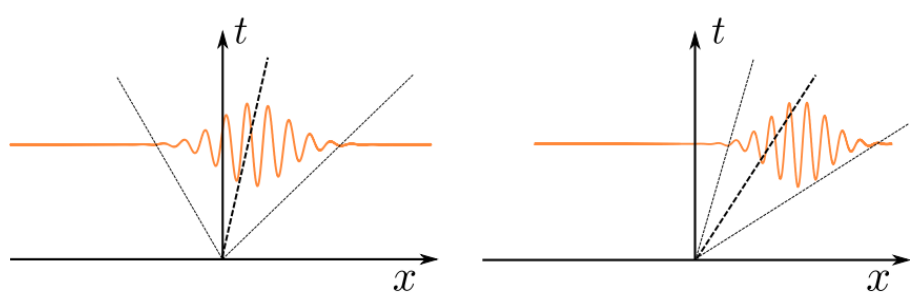

Figure 4-2: The sketch of the absolute and convective instability, which was first illustrated in Briggs (1964). The thick dashed line represents the ray of the most unstable mode.

This is the ray of the most unstable mode.

The distinction between notions of the absolute instability and the spatial instability is more important for the spatially varying flows than for parallel flows. For parallel flows, the response of the initial impulse will eventually be dominated by the wave packet related to $\ell_{m}$ traveling in the speed $\left.c_{g}\right|_{\ell_{m}}$. In other words, the stability problem for a parallel flow is invariant under the Galilean group, meaning the growth rates are not affected by a constant flow shift. However, this invariance is broken for a spatially varying flow. The flow could be more unstable over one region but becomes less unstable over other regions. In a flow that is convectively unstable everywhere, no global mode can be formed because of the absence of absolute instability. The signal is traveling and constantly swept away from a fixed location. If the flow is non-parallel and is absolutely unstable over certain regions, the growth rate of what was once the tail can dominate the former peak to become the maximum amplitude of the mode. A global mode then emerges.

\subsection{Formulation}

We consider a system with a basic state consisting of a meridional boundary current and a large-scale flow.

$$
\bar{\psi}=\bar{\psi}_{1}(x)+\bar{\psi}_{2}(x, y)
$$


where $\bar{\psi}$ represents the stream function of the total basic state, $\bar{\psi}_{1}$ represents the boundary current and $\bar{\psi}_{2}$ represents the large-scale flow. Correspondingly, $\bar{v}_{1}$ and $\left(\bar{u}_{2}, \bar{v}_{2}\right)$ denote the velocity fields of the boundary current and the large-scale flow. Here we focus on the stability of an eastern boundary current. The current is represented by a half Bickley profile $\bar{\psi}_{1}=-\int_{x} \operatorname{sech}^{2}(x) d x$ within a semi-infinite domain $-\infty<x \leq 0$ where a wall is located at $x=0$. Two different large-scale flows are considered and discussed in the following.

\subsubsection{Large scale flow, a partial-gyre}

To simplify the problem but still retain its essential parts, we define a partial-gyre flow to consist of a weak uniform zonal flow in the interior with an amplitude $\bar{U}_{2}$ and an inertial eastern boundary layer with a width $L_{I}$,

$$
\begin{aligned}
\bar{\psi}_{2}(x, y)=-\bar{U}_{2} y \quad ; \quad x \leq-L_{I} \\
J\left(\bar{\psi}_{2}, \nabla^{2} \bar{\psi}_{2}+\beta y\right)=0 \quad ; \quad-L_{I}<x<0
\end{aligned}
$$

with boundary conditions

$$
\begin{aligned}
-\bar{\psi}_{2 y} & =\bar{U}_{2} \text { at } x=-L_{I} \\
\bar{\psi}_{2 x} & =0 \text { at } x=-L_{I} \\
\bar{\psi}_{2} & =0 \text { at } x=0 .
\end{aligned}
$$

The $J()=0$ implies $\nabla^{2} \bar{\psi}_{2}+\beta y$ is a function of $\bar{\psi}_{2}$. We can examine the zonal structure of the large-scale flow by first permitting $-L_{I} \rightarrow-\infty$, then matching the interior solution which is $\bar{\psi}_{2}=-\bar{U}_{2} y$. The resulting equation is

$$
\partial_{x x} \bar{\psi}_{2}+\beta y=-\beta \bar{\psi}_{2} / \bar{U}_{2}
$$

The exact solution can be found by assuming $\bar{\psi}_{2}=-F(x) y$. We get 


$$
F=\bar{U}_{2}-\bar{U}_{2} e^{ \pm \sqrt{-\beta / \bar{U}_{2}} x}
$$

When $\bar{U}_{2}>0$ it is a wave solution, and $\bar{U}_{2}<0$ it is an exponentially decaying solution. Figure 4-3 shows an example of the solution of $\mathrm{F}$ with different $\bar{U}_{2}$.

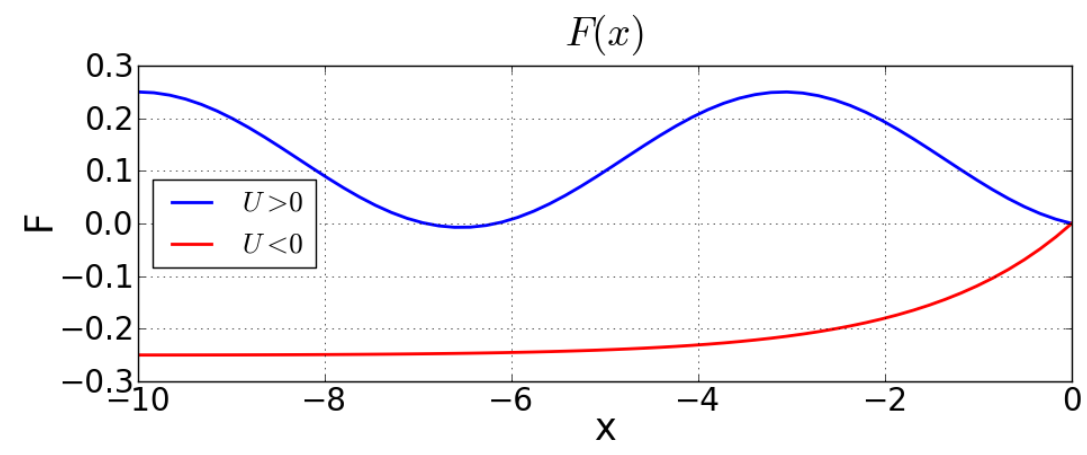

Figure 4-3: The solution of $F$ with $\beta=0.1$ for $\bar{U}_{2}=-0.25$ (red) and $\bar{U}_{2}=0.25$ (blue).

If we keep $L_{I}$ finite, the boundary layer is simplified to the third order ODE

$$
F^{\prime \prime \prime} F-F^{\prime \prime} F^{\prime}+\beta F^{\prime}=0
$$

where ' represents the partial derivative in $x$. The boundary conditions become

$$
\begin{aligned}
F & =\bar{U}_{2} \quad x=-L_{I} \\
F^{\prime} & =0 x=-L_{I} \\
F & =0 x=0,
\end{aligned}
$$

which mean that the zonal velocity is $\bar{U}_{2}$ in the interior, the meridional velocity is 0 over the region $x<-L_{I}$, and the no normal flow condition at the wall $x=0$.

The above boundary conditions render an eigenvalue problem, whose solutions essentially share the same features as the solutions in equation 4.20. The inertial boundary layer with the above boundary conditions can be solved numerically. However, for a practical purpose, we choose $\bar{U}_{2}>0$, and the flow has a structure of $1 / 4$ 


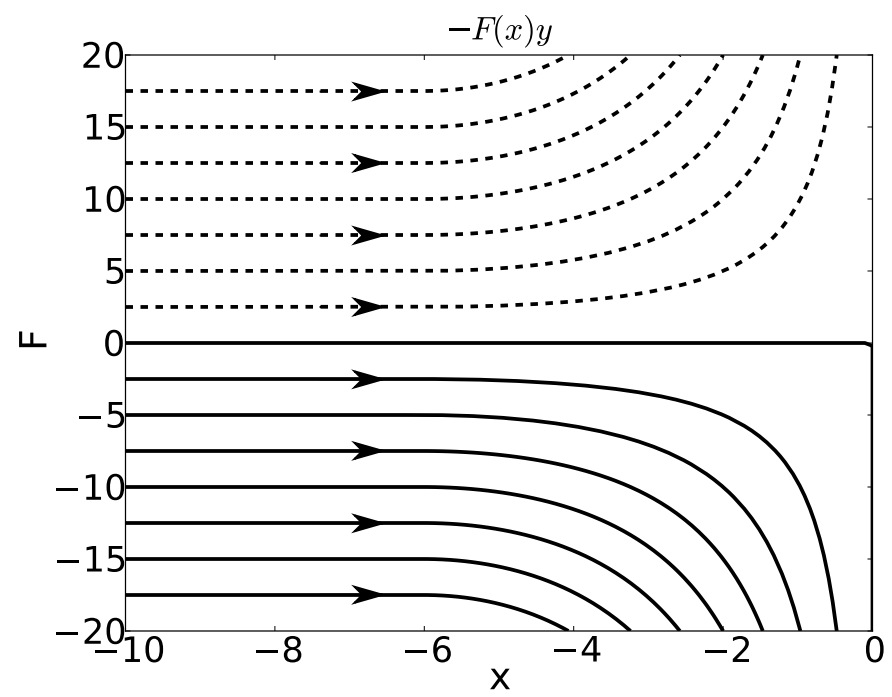

Figure 4-4: An example of the two dimensional structure of the large-scale flow. The parameters are $L_{I}=-6, W_{x}=10$ and $W_{y}=40$. The arrows indicate the flow direction.

of one sinusoidal wave. We should notice that $\bar{U}_{2}<0$ is also relevant for large-scale flows. It is not studied here but worth a future investigation. $W_{x}$ and $W_{y}$ are used hereafter to represent the zonal and meridional extent of a model domain. Figure 4-4 shows an example of the two dimensional structure of the partial-gyre flow. The function representing the zonal structure becomes

$$
F= \begin{cases}-\bar{U}_{2} \sin \left(\frac{\pi x}{2 L_{I}}\right), & \text { at }-L_{I}<x \leq 0 \\ \bar{U}_{2}, & \text { at } x \leq-L_{I} .\end{cases}
$$

The barotropic QG equation for the PV perturbation $q$ is

$$
\partial_{t} q+J(\bar{\psi}, q)+J(\psi, Q)=0
$$


where

$$
\begin{aligned}
\bar{\psi} & =-F(x) y+\int \bar{v}_{1}(x) d x \\
Q & =\nabla^{2} \bar{\psi}+\beta y=-F_{x x} y+\bar{v}_{1 x}+\beta y \\
q & =\nabla^{2} \psi \\
J(\bar{\psi}, q) & =\left(F \partial_{x}+\left(\bar{v}_{1}-F_{x} y\right) \partial_{y}\right) \nabla^{2} \psi \\
J(\psi, Q) & =\left(\left(\beta-F_{x x}\right) \partial_{x}+\left(F_{x x x} y-\bar{v}_{1 x x}\right) \partial_{y}\right) \psi
\end{aligned}
$$

and the subscript $x$ represents the $x$-derivative.

The perturbation equation with a large-scale flow $\bar{\psi}_{2}(x, y)=-F(x) y$ becomes

$$
\begin{aligned}
\mathcal{L} \psi= & 0 \\
\mathcal{L}= & {\left[\partial_{t}+F \partial_{x}+\left(\bar{v}_{1}-F_{x} y\right) \partial_{y}\right] \nabla^{2} } \\
& +\left(\beta-F_{x x}\right) \partial_{x}-\left(\bar{v}_{1 x x}-F_{x x x} y\right) \partial_{y} .
\end{aligned}
$$

This equation shows several terms which involve the background velocity and vorticity field. $F \partial_{x}$ represents the zonal transport of the perturbation PV by the large-scale flow, which is absent in the case with a parallel meridional jet. $\left(\bar{v}_{1}-F_{x} y\right)$ is the total meridional velocity of the large-scale flow and the boundary current. $F_{x x}$ contributes to the meridional gradient of the background $\mathrm{PV}$, which acts in the same way as does the planetary $\beta . F_{x x x} y$ is the zonal PV gradient of the largescale flow. This equation is solved in the next section using the WKB method and time-dependent numerical simulation.

\subsubsection{Large scale flow, a double-gyre}

We add more complexity into the problem by replacing the partial-gyre flow with a double-gyre structure. The partial-gyre flow has a simple structure which is linear in $y$. It significantly simplifies the background-flow-influenced stability equation. The north-south boundary, however, is technically not periodic for a system with a partial- 
gyre flow. Because the nonlinear simulations will be used later in thenext chapter, a double-gyre structure becomes more feasible for long time integration. However, one should be cautious in interpreting results of the longtime integration because the real ocean is not north-south periodic. In this chapter, the behavior of the doublegyre-influenced linear stability equation is investigated using a linear time-dependent numerical model. The results validate the mechanism shown in an analysis using WKB method, and also suggests behaviors which might exist in a nonlinear regime. More details about the specific model configuration are discussed in each section.

\subsection{WKB solution for the partial-gyre flow}

The WKB method is used to simplify the stability equation with a partial-gyre flow to an eigenvalue problem. The stability problem becomes two-dimensional if the mean state varies both in $x$ and $y$, and the normal modes are no longer solutions. However, as we assume the large-scale flow is much weaker and broader than the jet, the variation of the basic state is much slower in the streamwise (meridional) direction than in the cross-stream (zonal) direction. There are two distinct spatial scales. We can introduce a small parameter based on the spatial aspect ratio into the equation and look for the local WKB solutions.

\subsubsection{Formulation}

Define $y_{1}=\epsilon y$, where $\epsilon=L_{j e t} / L_{y}$ is the spatial ratio, and $L_{j e t}, L_{y}$ represent the scale of the basic state (the boundary current and the large-scale flow) in cross-stream and streamwise direction respectively, the WKB solution of the perturbation can be written as

$$
\psi=\phi\left(x, y_{1}\right) e^{\frac{i S\left(y_{1}\right)}{\epsilon}} e^{i \omega t}
$$


For modes with this form, their associated spatial derivatives are (omitting $e^{i \omega t}$ )

$$
\begin{aligned}
\partial_{y} \psi & =\left(i S^{\prime} \phi+\epsilon \psi^{\prime}\right) e^{i S / \epsilon} \\
\partial_{y y} \psi & =\left(-S^{\prime 2} \phi+i \epsilon\left(S^{\prime \prime} \phi+2 S^{\prime} \phi^{\prime}\right)\right) e^{i S / \epsilon}+\mathcal{O}\left(\epsilon^{2}\right) \\
\partial_{y y y} \psi & =\left(-i S^{\prime 3} \phi-3 \epsilon S^{\prime}\left(S^{\prime \prime} \phi+S^{\prime} \phi\right)\right) e^{i S / \epsilon}+\mathcal{O}\left(\epsilon^{2}\right) \\
\partial_{x} \psi & =\phi_{x} e^{i S / \epsilon} \\
\partial_{x x} \psi & =\phi_{x x} e^{i S / \epsilon} \\
\partial_{y x x} \psi & =\left(i S^{\prime} \phi_{x x}+\epsilon \phi_{x x}^{\prime}\right) e^{i S / \epsilon} \\
\partial_{x y y} \psi & =\left(-S^{\prime 2} \phi_{x}+i \epsilon\left(S^{\prime \prime} \phi_{x}+2 S^{\prime} \phi_{x}^{\prime}\right)\right) e^{i S / \epsilon}+\mathcal{O}\left(\epsilon^{2}\right)
\end{aligned}
$$

where ' represents the derivative in $y_{1}$ and subscript $x$ represents the derivative in $x$. The perturbation equation 4.32 becomes

$$
\begin{array}{r}
i \omega\left[\phi_{x x}-S^{\prime 2} \phi+i \epsilon\left(S^{\prime \prime} \phi+2 S^{\prime} \phi^{\prime}\right)\right]+F \phi_{x x x}+F\left[-S^{\prime 2} \phi_{x}+i \epsilon\left(S^{\prime \prime} \phi_{x}+2 S^{\prime} \phi_{x}^{\prime}\right)\right] \\
+\left(\bar{v}_{1}-F_{x} y\right)\left(i S^{\prime} \phi_{x x}+\epsilon \phi_{x x}^{\prime}\right)+\left(\bar{v}_{1}-F_{x} y\right)\left[-i S^{\prime 3} \phi-3 \epsilon S^{\prime}\left(S^{\prime \prime} \phi+S^{\prime} \phi\right)\right] \\
+\left(\beta-F_{x x}\right) \phi_{x}-\left(\bar{v}_{1 x x}-F_{x x x} y\right)\left(i S^{\prime} \phi+\epsilon \phi^{\prime}\right)+\mathcal{O}\left(\epsilon^{2}\right)=0 .
\end{array}
$$

Its $\mathcal{O}(0)$ equation is :

$$
\begin{aligned}
-i \omega\left(\phi_{x x}-\right. & \left.S^{\prime 2} \phi\right)+F \phi_{x x x}-F S^{\prime 2} \phi_{x}+\left(\bar{v}_{1}-F_{x} y\right)\left(i S^{\prime} \phi_{x x}\right) \\
& +\left(\bar{v}_{1}-F_{x} y\right)\left(-i S^{\prime 3} \phi\right)+\left(\beta-F_{x x}\right) \phi_{x}-\left(\bar{v}_{1 x x}-F_{x x x} y\right)\left(i S^{\prime} \phi\right)=0 .
\end{aligned}
$$

As the large-scale flow is weak, the zonal variation of $F$ is much smaller than that of the jet. Therefore we introduce a new zonal scale $x_{1}=\epsilon x$ for $F$. Then $F_{x}=\epsilon F_{x_{1}}$, and $\bar{v}_{1}-F_{x} y=\bar{v}_{1}-F_{x_{1}} y_{1}, F_{x x} \sim \mathcal{O}\left(\epsilon^{2}\right), F_{x x x} \sim \mathcal{O}\left(\epsilon^{3}\right)$. The $\mathcal{O}(0)$ equation becomes

$$
\begin{aligned}
& F \phi_{x x x}+i\left[\ell\left(\bar{v}_{1}-F_{x_{1}} y_{1}\right)-\omega\right] \phi_{x x}+ \\
& \quad\left(\beta-F \ell^{2}\right) \phi_{x}+i \ell\left[\ell \omega-\ell^{2}\left(\bar{v}_{1}-F_{x_{1}} y_{1}\right)-\bar{v}_{1 x x}\right] \phi=0,
\end{aligned}
$$


where $\ell$ represents $S^{\prime}$. We can get the familiar stability equation for the parallel merdional jet by setting $F=0$

$$
i\left(\ell \bar{v}_{1}-\omega\right) \phi_{x x}+\beta \phi_{x}+i \ell\left(\ell \omega-\ell^{2} \bar{v}_{1}-\bar{v}_{1 x x}\right) \phi=0
$$

in which $\omega=c \ell$. In the temporal stability problem, we substitute $\omega=c \ell$ into (4.46) and seek the complex $c$ for a real $\ell$. Again, here we focus on the spatial instability as we are more interested in a individual wave packet behavior, and choose $\omega$ to be real and $\ell$ to be complex in order to get the spatial structure of the stability of the nonparallel jet. This equation is solved using the shooting method, as in Chapter 2. Notice that $F=0$ at $x=0$, where the equation becomes singular. Solutions over that point could be found by the Frobenius method. Here we avoid the analytical solution and use an approximate numerical solution. Define a small interval $\delta x$, and assume $F=0$ between $x=-\delta x$ and the boundary $x=0$. At $x=-\delta x$, two solutions are computed. One is calculated by integrating the full equation (4.45) from the interior, and another is calculated by integrating (4.46) from $x=0$. The differences in $\psi$ and $\psi_{x}$ between those two solutions form the discrepancy function. The eigenvalues are found by minimizing this discrepancy.

Before discussing the numerical eigenvalue solutions, we first examine the differences between (4.45) and (4.46). Every term with $F$ in (4.45) is due to the large-scale flow. From (4.32) we see that the influence of the large-scale flow enters every term of $J(\bar{\psi}, q)$ and $J(\psi, Q)$.

In the leading order WKB equation, there are two influences from the largescale flow. The first is related to the zonal transport of the perturbation PV by the large-scale flow, represented by $F \phi_{x x x}$ and $-F \ell^{2} \phi_{x}$ which are derived from the term $\bar{u}_{2} \partial_{x} \nabla^{2} \psi$. Another influence is related to the meridional advection of perturbation PV by the large-scale flow, represented by $-F_{x_{1}} y_{1}$ in the term $V-F_{x_{1}} y_{1}$. The advection of the background PV field by the perturbation velocity is secondary, again because the large-scale flow has much larger spatial scale than the perturbations. 
The first influence, the zonal advection, can be separated by examining the solution at $y_{1}=0$, where the meridional velocity of the large-scale flow is zero. The stability equation 4.45 at $y_{1}=0$ becomes

$$
F \phi_{x x x}+i\left(\ell \bar{v}_{1}-\omega\right) \phi_{x x}+\left(\beta-F \ell^{2}\right) \phi_{x}+i \ell\left(\ell \omega-\ell^{2} \bar{v}_{1}-\bar{v}_{1 x x}\right) \phi=0
$$

In equation 4.47 , the zonal advection of the perturbation $\mathrm{PV},-F \ell^{2} \phi_{x}$, is grouped together with $\beta$, meaning that they have similar effect. In a more intuitive sense, both the zonal advection and $\beta$ can generate zonal waves from localized oscillations, which may be induced by instabilities. The $\beta$ effect generates Rossby waves, and zonal advection generates wave-like patterns by zonally advecting oscillatory perturbations. The consistency between the zonal advection and beta effect can be checked by their signs. The zonal velocity of the large-scale flow is represented by $\bar{u}_{2}=-\partial_{y} \bar{\psi}_{2}=$ $F$. For a westward zonal flow that generates westward propagating features, $F$ is negative, and $-F \ell^{2}$ is positive. The positive $-F \ell^{2}$ has the same sign as that of $\beta$ that generates westward propagating Rossby waves. From the previous chapter, we know that positive $\beta$ has a stabilizing effect on the eastern boundary current with a half Bickley profile, so that the term $-F \ell^{2} \partial_{x}$ is negative and has a destabilizing effect for an eastward inflow where $F>0$. We call $\beta-F \ell^{2}$ the effective $\beta$ henceforth. The influence of the zonal advection is investigated by varying the amplitude of $F$. The influence is a mixture of the destabilizing effect by $-F \ell^{2} \phi_{x}$ and the stabilizing effect by $F \phi_{x x x}$ which is discussed in Section 4.4.2.

The second influence, meridional advection, is studied by examining solutions at different $y_{1}$ with a fixed large-scale flow intensity $F$. Results are discussed in the following.

\subsubsection{Results}

Given a real frequency $\omega$ and $\beta$, two complex meridional wavenumber solutions corresponding to the two branches of $l$ exist for the above spatial instability problem 
without a large-scale flow. Only one of the solutions is physically meaningful. The branch picking is usually a complicated process, but it is simple in our case because we only consider the region around the stagnation point. Briggs' rule states that the growth rate of an amplifying mode changes sign following increase of the temporal growth rate of the forcing. This means that if we calculate the complex eigenvalue $\ell$ with a complex frequency $\omega=\omega_{r}+i \omega_{i}$, then $\ell_{i}$ will change sign following the increase of $\omega_{i}$. Figure 4-5 shows that the $\ell_{i}$ that changes sign is positive at $\omega_{i}=0$, so it is the solution that represents the amplifying mode. Therefore, the solutions with negative $\ell_{i}$ represent evanescent modes and are not discussed here. The positive $\ell_{i}$ represents a southward amplifying structure, $e^{-\ell_{i} y}$, which is the structure for an amplified wave with a southward group velocity in the convectively unstable boundary current considered here.

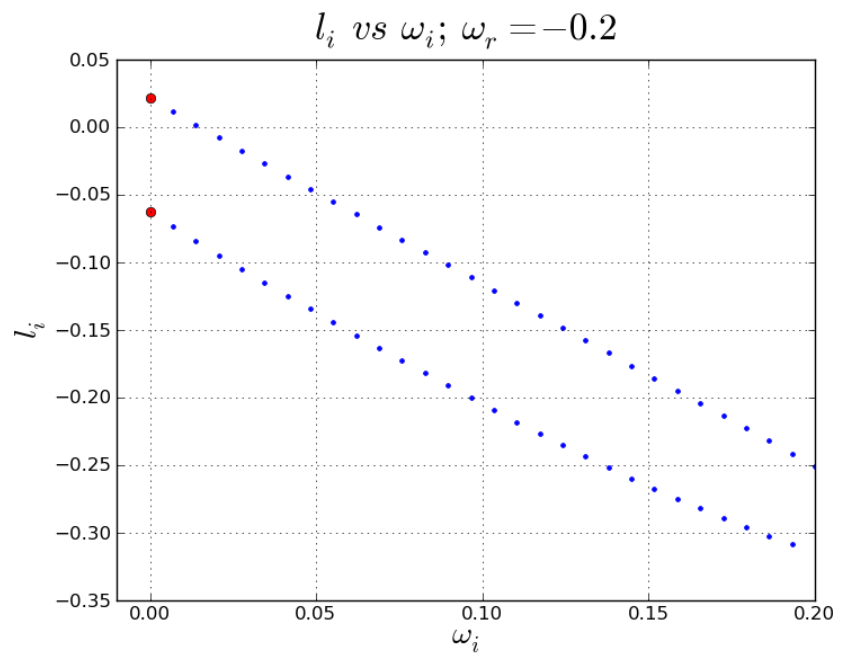

Figure 4-5: $\ell_{i}$ vs $\omega_{i}^{f}$ with $\beta=0.07$. Two dotted lines represent two solutions for each $\omega_{i}^{f}$. The two red circles are the solutions with $\omega_{i}^{f}=0$. The solution which changes sign following the increase of $\omega_{i}^{f}$ is the one with a positive value at $\omega_{i}^{f}=0$.

\section{The influence of zonal PV advection}

The influence of the zonal PV advection by the large-scale flow on the spatial stability becomes evident at $y_{1}=0$ where the adjustment of the meridional velocity by the 
large-scale flow is zero so that the Doppler effect is eliminated in equation 4.47. We discuss three properties of the instability: the spatial growth rate $\ell_{i}$, the phase speed $c_{r}$ and the zonal wavenumber $k$. They are calculated as functions of the intensity of the large-scale flow, i.e. the velocity scale $\bar{U}_{2}$ of the inflow $-F(x) y$ at $x=-L_{I}$. The results show that an increase in the large-scale flow intensity leads to a decrease of the spatial growth and an increase of the meridional phase speed (Figure 4-6).

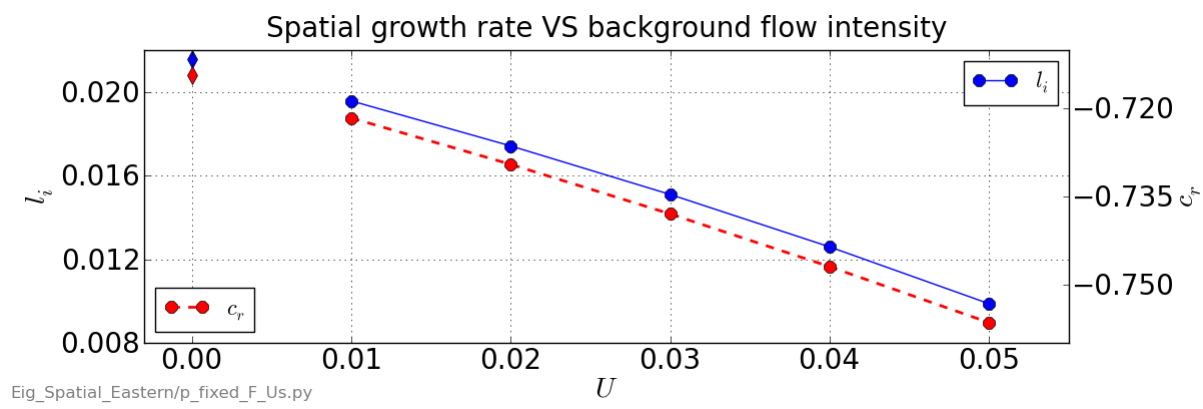

Figure 4-6: The influence of the zonal PV advection is shown by $\ell_{i}$ as a function of $\bar{U}_{2}$ calculated at $y=0$. The values for the meridional jet without any large-scale flow influence are indicated by the diamond-symbols. The red line shows $c_{r}$, and the blue line shows $\ell_{i}$. The overall effect of the zonal PV advection is that $\ell_{i}\left(c_{r}\right)$ decreases (increases) following the increase of the inflow intensity. The parameters used in the calculation are $\beta=0.07, \omega=-0.2$.

First we discuss the influence of the large-scale flow on $\ell_{i}$. There are two terms in the zonal advection of $\mathrm{PV},-F \ell^{2} \phi_{x}$ and $F \phi_{x x x}$. The first term comes from $-\bar{u} u_{y y}$ and has a destabilizing influence. The overall effect of these two terms are stabilizing, which is show in Figure 4-6. As a result, the second term has the stabilizing effect. We gain more insights by examining the contribution of the term $F \phi_{x x x}$ in the energy budget. By multiplying the complex conjugate of $\phi$ on both sides of the stability equation, we get its energy contribution, $\phi^{*} F(x) \phi_{x x x}$ where $*$ represents the complex conjugate. To simplify the problem, we take the asymptotic form of $F(x)$ around 
$x=0$ which is $F(x)=\bar{U}_{2} x$. The $\phi^{*} F(x) \phi_{x x x}$ is rewritten in the following form

$$
\begin{aligned}
\phi^{*}\left(\bar{U}_{2} x \phi_{x x x}\right) & =S_{x}+\frac{3}{2} \bar{U}_{2}\left|\phi_{x}\right|^{2} \\
S & =\bar{U}_{2}\left(x \phi \phi_{x x}-\frac{1}{2}|\phi|_{x}^{2}-\frac{x}{2}\left|\phi_{x}\right|^{2}\right)
\end{aligned}
$$

The second term in equation 4.48 is always positive and indicates the stabilizing effect (remember the energy tendency is $-\phi^{*}\left(x \phi_{x x x}\right)$ ). The contribution from the divergent term is zero for the trapped modes. For the radiating mode in an unbounded domain, the argument based on the energy budget becomes delicate because the change of the large-scale circulation can cause the change of the energy radiation.

Figure 4-6 shows that increasing the zonal onshore flow decreases the spatial growth rate. The influence of the zonal PV advection by the large-scale flow has a stabilizing effect. Because the zonal velocity is independent of $y$ in the partial-gyre flow, we expect the influence of the zonal velocity by the large-scale flow is the same for all stations along streamwise direction.

Next the influence of the zonal PV advection on the meridional phase speed $c_{r}$ is discussed. In the previous chapter, we discovered that increasing $\beta$ reduces the phase speed of the perturbation in an eastern boundary jet. Here we show that the zonal PV advection contributes to the $\beta$ effect as indicated by the effective $\beta$ term: $\beta-F \ell^{2} \phi_{x}$. As $-F \ell^{2} \phi_{x}$ is negative, it reduces the effective $\beta$, so that the phase speed increases. The red line in Figure 4-6 shows that the phase speed increases following the increase of the zonal flow intensity. This phase speed increase is due to the equivalent $\beta$ effect, which is different than the meridional velocity adjustment as discussed in the next section.

What happens to the zonal structure, i.e., the zonal wavenumber? We know that there is a westward radiating boundary condition in the interior, where the meridional velocity is zero and the wave characteristics satisfy the dispersion relation of a Rossby wave in a zonal flow. Given a fixed frequency, wavenumbers change according to the 
dispersion relation.

$$
\omega=\bar{U}_{2} k-\frac{\beta k}{k^{2}+\ell^{2}}
$$

where $k=k_{r}+\mathrm{i} k_{i}$ and $\ell=\ell_{r}+\mathrm{i} \ell_{i}$. Both $k_{r}$ and $k_{i}$ decrease following the increase of $\bar{U}_{2}$ as shown in Figure 4-7 in terms of the absolute value, which means that the stronger large-scale flow leads to the larger zonal wave and the slower decay rate. In the previous chapter, it is suggested that $\left|k_{r}\right|>\left|k_{i}\right|$ and the wave speed satisfies the Rossby wave dispersion relation for an unstable mode to radiate. Here we noticed that $\left|k_{r}\right|<\left|k_{i}\right|$ and the phase speeds do not satisfy the dispersion relation, so that an unstable perturbation with $\omega_{r}=-0.2$ is not radiating. Other frequencies may be associated with radiating perturbations.

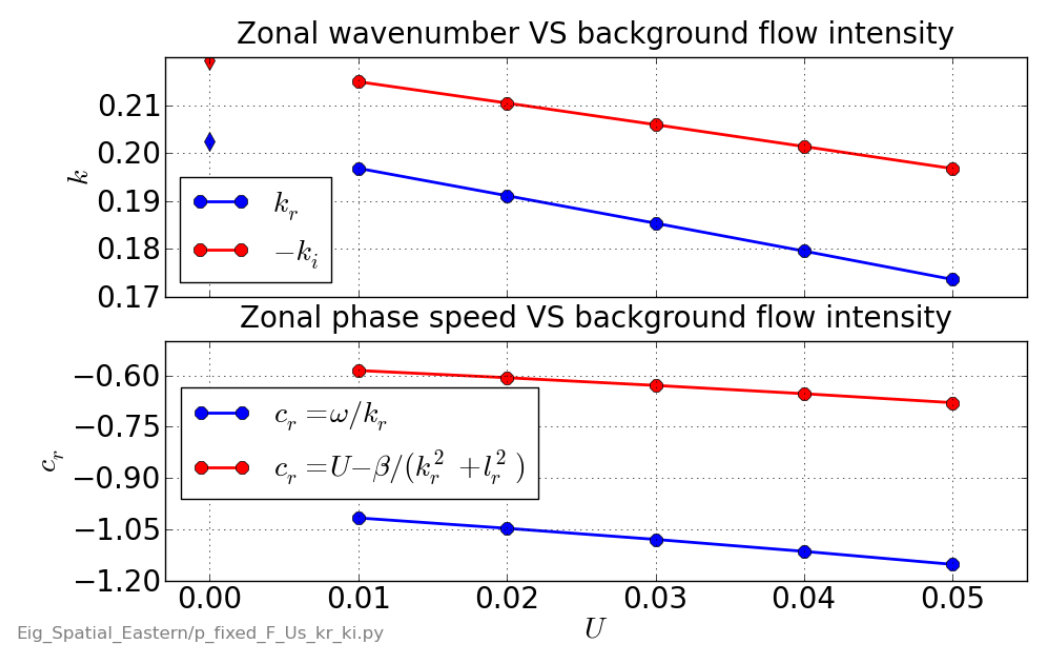

Figure 4-7: The zonal wavenumber $k_{r}$ (upper) and $k_{i}$ (bottom) as a function of $Y$.

\section{The influence of the meridional velocity adjustment}

The influence of the meridional PV advection by the large-scale flow is done through modifying the jet structure and the jet speed to $\bar{v}_{1}-\frac{d F\left(x_{1}\right)}{d x_{1}} y_{1} . F$ is a function of $x$, so that $\frac{d F\left(x_{1}\right)}{d x_{1}} y_{1}$ modifies the Bickley jet zonal structure which changes the stability characteristics. Another effect of $-\frac{d F\left(x_{1}\right)}{d x_{1}} y_{1}$ is to accelerate the flow along the boundary. In the WKB formulation, we see the PV contribution of the large-scale flow to 
the problem is small, so that the effect of the modification of the zonal structure of the Bickley jet is small (also confirmed later). Then the dominating influence of the meridional advection of the PV perturbation by the large-scale flow is through the adjustment of the meridional velocity of the boundary current. The large-scale flow can either accelerate the jet (over $y_{1}>0$ ) or retard the jet (over $y_{1}<0$ ). We verify this assertion as follows.

The spatial growth rate $\ell_{i}$ in the stability equation 4.45 is calculated for each $y_{1}$. Figure 4-8 shows the spatial growth rate $\ell_{i}$ as a function of $y_{1}$. The increase of the intensity of the large-scale flow stabilizes the jet (from the black line to the blue line and then the green line). The influence of the zonal PV advection is indicated by the difference between black solid lines and dashed lines, and is uniform for all locations given a fixed $\bar{U}_{2}$. The sloping feature in the spatial growth rates as a function of $y_{1}$ is due to the influence of the meridional velocity adjustment. The slope becomes steeper when $\bar{U}_{2}$ becomes larger.

By using a Galilean transform, we can easily prove that if $c=c_{r}+i c_{i}$ is an eigenvalue of the stability equation with a Bickley profile $\bar{v}_{1}(x)$, then $c=(C+$ $\left.c_{r}\right)+i c_{i}$ is an eigenvalue for the stability equation with a velocity profile $\bar{v}_{1}(x)+C$, where $C$ is an arbitrary constant. In other words, superposing a constant meridional velocity field to the Bickley jet profile gives the same temporal growth rate but a increased/decreased phase speed. Also, the new group velocity becomes $c_{g}+C$.

Even though it does not change the temporal growth rate, an altered group velocity can change the spatial growth rate $\ell_{i}$ by the relationship $\ell_{i}=\omega_{i} / c_{g}$ given a small $\omega_{i}$. This meridional velocity adjustment dominates in the influence of the meridional PV advection, which can be confirmed by comparing two spatial growth rates calculated by two methods. The first one is calculated by directly solving the eigenvalue problem (4.45). The second one is derived from the eigen-solutions of the temporal instability with the jet profile $\bar{v}_{1}(x)+C$ where $C$ is the phase speed shift and is calculated from the eigenvalues of the spatial instability. In the first one, the mean state consists of the Bickley jet profile and $\partial_{x_{1}} F y_{1}$ (note that $F$ is also a function of $x$ ), while in the 
second one the mean state is the Bickley jet plus a constant velocity. If the two spatial growth rates approximately agree with each other, then we can confirm that the effect of the meridional velocity adjustment dominates in the meridional PV advection.

We calculate the two solutions in the following order. First we solve the spatial instability equation 4.45 for different locations $y_{1}$ given a real frequency, say $\omega_{r 0}$, to get the eigenvalues $\ell_{r 0}+i \ell_{i 0}$ as a function of $y_{1}$. The derived phase speed at $y_{1}$ is $\left.c\right|_{y_{1}}=\omega_{r 0} /\left.\ell_{r 0}\right|_{y_{1}}$. The phase speed shift $C_{s h i f t}$ for different $y_{1}$ is calculated by subtracting the phase speed at $y_{1}=0:\left.C_{s h i f t}\right|_{y_{1}}=\left.c\right|_{y_{1}}-\left.c\right|_{y_{1}=0}$. With a known $\left.\ell_{r 0}\right|_{y_{1}}$ and $\left.C_{\text {shift }}\right|_{y_{1}}$, the temporal growth rate $\left.c_{i 0}\right|_{y_{1}}$ is calculated for a boundary current with a velocity profile $\bar{v}_{1}=-\operatorname{sech}^{2}(x)+\left.C_{\text {shift }}\right|_{y_{1}}$ at different $y_{1}$. The spatial growth rate is then derived by $\left.\ell_{i}\right|_{y_{1}}=\left.\left(\omega_{i} / c_{g}\right)\right|_{y_{1}}$, where $c_{g}$ is numerically calculated using $c_{g}=\Delta \omega_{r} / \Delta \ell_{r}$. We can confirm that the velocity adjustment effect dominates in the influence of the meridional PV advection if these two solutions agree with each other.

Figure 4-8 shows the result. In the bottom panel, the velocity adjustment on the phase speed is clear. The perturbation is accelerated downstream but decelerated upstream. The discrepancies among different cases at $y_{1}=0$ is due to the accelerating effect by the zonal PV advection. Correspondingly, the wavenumber changes according to the relation $c_{r}=\omega_{r} / \ell_{r}$ for a fixed $\omega_{r}$. The wavelength becomes longer downstream than upstream. The spatial growth rate $\ell_{i}$ is reconstructed using the information in $\ell_{r}, c_{r}$, and the temporal eigenvalue solution. The red dash-cross line shows the derived values. An adjustment is applied to make two solutions equal at $y_{1}=0$ in order to eliminate the effect of the zonal PV advection. After the adjustment, the two spatial growth rates agree very well with each other. This demonstrates that the velocity adjustment is the major process in the influence of the meridional PV advection.

As for the zonal structure, Figure 4-9 shows the zonal wavenumber as a function of $y_{1}$. The discrepancies between black lines are caused by the influence of the zonal PV advection, and the slope is due to the meridional PV advection. The zonal 

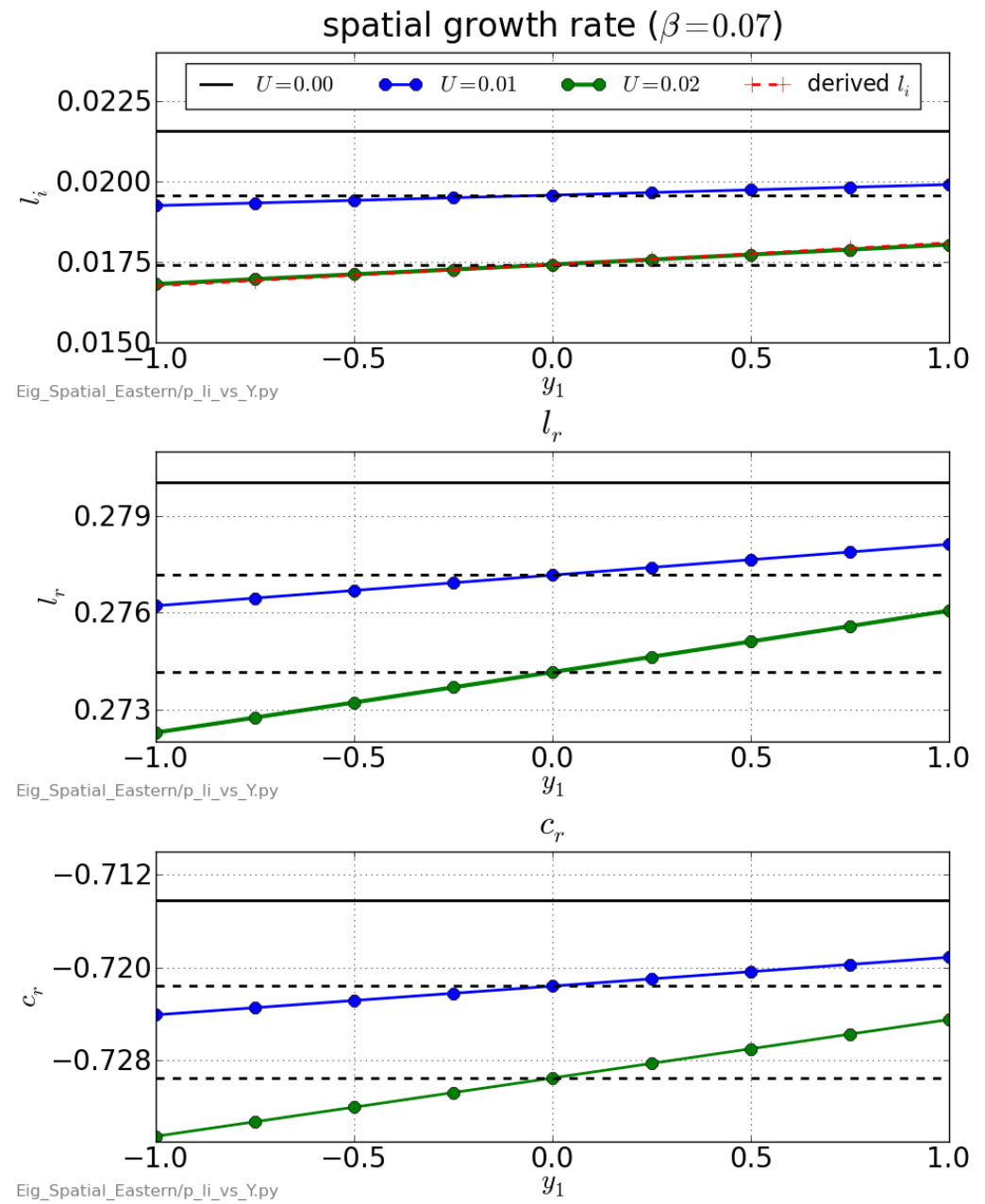

Figure 4-8: The spatial growth rate, $\ell_{i}$, as a function of $y_{1}$. By comparing the blue line and the green line, we conclude that increasing the large-scale flow decreases the spatial growth rate. By comparing the green line and the red-dashed line, we see that increasing $\beta$ also decreases the spatial growth rate for all modes. The solid black lines are for the case without large-scale flow influence. The dashed black lines are plotted for reference. 
wavenumber $k_{r}$ becomes smaller, and the decay rates $k_{i}$ also becomes smaller in the downstream direction. It is noteworthy that the real frequency $\omega=-0.2$ corresponds to a trapped mode, because the westward zonal decay scale $\left(1 / k_{i}\right)$ is shorter than the zonal wavelength $\left(1 / k_{r}\right)$. This criteria is discussed in Chapter 2 . It would be interesting to explore the system's response to a frequency that corresponds to a radiating mode. A further study is needed.
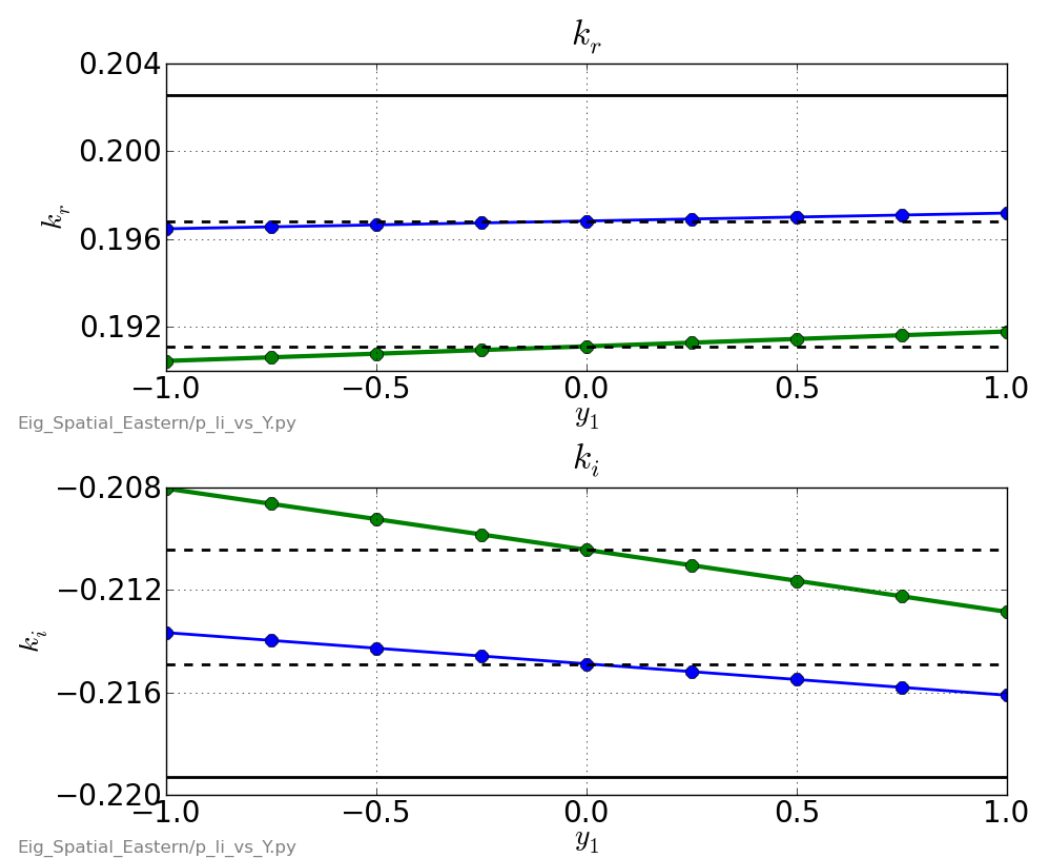

Figure 4-9: The zonal wavenumber $k_{r}$ (upper) and $k_{i}$ (bottom) as a function of $y$. The blue and green line represent the cases with $U=0.01$ and $U=0.02$, respectively.

\subsubsection{Summary}

Using the WKB method, we analyze the stability of the boundary current modified by a large-scale flow with a focus on the leading order behavior. The partial-gyre flow consists of a uniform zonal flow in the interior and a diffluent structure over the boundary region. Its influence can be decomposed into two terms. The first one is related to the zonal PV advection by the large-scale flow, which has an overall stabilizing effect. The second one is related to the meridional PV advection by the 
large-scale flow, which produces the meridional non-uniformity of the eigensolution.

The zonal PV advection is further separated into two components, $\bar{u}_{2} v_{x x}$ and $-\bar{u}_{2} u_{y y}$. The former has a stabilizing effect, and the latter resembles the $\beta$ effect altering the local temporal growth rate and the phase speed of the perturbation. The positive zonal velocity produces similar wave behavior as a negative $\beta$, which is inferred by the effective $\beta$ term: $\beta-F \ell^{2}$. An increase of the intensity of the largescale on-shore flow is equivalent to a decrease in $\beta$, which leads to an increase in the phase speed. The destabilizing effect caused by this effective $\beta$ is overcome by the stabilizing effect of $\bar{u}_{2} v_{x x}$, so that overall the zonal advection of the perturbation PV by the large-scale eastward flow stabilizes the boundary current.

The influence of the meridional PV advection is accomplished mainly by the meridional velocity adjustment, accelerating or decelerating the perturbations. The group velocity is increased where the large-scale flow is southward, and reduced where the large-scale flow is northward. The altered group velocity and phase speed can affect the spatial growth rate $\ell_{i}$ through $\ell_{i} \simeq \omega_{i} / c_{g}$, and $l_{r}$ by $\ell_{r}=\omega / c_{r}$ which in turn changes $\omega_{i}$ and further $\ell_{i}$. This mechanism is confirmed by an examination of the relationship $\ell_{r} \simeq \omega_{i} / c_{g}$. Over the region where the jet is accelerated, the perturbation is elongated in both the meridional and the zonal direction, the spatial growth rate is reduced, and vice versa.

The WKB solutions are solved under the assumptions that the meridional scale of the perturbation is much smaller than the scale of the large-scale flow. The frequency is set to be constant, -0.2 , which corresponds to an unstable frequency as shown by results in Chapter 2. The streamwise variations shown here are small because of the weak large-scale flow. They are expected to become large once the strength of the large-scale flow is increased. We test and validate the WKB solutions by directly solving equation 4.26 using a numerical model in the next section. 


\subsection{Numerical simulations}

In this section, the relationship between the temporal and spatial instability, and results of the WKB solution are tested using linear numerical simulations, where the inviscid quasi-geostrophic vorticity equation 4.26 is solved directly by a numerical model. Unlike the WKB formulation where higher order terms are neglected, the equation solved by the numerical model retains all the linear terms. We retrieve the

parallel meridional jet stability problem by setting $\bar{U}_{2}=0$. Two cases are presented here. One case concerns the response of the system to a spatially localized forcing oscillating with a fixed frequency, the other is about the response of the system to a initial pulse perturbation. The numerical results are also compared with eigenvalue solutions. Both the partial-gyre and the double-gyre flow are studied.

\subsubsection{Spatial instability of a boundary jet with a localized forcing}

In a convectively unstable system, the local long-time response of a fixed station to an initial pulse perturbation decreases with time. If such a system is excited by a wave maker with a constant frequency, the response of the system corresponds to a spatially amplifying wave which oscillates with the frequency of the wave maker. We use this concept to test the relationship between the spatial and the temporal growth rate which is described by $\ell_{i}=\omega_{i} / c_{g}$ in Section 4.2 , also test the two influences of the partial-gyre flow by comparing the eigen-solutions and results of numerical integrations.

The relationship between the spatial and the temporal growth rate is tested in a case without the large-scale flow $\left(\bar{U}_{2}=0\right)$. The model is zonally bounded and meridionally periodic. The boundary jet is represented by a half Bickley profile flowing southward. The domain size is $W_{x}=30$ in the zonal direction and $W_{y}=150$ in the meridional direction, non-dimensionalized by the width of the boundary current. We forced the model by a wave maker with a frequency $\omega_{r}$ localized at an upstream 
fixed location, say at $y=y_{f}$. The wavemaker generates a spatially amplifying wave traveling southward. At the southern end of the model domain, an sponge layer is imposed to damp the signal, prevents the energy recycling from contaminating the spatial pattern of the amplifying mode. One snapshot of the spatially amplifying wave is shown in Figure 4-10. The integration is stopped at $t=250$ when the system is well developed. The spatial growth rate of the numerical simulation is calculated by a best fit of the signal to $A \exp \left(\ell_{i} y\right) \sin \left(\ell_{r} \mathrm{y}+C\right)$ where $A$ and $C$ are constants. The numerical results are also compared with eigenvalues.

Figure 4-11 shows that the spatial growth rates $\ell_{i}$ (lower panel) calculated directly from the eigenvalue problem of the spatial instability (blue line) and from the eigen-solutions $\left(\omega_{i}, c_{g}\right.$ in the upper panel) of the temporal instability. Two solutions (blue and red line in lower panel) agree well especially for small growth rates. The discrepancies between these two solutions increases for larger growth rates, although are still small, because their relationship is derived under the assumption of temporal growth rates being small (Gaster, 1962). Both the temporal (the blue line in the upper panel) and spatial growth rate (lower panel) have a maximum value around $\omega_{r}=0.38$.

In the numerical model, we conduct six simulations with six different frequencies, $\omega_{r}=0.1,0.2,0.3,0.4,0.5,0.6$. The spatial growth rates are shown in Figure 4-11 by the squares. They agree with the eigenvalues over the low frequency range, but not so well over the high frequency end. The reason for the large discrepancies over the high frequency end is unclear, but the model resolution is the primary suspect, as the errors of the fitting (shown by the error bars) also become large when the forcing frequency is high.

Next we add a large-scale flow to the problem, and compare again the WKB eigenvalue solutions with numerical results. Instead of the previous comparison, where the eigenvalues are based on the zeroth order equation 4.45, the numerical model uses the linear equation 4.32 . 


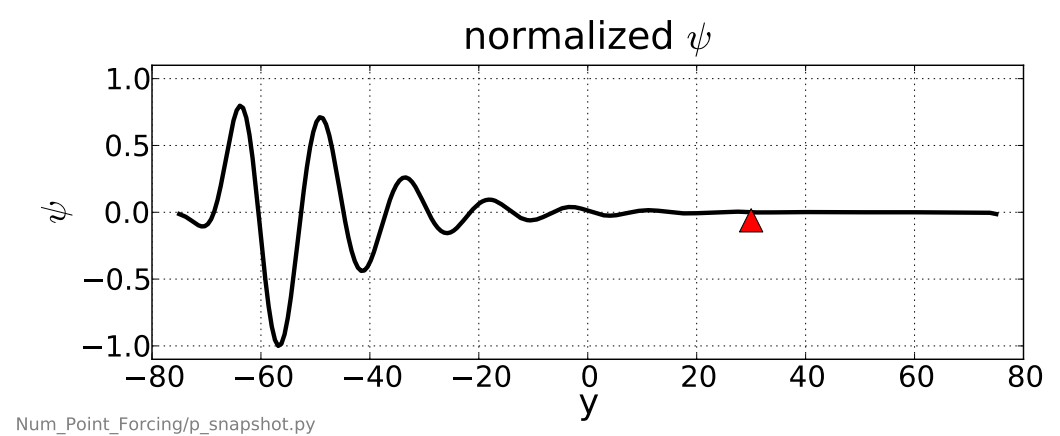

Figure 4-10: The spatial structure of a spatially amplifying wave generated by a wavemaker (indicated by the red triangle) with a frequency $\omega_{r}=0.4$ at $y=30$. The parameters are $\beta=0.01, \bar{U}_{2}=0$.

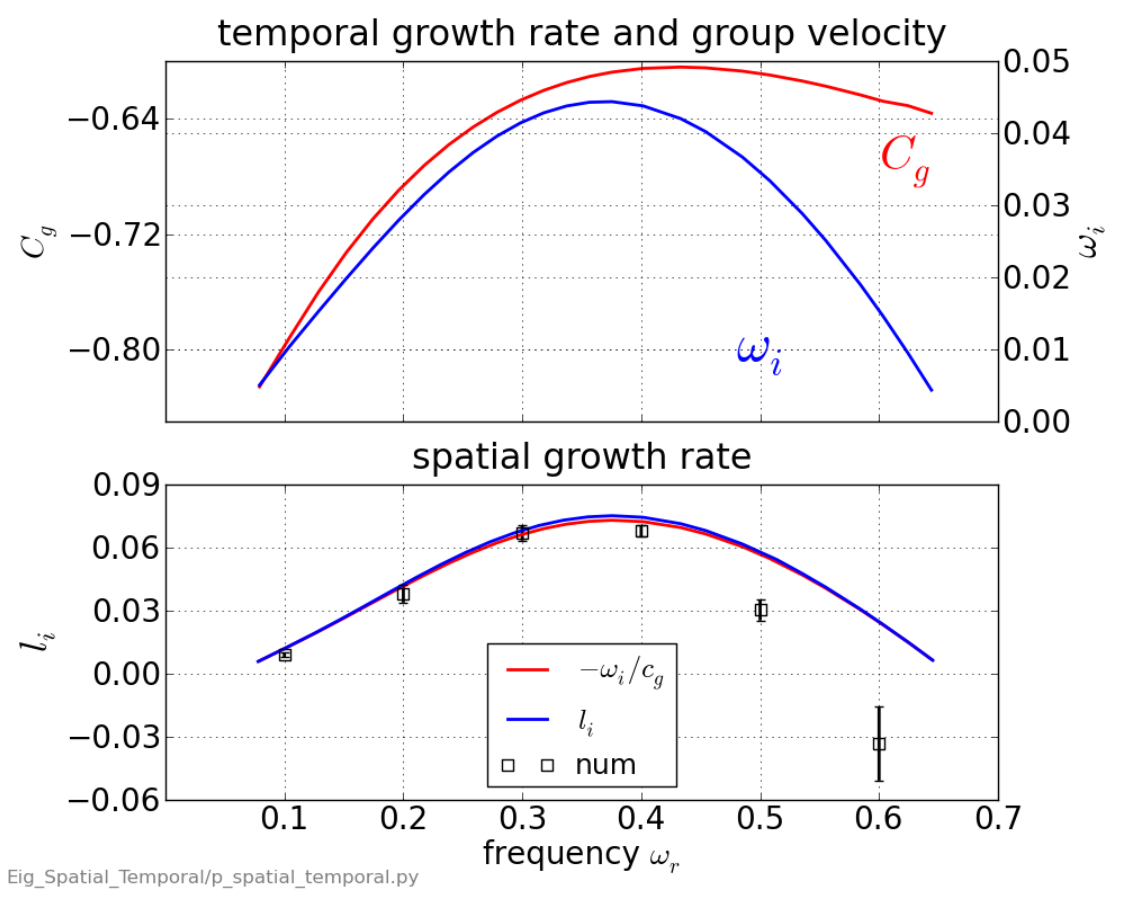

Figure 4-11: The relationship between the temporal and the spatial growth rates. The current is represented by a half Bickley profile along the eastern boundary. The spatial growth rate $\ell_{i}$ (blue line in the lower panel) is calculated directly from the eigenvalue problem for the spatial instability given $\omega_{r}$, which together with $\omega_{i}$ (blue line in the upper panel) are the eigenvalue for the temporal instability. $c_{g}$ is calculated by $\left[\omega_{r}\left(\ell_{r}+\delta\right)-\omega_{r}\left(\ell_{r}-\delta\right)\right] / 2 \delta$ where $\delta=10^{-4}$ and shown by the red line in the upper panel. $\omega_{i} / c_{g}$ is the derived spatial growth rate from the temporal instability problem and is shown by the red line in the lower panel. The squares indicate the spatial growth rates computed from numerical simulations forced by an oscillating source. The error bars are calculated from the standard errors in the least-square fitting. 
Figure 4-12 shows the spatial growth rate at $y=0$ for the problem with the partial-gyre flow. The changes are due to the influence of the zonal PV advection - The growth rates calculated by two methods agree with each other qualitatively. The same discrepancies between the eigenvalues and the numerical simulations over the high frequency range (Figure 4-11) still exist in the background-flow-influenced problem. The case with no large-scale flow is plotted in red in the figure as a reference. By comparing the black and red dashed lines, we observe that the background flow stabilizes the jet for all frequencies including $\omega_{r}=-0.2$, which is extensively discussed in the WKB solution in the previous section. The numerical simulations qualitatively confirm the stabilizing effect of the large-scale flow (the circles with errorbars, compare the red with the black), with the same large discrepancies over the high frequency end.

The effect of the meridional velocity adjustment is shown by the streamwise structure of the spatial growth rates (Figure 4-13). The numerical results agree with the eigenvalues with some discrepancies (differences between the solid and dashed lines), which are small at $y=0$ but becomes relatively large for large $|y|$. The differences between the red lines and the black lines at $y=0$ are caused by the influence of the zonal PV advection, which is the stabilizing effect. The slope shows the velocity adjustment effect, which is caused by the meridional PV advection, and becomes steeper when $\bar{U}_{2}$ becomes larger.

In summary, the numerical simulations of the spatially amplifying waves generated by a localized forcing agree with the eigenvalue solutions although there are some small discrepancies. The simulations with high frequency show large discrepancies compared with the eigenvalue solutions. The reason for this large discrepancies is unclear. The insufficient model resolution might be the cause. One merit of this study is that we can control the forcing frequency, which is not feasible for the pulse instability discussed in the following section. 


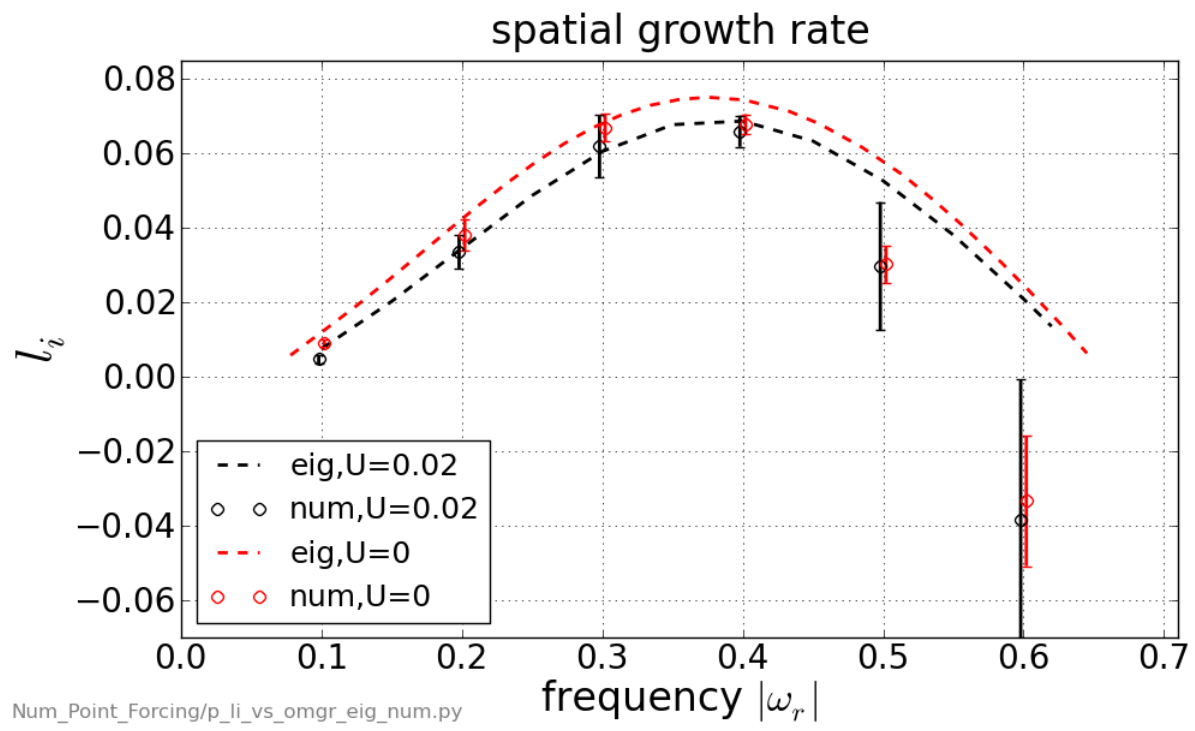

Figure 4-12: Spatial growth rates at $y=0$ calculated from numerical simulations (circles) and from eigenvalue solutions (lines) with $\beta=0.01$. The red color represents the parallel case with $\bar{U}_{2}=0.0$, and the black color represents the case with $\bar{U}_{2}=0.02$. The values for the numerical simulation are calculated base on the growing pattern over $-10<y<10$.

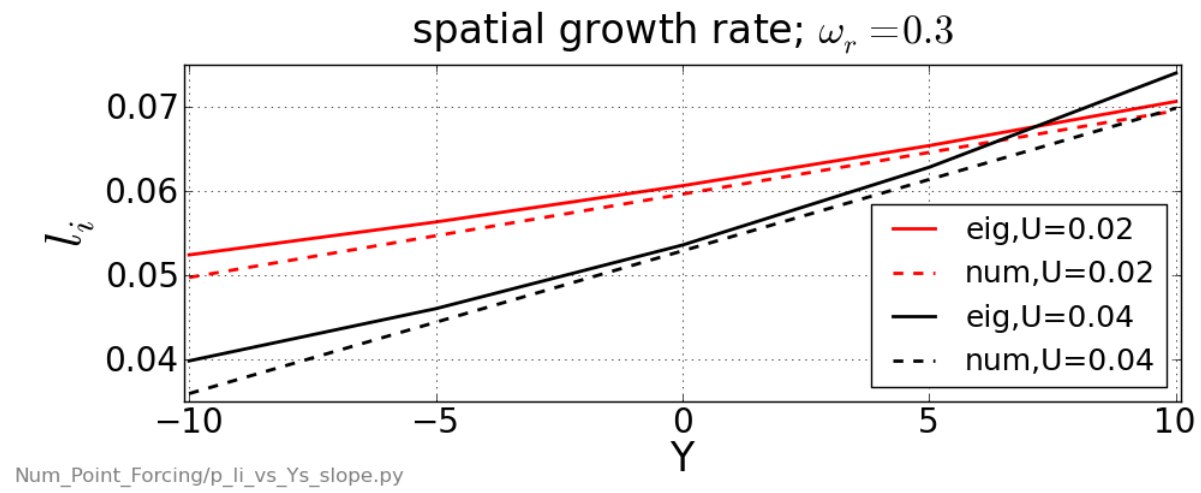

Figure 4-13: Spatial growth rates with different strengths of the background flow. 


\subsubsection{Pulse instability of a parallel boundary jet}

In this section, numerical simulations are used to show how a pulse initially evolves in a parallel boundary jet. Such a pulse can be generated in the real ocean by a sudden wind onset or a transient hurricane. The numerical model is the same one used in the previous section. In the following two sections, we will add the partial and double-gyre into the model to study the influence of large-scale flows.

The model is initialized with the pulse perturbation,

$$
q=\exp \left(\frac{-\left(x-x_{0}\right)^{2}-\left(y-y_{0}\right)^{2}}{W^{2}}\right)
$$

and integrated forward in time. The pulse perturbation becomes unstable and grows downstream as shown in Figure 4-14. The initial Gaussian perturbation contains a very wide range of wavenumbers. The wave packet that emerges after a long time corresponds to the most unstable mode. The wave ray is not clear at the beginning because the perturbation is in its adjustment phase. It becomes evident when $t$ becomes large.

This boundary jet is convectively unstable and the signal is growing and traveling downstream. The local response at a fixed location is transitive. The perturbation grows before decaying to zero (the right panel of Figure 4-14) as a growing wave packet passes the observing station which is marked by the dashed line in the middle panel. The phase delay of blue lines comparing with red lines shown in the middle panel means the phase speed becomes smaller when $\beta$ increases, which confirms the eigensolution that $\beta$ decreases the phase speed. Another phenomenon found in the eigen-solution is that an increase of $\beta$ leads to a decrease in the growth rate. This decrease is also observed in this simulation as is shown in the left panel of Figure 4-14 where the logarithms of the peak of the envelops are plotted. The growth rate for $\beta=0.05$ is smaller than that for $\beta=0$. The right panel shows a local response to a wave packet passing the station $y_{s}$. The red line arrives earlier and has larger 


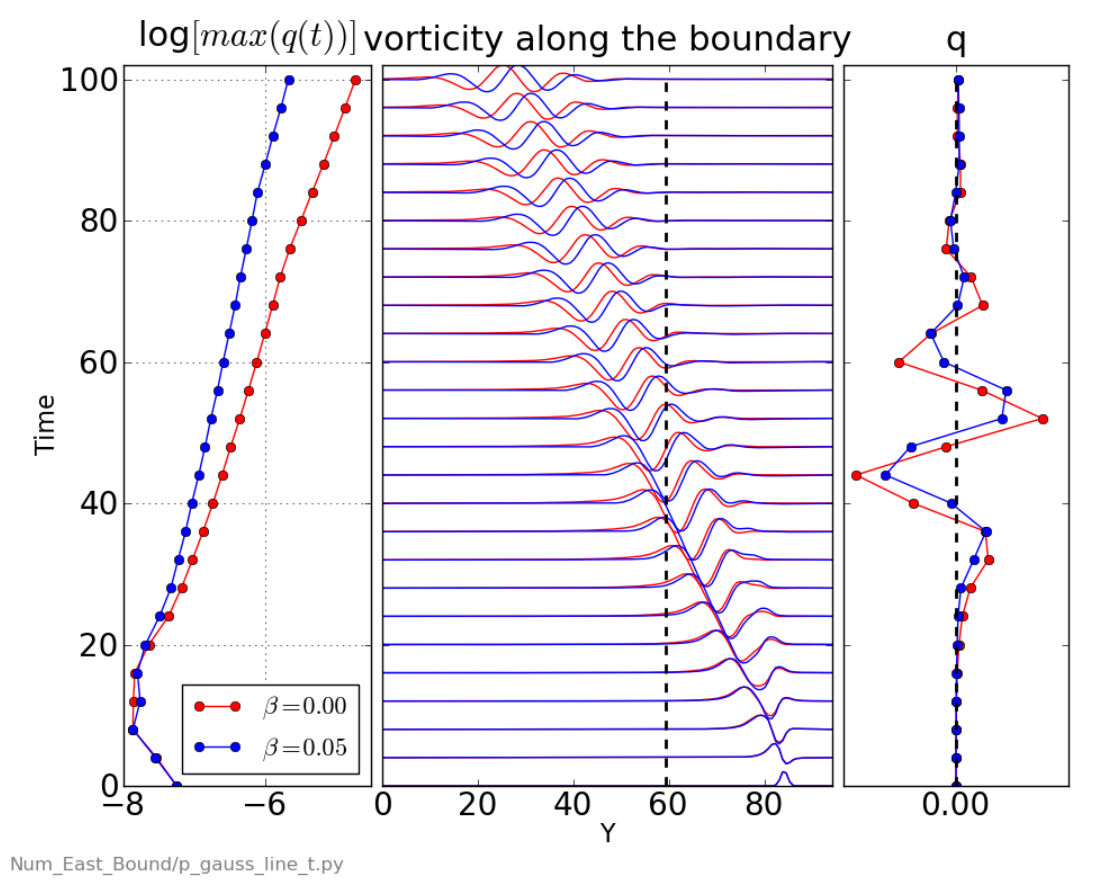

Figure 4-14: The pulse response of a parallel boundary jet. The pulse has a Gaussian initial condition, and evolves to a wave packet for large t. In the middle panel, each line is a snapshot of potential vorticity (normalized by the maximum value) along the boundary at $x=x_{0}$ which is arbitrary as long as $x_{0}$ is inside of the jet. Red lines represent the $\beta=0$ case, and blues lines $\beta=0.05$ case. The phase delay of blue lines relative to red lines indicates the signal is retarded by the $\beta$ effect. The $\log [\max (\mathrm{q})]$ is shown in the left panel. The smaller $\mathrm{q}$ for $\beta=0$ than for $\beta=0.05$ means including $\beta$ decreases the growth rate of the perturbation. The right panel shows the local response of $q\left(x_{0}, y_{s}, t\right)$ as a function of time at $y_{s}$ indicated by the dashed line in the middle panel.

fluctuation amplitude, which qualitatively confirms our conclusion about the two $\beta$ effects, that reduce the phase speed and growth rates, from a different perspective.

To conclude, the half Bickley jet along the eastern boundary is convectively unstable. The eigensolutions are qualitatively confirmed by a numerical study. For the eastern boundary jet, the increase of $\beta$ reduces the phase speed and the growth rate of the perturbation. In the following two sections, we quantify the comparison and also investigate the influence of the large-scale flow. 


\subsubsection{Pulse instability of a boundary jet modified by a partial- gyre flow}

We study the influence of the large-scale flow on the boundary jet stability by adding $\bar{\psi}_{2}=-F(x) y$ to the previous simulation. $F(x)$ is the same as what is used in WKB analysis. The large-scale flow has a uniform onshore flow in the interior and diffluent structure in the boundary region. The two dimensional example of $\bar{\psi}_{2}$ is shown in Figure 4-4.

The numerical model solves the linearized barotropic QG equation. The model is zonally bounded and meridionally periodic. The east and west walls have the slip boundary condition. The boundary jet is represented by a half Bickley profile flowing southward. The domain size is $W_{x}=30$ in the zonal and $W_{y}=70 \pi$ in the meridional direction in a non-dimensional form scaled by the width of the boundary current. The initial perturbation is a localized Gaussian patch located inside of the jet near the northern domain boundary. The model is run without the large-scale flow from $t=0$ to $t=100$, so that the initial Gaussian perturbation can develop to form a wave packet supporting the maximum growth. The solution at $t=100$ is used to initialize two following simulations. The first simulation is a continuation of the no-large-scale flow simulation from $t=100$ to $t=240$. The second simulation is the same as the first simulation but with the large-scale flow $\left(\bar{U}_{2}=0.02\right)$. It is also run from $t=100$ to $t=240$. The comparison of those two simulations implies the influence of the large-scale flow on the stability of the boundary jet.

Figure 4-15 shows the qualitative differences in the peak amplitude (left), the meridional wave structure (middle) and the response at a fixed station (right) between the two simulations. Three phenomena are observed. First, given the same sufficiently long time (indicated by the $y$-axis in the figure) to develop, the peak of the perturbation grows slower in the jet with the background flow (JWBG) than in the jet without the background flow (JOBG) (left panel). Second, at a fixed station upstream $(y>0)$, the signal of the perturbation is larger and vanishes slower in JWBG 


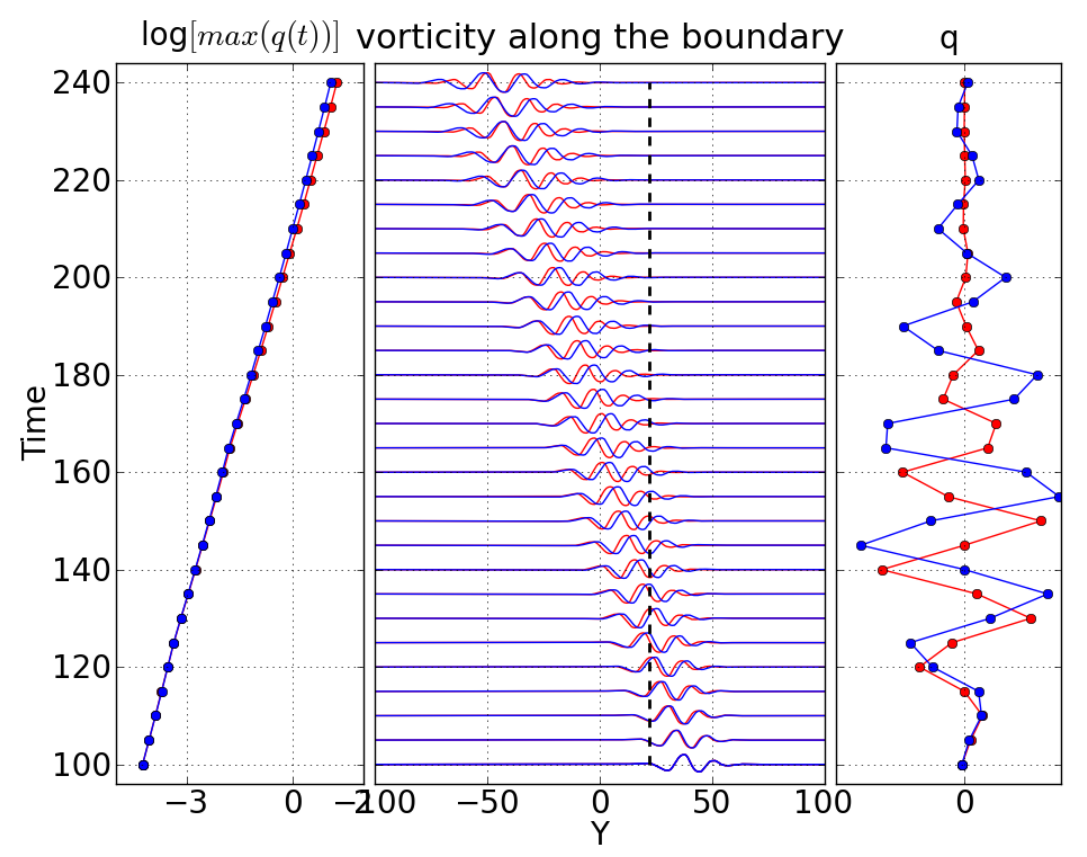

Figure 4-15: The same plot as Fig 4-14 but for the comparison of simulations with different large-scale flows. Red lines show the first simulation (no large-scale flow), and blue lines show the second simulation (with large-scale flow). The two simulations start from the same initial condition.

than in JOBG (right panel). Third, in the middle panel, the travel of the initial wave packet is retarded in JWBG upstream $(y>0)$ (blue lines fall behind red lines), and accelerated when it passes $y=0$ (blue lines catches up red lines). It qualitatively confirms previous WKB solutions. However, the differences are small because of the weak large-scale flow. In order to quantify the differences, we use several techniques to calculate the signal properties: the wave packet, the group velocity, the phase speed, and the growth rate.

Figure 4-16 shows the procedure for calculating the wave properties with a focus on the meridional structure. A 3rd-order spline fitting is used to form the wave packet from the streamfunction. The spatial growth rate $\ell_{i}$ is defined as the spatial derivative of the logarithm of the peak of a wave packet, and the group velocity is calculated by taking the time derivative of the $y$-location associated with that peak. The phase speed is computed by the same procedure but by tracking the location of a 
fixed phase instead of tracking the peak of a wave packet. Notice that the derivatives can enlarge the noise, so that we use the least square technique, when it is needed, to filter out the noise. The detailed procedure is discussed in the caption of Figure $4-16$.

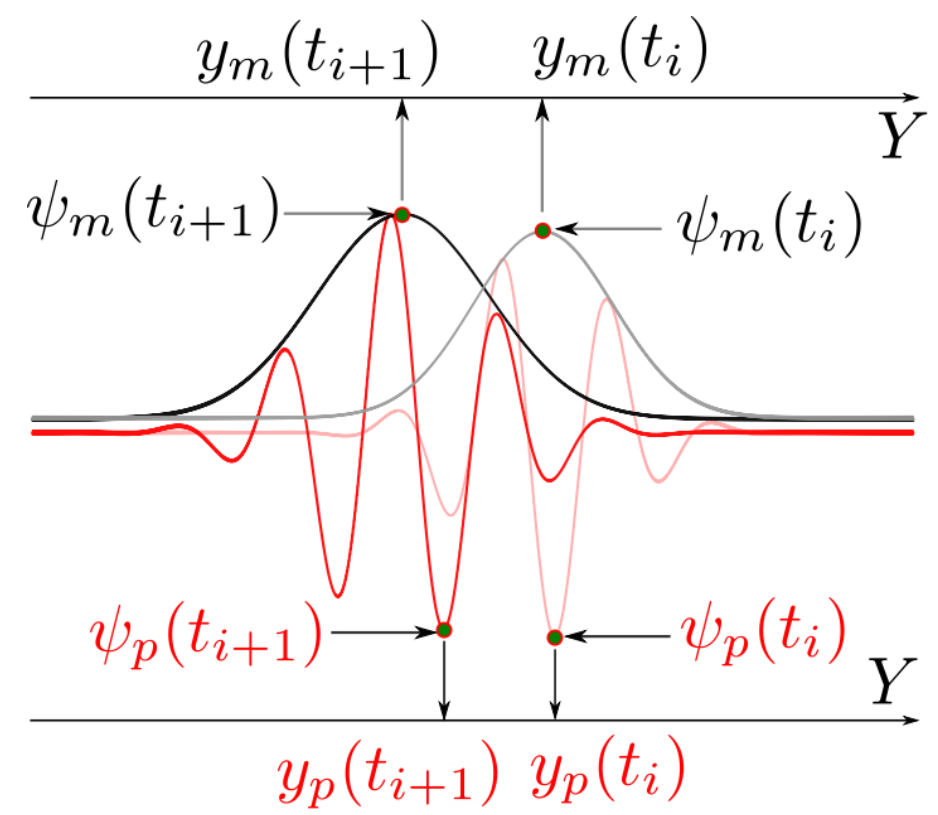

Figure 4-16: This sketch shows the procedure following which the growth rate, the group velocity and the phase speed are calculated. Red lines shows the alongboundary structure of the streamfunction. Black lines represent wave packet calculated from the cubic spline (Dierckx, 1995) fitting of the streamfunction. $t_{i}$ marks the time at step $i$ of the integration. $\psi_{m}$ refers to the peak of the wave envelop, and $\psi_{p}$ refers to the local maximum. Each peak (both local and of the envelope) has three properties, the amplitude $\psi$, the location $y$, and the time $t$. By linking those properties, we can formulate $\psi_{m, p}=f_{m, p}(t)$ and $y_{m, p}=g_{m, p}(t)$. The spatial growth rate is defined as $\ell_{i}(t)=\frac{d \log \left(f_{m}\right)}{d t} \frac{d t}{d y_{m}}$, the group velocity is $c_{g}(t)=\frac{d y_{m}}{d t}$ and the phase speed is $c_{p}(t)=\frac{d y_{p}}{d t}$. We are interested in those properties corresponding to spatial locations, so we can interchange $t$ and $y$ by $t=g^{-1}(y)$.

In the following discussion, we explore the numerical results focusing on the two influences proposed by the WKB solutions. First we discuss the influence of the meridional PV advection, i.e., the effect of the meridional velocity adjustment, followed by the discussion of the influence of the zonal advection. The conclusion is that the influence of the large-scale flow on the pulse instability follows the mechanisms 
suggested by the WKB solutions.

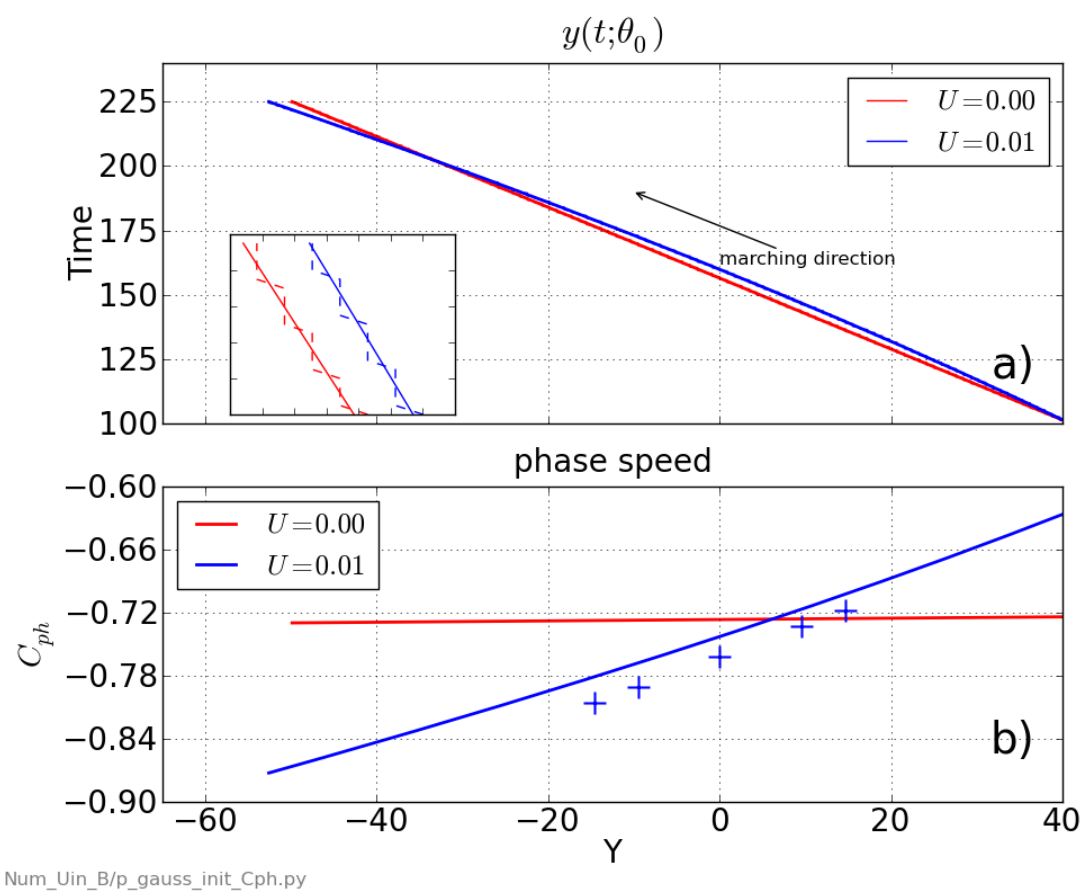

Figure 4-17: The calculated phase speed for two cases. Red lines show the results for the JOBG. Blue lines show the results for JWBG, and the zonal velocity for the background flow in the interior is $\mathrm{U}=0.01$. (a) shows the $t=g^{-1}(y)$ of a fixed phase. The ordinate represents the time, the abscissa represents the y-location of the marked phase at the corresponding t. The signal travels southward indicated by the arrow. The noise is too small to be detected in the panorama, but can be clearly seen in the zoomed-out subplot of (a) where the dashed line is from data and the solid line is the least square solution. The noise could be amplified once y derivative is taken to calculate the phase speed. We use the least square technique to fit the solution to a second order polynomial err $=y_{p}(t)-\left(a t^{2}+b t+c\right)$. The phase speed is $c_{p}=2 a t+b$. Change t to y gives us $c_{p}=2 a g^{-1}(y)+b$ and shown in panel (b).

Figure 4-17 shows the calculated phase speed. The signal in the parallel boundary jet travels almost in a constant phase speed (red line in b)). The phase speed of the wave packet develops a clear spatial structure when the background flow is included. The signal travels faster in the downstream direction. As the original 2nd-order polynomial fitting of $y_{p}$ convincingly represents the behavior of the signal, we believe the influence of the partial-gyre large-scale flow on the phase speed is mainly linear as shown by panel (b). It is physically logical as the background flow velocity has 
linear structure in $y, V=-F\left(x_{0}\right) y$.

The phase speed at $y=0$ is larger for $\bar{U}_{2}=0.01$ (blue line panel b) than for $\bar{U}_{2}=0$ (red line panel b). This phenomenon is also shown in WKB solutions. The proposed mechanism is that part of the zonal transport of perturbation PV by the large-scale flow acts like $-\beta$ and changes the phase speed through the $\beta$ effect which is discussed in the previous chapter.

The eigensolutions (blue crosses) are calculated by the following procedure. First we fit the time series of a numerical simulation at the corresponding $y$ to a sinusoidal signal to get the frequency $\omega_{r}$. Given the frequency, we solve the eigenvalue problem for the spatial instability to get the complex wavenumber $\ell=\ell_{r}+\mathrm{i} \ell_{i}$. The phase speed is then computed according to $c_{r}=\omega_{r} / \ell_{r}$. The spatial growth rate $\ell_{i}$ will be discussed later.

The phase speed from the numerical simulations and from the eigenvalues do not match perfectly, but the discrepancy is less than $5 \%$ and the slope agrees very well (the blue line and blue crosses in panel b)). Those small differences come from two possible sources. One is that errors may be introduced by the fitting procedure for the frequency because a wave packet contains a range of frequencies instead of only one frequency. Another possible reason is that the higher order terms which are neglected in the WKB analysis may play roles in the numerical simulations. In this example, whichever introduces the differences, its influence is small and secondary. Later, we further study those discrepancies for different large-scale flow intensities by varying $\bar{U}_{2}$ and find that it is highly possible those discrepancies are caused by those neglected higher-order terms in the WKB analysis.

The influence of the meridional PV advection, modifies the phase speed, and also the group velocity. The group velocity is calculated as the speed of the peak of a wave packet. One example of the spline-fitted wave packet is shown in Figure 4-18. From the Hovmöller diagram (c), we can see the wave packet is clearly elongated and accelerated as the signal travels downstream in the case with a large-scale flow. 


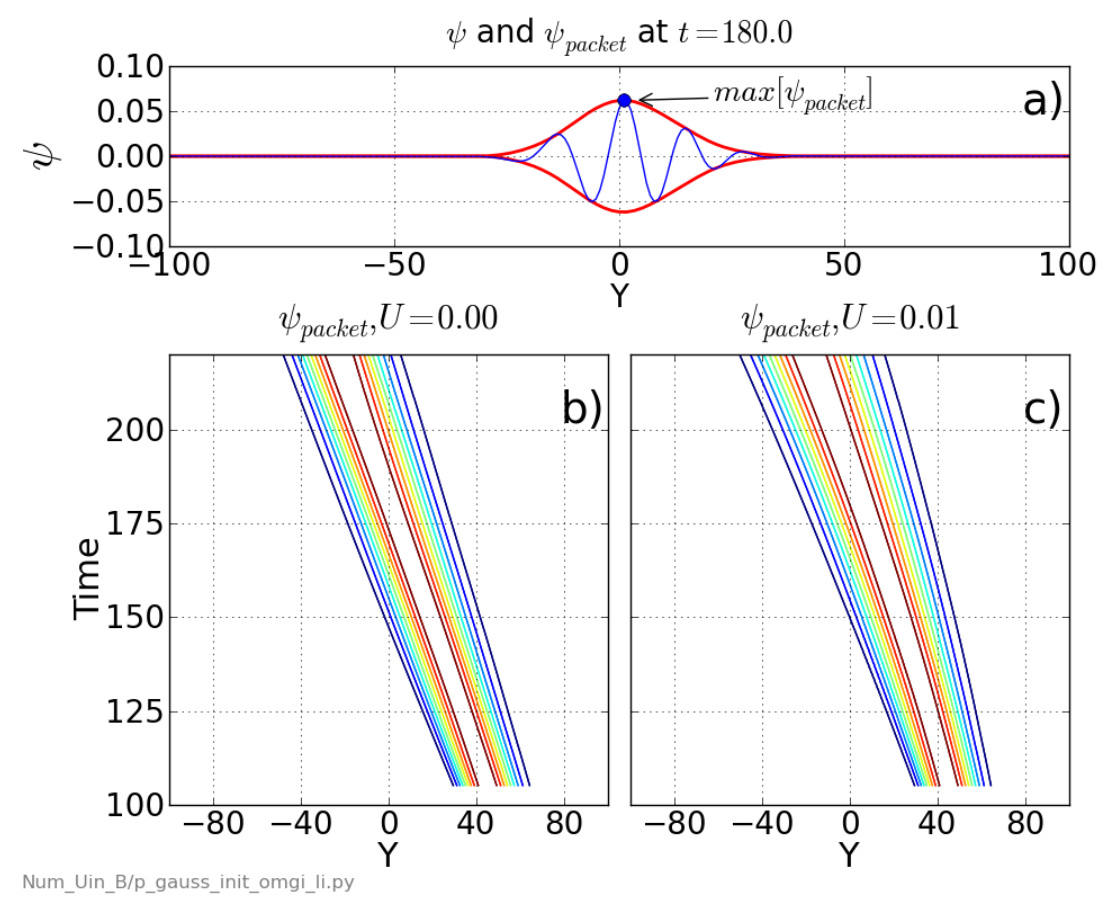

Figure 4-18: (a) shows the snapshot of the streamfunction as a function of $y$ at a fixed $\mathrm{x}$ where the maximum of the streamfunction resides. Red lines show the 3rd-order spline fitting. (b) is the hovmoller diagram of the wave packet shown by the red curve with positive values for the case without the background flow. (c) is the same as (b) but for the case with the partial-gyre large flow. Both cases start from the same initial condition, but the large-scale flow changes the wave packet structure as the signal travels downstream.

The calculated group velocity is shown in Figure 4-19. The same phenomenon observed in the phase speed also appears here, except that the group velocities with/without the large-scale flow are the same at $y=0$ for the two cases. This is a very interesting feature. It implies that the $\beta$ effect changes the phase speed of the unstable modes, but without distinguishing different modes (no wavenumber dependence). The $\beta$ effect does not change the group velocity, and the only factor that changes the group velocity is the meridional velocity adjustment. However, we have not found a reasonable explanation. It is also possible that group velocity varies, but the changes are too small to be detectable. The mechanism remains an open question. 


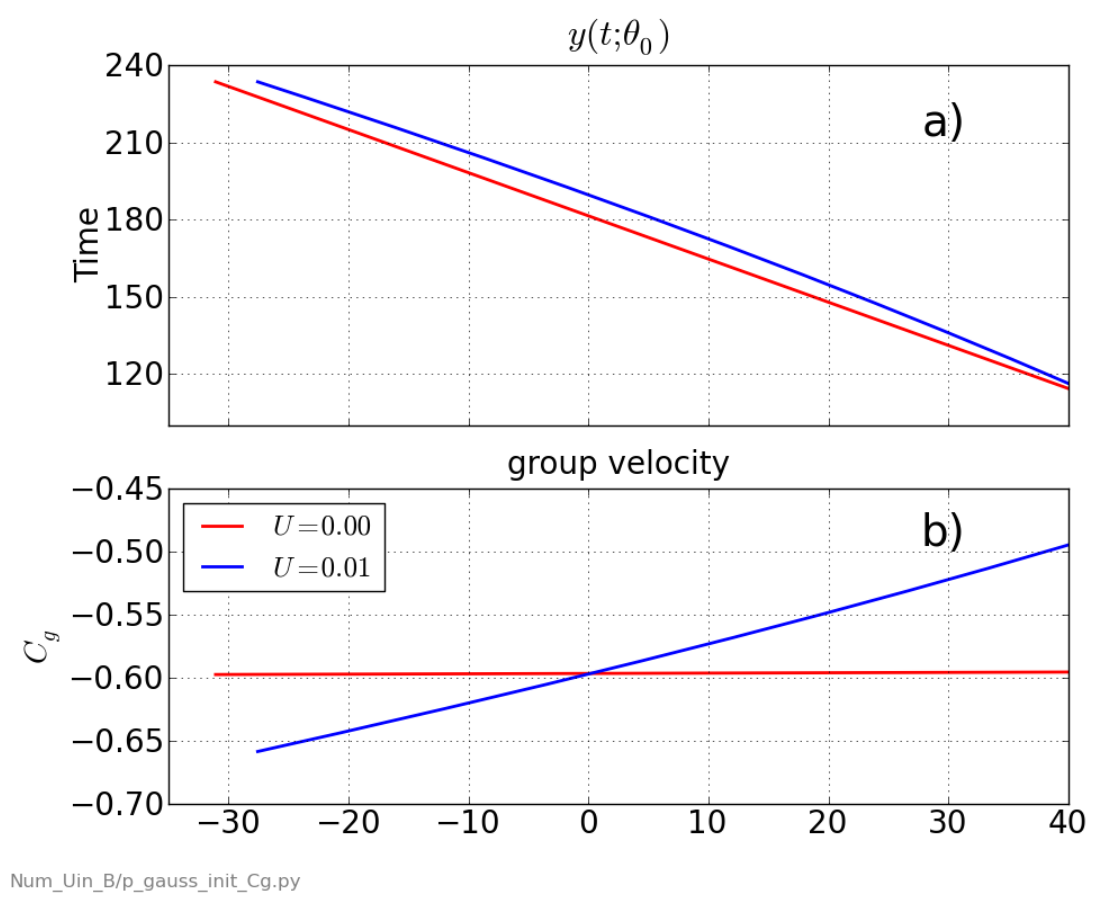

Figure 4-19: The same as Figure 4-17 but for the group velocity. The same group velocity in both cases at $y=0$ means that the eastward flow does not change the group velocity.

Next we examine the spatial growth rate $\ell_{i}$ as is shown in Figure 4-20. The spatial growth rates of the numerical simulations are the spatial derivative of the amplitude of the peak of the wave envelope. The eigenvalues are computed from the spatial instability given a real frequency which is the best fit of the time series at specific $y$ from the numerical simulations. In the JOBG case, the spatial growth rate for the wave envelope is constant (red line in panel b). Once the partial-gyre flow is added, the spatial growth rate changes and shows a meridional non-uniformity, and the slope shows the effect of the meridional velocity adjustment (blue line in panel b). The stabilizing effect of the zonal onshore flow is shown by the discrepancy at $y=0$ between the two cases (the difference between the red line and the blue line). The eigenvalues show similar slopes but with discrepancies. The result of the numerical simulation and the eigenvalues do not match perfectly at $y=0$. Those differences may caused by the higher order terms which are neglected in the WKB analysis, or 


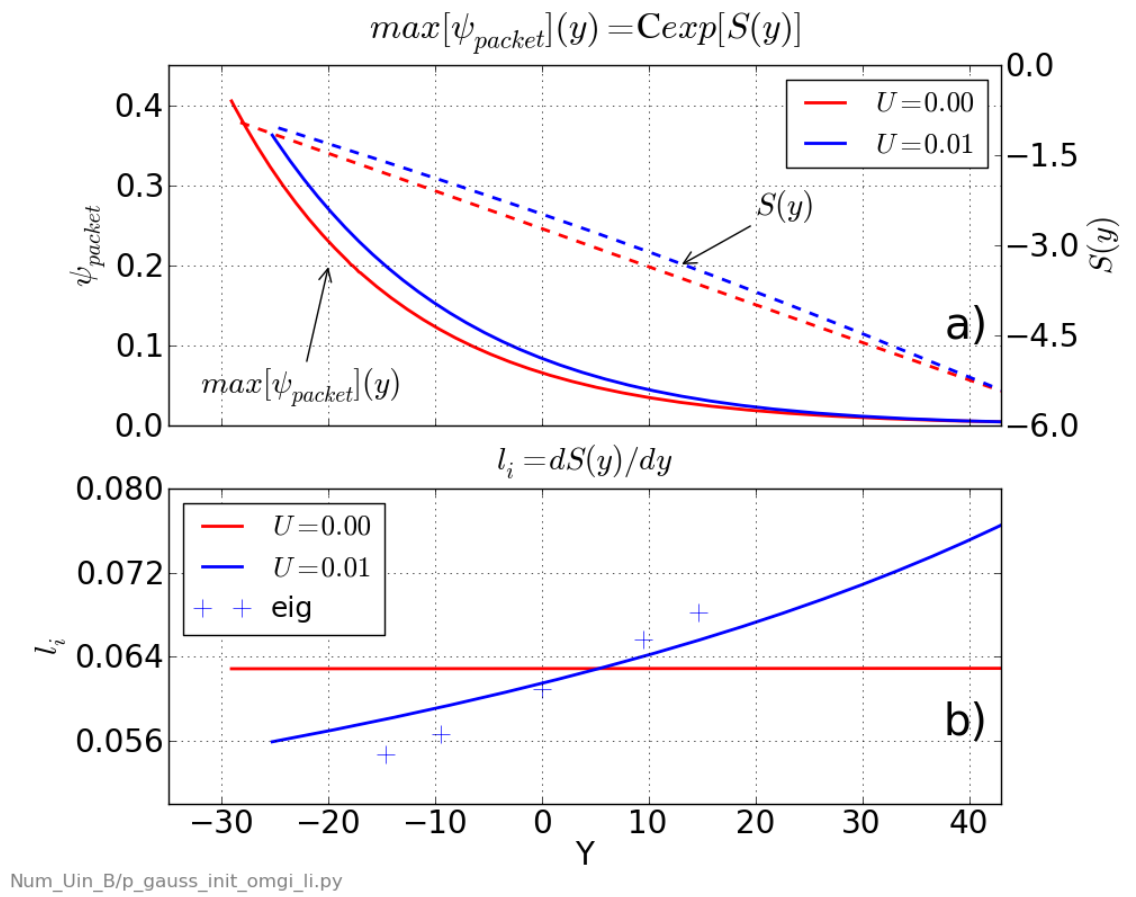

Figure 4-20: The spatial growth rate $\ell_{i}$. Red lines are for the case with a parallel jet. Blue lines are for the case with a partial-gyre. In panel (a), the solid lines show the actual amplitude of the peak of the wave packet, and the dashed lines are the logarithm of the amplitude, which should be a straight line if the wave packet grows at a constant rate. The spatial growth rate calculated from (a) is shown in panel (b). Detailed calculation procedure can be found in the caption of Figure 4-16.

the uncertainties introduced in the least-square-fitting calculations.

Figure 4-21 shows that the differences between the results of the numerical simulations and the results of the eigenvalues for the spatial instability at $y=0$. The differences increase following the increase of the intensity of the large-scale flow, which implies that they are caused by the neglected terms in the WKB analysis, because those neglected terms become large if the large-scale flow becomes stronger.

Finally, we present the behavior of a pulse perturbation in the boundary jet for different values of $\beta$, as shown in Figure 4-22. Including $\beta$ systematically decreases the spatial growth rate for all upstream and downstream locations. We know from the eigen-solutions discussed in the previous section that including $\beta$ decreases the growth rates of the unstable modes of the eastern boundary jet. The distance between 


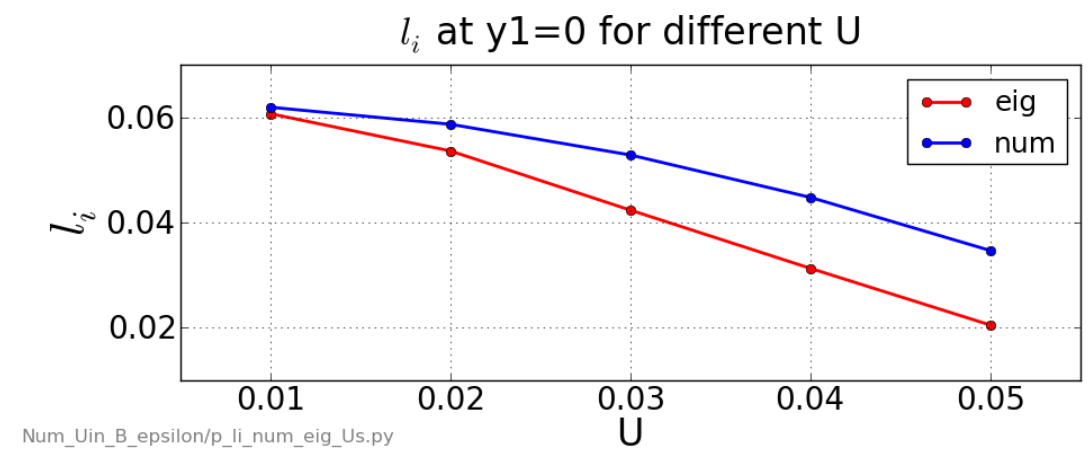

Figure 4-21: The spatial growth rate from the numerical simulations (blue) and eigenvalue solutions (red). Their discrepancies increase when the intensity of the large-scale flow becomes large ( $U$ increases).

the red line and the blue line is relatively uniform, indicating that the change in $\ell_{i}$ from $\beta=0$ case to $\beta=0.05$ case is almost constant. This is because $\beta$ does not change the group velocity and $\ell_{i}=\omega_{i} / c_{g}$. The result agrees with the eigensolutions.

To summarize, we investigated the pulse instability of the eastern boundary jet and the influence of the partial-gyre flow on the pulse instability using a time-dependent numerical model, and found that the results agree with the WKB eigensolution. A wave packet is able to grow to the same amplitude in a shorter distance (corresponding to the large $\ell_{i}$ ) over the upstream region, where the jet is slowed down by the largescale flow, than over the downstream region, where the jet is accelerated by the large-scale flow. The wave is stretched flowing downstream due to the effect of the meridional velocity adjustment.

For the experiments with the particular parameters used here, the agreement of the numerical simulation with the eigensolution discussed in previous section validates both the eigensolution and the numerical simulations. The discrepancies between the numerical results and the eigenvalues are due to higher order terms which are neglected in the WKB analysis. The method used to calculate the wave properties from the result of the numerical simulation provides us a useful tool to investigate the influence of more complex large-scale flows, for example the double-gyre flow, which is discussed in the following sections. 


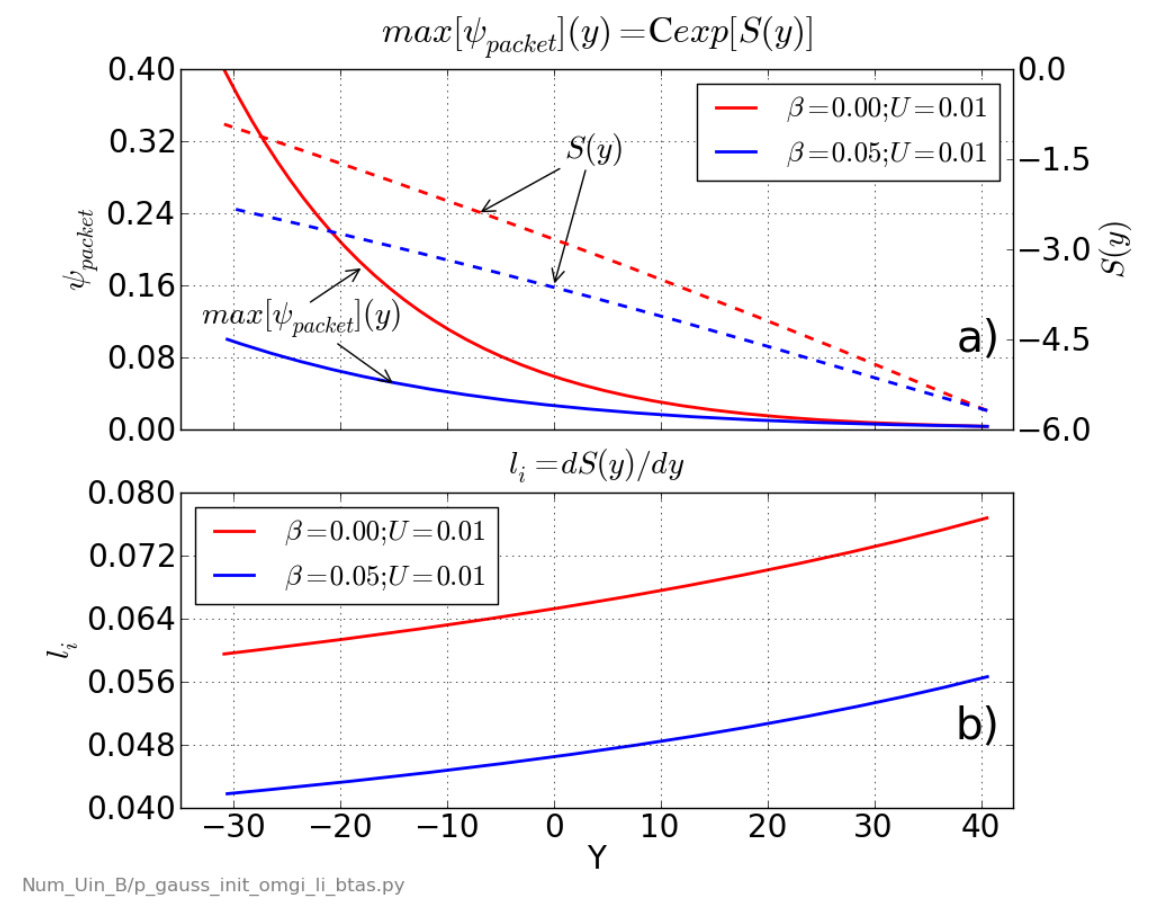

Figure 4-22: The $\beta$ influence. This figure is the same as Figure 4-20 but for different $\beta$. Red lines are for $\beta=0$ and blue lines are for $\beta=0.05$.

\subsubsection{Pulse instability of a boundary jet modified by a double- gyre flow}

The partial-gyre flow structure has such a simple structure that the WKB analysis and the interpretation of the results are made easier. However, this partial-gyre flow does not support a north-south periodic boundary condition, which makes the longtime integration less meaningful. We here extend our problem one step further by using the double-gyre flow to study the influence of a relatively more complex large-scale flow structure on the boundary jet instability. The double-gyre structure allows us to use periodic boundary conditions in the meridional direction. After integrating for a sufficiently long time, global modes exist for certain parameter ranges. In the following discussion, we use the linearized numerical model to investigate the problem of how the instability of a boundary jet behaves under the influence of a double-gyre flow. The model boundary conditions are the same as used in previous sections: the slip condition for the eastern and western walls and periodic condition 
in the meridional direction. The no-slip boundary condition can make the boundary current more unstable. However, we will focus on the slip boundary condition, and leave the effect of boundary conditions on the results shown in this section to a future study.

The discussion is organized in the following sequence. We first introduce the model setup, and its scale/strength relative to the boundary jet, carry out several simulations to study the pulse instability of the boundary jet under the influence of gyres, then investigate the beta effect on the instability of a boundary jet with gyres, and finally use random noise as a initial condition and study the relationship between the global mode and the intensity of the large-scale flow. We focus on the new features brought by the gyre flow rather than repeating the discussion similar to those brought by the partial-gyre flow.

\section{Model setup}

Figure 4-23 shows the double-gyre large-scale flow. The contours represent the streamfunction, and the black arrows represent the boundary jet structure. The zonal velocity is maximum in the interior but decays to zero at the side boundary, and the meridional velocity reaches a maximum along the boundary where the jet resides. The maximum meridional velocity $\bar{v}_{2 \max }$ is used as an index representing the relative strength of the large-scale flow because it is an important feature and can be easily compared with the velocity of the jet and the group velocity of wave packets.

The domain size is 30 in the zonal direction and $70 \pi$ in the meridional direction, non-dimensionalized by the width of the boundary current. This domain's meridional extent is much larger than its zonal extent. The large meridional extent provides enough distance for perturbations to evolve slowly. The small zonal extent is chosen because we try to resolve well the zonal structure of the boundary current but also to reduce the zonal grid number to save computating time. The zonal size of the domain is less relevant as long as the size is not too small (suppressing the instability 


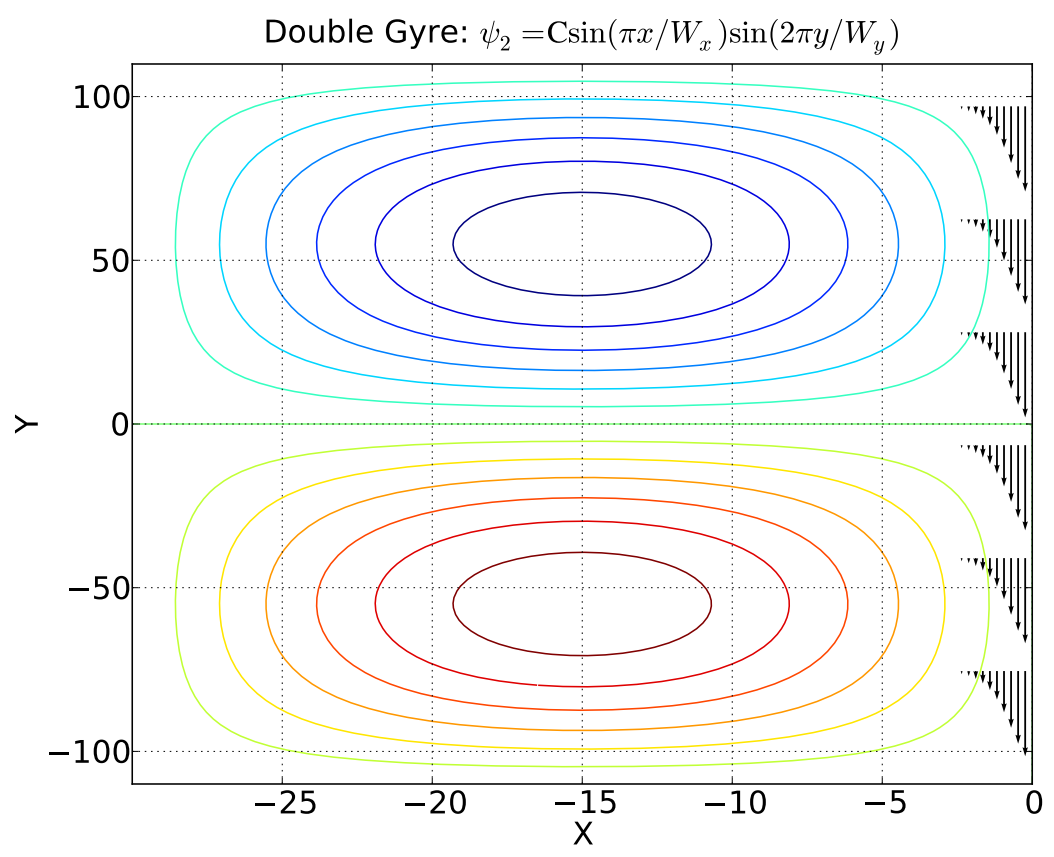

Figure 4-23: The streamfunction of the gyre large-scale flow, $\bar{\psi}_{2}=$ $\sin \left(\pi x / W_{x}\right) \sin \left(2 \pi y / W_{y}\right)$. The northern/southern gyre is cyclonic/anticyclonic. The profile of the boundary jet velocity is shown by black arrows. The relative scale between gyres and the boundary jet is qualitatively clear from the figure. The boundary jet is decelerated in the upstream, but accelerated in the downstream.

as discussed in the previous chapter) and the value of $\beta$ is not too large (supporting significant zonal propagations). A sponge layer is applied along the western boundary to prevent wave reflection.

Figure 4-24 shows the vorticity structures of the mean state (including the largescale flow and the jet) for large-scale flows with different amplitudes. The main feature of the vorticity field comes from the jet even when the large-scale flow is relatively strong. The vorticity contribution of the large-scale flow is small adjacent to the boundary.

Figure 4-25 and 4-26 show how the developed initial pulses evolve in two cases with different flow intensities. When the large-scale flow is weak, the jet is still convectively unstable as the wave packet is growing and also advected away from a fixed location (Figure 4-25). The large-scale flow slows down the wave propagation and the local 

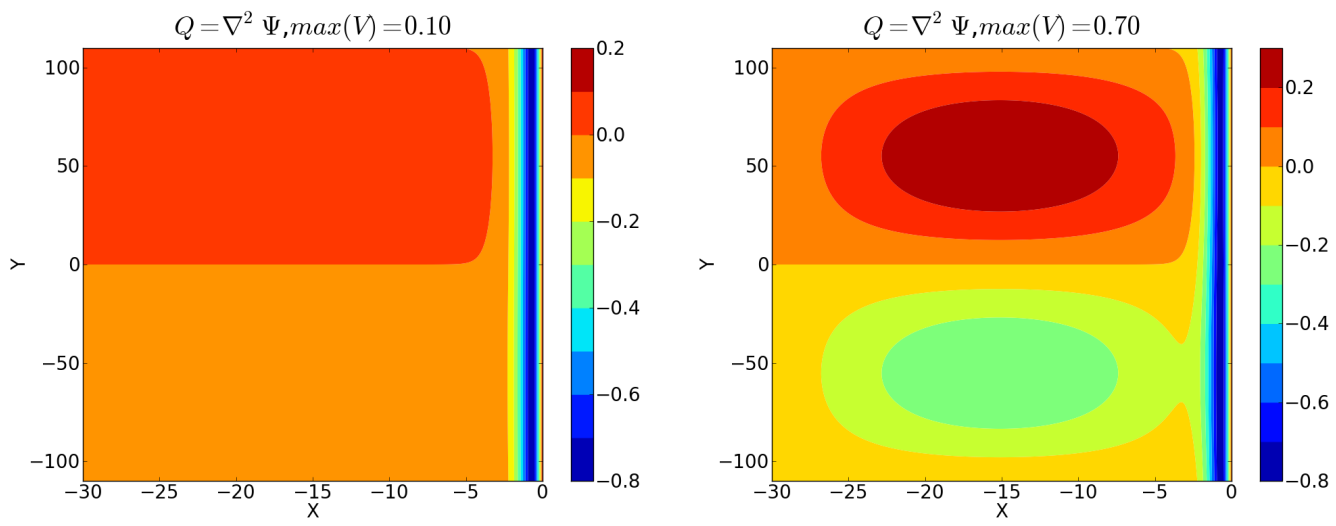

Figure 4-24: The total vorticity of the mean state including the large-scale flow and the boundary jet. When the large-scale flow is weak, the vorticity structure of the jet is barely modified (left). When the large-scale flow is strong, the vorticity field along the boundary still preserves the vorticity structure the boundary current.

decay rate over the upstream region (blue line in the right panel of Figure 4-25), which is also found in the case with the partial-gyre flow. Once the large-scale flow intensity is strong enough, some upstream locations show growing signals and yield a switch of the instability from convective to absolute (Figure 4-26). The wave packet is significantly stretched downstream. Consequently, the growth of the wave packet becomes smaller because of the horizontal spreading (left panel). Figure 4-27 gives a closer picture of the wave packet evolution.

The system changes from convectively unstable to absolutely unstable when the large-scale flow becomes stronger. In this barotropic model, the mechanism is simple: the propagation of the modes with smaller group velocity than the maximum velocity of the large-scale flow is arrested or even retrograded. The ray $y / t=c_{g}$ becomes closer to the ray $y / t=0$ around the area where the large-scale flow velocity reaches the maximum, because $c_{g}$ of the wave packet is affected by the meridional velocity adjustment and is possible to become zero. We calculate the local growth rate $\omega_{i}$ and quantify this mechanism.

The local growth rate is calculated by a least square fitting of the time series 


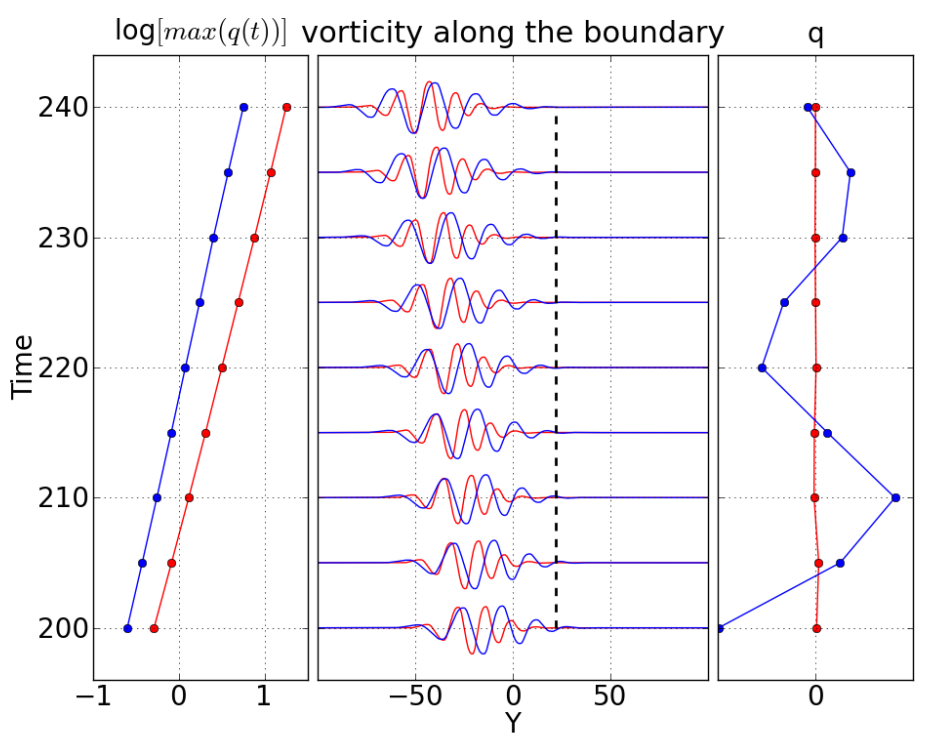

Figure 4-25: The time evolution of the streamfunction in the streamwise direction. Two cases are compared in this figure. The red lines represent the perturbation in the JOBG, while the blue lines show the result of the JWBG. The jet is represented by a half-Bickley jet with the maximum velocity 1 flowing southward. The strength of the gyre structure is represented by the maximum of the streamwise velocity, $\bar{v}_{2 \max }=0.1833$ in this case. The left panel shows the logarithm of the maximum of the streamfunction in the streamwise direction, which can be used as an index for the growth of the perturbation. The middle panel shows the time evolution of the streamwise structure of the streamfunction. Each line is normalized by its absolute maximum. The traveling (convective) features of the perturbation are obvious. The right panel shows the local perturbation variation in time at the location indicated by the black dashed line in the middle panel. The local signal decays slower for JWBG case (one should be aware that the location is upstream where the jet is retarded by the large-scale flow).

of local streamfunction $\psi(t ; y)$ to a decaying or growing sinusoidal wave $\widehat{\psi}(t ; y)=$ $A e^{\omega_{i}^{\ell} \mathrm{t}} \sin \left(\omega_{r} \mathrm{t}+B\right)$. The parameter $\omega_{i}^{\ell}$ gives the local growth rate, and $\omega_{r}$ gives the frequency. Figure 4-28 is an example of the best fit of both the growing and the decaying signal, showing that the local wave behavior is well described by the target function $\widehat{\psi}(t ; y)$.

The least square fitting described above is used to examine the local growth rate at different streamwise stations. The parameters are calculated by examining the 


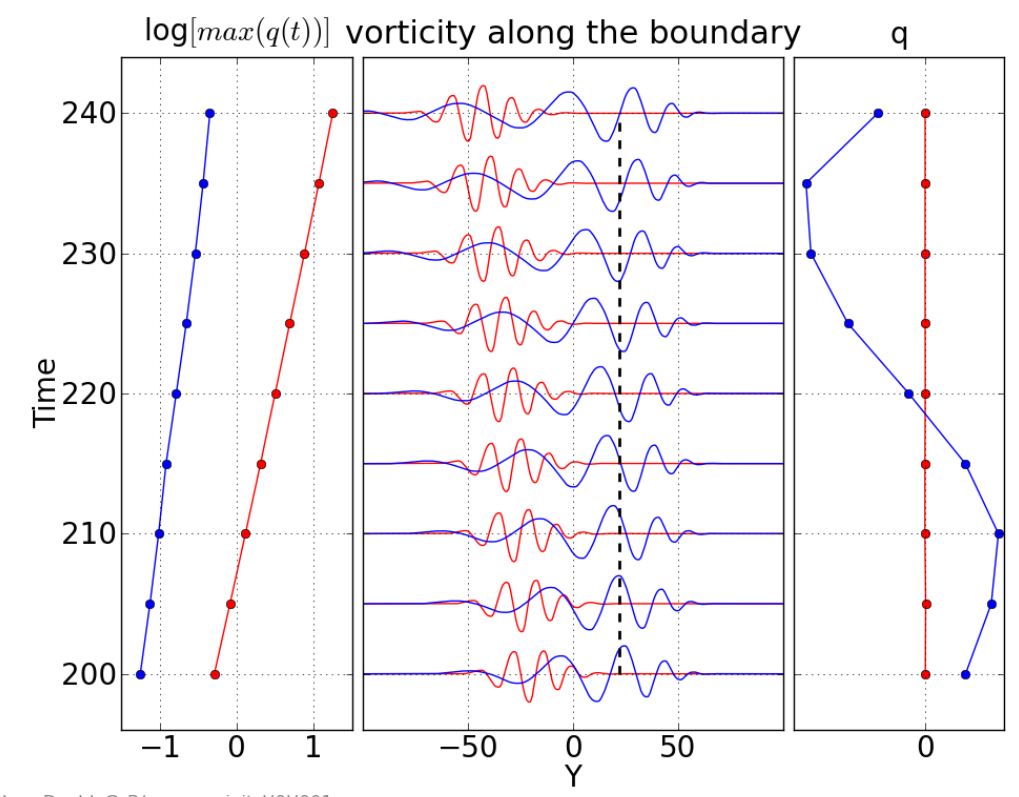

Num DoubleG B/p gauss init Uouo01.py

Figure 4-26: The same as in Fig 4-25 but for a stronger large-scale flow. $\bar{v}_{2 \max }=0.477$ in this case. The non-uniform structure is more obvious in this figure. The signal is stretched when traveling downstream. At the fixed station, the instability is switching from convective to absolute (the growing blue curve).
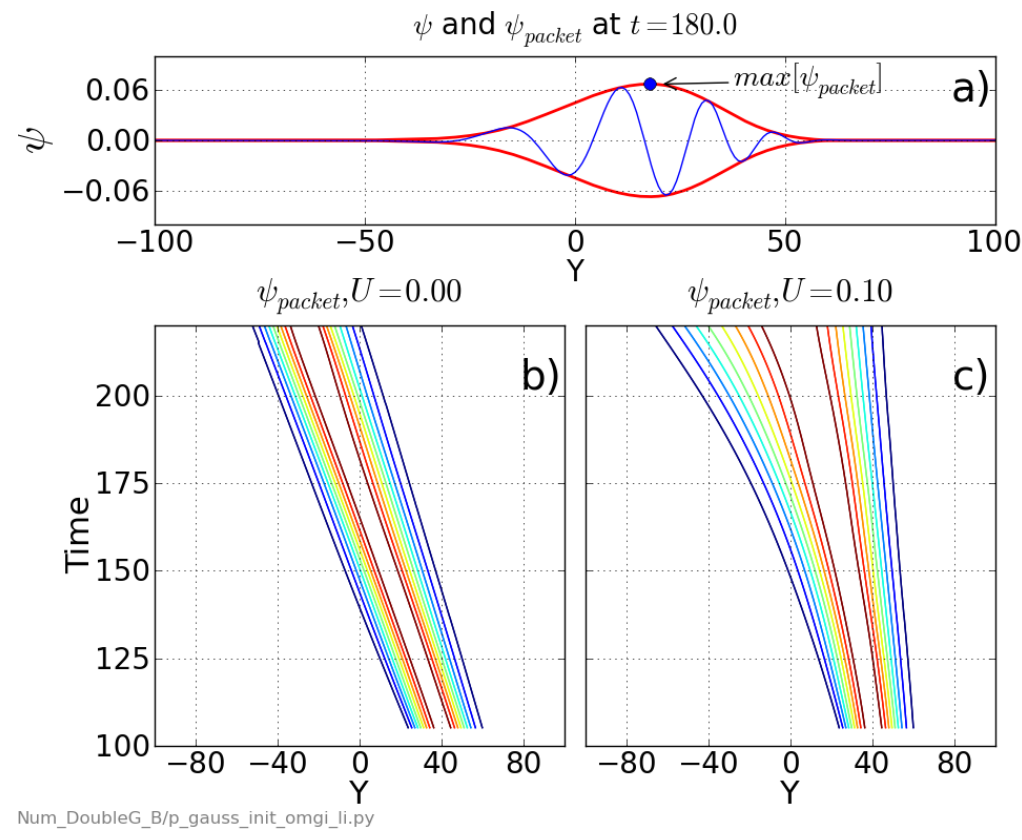

Figure 4-27: The same as Figure 4-18 but for a gyre large-scale flow. The wave packet spreads more at the front side than the rear. 


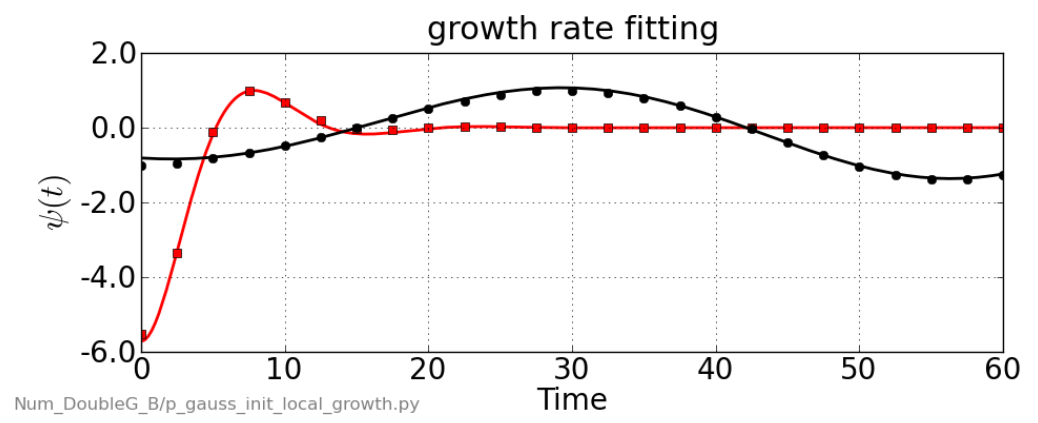

Figure 4-28: The growth rate and frequency are retrieved by least-square fitting of a local streamfunction (dots) to $\widehat{\psi}(t ; y)=A e^{\omega_{i}^{\ell} \mathrm{t}} \sin \left(\omega_{r} \mathrm{t}+B\right)$ (solid lines). The black color shows the growing signal, and the red color shows the decaying signal. The signal is rescaled for the purpose of illustration.

local behavior at a particular station, say $y_{0}$, after a Gaussian perturbation centered around $y_{0}$ is initialized. In other words, each simulation gives us a pair of parameters $\left(\omega_{i}^{\ell}, \omega_{r}\right)$ at location $y_{0}$, with $y_{0}$ shifting from $-W_{y} / 2$ to $W_{y} / 2$.

Figure 4-29 shows the results of the fitting. The local response to the initial Gaussian perturbation follows the structure of the meridional velocity of the gyres. The group velocity and the phase speed will be reduced over the region $y>0$, where the large-scale flow is against the wave propagation. What an observer sees over that region is that the oscillation becomes slower, and the local frequency becomes smaller. The behavior is opposite for the region where the large-scale flow accelerates the jet $y<0$. This process is closely related to the effect of the meridional velocity adjustment, so that the meridional structure of the local frequency shows the structure of the meridional velocity of the large-scale flow. For the same reason, the local growth rate also follows that structure.

Note that the effect of the meridional velocity adjustment, discussed in the WKB analysis is not the only influence. There are small discrepancies at $y=0$ for the values with different large-scale flow intensities. Those differences are caused by the zonal advection of the perturbation PV by the large-scale flow. The influence of the zonal PV advection is smaller compared to the change by the effect of meridional PV 


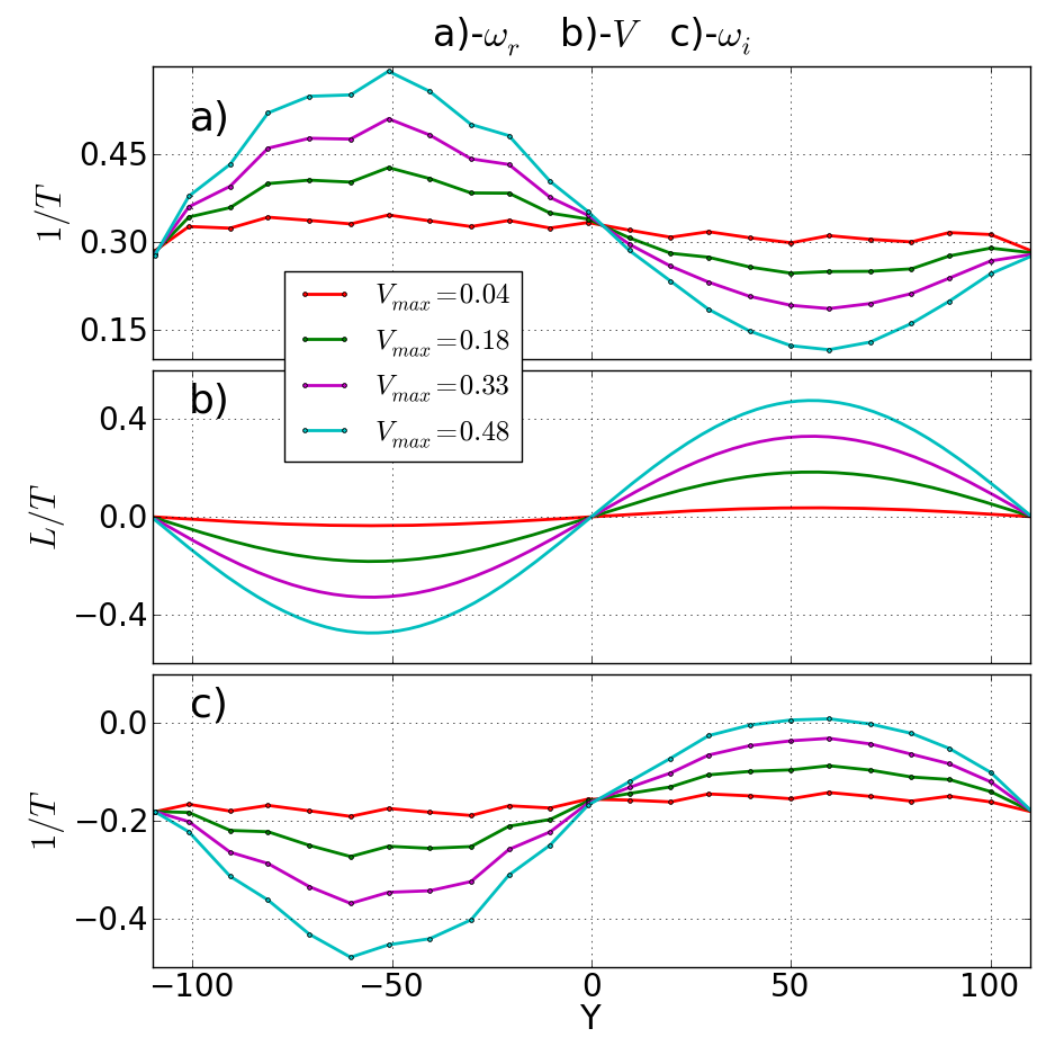

Num_DoubleG_B/p_gauss_init_local_growth.py

Figure 4-29: Results of the least square fitting of the local response to a pulse perturbation within the boundary region. (a) shows the frequency, (b) shows the meridional velocity of the gyres along the boundary, (c) shows the growth rates whose positive value corresponds to growing signals and vice verse. The color indexes are the same for all panels, and labeled in the legend. Each color refers to a different gyre strength.

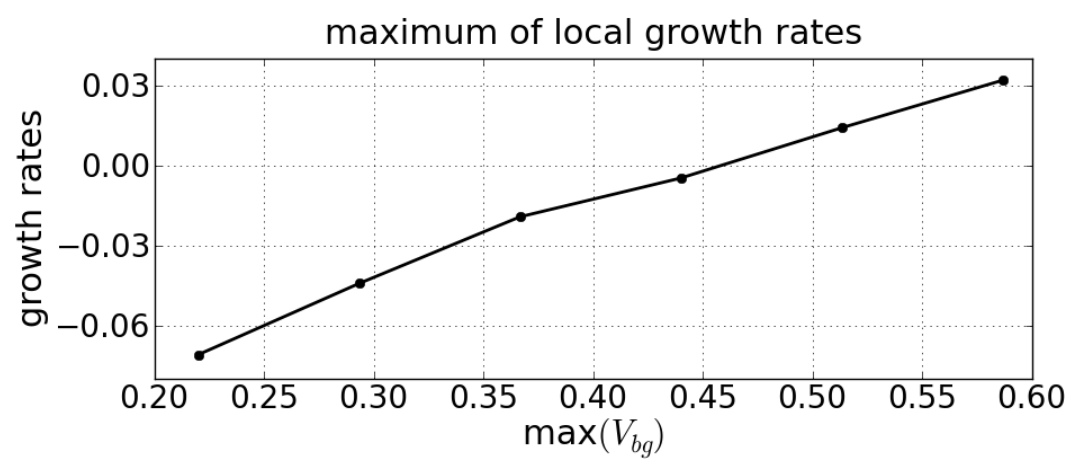

Figure 4-30: The growth rate as a function of the maximum meridional velocity of the large-scale flow along the boundary. It switches sign at about $\bar{v}_{2 \max }=0.46$. 
advection.

When the intensity of the gyres becomes large enough, for example $\bar{v}_{2 \max }=0.48$ in the figure, the local growth rates for the region, where the gyre flow is most strongly against the wave propagation, switch sign from negative to positive. It means that the absolute instability starts to emerge. Figure 4-30 shows the maximum local growth rate as a function of the associated local meridional velocity of the large-scale flow. The sign-switching of the growth rate happens at around $\bar{v}_{2 \max }=0.46$. The global mode may merge as the absolute instability is a necessary condition for the global mode.

\section{Global mode}

A global mode emerges once there is an absolutely unstable region. Figure 4-31 shows the emergence of the global mode as the gyre becomes stronger. The model is initialized by a Gaussian perturbation indicated by the blue lines, integrated for a sufficiently long period for the global mode to start to emerge. Four examples are shown for a qualitative discussion of the behavior of the global mode. When $\bar{v}_{2 \max }=$ 0.66 (panel a), the jet supports absolute instability according to previous results. The wave packet is marching slowly forward. The final state is quasi-stationary, which means the wave packet moves at a very slow speed. When the gyre becomes stronger, $\bar{v}_{2 \max }=0.73,0.81$, the global mode forms enveloping the standing waves $(\mathrm{b}, \mathrm{c}, \mathrm{d})$. The peak of the global mode shifts northward following the increase of the large-scale flow intensity (compare b with $\mathrm{d}$ ). The global mode does not depend on where the perturbation is initialized (compare b and c). The units of the streamfunction are the same in all panels. The growth rate of the global mode decreases following the increase of the gyre strength (compare a, b, d). The large-scale flow intensity is the same in panel b and c. However, because of the longer development time, the perturbation in panel $\mathrm{c}$ has a larger amplitude than that in panel b.

It is shown that the influence of the gyre on the eastern boundary jet instability 


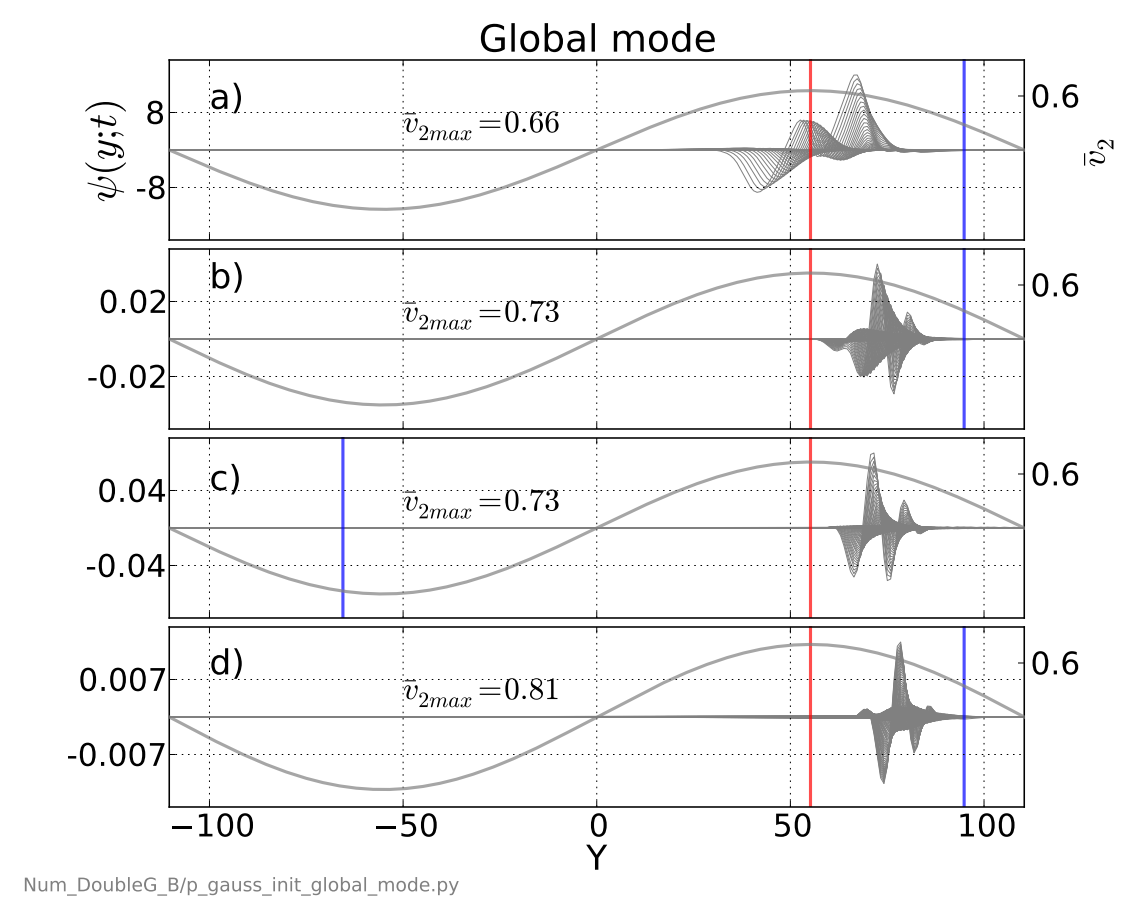

Figure 4-31: Global modes for different gyre strengths. The thick gray curves show the meridional velocity along the boundary with the maximum velocity $\bar{v}_{2 \max }$, whose values are shown by the label and the location marked by red lines. The blue lines indicate the locations where the initial Gaussian perturbation is launched. The thin gray curves show the snapshots of the stream-function at successive time steps. The pattern formed by those stagnant wave packets is defined as the global mode.

is similar to the influence of the partial-gyre flow in terms of altering wave speed and stretching the wave packet. The global mode emerges essentially because the retrograde large-scale flow is strong enough to arrest at least part of the wave packet, so that the local growth rate switches from negative to positive and forms an absolute instability. The large-scale flow slows down the convective behavior of the unstable mode to form a global mode. Thus far we discuss the behavior of the system with a fixed $\beta$. Next we consider two cases with different $\beta$ s and examine the $\beta$ effect.

Increasing $\beta$ has two important effects on the eastern boundary jet instability: the declining of growth rates and the decreasing of phase speeds for all unstable modes. As mentioned previously, the relative strength of the velocity of the large-scale flow to the wave (phase/group) speed is an important index for emergence of the global mode. Although the mechanisms are still unclear, the beta effect reduces the phase 
speed of the signal, but not the group velocity. For gyres with the same intensities, the phase speeds are different in cases with different $\beta$, but the envelope of the global mode does not change as the group velocity does not depend on $\beta$. Figure 4-32 confirms the idea that for a smaller $\beta, 0.02$ in this case, the initial perturbation evolves and travels downstream and becomes stationary after a sufficiently long time $t \sim 300$. As $\beta$ becomes larger, to 0.05 , the phase line of the signal travels in an opposite direction but the peak of the envelop does not change (it is more obvious in the following quantitative description). The growth rate is reduced in the large $\beta$ case (left panel).

The beta effect has no influence on the location of the global mode, but does reduce the growth rate of the unstable mode, as it does to the parallel boundary jet.

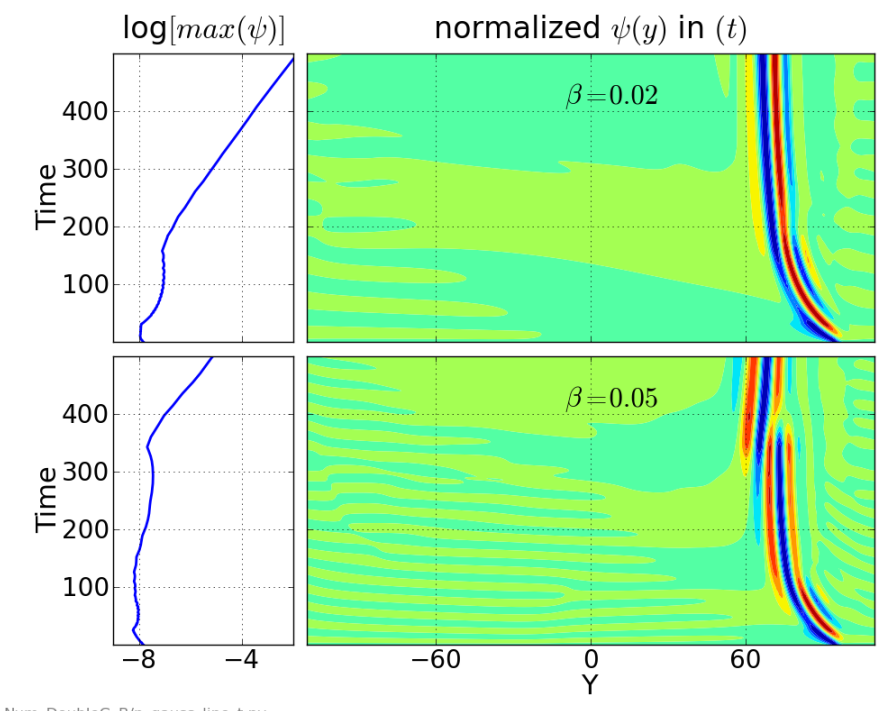

Figure 4-32: The $\beta$ effect. Right panels show the Hovmöller diagram of the along boundary streamfunction normalized by the maximum value at each time step. The jump at $t=400$ in the right panel is an artifact brought by the normalization. When $\beta$ is smaller, 0.02 in this case, the initial perturbation evolves and travels downstream and becomes stationary after a sufficient long time $t \sim 300$ (upper). When $\beta$ becomes larger, the signal turns stationary faster. The phase travels in the opposite direction afterward (lower). 


\section{Global mode from a random noise initialization}

The relationship between the large-scale flow and the global mode is further tested with a focus on the location of the peak of the global mode. In this experiment, we use four gyres instead of two gyres as the large-scale flow. Sponge layers are applied to all boundaries except for the eastern boundary. Two sponge layers at the northern and southern boundary are used to prevent the outgoing signal across the southern boundary from reentering the domain through the northern boundary. If the whole system is convectively unstable, perturbations eventually propagate out of the middle double-gyre region, and are damped out over the sponge layer region, resulting in a

quiescent system. The sponge layer at the western boundary is used to damp out the wave reflection. Figure 4-33 shows the model setup with the illustration of the gyres, the sponge layers, and the boundary jet. The domain size is 30 in the zonal direction and 250 in the meridional direction, again in the non-dimensional form scaled by the width of the boundary current. The spatial resolution is 256 by 256 grid points in the zonal and meridional direction, and the nondimensional time step is 0.125 . The model is initialized by small random noise within the jet, and integrated to $t=500$. A global mode can emerge if the system supports an absolute instability.

A series of experiments are run with the same strength of the boundary current but varying strengths of the large-scale flow. Consistent with the previous section, the maximum meridional velocity of the large-scale flow along the eastern boundary $\bar{v}_{2 \max }$ is used as the index measuring the large-scale flow strength. In this series of experiments, $\bar{v}_{2 \max }$ varies from 0.04 to 0.83 . The global mode, if there is one, is represented by a third-order spline fit of the meridional structure of the streamfunction. An example can be found in Figure 4-27. The location of the peak of the global mode is denoted as $y_{p}$. For each specific large-scale flow, two experiments with $\beta=0.01$ and 0.02 are carried out to investigate the beta influence on the global mode.

Figure 4-34 shows the results. Black color indicates the case with $\beta=0.01$, and the red color indicates the case with $\beta=0.02$. Symbols represent the numerical 


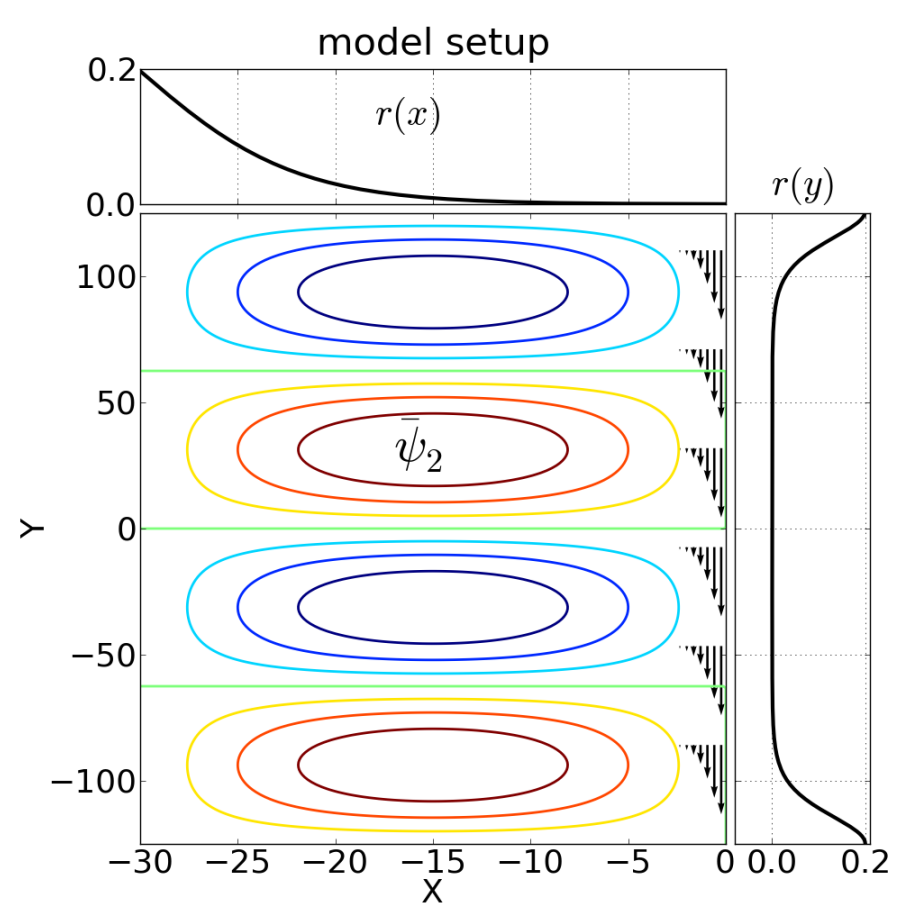

Figure 4-33: The model setup for simulations with random noise initializations. The model is the same as the one in Figure 4-23 but with four gyres and three sponge layers (the top and right panel).

results, and lines are the linear regression of the numerical results. The location of $\bar{v}_{2 \max }$ is denoted as $Y_{M}$ and is represented by the dashed line. It is a fixed value for all experiments as the structure of the large-scale flow is unchanged.

Many features coincide with previous mechanisms. We notice from the numerical results (the symbols) for both cases (red and black) that no global modes are supported when the maximum meridional velocity of the large-scale flow is less than about 0.46 , the same value shown in Figure 4-30 at which the absolute instability first emerges.

The relationship between $\bar{v}_{2 \max }$ and $y_{p}$ is approximately linear for both values of $\beta$ , which can be inferred from the linear best-fits of the numerical results (red and black lines). The positive slope means that an increase of the intensity of the large-scale flow leads to a northward shift of the peak of the global mode. It can be explained by the influence of the meridional PV advection, the effect of the meridional velocity 


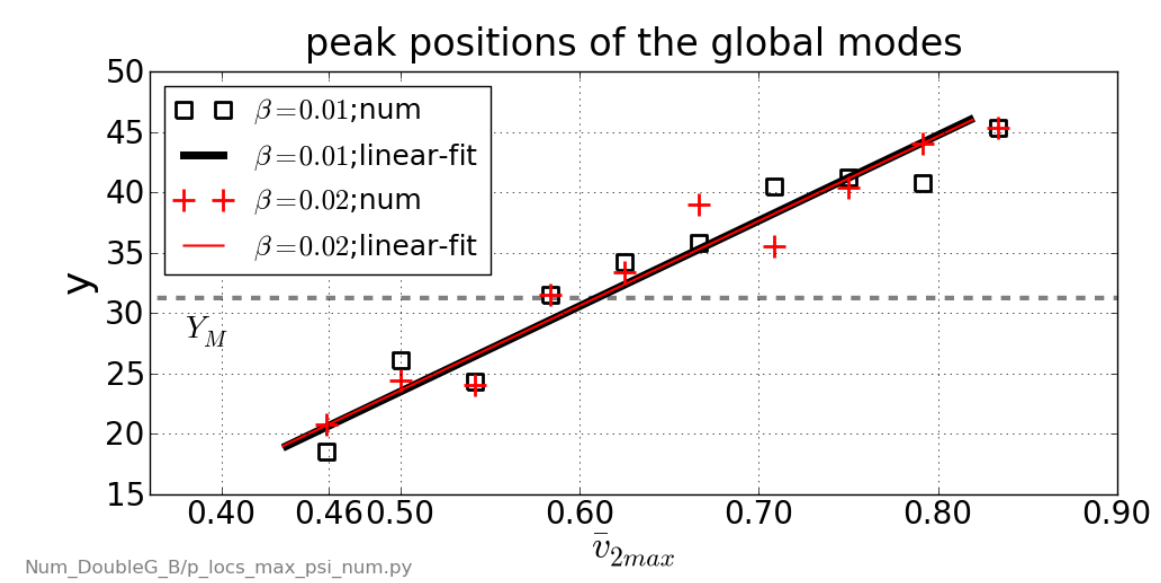

Figure 4-34: The relationship between the location of the peak of the global mode $y_{p}$ and the maximum meridional velocity of the background flow $\bar{v}_{2 \max }$. The envelope of the global mode is represented by a third-order polynomial. Their peak locations are shown by the symbols (black squares and red crosses) for each experiment with different $\bar{v}_{2 \max }$ and $\beta$. The solid red and black lines are the linear best fit of the symbols. Two betas are considered, $\beta=0.01$ (red) and $\beta=0.02$ (black).

adjustment.

The slopes of the linear fit in the two cases coincide with each other as shown by the red and black solid lines. This means that the relationship depends on the group velocity instead of the phase speed, because $\beta$ alters the latter but not the former. Figure 4-35 shows the group velocity for the case with $\beta=0.01$ (black) and $\beta=0.02$ (red). $\beta$ does not change the group velocity (compare the red and the black dashed line), except at the long wave end where long wave modes can be stabilized by increasing $\beta$. The differences over the short wave end are suspected to be caused by numerical noise. The short wave modes are less important in the global mode structure as they have very small growth rates.

The location of the peak of the global mode $y_{p}$ crosses the location of the maximum northward velocity $Y_{M}$ at about $\bar{v}_{2 \max }=0.61$. This means the peak of the envelope of the global mode is located downstream (the boundary current flows southward) of $Y_{M}$ for $\bar{v}_{2 \max } \lesssim 0.61$, and vice versa. What process sets the value? We notice that the minimum absolute group velocity of the unstable modes show in Figure 4-35 is 


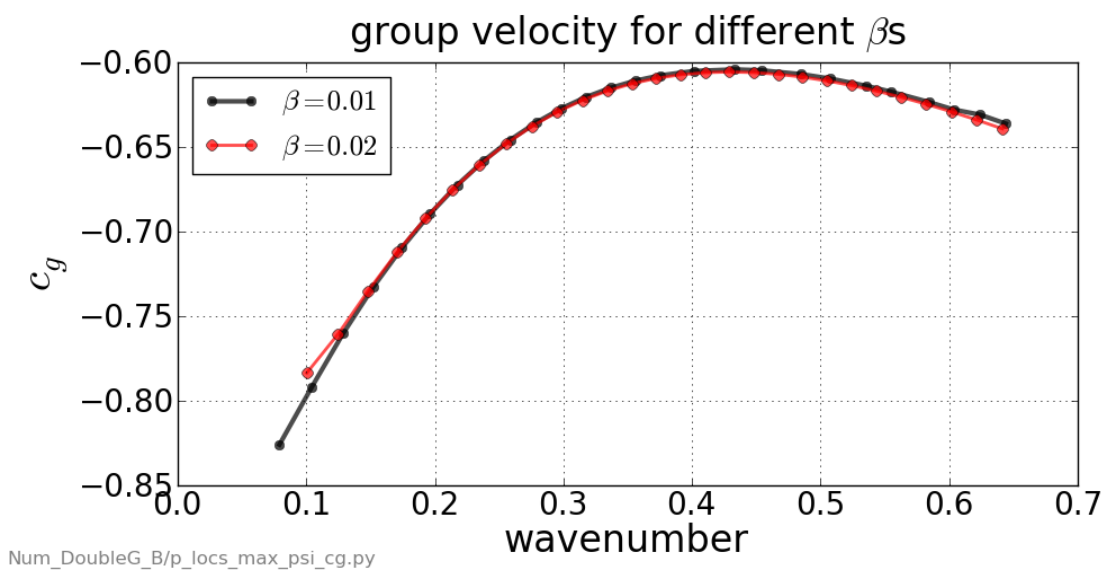

Figure 4-35: The group velocities in two cases with $\beta=0.01$ (black) and $\beta=0.02$ (red). The group velocity is calculated using the same method used in Figure 4-11.

also around 0.61 . This indicates that for large-scale flows with $\bar{v}_{2 \max } \gtrsim 0.61$, more unstable modes are arrested north of $Y_{M}$ to form the local peaks, but for large-scale flows with $\bar{v}_{2 \max } \lesssim 0.61$, even the slowest mode (in terms of group velocity) can leak energy downstream of $Y_{M}$ and the peaks of the global mode are also downstream. This is similar with what is found in Pierrehumbert (1983) where the peak of the global mode is found downstream of the maximum baroclinicity in a two-layer baroclinic model.

\subsection{Conclusion and discussion}

In this chapter, the influence of the large-scale flow on the instability of an eastern boundary jet is studied. Two types of large-scale flows are considered, a partial-gyre flow and a double-gyre flow. The partial-gyre flow has a simple structure and is studied using the WKB method. A linear numerical model is also used to validate the WKB solution. Results from the two methods agree with each other within a $\% 5$ tolerance range. The double-gyre flow is studied using the numerical model. Its influence can also be explained by the mechanisms proposed in the partial-gyre flow study.

The influence of the large-scale flow has two components. One is related to the 
zonal transport of the perturbation PV by the large-scale flow, which induces a stabilizing effect if the zonal transport is onshore. The second component is related to the meridional transport of the perturbation PV by the large-scale flow. It affects the instability mainly through the meridional velocity adjustment. The non-uniformity of the large-scale circulation yields the meridional structure of the instability through accelerating or retarding the signal. A global mode is established with a peak at a preferred location. The location is determined by the relative strength of the large-scale flow and the group velocity of the perturbations.

In the WKB analysis, the zonal transport of the perturbation PV by the partialgyre flow contains two parts, $\bar{u} v_{x x}$ and $-\bar{u} u_{y y}$. The former has stabilizing effect, and the latter resembles the $\beta$ effect, altering the local temporal growth rate and the phase speed of the perturbation. The positive zonal velocity produces similar wave behavior as produced by a $-\beta$, which is indicated by the effective $\beta: \beta-F \ell^{2}$. An increase of intensity of the large-scale on-shore flow is equivalent to a decrease in $\beta$, which leads to an increase in the phase speed. The destabilizing effect caused by this effective $\beta$ is overcome by the stabilizing effect of $u v_{x x}$, so that the zonal advection of the perturbation PV by the mean flow stabilizes the boundary jet.

The meridional PV advection leads to mainly the meridional adjustment of the group velocity of the signal. The group velocity is raised where the large-scale flow is southward, and reduced when the large-scale flow is northward. The altered group velocity can affect the spatial growth rate $\ell_{i}$ through $\ell_{i} \simeq \omega_{i} / c_{g}$. This mechanism is confirmed by an examination of the relationship $\ell_{i} \simeq \omega_{i} / c_{g}$. Over the region where the jet is accelerated, the perturbation $\psi=\phi(x ; t) e^{\mathrm{i} \ell y}$ is elongated in both the meridional and the zonal direction, and the spatial growth rate is reduced. The opposite is true over the regions where the jet is slowed down.

These two mechanisms proposed in the WKB analysis are also confirmed by a series of numerical simulations. The neglected terms in the WKB analysis have a minor effect on the overall conclusion.

The double-gyre flow is studied using a pulse instability and a random noise ini- 
tialization. Using the pulse instability, we found that as the large-scale circulation becomes stronger, the boundary jet switches from convectively unstable to absolutely unstable over the region where the large-scale circulation is strongly against the propagation of the signal (referred to as $Y_{M}$ ). The global mode appears after the system acquires an absolutely unstable region. A qualitative study of the $\beta$ effect on the global mode using the pulse instability shows that the phase speed is changed by the $\beta$ effect in the same way shown by the eigenvalue solutions, but the global mode is unchanged, which is confirmed by a quantitative study later using the random noise initial condition. The system becomes less unstable when the large-scale flow becomes stronger, which is consistent with the results of the partial-gyre flow.

A random noise initialization is used to study the general relationship between the large-scale circulation and the global mode. The maximum of the meridional velocity $\bar{v}_{2 \text { max }}$ instead of the zonal inflow $\bar{u}_{2}$ is used as the index of the large-scale circulation, because the former is easier to compare with the meridional group velocity of the unstable modes. Two sponge layers at the northern and southern sides are used to prevent energy recycling. The global mode emerges after a sufficiently long time integration if the parameters match certain conditions.

One of the conditions concerns the minimum meridional velocity of the large-scale flow needed for the absolute instability to appear. It is confirmed by the simulations with random noise initializations that no global mode exists for the large-scale circulation with $\bar{v}_{2 \max }$ less than 0.46 , which is the velocity threshold of the large-scale flow for the absolute instability to occur.

Once the large-scale circulation is strong enough so that the system supports the absolute instability at least over some regions, the position of the peak of the global mode has a linear dependence on $\bar{v}_{2 \max }$. If the large-scale circulation can arrest part of the unstable mode, the peak is more likely located north of $Y_{M}$. If the group velocity for all unstable modes is larger than $\bar{v}_{2 \max }$, the peak is located downstream (south) of $Y_{M}$. This downstream shift is also observed in the two layer baroclinic model of Pierrehumbert (1984). 
Another interesting feature is that $\beta$ does not alter the group velocity although it does change the phase speed of the unstable mode. The location of the peak of the global mode is intimately linked to the group velocity, so that the $\beta$ effect does not change the relationship between the peak location and the intensity of the largescale flow. It has been found in both previous studies and this study that the beta effect changes the phase speed of unstable modes of a meridional current. However, the mechanism for this feature is still unclear. It is also possible that the changes in group velocity is too small to be detected in the parameter range used in this chapter. It is important for future study to investigate this relationship between the phase speed and beta, with which the relationship between the group velocity and beta is expected to be easily derived.

The discussion presented in this chapter remains in a linear framework, which is an obvious limitation. The WKB solution is valid only under the assumption that $\epsilon \ll \mathcal{O}(1)$. The numerical simulations studied in this chapter fall also under this assumption. The linear combination of the $\beta$ effect and the effect of the background flow may change if the background flow becomes intense or $\beta$ becomes large. Can the global mode emerge in a linear regime, or be distorted by the nonlinear interactions before it can form? Can we find the linear signatures in the nonlinear simulation? Those questions will be addressed in the next chapter where the nonlinear interaction is considered and the linear theory is tested. 
THIS PAGE INTENTIONALLY LEFT BLANK 


\section{Chapter 5}

Instability of an eastern boundary current influenced by a large-scale flow: a nonlinear study 


\section{$5.1 \quad$ Introduction}

This chapter combines the model components considered in previous three chapters, and discusses their interactions based on nonlinear numerical simulations. Chapter 3 demonstrates that there are two types of unstable modes, the linearly unstable trapped mode and the nonlinearly unstable radiating mode. Chapter 4 shows that an unstable wave packet is subject to the influence of a large-scale flow. This chapter investigates how a large-scale flow influences the instabilities of an EBC. Does the large-scale flow impose the same or different influences on the trapped mode and the radiating mode? What are the characteristics of the affected unstable modes?

This chapter is organized as follows. Section 5.2 describes the numerical model and the forcing field. Section 5.3 lists the experiments and their parameters. Results are described in Section 5.4, and the mechanisms are explained in Section 5.5. Section 5.6 presents the conclusion and discussion.

\section{$5.2 \quad$ Model}

\subsubsection{The vorticity equation}

This chapter makes use of the barotropic model on a beta plane used in Chapter 3, but with a different forcing field. The model equation is:

$$
\frac{\partial}{\partial t} \nabla^{2} \psi+J\left(\psi, \nabla^{2} \psi+\beta y\right)=\mathcal{F}+\nabla \cdot A_{h} \nabla \nabla^{2} \psi
$$

The associated numerical method is described in Appendix A.

\subsubsection{Forcing field}

The boundary current is set up following the same procedure extensively discussed in Section 3.2.3. The basic flow is driven by a prescribed forcing field, which can be calculated following Sverdrup relation $\mathcal{F}_{b}=-\beta V_{b} \operatorname{sech}^{2}\left(\frac{x-x_{c}}{L_{x b}}\right) . \mathcal{F}_{b}$ has the same units 
with the time tendency of vorticity, $s^{-2}$.

The large-scale flow can be properly represented by a double-gyre structure because it resembles the ocean gyres, which also satisfies the north-south periodic boundary conditions. It can be forced by an Ekman pumping (or equivalently a wind-stress curl) of a sinusoidal form in $y, \mathcal{F}_{g}=F_{g} \sin \left(2 \pi y / L_{g}\right)$. The underlying mechanisms are well understood (Stommel, 1948; Munk, 1950; Pedlosky, 1987). Such a forcing structure can produce a double-gyre with a cyclonic gyre in the northern basin and an anticyclonic gyre in the southern basin.

The total forcing field is then represented by

$$
\mathcal{F}=\mathcal{F}_{b}+\mathcal{F}_{g}=-F_{b} \operatorname{sech}^{2}\left(\frac{x-x_{c}}{L_{x b}}\right)+F_{g} \sin \left(\frac{2 \pi y}{L_{g}}\right)
$$

where $x_{c}$ represents the location of the center of the boundary current.

The instability of the boundary current becomes much more complex once the influence of a steady large-scale flow is considered. More complexity can be added into the system by an unstable large-scale flow. The interaction between an unstable boundary current and an unstable large-scale flow is a very interesting topic but less relevant to our focus, so that we keep the large-scale flow in a stable regime. The model is forced by a weak forcing field and damped by a western-intensified lateral friction.

The zonal structure of the lateral eddy viscosity coefficient $A_{h}$ is

$$
A_{h}=\left(A_{h}^{W}-A_{h}^{E}\right) \exp \left(\frac{-x}{0.15 L_{g}}\right)+A_{h}^{E}
$$

where $A_{h}^{W}$ and $A_{h}^{E}$ represent the coefficients along the western boundary $(x=0)$ and approximately the eastern boundary $\left(x=L_{g}\right)$. One example of the zonal structure of $A_{h}$ is shown in Figure 5-1 with $A_{h}^{W}=10^{4} \mathrm{~m}^{2} / \mathrm{s}$ and $A_{h}^{E}=10^{2} \mathrm{~m}^{2} / \mathrm{s}$, which will be used in this study unless they are explicitly specified. The western-boundaryenhanced viscosity is intended to parameterize the boundary eddy-related processes, which are studied in detail in Fox-Kemper (2003), and to stabilize the large-scale 
flow. The small viscosity along the eastern boundary is used to parameterize the unresolved eddy dissipation, and also damp the numerical noise without eliminating the boundary current instability.

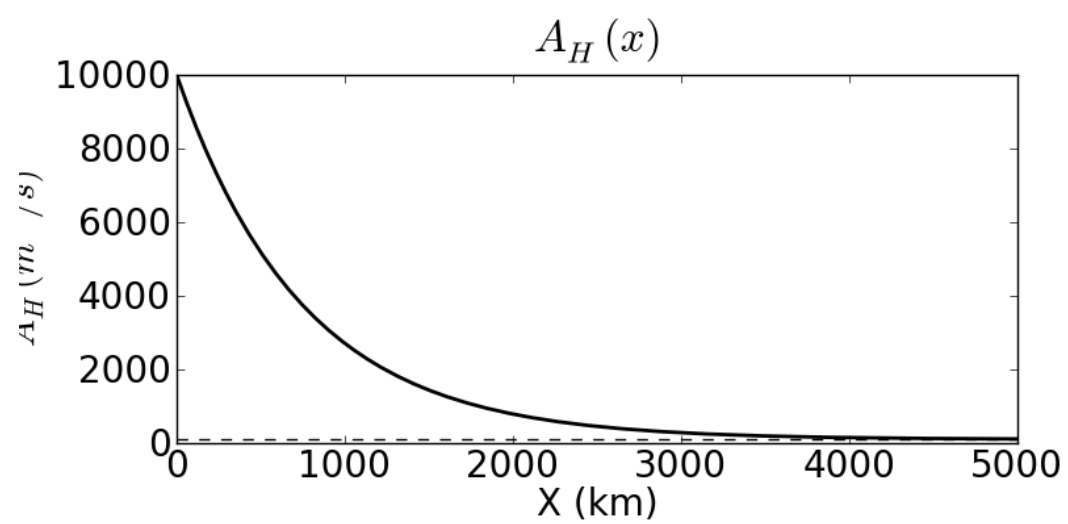

Figure 5-1: Horizontal viscosity coefficient varies from $10^{4} \mathrm{~m}^{2} / \mathrm{s}$ at the western boundary to $10^{2} \mathrm{~m}^{2} / \mathrm{s}$ at the eastern boundary.

Figure 5-2 shows the streamfunction of the forced gyres. The gyres are weakly forced and stay stable. The threshold for the gyres to transit from the stable regime to the unstable regime is not studied here.

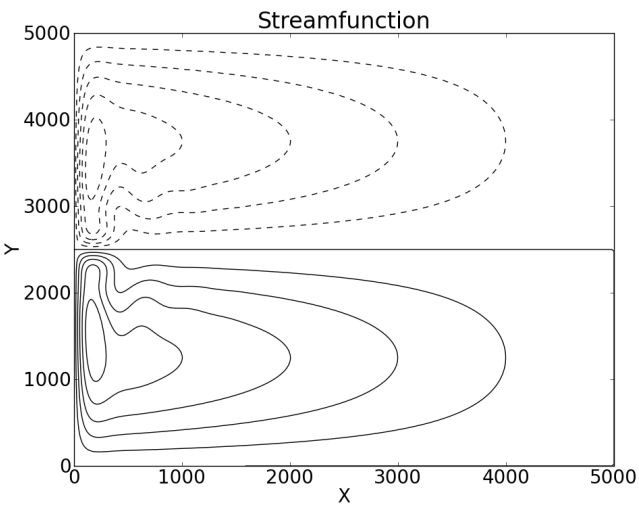

Figure 5-2: Snapshots of the streamfunction of a stable double-gyre at the end of the 30-year integration. The parameters are $F_{g}=3 \times 10^{-13} \mathrm{~s}^{-2}, \beta=2 \times 10^{-11}(\mathrm{~ms})^{-1}$, $A_{h}^{W}=10^{4} \mathrm{~m}^{2} / \mathrm{s}, A_{h}^{E}=10^{2} \mathrm{~m}^{2} / \mathrm{s}$. 

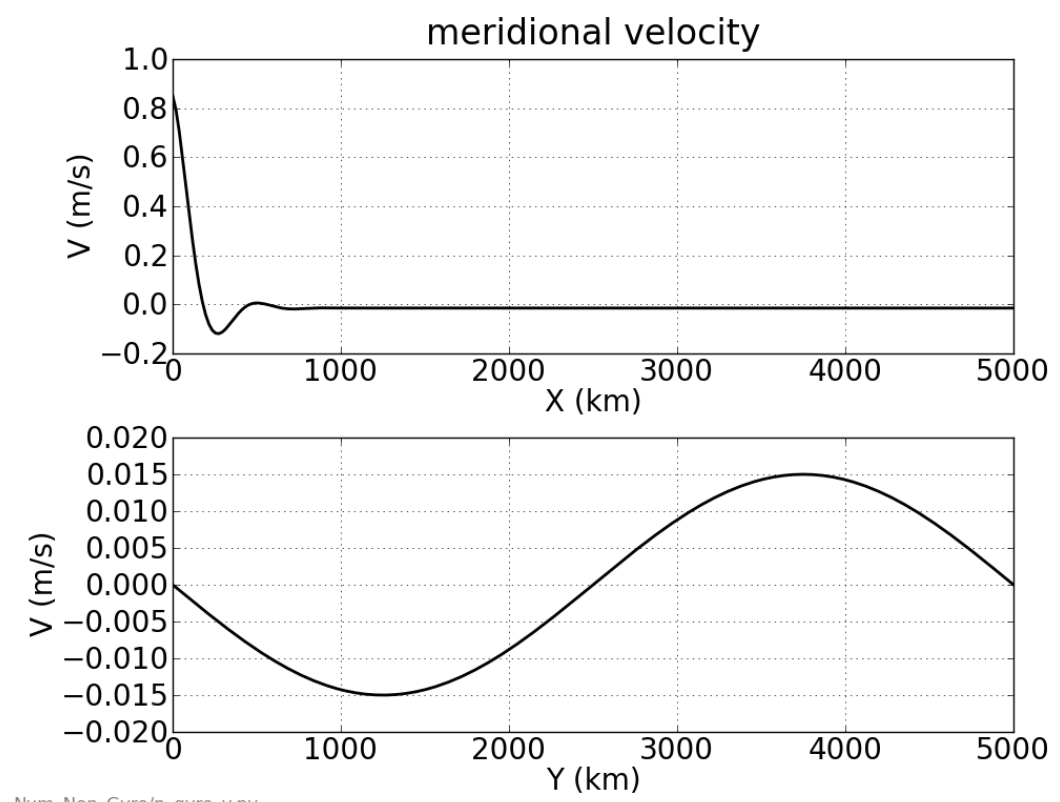

Num Non Gyre/p gyre v.py

Figure 5-3: The meridional velocity in the case shown in Figure 5-2. The upper panel shows the zonal structure of the meridional velocity at $y=1250 \mathrm{~km}$ and the lower panel shows the meridional structure along the eastern boundary. The maximum meridional velocity is about $0.83 \mathrm{~m} / \mathrm{s}$, which occurs along the western boundary because of the western intensification. The meridional velocity along the eastern boundary is about 1/50 the speed of the western boundary current.

Figure 5-3 shows the meridional velocity of the gyre in the stable case. The lateral friction dissipating the relative vorticity results in a Munk layer along the western boundary (Munk, 1950). The layer width is $\left(A_{h} / \beta\right)^{1 / 3}$, about $80 \mathrm{~km}$ in this case. The maximum of the meridional velocity along the eastern boundary is on the order of $10^{-2} \mathrm{~m} / \mathrm{s}$, about $1 / 50$ the speed of the western boundary current.

\subsection{Experiments}

We start from the model setups used in Chapter 3 and conduct a series of experiments and change various parameters, such as the forcing scale of the large-scale flow and of the boundary current. The model has a periodic boundary condition in the meridional direction and solid boundaries at the eastern and western sides with no-normal-flow 
and free-slip conditions. The domain is a square basin, $5000 \times 5000 \mathrm{~km}^{2}$. The grid is $256 \times 256$, which gives a horizontal resolution of $19.5 \mathrm{~km}$. Table 5.1 lists the experiments and their parameters. The parameters are within a reasonable range for a mid-latitude system. The forcing scale relates to the strength of Ekman pumping following the relation

$$
F=\frac{f}{D} w_{E},
$$

where $f$ is the Coriolis parameter, $D$ the layer depth, and $w_{E}$ the Ekman pumping velocity. the forcing scale $F_{g}=10^{-13} / s^{2}$ in case B25G100 (refer to Table 5.1) corresponds to an Ekman pumping velocity $0.12 \mathrm{~m} /$ day given $f=10^{-4} / \mathrm{s}$ and $D=400 \mathrm{~m}$. The meridional velocity relates to the forcing following the Sverdrup relation $\beta v=F$, so that given $F_{b}=2.5 \times 10^{-12} / s^{2}$ in case B25G000, the boundary current velocity scale is $V_{b}=0.125 \mathrm{~m} / \mathrm{s}$.

Table 5.1: List of experiments. $\beta$ is scaled by $10^{-11} / \mathrm{ms}$.

\begin{tabular}{|c|l|l|l|l|l|}
\hline Exp & $V_{b}(\mathrm{~m} / \mathrm{s})$ & $V_{g}\left(10^{-2} \mathrm{~m} / \mathrm{s}\right)$ & $L_{x b}(\mathrm{~km})$ & $x_{c}(\mathrm{~km})$ & $\beta$ \\
\hline B25G000 & 0.125 & 0 & 50 & 100 & 2 \\
\hline B25G025 & 0.125 & 0.125 & 50 & 100 & 2 \\
\hline B25G050 & 0.125 & 0.25 & 50 & 100 & 2 \\
\hline B25G075 & 0.125 & 0.375 & 50 & 100 & 2 \\
\hline B25G100 & 0.125 & 0.5 & 50 & 100 & 2 \\
\hline B30G000 & 0.15 & 0 & 50 & 100 & 2 \\
\hline B30G050 & 0.15 & 0.25 & 50 & 100 & 2 \\
\hline B30G075 & 0.15 & 0.375 & 50 & 100 & 2 \\
\hline B30G100 & 0.15 & 0.5 & 50 & 100 & 2 \\
\hline
\end{tabular}

In the next section, we use B25G000 and B25G100 as the standard simulations. Their comparisons are used to show the influence of the large-scale flow. Other experiments are used to explore the parameter dependence. 


\subsection{Results}

The nonlinear study in Chapter 3 shows that there are three phases in the evolution of a weakly nonlinear boundary current. The most unstable mode stands out from the initial random noise and saturates during phase one (PI hereafter); it stays in saturation during phase two (PII hereafter), and also resonates with its subharmonic to support the exponential growth of the subharmonic mode; during the phase three (PIII hereafter), the subharmonic mode stops growing, strongly interacts with the linearly unstable mode, and radiates significant energy into the interior.

Figure 5-4 shows the time evolution of the energy integrated over the eastern-half domain in case (BG25G000) without the large-scale flow. The energy plateaus clearly define three different phases, which are denoted by PI, PII, and PIII in the figure. PIII shows more energy because of the existence of the linearly stable but nonlinearly unstable longwave modes.

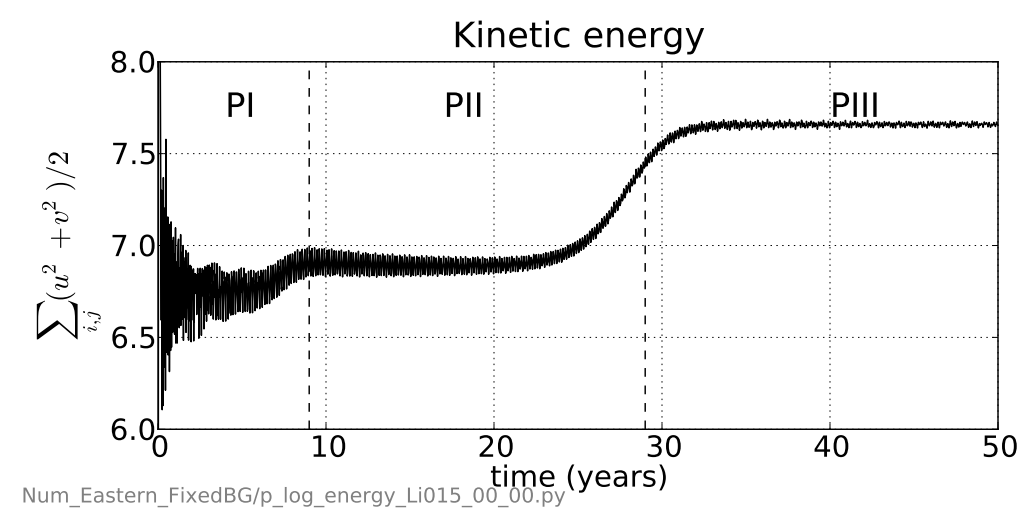

Figure 5-4: The time series of the energy integrated over the eastern-half domain in B25G000.

Different characteristics during different phases are also shown in the wavenumber spectra. The wavenumber spectra for two snapshots taken separately during PII (red) and PIII (black) are shown in Figure 5-5. The meridional wavenumber has units of $/ L_{g}$. The most unstable mode $n=15$, corresponding to a wavelength $333 k m$, is significant during both PII and PIII, but the longwave modes $n=7,8$ are only 


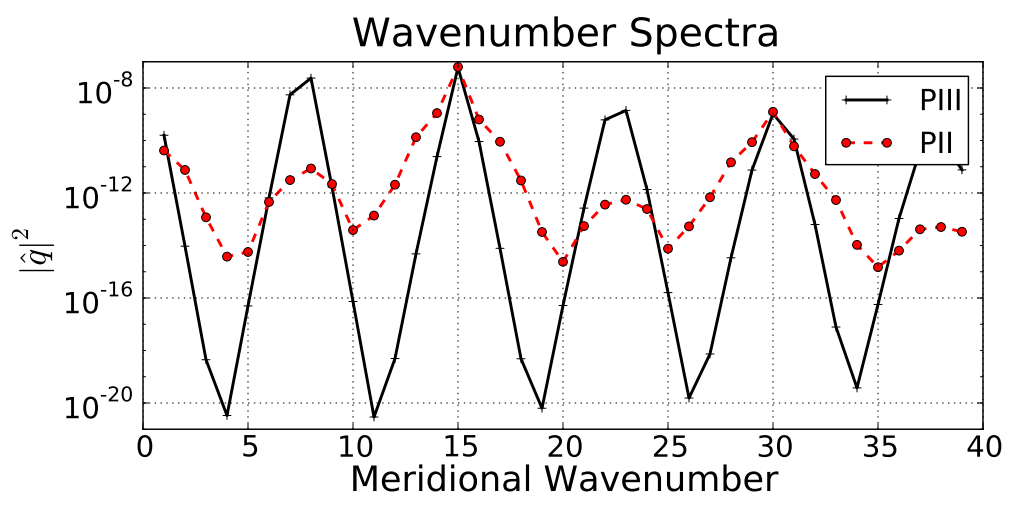

Figure 5-5: The wavenumber spectra of the vorticity field during the two phases : PII (red) and PIII (black). During PII, the most unstable mode is of $n=15$. During PIII, the most unstable mode barely changes, but the longwave modes with $n=7,8$ also appear. No subharmonic resonance is possible in this experiments as $n=7.5$ is not resolved. Instead, the nonlinear interaction occurs dominantly among a triad waves with $n=7,8,15$. The modes with larger wavenumbers, $n=22,23$ also become more energetic because of the triad interaction.

present during PIII. The longwave modes are driven by the nonlinear interaction, which is studied in Chapter 3. The time evolution of these three modes as shown in Figure 5-6 consolidates the mechanism of the triad interaction.

Figure 5-6 shows the evolution of three dominant modes. The red line represents the most unstable mode $n=15$. It exponentially grows during PI, becomes saturated during PII, and slightly changed during PIII. The longwave modes adjust during PI, start to grow exponentially during PII, and become equilibrated during PIII. One difference between this case, B25G000, and the one shown in Figure 3-19 is that the nonlinear interaction in this case occurs among a triad waves with three different wavenumbers, not between harmonics.

Figure 5-7 shows streamfunction snapshots during PII (left) and PIII (right) in B25G000. Perturbations are mostly trapped around the boundary region without efficient nonlinear energy transfer among modes during PII, but penetrates into the interior with the assistance of finite amplitude longwave modes. The background flow consists only of the parallel boundary current, so that the meridional wavelength of 


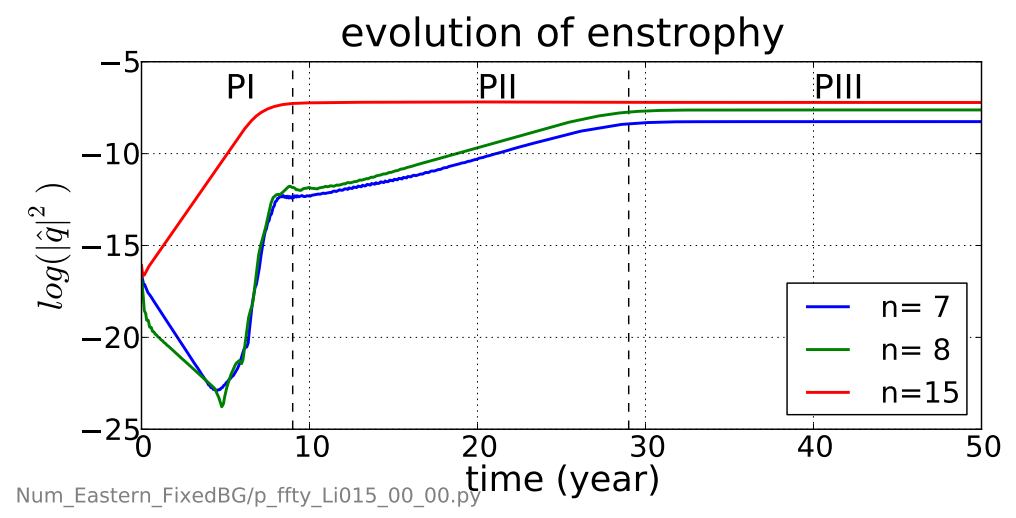

Figure 5-6: The time evolution of the power spectra of vorticity (equivalently enstrophy) for the modes $n=7,8,15$. Different phases distinctly show different characteristics in the enstrophy evolution. The most unstable mode grows and reaches equilibrium during phase one; stays in equilibration and drives the exponential growth of the longwave modes through nonlinear resonance during phase two; the system reaches equilibrium during phase three when the longwave modes stop growing.

the perturbation is uniform in $y$. It is not true for cases with the large-scale flow.
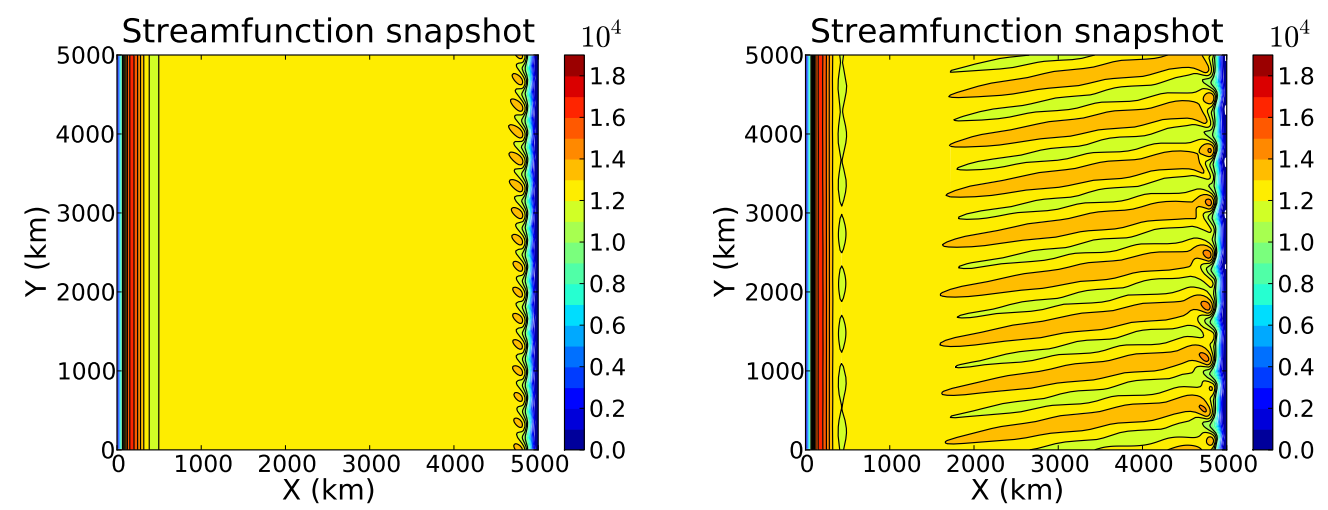

Figure 5-7: Streamfunction snapshots for case B25G000 during PII (left) and PIII (right).

Now we extend case B25G000 to case B25G100 by adding a double-gyre structure to the basic flow. The model parameters are listed in Table 5.1. The large-scale forcing field generates a similar double-gyre circulation as the one shown in the left panel of Figure 5-2. The influence of the large-scale flow on the instabilities of the boundary current is studied by comparing instability features in B25G100 with those 
in B25G000.

Figure 5-8 shows the time evolution of the kinetic energy integrated over the eastern-half domain in the case B25G100. The development in B25G100 is similar to the one in B25G000 (Figure 5-4), showing three different phases. The differences between these two cases are that the transition from PII to PIII is delayed approximately from year 27 in B25G000 to year 40 in B25G100, and that the final kinetic energy is smaller in B25G100 than that in B25G000.

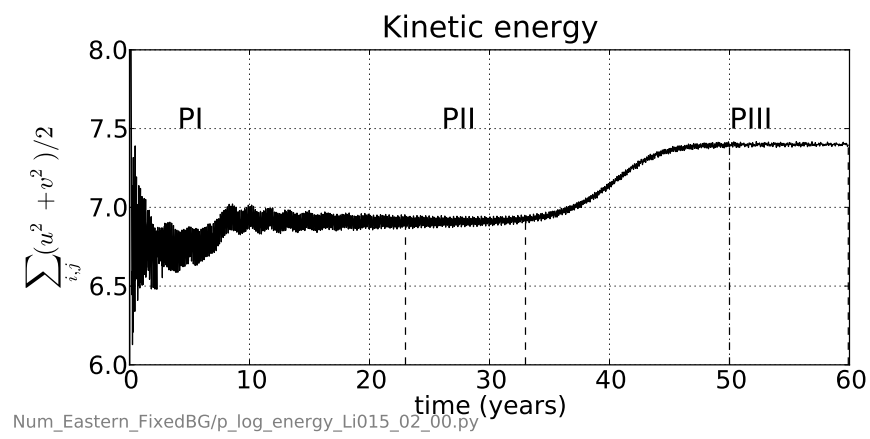

Figure 5-8: The time series of the kinetic energy integrated over the eastern half domain for case B25G100. The two segments indicated by the dashed lines are analyzed. The one between year 23 and 33 represents PII, and the one between year 50 to 60 represents PIII.

Figure 5-9 shows two streamfunction snapshots in B25G100. The left one is at year 30 during PII, and the right one is at year 55 during PIII. The double-gyre structure is clearly shown during both phases, but is distorted during PIII, when the longwave modes penetrate into the interior. The eastern boundary current is indicated by the sharp zonal gradient in the streamfunction field along the boundary.

We use eddy kinetic energy (EKE) as an index to show the changes between different phases and also between different cases. The time average is used to decompose the total field into the mean and perturbation components. The eddy kinetic energy is locally calculated as $0.5\left(u^{2}+v^{2}\right)$. Figure $5-10$ shows the results of the time-averaged EKE. The panel a1 shows the meridionally averaged EKE for the case without the gyres (B25G000) during PII (solid line) and PIII (dashed line). We use zonal profiles 

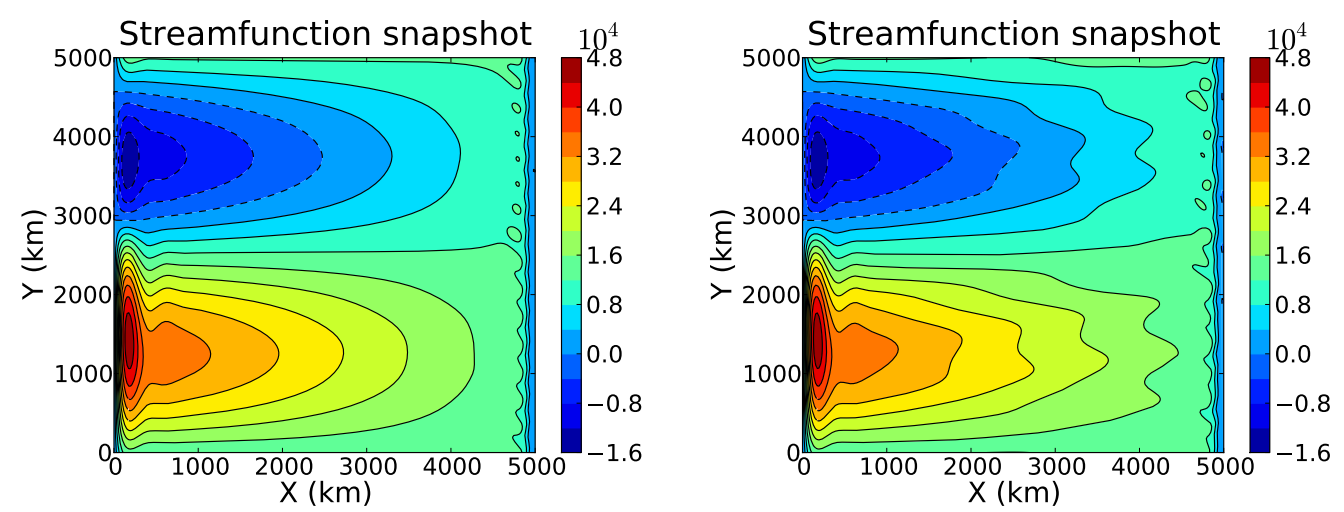

Figure 5-9: Streamfunction snapshots during PII (left) and PIII (right) in B25G100. The double-gyre structure is clearly shown in the ocean interior. The gyres are in a stable regime. The wavy structure in the interior during PIII is caused by the radiating instabilities.

to represent the EKE structures because this case has no meridional dependence. The EKE is trapped around the boundary region during PII (solid line) and spreads into the interior during PIII (dashed line) because of the long radiating mode, which is not a surprising result.

After applying the double-gyre forcing, the boundary EKE fields become nonuniform along the boundary (panel b1 for PII and b2 for PIII). The difference between b1 and the solid line in a1 is shown in c1. It represent the EKE differences between the cases with and without the large-scale flow influence during PII. During PII, the perturbations are mostly trapped, and the EKE increases over the southern domain after including the large-scale flow, but decreases over the northern domain (panel c1).

During PIII, when there are radiating modes, the EKE structure along the eastern boundary in b2 is different from the one in b1. The differences between b2 and b1 indicate the influence of the large-scale flow on the longwave mode. They are all positive, meaning EKE increases once the longwave modes are energetic. Panel a2 shows the meridional structure of $\mathrm{c} 2$ zonally averaged over a $300 \mathrm{~km}$-wide boundary box. The increase is meridionally non-uniform, showing a maximum at mid-latitude around $y=2500 \mathrm{~km}$ near the boundary. As a result, the meridional structure in a2 

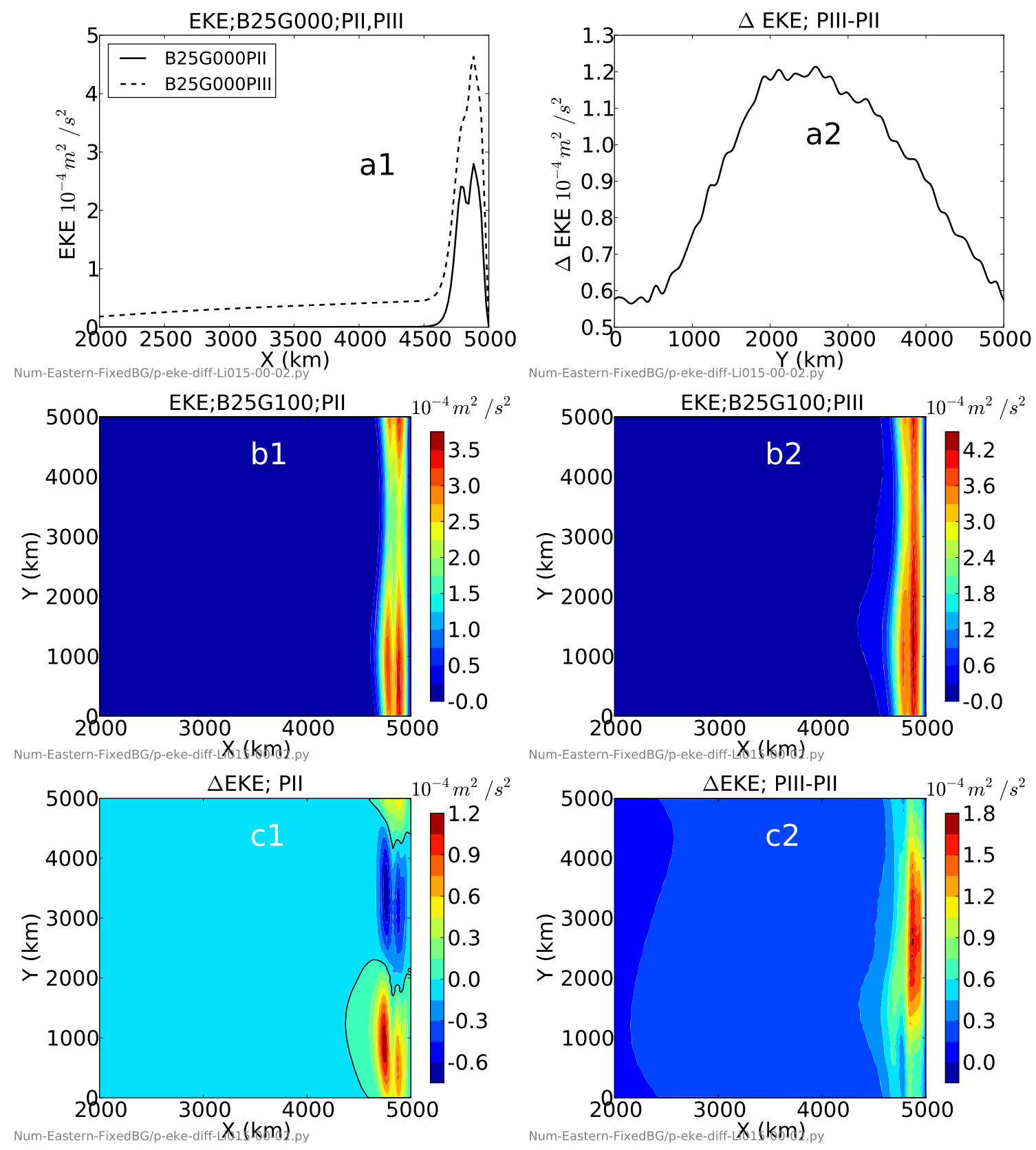

Figure 5-10: Panel a1 shows the zonal structure of the mean EKE in B25G000 during PII (solid line) and PIII (dashed line). Panel b1 and b2 show the mean EKE in BG25G100 during PII and PIII, respectively. Panel c1 shows the difference between EKE in B25G100 (b2) and EKE in B25G000 (solid line in a1). Panel c2 represents the difference between b2 and b1. The zonal average over a $300 \mathrm{~km}$-wide boundary region of $\mathrm{c} 2$ is shown in a2. 
is caused by the influence of the large-scale flow on the longwave mode or longwavemode-related mechanisms.

Panel c1 represents the large-scale flow influence on the trapped unstable mode, and $\mathrm{c} 2$ the influence on the long, linearly stable, radiating mode. It is obvious that the influences of the large-scale flow are different for different types of modes. The longwave modes are more energetic in the mid-latitude near the boundary, while the trapped unstable modes are more energetic over the southern boundary region. We next explain the mechanisms.

\subsection{Mechanisms}

The influence of the large-scale flow on the trapped modes is different than that on the long-radiating modes. We should realize that the influence on the trapped modes can be discussed without involving the longwave mode, but the influence on the longwave mode involves the shortwave mode because the shortwave mode energizes the longwave mode, as demonstrated in Chapter 3 (Figure 3-23). We first discuss the mechanism for the trapped modes during PII, then the one for the longwave modes during PIII.

\subsubsection{Phase - PII}

The large-scale flow influences the trapped perturbations along the boundary current, which leads to a non-uniform EKE distribution in the streamwise direction. Next we explain why there is such a meridional structure as shown in Figure 5-10.

In the linear stability study presented in Chapter 4, we used a prescribed largescale flow and a boundary current to study the behavior of imposed perturbations. Because the two elements in the mean state, the boundary current and the largescale flow, are fixed, they can not interact and modify each other. However, as in the nonlinear simulations, imposing a large-scale flow can change the boundary current structure. Although the large-scale flow is weak compared with the boundary current, 
a slight modification of the structure of the boundary current may lead to a change of the local stability, which can be reflected in the EKE field. The stability of the modified boundary current is our starting point to detect the causes of the EKE pattern shown in the panel $\mathrm{c} 1$ and $\mathrm{c} 2$ in Figure 5-10.

Figure 5-11 shows the time-mean of the meridional velocity field during PII in B25G100. We do observe structural changes in the meridional velocity field. The change could be caused by a combination of the gyre effect and the eddy feedback. Does the modification of the boundary current lead to a meridional non-uniformity

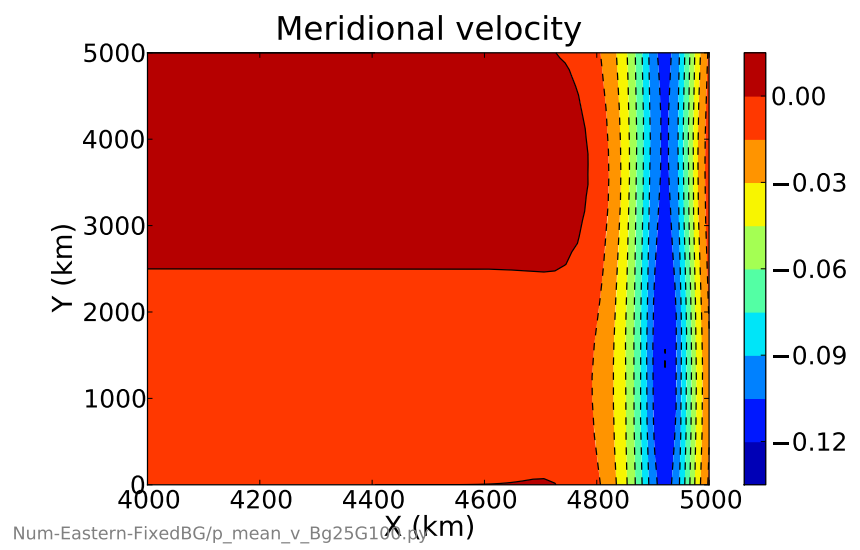

Figure 5-11: The time-mean meridional velocity during PII in B25G100. The meridional velocity is modified by the large-scale gyres.

of the localized instability?

As discussed in Chapter 4, the WKB approximation can be used to examine the local instability for a non-parallel boundary current, as long as the large-scale flow changes more slowly than the perturbation field does. In the two experiments discussed so far, the scales for the dominant perturbation and the large-scale flow are $333 \mathrm{~km}$ and $5000 \mathrm{~km}$. These values yield a ratio $333 / 5000=0.0666$, which is small, so that the local WKB approximation is a valid assumption for the two runs.

We conduct a temporal linear stability analysis based on the zonal profile of the mean meridional velocity, $\bar{v}\left(x, y_{s}\right)$, at a given latitude $y_{s}$. The temporal growth rates are solved by integrating the linear numerical model. The evolution of a specific mode 
at a given latitude $y_{s}$ is calculated by initializing the model with the corresponding sinusoidal mode. The growth rate for that mode is then computed by an exponential fit to ithe time series of the perturbation amplitude. We vary $y_{s}$ from 0 to $5000 \mathrm{~km}$ to compute the temporal growth rates as a function of wavelength and latitude.

Figure 5-12 shows the results. The color contours show that the maximum growth rate in B25G100 occurs around $y=1250 \mathrm{~km}$ with a wavelength about 312 to $333 \mathrm{~km}$, while the region between $y=3200 \mathrm{~km}$ and $4950 \mathrm{~km}$ becomes stable. The linear growth rates in B25G000, shown by the white contours, are uniform in $y$. This growth rate structure can be used to explain the $\Delta$ EKE structure in the panel c1 of Figure 5-10, which is as follows.

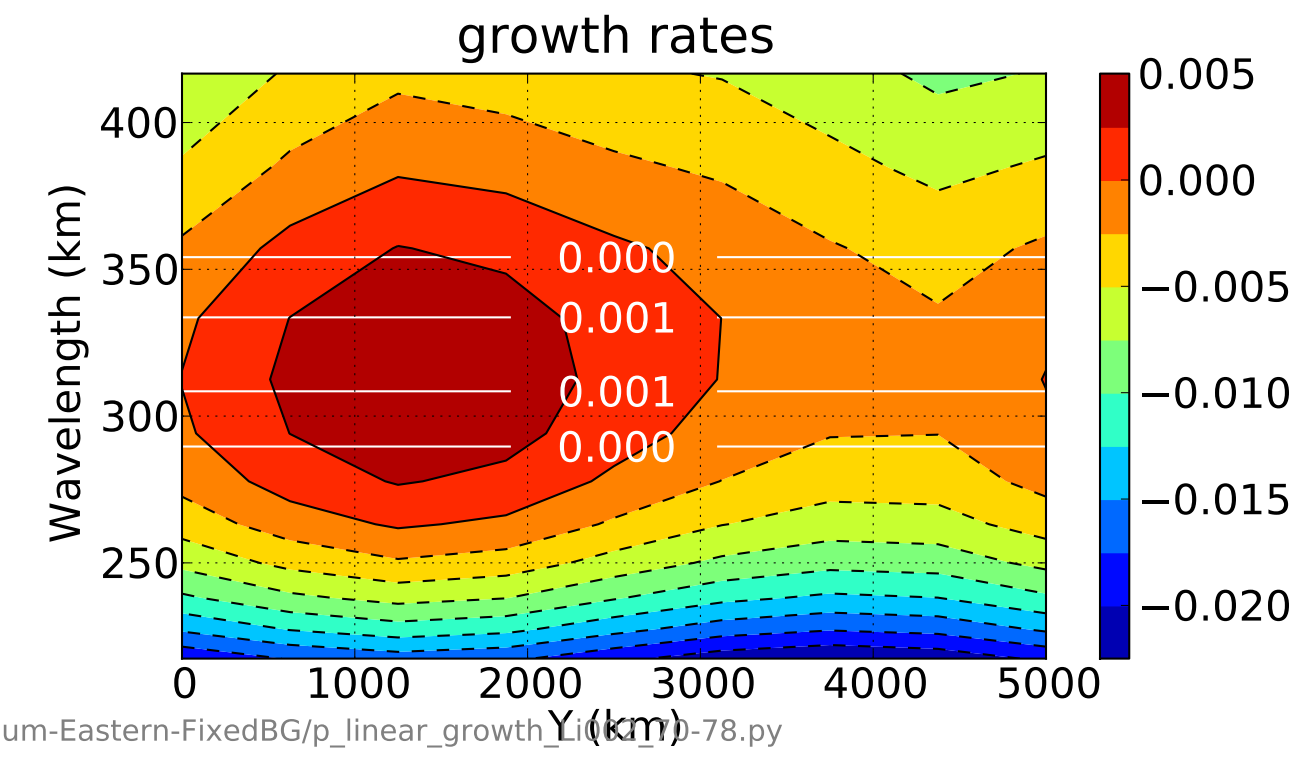

Figure 5-12: Local linear temporal growth rates as a function of wavelength and $y$. The maximum growth rate are around $y=1250 \mathrm{~km}$. The white contours indicate the growth rates based on the parallel boundary current. A superposition of the parallel boundary current and the large-scale flow (as done in the linear theory) gives the same growth rates contours because the large-scale flow has negligible contribution to the vorticity gradient, which determines the stability of a basic state.

We plot the maximum growth rate as a function of latitude in Figure 5-13 (the red curve), together with the one-dimensional meridional structure of the $\Delta \mathrm{EKE}$ between B25G100 and B25G000 during PII (shown in panel c1 of Figure 5-10). The 
unstable boundary current acts as an amplifier to energize the perturbations. In the case with a parallel boundary current, the eddy activity is uniform in $y$. One latitude can represent the whole system. However, in the case with a large-scale flow, the boundary current is modified and shows a localized maximum and minimum in terms of growth rate. Perturbations are energized over the more unstable region, but weakened over the stable region. The maximum eddy activity occurs downstream of the most unstable region. It is caused by the convective feature of the instabilities. The unstable perturbations grow and also propagate downstream (southward in this case). The downstream propagation leads to a spatial amplification, which is then reflected in the eddy activity field. The EKE starts to increase downstream at around

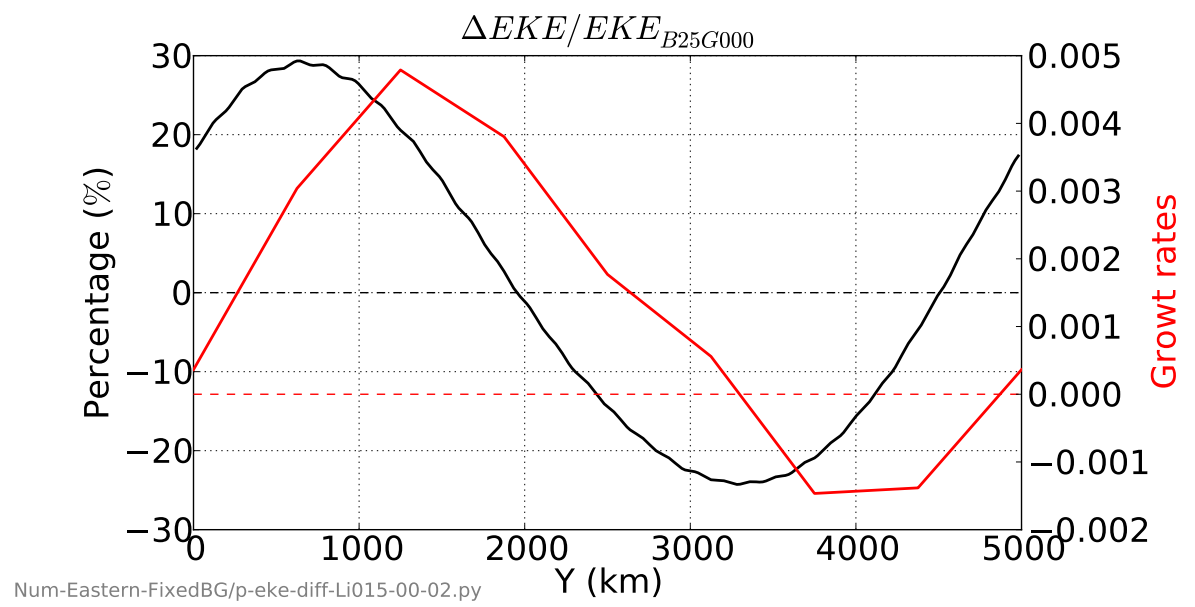

Figure 5-13: The black curve shows the meridional structure of the $\triangle \mathrm{EKE}$ averaged over a boundary region defined between $x=4700 \mathrm{~km}$ to $5000 \mathrm{~km}$. It is scaled by the mean EKE in B25G000 to indicate the significance of the EKE meridional deviations. For example, the EKE at $y=800 \mathrm{~km}$ increases about $30 \%$. The red curve shows the local maximum growth rate from the localized linear stability analysis. The horizontal dashed lines mark the zero values for each curve.

$y=3200 \mathrm{~km}$, where the local growth rate switches from negative to positive values. However, the EKE starts to decrease around $y=700 \mathrm{~km}$, where the local growth rates are still positive. This decrease is caused by the stabilizing effect of nonlinear and friction terms. The local energy input by the Reynolds stress becomes weak to the south of $y=700 \mathrm{~km}$, although positive, so that can not support the large amplitudes 
of the perturbations that propagate from the upstream more unstable regions.

Many studies regarding eddies in the mid-latitude atmospheric storm tracks also show similar downstream amplification. Chang and Orlanski (1993) studied the factors controlling the dynamics and maintenance of the eddy activity in a storm track using an idealized primitive equation model, and found that localized regions of enhanced baroclinicity do not necessarily lead to localization of eddy activity.

Pierrehumbert (1984) showed that the downstream development is related to the downstream advection of the instabilities. By linear studies, he concluded that in an infinite channel, only if the basic flow is absolutely unstable over some region, can localized modes occur over the region with locally enhanced baroclinicity; but no localized modes exist for a convectively-unstable basic flow. This phenomena is essentially embedded in the definition of convective instability. In periodic channels, global modes can be established after a few global traverse times even if the basic state is convectively unstable everywhere. This conclusion resembles our results. In both B25G000 and B25G100, the boundary current is convectively unstable everywhere, which is numerically tested. The global mode, represented by the EKE meridional structure, is established after many global traverse times. The energy is recycled across the north-south boundaries because of the periodic boundary conditions.

Up to now, we have explained the structure of the $\triangle \mathrm{EKE}$ by the structure of the local growth rates. Next we examine the eddy characteristics during PII. In the linear theory presented in the last chapter, perturbations are affected by the large-scale flow through the meridional velocity adjustment. With a same frequency, perturbations have smaller meridional wavelengths where the the large-scale flow is retrograde with respect to the boundary current, and have larger wavelengths where the large-scale flow accelerates the boundary jet (refer to Figure 4-26). In the following, the FFT analysis is used to diagnose the frequency and wavenumber spectra of the eddy field in the nonlinear simulations.

The frequency spectra can be computed based on the time series for a fixed station. We compute the frequency spectra for stations inside the boundary current region 
$(x=4863 \mathrm{~km}$ in this case) at each latitude. Figure 5 -14 shows the frequency spectra for B25G000 and B25G100 as a function of frequency and $y$. As expected, there is only one dominant mode. The frequency at a fixed station is not changed by the large-scale flow. The weakening effect over the northern domain can be seen in the right panel. This is the power density expression of the weakening feature in the EKE field (refer to panel b1 in Figure 5-10).
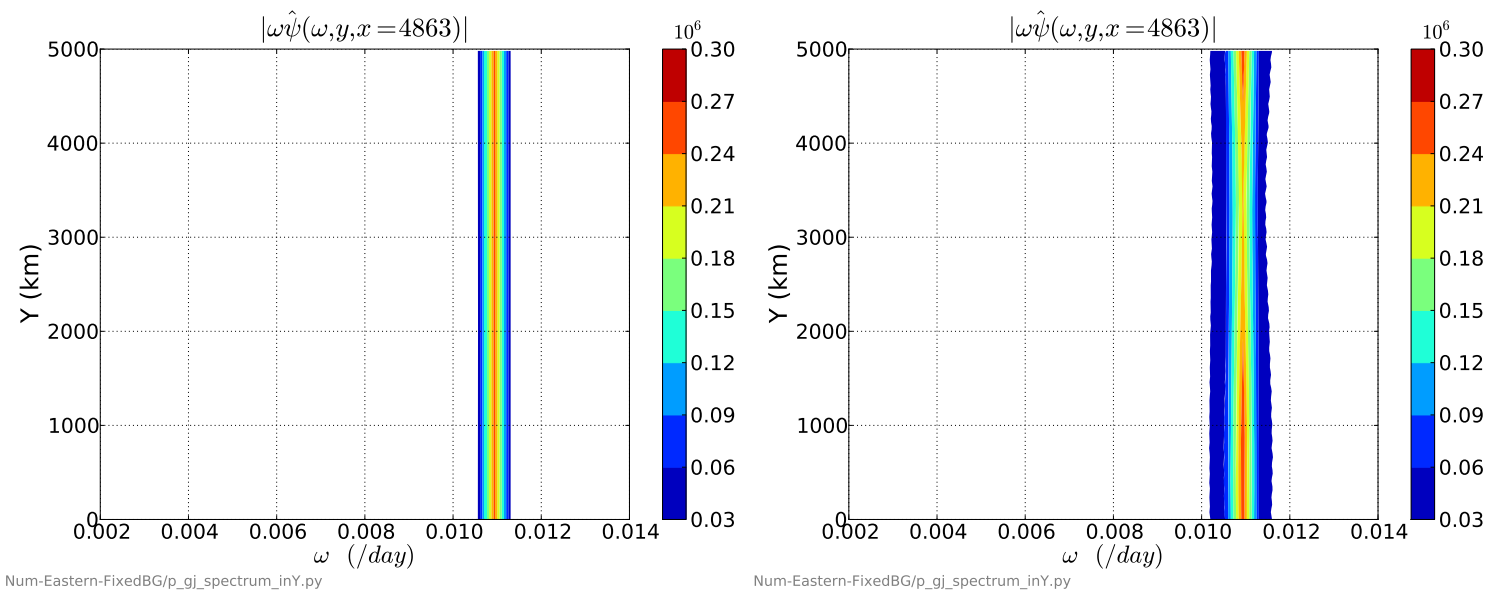

Figure 5-14: The frequency spectra as a function of wavenumber and $y$. The left panel is for B25G000, and the right for B25G100. The large-scale flow does not change the frequency of the unstable perturbations which is evident from the straight contour lines in the right panel, but changes the intensity of the perturbations at different latitudes. The weakening effect over the northern domain represents the same weakening feature in the $\triangle$ EKE field. Values less than 0.03 are not plotted.

According to the linear theory, a perturbation field with the same frequency has a larger wavelength over the southern domain, and has a smaller wavelength over the northern domain, because of the effect of the meridional velocity adjustment. The frequency of the perturbations is demonstrated to be unchanged. The wavenumber spectra are expected to show a meridional non-uniformity.

Once the boundary current possesses a meridional structure, diagnosing meridional wavenumber spectra becomes less obvious. With a meridionally varying largescale flow, the meridional normal modes are no longer solutions, and the basin-wide FFT analysis becomes less meaningful for studying eddy behaviors. Here instead of 


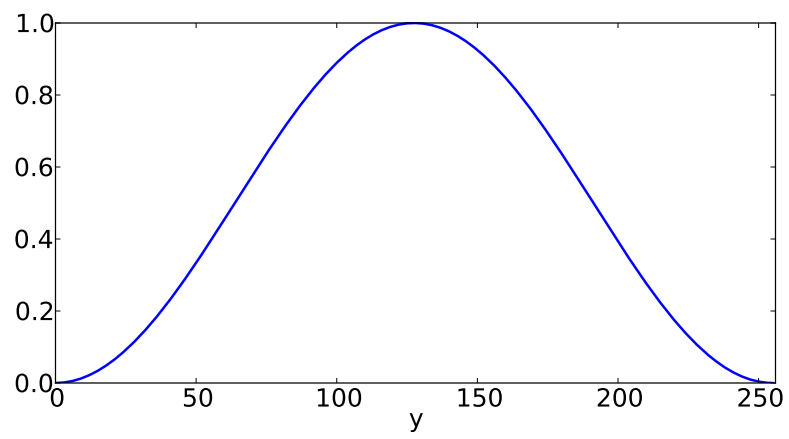

Figure 5-15: An example of the Hanning function.

conducting an FFT over the whole domain, we apply the essence of WKB approximation and conduct the FFT over a perturbation field that is spatially window-tapered to examine the local wavenumber spectra. The localized window filter diminishes the contribution of the signal outside of the focus area and reveals the local eddy-scale behaviors.

The Hanning function

$$
\begin{aligned}
W\left(y ; y_{s}, L_{W}\right) & =\sin ^{2}\left[\pi\left(\frac{L_{W} / 2+y_{s}-y}{L_{W}}\right)\right] ;\left|y-y_{s}\right| \leq L_{W} / 2 \\
& =0 ;\left|y-y_{s}\right|>L_{W} / 2
\end{aligned}
$$

is used as the window function, where $L_{W}$ is the window size, and $y_{s}$ is the fixed station that the spectra is computed for. Figure 5-15 shows an example of the Hanning function.

We move the center of the window function from $0 \mathrm{~km}$ to $5000 \mathrm{~km}$ to calculate the local wavenumber spectra for every meridional station $y_{s}$. Since the meridional boundary condition is periodic, we join the northern/southern half to the southern/northern boundary to expand the field, so that there are no missing data when the center of the window function is close to the meridional boundaries. The FFT analysis is then applied to the tapered field to calculate the local wavenumber spectra 
at $y=y_{s}$. The process is formulated by

$$
\hat{\psi}\left(l ; x_{s}, y_{s}, t_{s}\right)=\mathfrak{f}\left[\psi\left(y ; x_{s}, t_{s}\right) W\left(y ; y_{s}, L_{W}\right)\right]
$$

where $W$ is the window function, $x_{s}$ and $t_{s}$ represent the zonal location and the time of the sample, and $\mathfrak{f}$ represents the Fourier transform.

An ensemble mean is taken over several time steps and zonal locations to average out the noise. When we interpret the result, we should keep in mind that the Hanning window reduces the spectral resolution, and widens the local lobe which can be seen in the following calculations. Different window-size will give different quantitative results, but do not change the qualitative conclusion. Several values are tested to give the same feature. We show the one with $L_{W}=5000 \mathrm{~km}$ here. All the calculations are carried within a boundary region between $x=4900 \mathrm{~km}$ and $x=5000 \mathrm{~km}$.

Figure 5-16 shows the wavenumber spectra as a function of meridional wavenumber and $y$ for the case B25G000 and B25G100. In B25G000 (left panel) without the large-
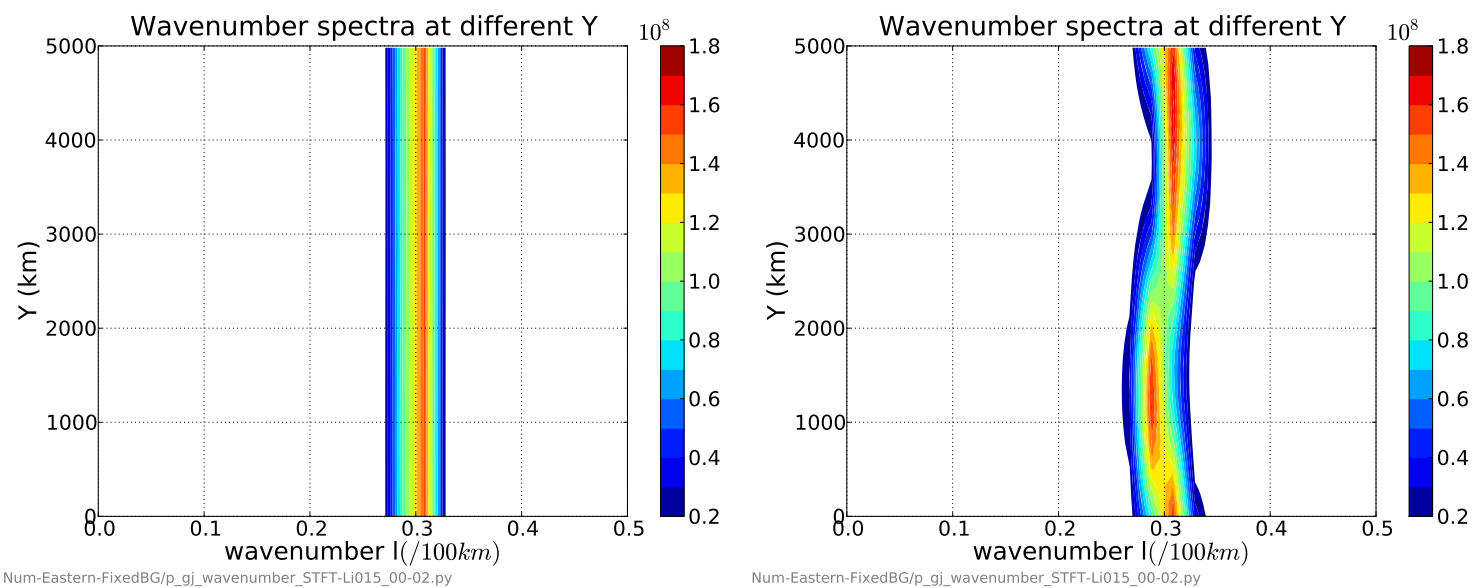

Figure 5-16: The wavenumber spectra as a function of meridional wavenumber and $y$ for the case B25G000 (left) and B25G100 (right). The Hanning window is applied in the FFT calculations. Values less than 0.2 are not plotted.

scale flow influence, the eddy field has uniform meridional wavenumber spectra. The dominant mode has a wavelength $333 \mathrm{~km}$, which is also shown in Figure 5-5. The case B25G100 is shown in the right panel of Figure 5-16. It is clear that the wavenumber is 
higher over the northern domain and longer in the southern domain, which is predicted by the linear analysis. Over the northern domain, where the gyre flows against the boundary current, perturbations are squeezed and have larger wavenumbers, while the opposite is true over the southern domain. We will see in the next section that the wavenumber shift affects those long-radiating modes.

\subsubsection{Phase - PIII}

During PIII, the long-radiating modes are energized by the nonlinear triad-resonance. The energy distribution of the longwave modes is different with that of the unstable trapped modes under the influence of the large-scale flow. Figure 5-10 shows that the EKE of PIII is larger than that of PII along the boundary with a maximum located at the middle of domain around $y=2500 \mathrm{~km}$. The location of the maximum during PIII is different than that of the PII.

As discussed in Chapter 3, the longwave mode is generated by the nonlinear triadresonance. The growth rate of the long-radiating mode is a function of the amplitude of the most unstable trapped-mode and the phase mismatch among a triad of waves. On the one hand, the amplitude of the longwave mode varies with the amplitude of the trapped mode, which is essentially controlled by the forcing strength. On the other hand, when the phase mismatch becomes large, the triad-resonance becomes weak, so that the longwave mode becomes less energetic.

The longwave modes have wavelengths that are more than 600km (Figure 5-5), which is within the wavelength region of negative growth rates. The longwave mode can not sustain itself. Its existence has to rely on the nonlinear energy transfer. The structure of the EKE of the longwave mode does not follow that of the trapped mode, which is further related to the local boundary-current stability as shown in Figure 5-12. The possible explanation is related to the modified local efficiency of nonlinear energy transfer because of the changes in the phase mismatch.

We conduct the same frequency and wavenumber spectral analyses as shown in Figure 5-14 and 5-16 to gain better idea of the characteristics of individual modes. 
Figure 5-17 is the same as Figure 5-14 but for the PIII. The left panel shows the case B25G000 without gyres. The low-frequency mode (equivalently the longradiating mode) becomes significant. As there is no gyre influence, the frequency spectrum is uniform in $y$.
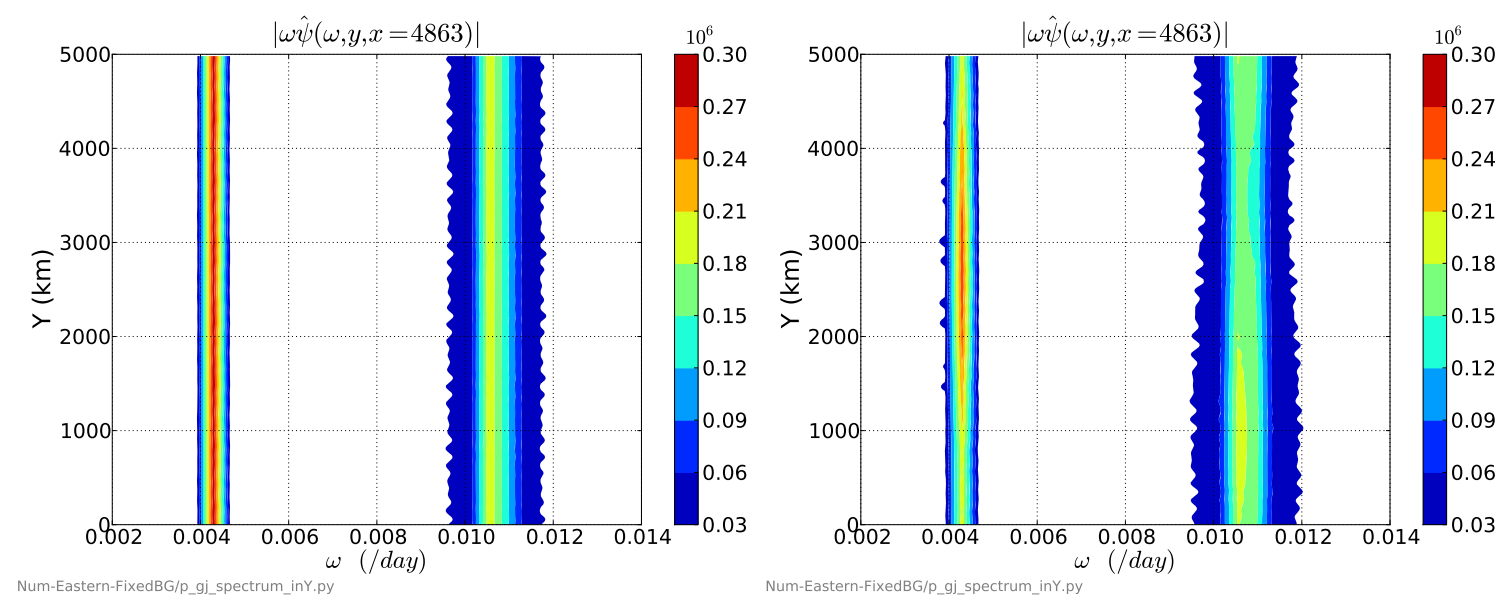

Figure 5-17: The same figure as Figure 5-14 but for PIII. The left panel represents the PIII in case B25G000, and the right panel represents the PIII in case B25G100. Values less than 0.03 are not plotted.

The right panel is for the PIII in the case B25G100. We notice that the unstable trapped mode in PIII has similar structure to the one in PII, which is weaker over the northern domain but stronger over the southern domain. This feature means that the emergence of the long-radiating mode does not change the characteristics of the trapped mode.

However, the spectrum of the longwave mode exhibits a different meridional structure compared with that of the trapped mode. The bigger amplitude occurs in the middle of the domain instead of coinciding with the structure of the trapped mode. The low-frequency mode also has longer wavelength, which is explicitly shown in Chapter 3, and can also be confirmed here as the longwave mode and low-frequency mode both appear in the spectra plot during PIII, but not during PII. The meridional structure of the low-frequency mode confirms that the longwave modes are the cause for the $\triangle \mathrm{EKE}$ maximizing in the middle domain shown in Figure 5-10. 
Figure 5-18 shows the wavenumber spectra for PIII in both B25G000 (left panel) and B25G100 (right panel). The meridional structure of the spectra in the shorttrapped mode is unchanged from PII to PIII, but the extra longwave modes occur in PIII. These modes have constant power density without the gyre influence (left) but show a maximum in the middle domain after the gyres are included in the system (right panel). This maximum corresponds to the same maximum of the longwave modes in the frequency spectra. Both of them are spectral expressions of the EKE structure of the longwave modes.
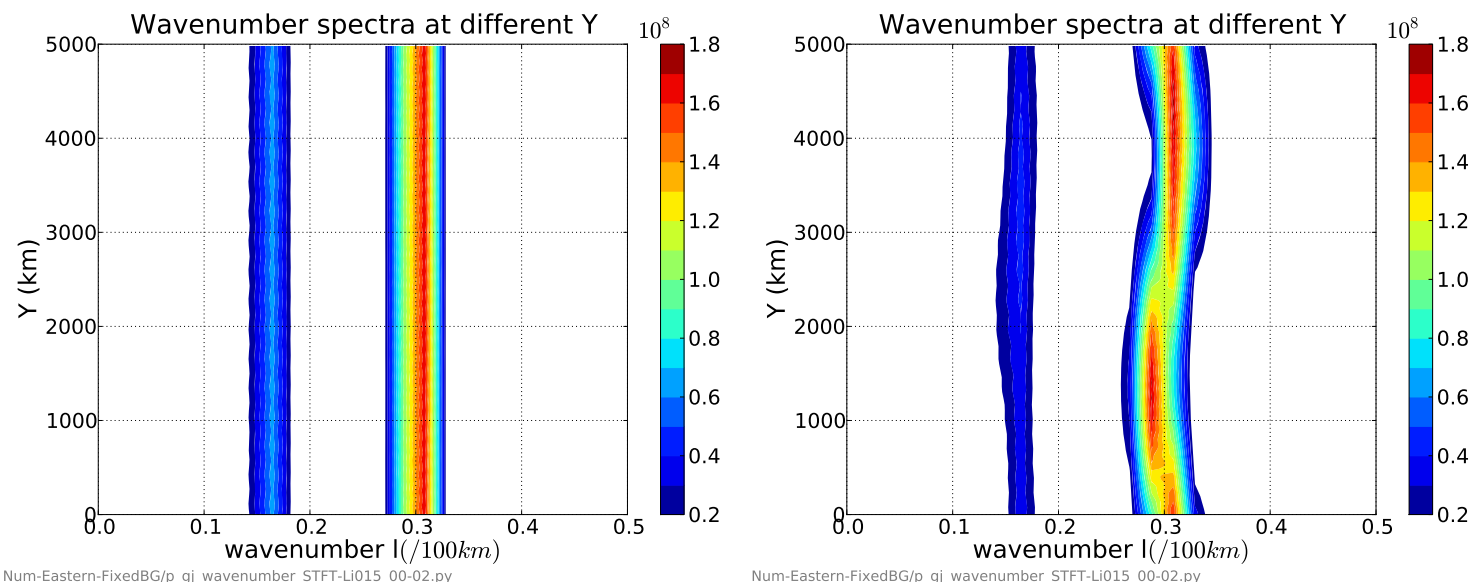

Figure 5-18: The same as Figure 5-16 but for PIII. The long-radiating modes are generated during PIII. Their spectra are uniform in $y$ in the case B25G000 (left), but show a local maximum in the middle domain in the case B25G100 (right).

The evidence in the wavenumber and frequency spectra demonstrates that the structure of the $\triangle \mathrm{EKE}$ in Figure $5-10$ is induced by the radiating mode with long wavelengths and low frequencies. The significance of the longwave mode may be questionable because of the small amplitude in this case. However, this longwave mode becomes more energetic following an increase of nonlinearity. Figure 5-19 shows the EKE distribution in B30G100. The radiating mode becomes energetic, and the total EKE field shows a maximum over the middle domain, instead of over the southern domain in B25G100 (Figure 5-10).

The different characteristics of the EKE between the radiating mode and the 


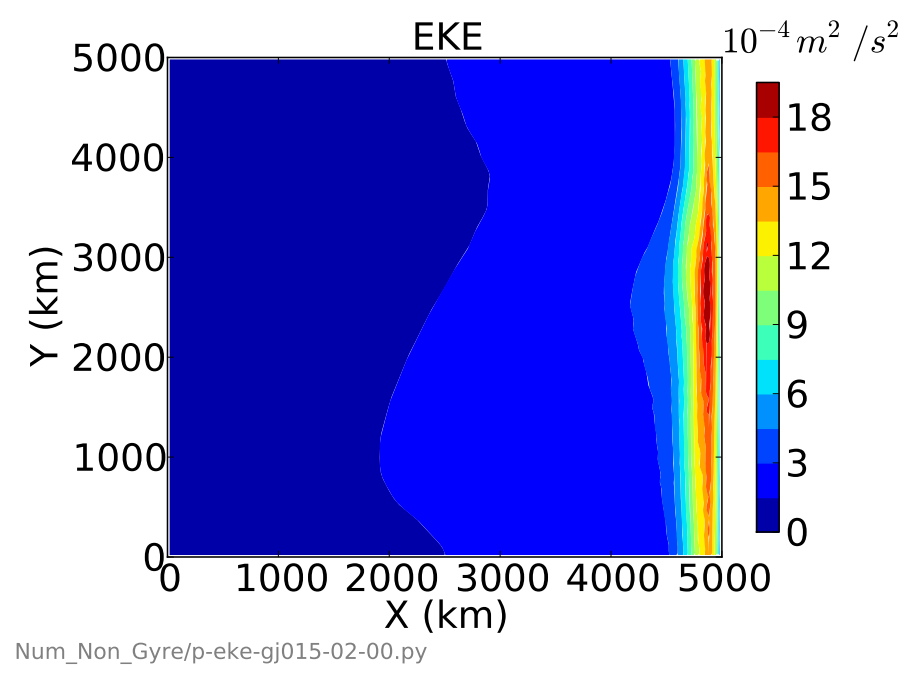

Figure 5-19: Total EKE in B30G1000. Following the increase of nonlinearity, the longwave mode becomes energetically important.

trapped mode are rather puzzling. The maximum EKE occurs over the southern domain along the boundary for the trapped mode, while over the mid-latitude along the boundary for the radiating mode. As the long and low-frequency mode is solely generated by the trapped mode through the nonlinear triad-interaction, then why does not the strongest longwave mode occur at the location of the strongest trapped mode, but rather upstream?

We propose a tentative explanation here based on the results of previous chapters. In the nonlinear study of a parallel boundary current presented in Chapter 3, we see that the subharmonic mode (the long-radiating mode) is powered by its superharmonic mode, which is trapped. The growth rate of the subharmonic mode depends not only on the amplitude of the superharmonic mode, but also on the phase mismatch. The trapped modes and the longwave modes do not share the same structure, showing maxima and minima at different locations. As a result, the phase relation becomes a possible explanation. Through a local FFT analysis, we show that the structure of a traveling wave is modified along the boundary as a consequence of the meridioinal velocity adjustment. Perturbations are stretched or squeezed when the large-scale flow is strongly against or with the boundary current. The phase mis- 
match is a minimum near the stagnation points around $y=0$ and $y=2500 \mathrm{~km}$. The local growth rates, which represent the efficiency of the Reynolds energy-input into the unstable mode, are larger at $y=2500 \mathrm{~km}$ than at $y=0$ (refer to Figure 5 -12). The overall effect leads to the concentrated EKE maximum in the longwave modes residing around the mid-latitude at $y=2500 \mathrm{~km}$ near the boundary.

Finally, we show the meridional structures of EKE in other experiments that are listed in Table 5.1. The nonlinear energy transfer is a slow process for weak nonlinearity, so the longwave mode takes a long time to develop, which leads to a clearly defined period, PII, with only trapped modes (Figure 5-8).

The left panel in Figure 5-20 shows the ratio of meridional deviation of EKE to the mean value during PII in the weakly nonlinear cases with different gyre strengths. We see that the basic structures are in agreement. The meridional non-homogeneity increases following the increase of the gyre strength. The right panel shows the same quantity but for cases with a stronger $F_{b}$. In those cases, the longwave modes and the trapped unstable modes are generated almost simultaneously, leaving phase PII barely definable. The quantity shown in the right panel is calculated during PIII when the longwave modes are well developed. We see that the total EKE field shows a maximum in the mid-latitude near the boundary. An energy decomposition (figure is not shown here) indicates that the longwave modes become energetic, and its contribution surpasses that of the trapped modes. The increase in the gyre strength leads to the enhanced meridional non-homogeneity.

In Figure 5-20, we only show cases with two different forcing strengths. The energy of the longwave mode gradually increases following the increase of the nonlinearity. The final EKE curve is a combination of the contributions of the unstable mode and the longwave mode. Consequently, the significance of the longwave mode determines how much the final EKE curve drifts away from the curves in the left panel. 

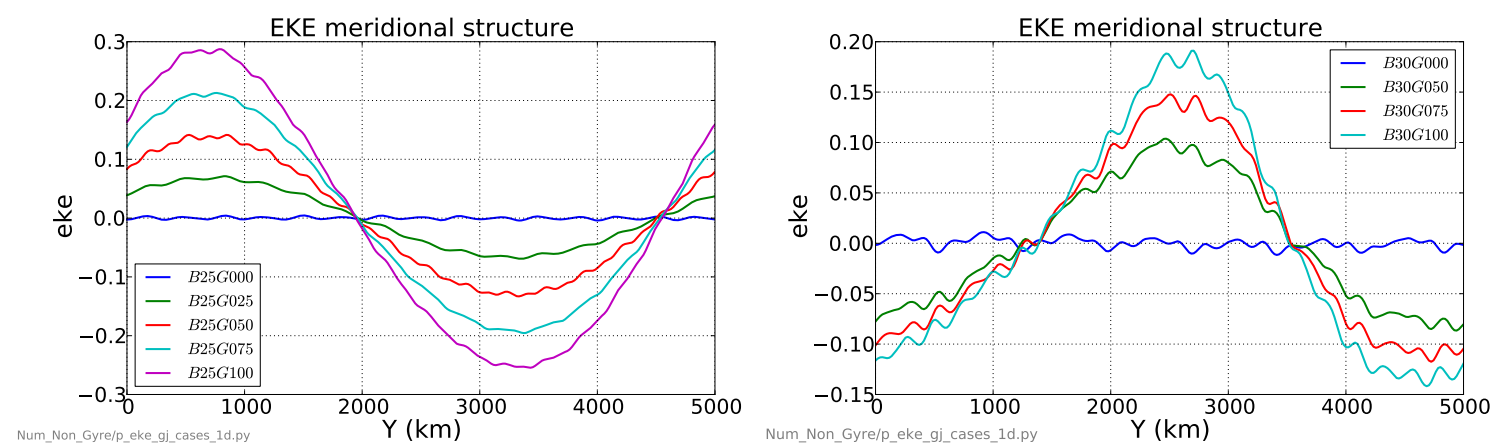

Figure 5-20: The left panel shows the meridional deviation of the boundary-regionaveraged EKE scaled by its mean value during PII in cases with weak nonlinearities. The right panel shows those in the cases with stronger $F_{b}$ (equivalently stronger nonlinearities). With the contribution of the longwave modes surpassing that of the trapped modes, the total EKE shows a maximum in the middle of the domain.

\subsection{Conclusion and discussion}

In this chapter, we use the eddy kinetic energy as an index to study the influence of the large-scale flow on the stability of an eastern boundary current, and find that the influence of the large-scale flow on trapped unstable modes is different than that on long, radiating modes.

The large-scale flow can affect the trapped unstable mode by modifying the mean structure of the boundary current to induce a meridional non-homogeneity in the local stability, which alters the linear assumption when the boundary current is assumed to be fixed and can not be changed by the large-scale flow. With the parameters used in this chapter, the modified boundary current becomes more unstable over the southern domain but more stable over the northern domain (Figure 5-12). The localized maximum and minimum in growth rates lead to non-homogeneity in EKE field. The maximum EKE occurs downstream of the most unstable region because of the instabilities' convective characteristics.

The large-scale flow also induces an influence on the structures of the trapped unstable perturbations. It affects the trapped modes through the meridional velocity adjustment as discussed in the linear theory in Chapter 4. The unstable mode becomes shorter over the northern domain since it is compressed by the gyre, and 
longer over the southern domain since it is stretched. Traveling from north to south, the shortened waves are elongated again.

Furthermore, the alteration of the trapped modes by the large-scale flow leads to an indirect influence of the large-scale flow on the long-radiating modes. The longwave modes that appear in the nonlinear simulations are not self-sustained, but rather rely on the nonlinear energy transfer from the trapped modes through nonlinear triad-resonance. The efficiency of the nonlinear energy transfer depends on the amplitude of the trapped modes and also the phase relation of the wave-triad. The meridional velocity adjustment effect of the large-scale flow leads to shortened and elongated perturbations over the upstream and downstream regions, where the phase relation is less satisfied and the nonlinear triad-resonance diminishes. The minimum mismatch occurs around the stagnation points at $y=0 \mathrm{~km}$ and $y=2500 \mathrm{~km}$ near the boundary according to the linear theory. However, the positive linear growth rates, which represent the efficiency of the energy transfer from the mean to the unstable modes, diminish both in amplitude and wavenumber range at the stagnation point $y=0 \mathrm{~km}$ (refer to Figure 5-12), but increases at the stagnation point $y=2500 \mathrm{~km}$. The discrepancies between these two points are due to the zonal velocities of the largescale circulation. The eastward flow at $y=2500 \mathrm{~km}$ compresses the boundary current to induce larger growth rates. The opposite is true for $y=0 \mathrm{~km}$. The combined effect leads to the EKE maximum of the longwave modes residing at the domain middle around $y=2500 \mathrm{~km}$. The long radiating mode becomes more energetic following the increase of nonlinearity. The EKE of the long radiating mode can surpass the trapped modes and dominate in the total EKE field.

The different influences of the large-scale flow on the trapped modes and longradiating modes are further tested using cases with different parameters. Stronger large-scale flows increase the meridional non-homogeneity in the eddy field. The deviation can be as large as 40 percent of increase or decrease.

Some of the linear results in Chapter 4 are evident in the nonlinear simulations. The meridional velocity adjustment effect is clear in the trapped perturbation field. 
Some of those linear results are not valid in the nonlinear simulations. In the linear theory, the boundary current is prescribed and can not be freely changed by the large-scale flow. The linear results mostly concern the influence on perturbation fields (unstable modes in our discussion), rather than the influence of the large-scale flow on the mean boundary current, which in turn is important in generating the meridional EKE non-homogeneity of perturbations as demonstrated in this chapter. The global mode that occurs around the northern domain in the linear theory will not occur in the nonlinear simulations because the absolute instability never occurs over the northern domain where the boundary current is modified to be stable. Finally, there are phenomena in nonlinear simulations that are absent in, but related to, the linear theory. The activities of long-radiating modes that are generated by nonlinear triad-resonance are related to the phase mismatch of the trapped unstable modes, but it is a nonlinear phenomenon that is only observable in nonlinear simulations.

Although we try to understand the parameter dependence by running more experiments, we realize that the parameter range is quite wide, and a big part of parameter space has not been explored in this study. For example, we can use different functions other than the Bickley jet profile to test the dependence of the meridional nonhomogeneity on the boundary current structures. Other nondimensional numbers also add more possibilities.

The merit of this study is to demonstrate the importance of the large-scale flow in modulating the boundary current instabilities and to identify mechanisms based on wave kinematics which could be used in explaining similar phenomena. We do not expect the meridional EKE structure evident in this study to be universal. Certainly, different boundary current structures yield different sets of growth rates and phase/group velocities for unstable modes. However, the same machinery used here can be applied in other situations to accommodate the explanation of different phenomena.

Any parameter that changes the convective/absolute features of the instabilities can significantly modify the EKE structure. The EKE structure in this chapter can 
be regarded as the 'global mode'. This global mode is less physically relevant because the boundary current is convectively unstable everywhere, and that global mode will disappear without the periodic boundary condition (or energy recycling). The absolute instability does not occur in the nonlinear simulations because the boundary current is nonlinearly modified. The absolutely unstable region in the linear theory becomes stable in the nonlinear simulations. A true global mode can form once absolute instabilities emerge. That global mode is more easily affected by the largescale flow because its propagation speed is infinitesimal (thinking about a stationary wave packet) and any small additional along-boundary velocity from a large-scale flow can shift the global mode tremendously. Furthermore, the structure of the basic state used here can be arbitrary. One can always calculate the forcing field from any basic state velocity field according to the relationship $\mathcal{F}=\beta \bar{v}-\partial_{x} A_{h} \partial_{x} \bar{v}$. It will be interesting to consider other velocity profiles.

Rossby wave propagation is often constrained by geostrophic contours. The barotropic model used here has no bottom topography or isopycnal slope. The largescale flow is also weak so that the geostrophic contours are simply $\beta y$, and we do not observe significantly curved wave rays. However, the instabilities radiated from an EBC are expected to be modified by a baroclinic interior gyre which associates with sloped isopycnals. They will show different behaviors when they propagate westward depending on their baroclinic structures. Those are topics and challenges for future study. 
THIS PAGE INTENTIONALLY LEFT BLANK 


\section{Chapter 6}

\section{Conclusion}

Unstable eastern oceanic boundary currents generate mesoscale/submesoscale eddies that are visible in satellite images. It has been proposed and observed that these small scale features can propagate westward to become an energy source for the ocean interior. However, the dynamical mechanisms by which the instabilities radiate and penetrate into the interior remain largely unexplored. Furthermore, the large-scale flow influence on the stability of an eastern boundary current has not been previously studied. Here we explore the instability of an eastern boundary current (EBC) with and without a large-scale flow in an idealized framework represented by barotropic quasi-geostrophic dynamics. The barotropic quasi-geostrophic model is used as a starting point. This simplicity helps to focus on a limited number of dynamical factors and to identify mechanisms that can be used for future studies.

\subsection{Instability of an eastern boundary current}

The observed eddy variability in the ocean interior has motivated many studies to investigate the importance of the energy radiation originated by swift unstable currents. Early studies focused on the energy radiation from a zonal current or a zonally moving boundary mimicking the Gulf Stream (Flierl and Kamenkovich, 1975; Pedlosky, 1977; Harrison and Robinson, 1979; Malanotte-Rizzoli et al., 1987). It was 
found that energy mostly cannot radiate from eastward zonal currents (Talley, 1983). Later studies show that radiating instabilities occur more easily for a non-zonal current (Kamenkovich and Pedlosky, 1996, 1998a,b). It was also shown that an unstable meridional western boundary current (WBC) can generate eastward radiating instabilities even in the presence of friction (Fantini and Tung, 1987). Recently, Hristova et al. (2008) showed that an unstable EBC supports a greater number of radiating unstable modes over a larger range of meridional wavenumbers than an unstable WBC. However, the study investigated in linear regime only with a highly idealized boundary current represented by a piecewise constant velocity profile. Here we extend the study of the instability of a meridional boundary current by considering a continuous velocity profile in both linear and nonlinear regimes.

In the linear theory, a shortwave cutoff appears in the growth rate curve for the instability of a continuous velocity profile. The cutoff is absent for the piecewise velocity profile used in the previous studies. Piecewise profiles have a velocity jump at the transition point. Perturbations, no matter how small they are in the horizontal scale, can "feel" the infinite velocity shear at that point, and hence can always grow. However, small perturbations can become stable in a continuous current because they can only "feel" a partial profile, which may have a stable structure. The shortwave cutoff defines the lower scale limit for perturbations to be unstable.

The shortwave cutoff of an EBC shifts to longer wavelengths following the increase of beta, meaning that shortwaves tend to be stabilized by the beta effect. In a parallel study, we find the shortwave cutoff of a WBC shifts to the shortwave end, now implying that the beta effect destabilizes meridional shortwave modes. The growth rate of an unstable mode in a barotropic jet relates to the energy input by the Reynolds stress, $-u v V_{x}$ for a meridional current. The phase tilt of the streamfunction, measured by $u v$, largely determines the growth rate for a particular mode. When $\beta$ increases, the perturbation streamlines of unstable modes tilt more against the mean shear in the WBC, but less so in the EBC, resulting in a more stable EBC and a more unstable WBC. When beta increases, the EBC also supports less unstable 
modes, while the unstable wavenumber range systematically shifts to the shortwave end for the WBC. The WBC is further tested in a larger beta space. The maximum growth rate increases without any limit when beta increases. This asymmetry in growth rates between the EBC and WBC has not been shown in previous studies.

The beta effect increases the meridional phase speed of the perturbation in the WBC but reduces the phase speed in the EBC. The phase speed increase in a WBC is also observed in Fantini and Tung (1987) and Hristova et al. (2008), in which a piecewise constant velocity profile is used. The mechanisms, however, are unknown. The different responses of the phase speed (correspondingly frequency) to the beta increase bring interesting asymmetries between the EBC and the WBC instabilities. Given the same meridional wavenumber, the unstable mode has smaller zonal wavelength in the EBC than in the WBC; while given the same frequency, the unstable mode has a larger zonal wavelength in the $\mathrm{EBC}$ than in the $\mathrm{WBC}$, consistently with the result of Hristova et al. (2008). However, this study finds that the WBC supports more unstable modes than the EBC, which contradicts Hristova et al. (2008). A reproduction of their results would be helpful for the comparison, but will await a future investigation.

Regarding the instability radiation, we found that long meridional waves are more able to radiate, but have different zonal wavelengths depending on the radiating direction, consistent with previous studies (Kamenkovich and Pedlosky, 1996; Fantini and Tung, 1987; Hristova et al., 2008). For an isolated meridional jet, zonal shortwave modes radiate eastward, but zonal longwave modes radiate westward. This result is also consistent with that of Kamenkovich and Pedlosky (1996). The cross-stream structure in their study resembles our zonal structure. Considering an EBC, only a few longwave modes can radiate.

Energetically, the few meridional long radiating modes may not be significant for the interior because of their small growth rates. The stability analysis of an EBC is therefore extended to the nonlinear regime. It is found that the linearly decaying and radiating modes can resonate with an unstable trapped mode to become nonlinearly 
unstable and then play important roles in the energy budgets of an EBC.

In our experiments, the nonlinear processes relate to the subharmonic instability, caused by the nonlinear resonance between two harmonics. Even if there is only one unstable mode, the stable longwave mode can still grow through this process. The nonlinear generation of the longwave mode changes the energy budget of an EBC system. The subharmonic mode is a radiating mode with a long meridional wavelength. Before it becomes energetically significant, the energy budget of an EBC is balanced locally. The unstable modes draw energy from the mean boundary current through the Reynolds stress, and drain energy locally through frictional energy dissipation. The subharmonic mode radiates a significant amount of eddy kinetic energy away from the boundary region after reaching a large amplitude. The instability radiation becomes an energy sink. In the experiment presented in Chapter 3, 25 percent of the total energy-input into the eddy field is radiated away by the long radiating mode.

It is noteworthy that the longwave mode is a linearly stable mode for the boundary current, but can still draw energy from the mean after reaching a finite amplitude. This means that the mode is modified by the nonlinear processes, having a structure that can draw energy from the mean. This mode is partially self-sustained, but vanishes without the nonlinear energy transfer, because the total energy sink through the radiation and frictional dissipation is greater than its energy gain from the mean current.

This nonlinear instability radiation may explain the observed zonal jets in the ocean interior. Maximenko et al. (2005) discovered from satellite altimeter data the ubiquity of zonal jets in the ocean. This phenomenon has provoked an ongoing debate about their origins. Hristova et al. (2008) hypothesize that the interior zonal jets are due to the propagation of the radiating instabilities. The radiating modes have zonal wavelengths much larger than the meridional wavelengths, so that they show a zonal-jet structure in the interior. This study shows that the nonlinear radiating instabilities can produce zonal jets in the interior with a magnitude that is comparable to the observed values. The interior velocity of the radiating mode in this study is 
about $5 \mathrm{~cm} / \mathrm{s}$, which is comparable to $6.9 \mathrm{~cm} / \mathrm{s}$ found in Maximenko et al. (2005). The meridional wavelength of the radiating mode is approximately $700 \mathrm{~km}$, about twice the observed value $(300 \mathrm{~km})$. Note that the wavelength of the radiating mode is related to the boundary current width, about $80 \mathrm{~km}$ in our study, which is about twice the width of most of the EBC systems. A $40 \mathrm{~km}$-wide boundary current may produce zonal jets with a meridional wavelength around $300 \mathrm{~km}$. The main point is that one very idealized EBC can produce nearly zonal interior flows roughly consistent with observations.

However, the radiating instabilities are transient waves with nonzero meridional phase speeds, while the observed zonal jets are rather stationary. Can these two phenomena be associated with each other? Certainly the model of a parallel EBC alone used in this study can not explain the stationary zonal jets. Hristova et al. (2008) also found nonzero meridional phase speeds for their radiating modes. This situation may change after considering the irregularity of the coastal bottom topography or coastlines. The irregularity of the coastline and topography is very important for anchoring coastal filaments and for enhancing the growth of meanders and eddies (Kelly, 1985; Batteen, 1997; Brink and Cowles, 1991). One can expect that the longtime average of radiating instabilities in the interior will have a finite amplitude because of the spatially irregular but stationary continental coasts. The time-mean field can then show traces of the long radiating modes emitted from the EBCs. These arguments cannot directly prove the relationship between the formation of the zonal jets and the radiating instabilities, but provide a plausible mechanism. This hypothesis, originally raised in the linear analysis in Hristova et al. (2008), is strengthened by the linear and nonlinear studies in this thesis.

The experiments in this study are in a weakly nonlinear regime, and the subharmonic instability is only one type of the triad-resonance. We suggest that the strong nonlinearity and broad triad-interactions could make the nonlinear energy transfer even more important in the instability/energy radiation. Then an important question to ask is what is the energy budget for a fully developed eastern boundary system? 
This question can not be answered by the simple model here used, and the inclusion of complex dynamics, such as the baroclinicity and sloping bottom, are necessary.

\subsection{The influences of large-scale flows}

The driving forces for the large-scale circulation and the boundary current are different. Large-scale flows are driven by the large-scale wind field or buoyancy forcing over the open ocean, while the eastern boundary currents, although uncertainties persist, are commonly considered as an oceanic response to the coastal alongshore wind in mid-latitude EBC systems, or as buoyancy forced in high latitude marginal seas (Spall, 2004). The large-scale flow can affect and impose the variabilities of its own driving forces on the boundary variabilities. Then the slow variabilities of the large-scale phenomena will be found in coastal long-time observations.

Coastal phenomena that cannot be explained by local forcings are often documented (Freeland et al., 2003; Hickey et al., 2006; Schwing et al., 2006). For example, in the California Current System (CCS), Murphree et al. (2003) reported a cold fresh anomaly extending from Vancouver Island to southern California during summer to fall in 2002. This anomaly is associated with increased equatorward transport in the CCS and higher surface productivity in the northern CCS. One of the proposed causes is the enhanced North Pacific Current (NPC), which flows eastward with a diffluent structure near the eastern boundary. Schwing et al. (2006) found a delayed coastal upwelling in the northern California Current during 2005. They concluded that tropical El Niño events may lead to unusually weak coastal upwelling, but they are not the

only cause; the regional atmospheric forcing associated with the position, strength, and timing of the North Pacific High may also determine the upwelling. We need to include the large-scale flow influences to better predict the coastal variabilities.

However, no study has considered the dynamical mechanisms governing the interaction between a large-scale interior circulation with an EBC. In the second half of this thesis, we start the study again with a simple model. The stability of an EBC 
with a superposed large-scale flow is investigated in both the linear and nonlinear regime. The discussion only focuses on the influence of the large-scale flow on the stability of the EBC.

In the linear regime, the horizontal advection is the main influence of the largescale flow on the instabilities of an EBC. The horizontal advection can be decomposed into the zonal and meridional directions. The eastward zonal PV advection has a stabilizing effect. The meridional advection affects the unstable modes mainly through the Doppler effect. The large-scale flow can accelerate or retard the perturbation signal to introduce a meridional non-uniformity of the boundary current stability. A global mode can be established with a peak at a preferred location once parameters of the system satisfy conditions related to the strength of the large-scale flow relative to the perturbation group velocities.

Unstable flows can be classified into two categories, convectively unstable and absolutely unstable, depending on the system's long-time local response to perturbations. If the impulse response of a flow becomes unbounded for large time at all points, the flow is called absolutely unstable. Otherwise, if the impulse response decays to zero for a large time at all points, the flow is called convectively unstable. The existence of an absolutely unstable region is important for the emergence of the global mode.

As the large-scale circulation becomes stronger, the boundary jet switches from convectively unstable to absolutely unstable over the region where the large-scale circulation is strongly against the propagation of the signal. The global mode appears after the system acquires an absolutely unstable region. The position of the peak of the global mode depends linearly on the strength of the perturbation group velocity relative to the maximum meridional velocity of the large-scale circulation. Stronger large-scale flows can arrest the wave packet and push the peak of the global mode farther upstream.

In Chapter 5, we use the eddy kinetic energy as an index to study the influence of the large-scale flow on the stability of an EBC, and find that the influence of the 
large-scale flow on trapped unstable modes is different from that on long-radiating modes.

The large-scale flow can affect the trapped unstable mode by modifying the mean structure of the boundary current. A modified boundary current leads to a meridional non-homogeneity in the local stability property. This contradicts the linear assumption, in which the boundary current is assumed to be fixed and cannot be changed by the large-scale flow. With the parameters used in Chapter 5, the modified boundary current becomes more unstable over the southern domain but stable over the northern domain. The localized maximum and minimum in growth rates lead to non-homogeneity in the EKE field. The maximum EKE occurs downstream of the most unstable region because of the instabilities' convective characteristics.

The large-scale flow also influences the structures of the trapped unstable perturbations. It affects the trapped modes through the Doppler effect, as discussed in the linear theory. The unstable mode becomes shorter over the northern domain being decelerated by the gyre, and longer over the southern domain being accelerated. The shortened waves are elongated again travelling from north to south.

The alteration of the trapped modes by the large-scale flow leads to an indirect influence of the large-scale flow on the long radiating modes. The long radiating modes that appear in the nonlinear simulations are not fully self-sustained, but rather triggered and partially maintained by the trapped modes through nonlinear triadresonance. The efficiency of the nonlinear energy transfer depends on the amplitude of the trapped modes and also the phase relation of the wave-triad. The Doppler effect of the large-scale flow leads to shortened and elongated perturbations over the upstream and downstream regions, where the originally resonant triad goes out of phase and the nonlinear resonance decreases. The minimum phase shift occurs around the stagnation points of the gyres. There are two such stagnation points for a double-gyre flow, one is at the mid-latitude confluent region, and another is at the diffluent region at the southern and northern boundaries. However, the positive linear growth rates, which represent the efficiency of the energy transfer from the 
mean to the unstable modes, diminish both in amplitude and wavenumber range at the stagnation point of the confluent part of the double-gyre (at the northern and southern edges of the double-gyre), but increase at the stagnation point of the

diffluent part of the double-gyre (mid-latitude near the boundary). The differences between these two points are due to the zonal velocities of the large-scale circulation. The eastward flow compresses the boundary current inducing larger growth rates. The opposite is true for the westward flow. The combined effect causes the EKE maximum of the long radiating modes to reside in the middle of the domain near the boundary. The long radiating mode becomes more energetic following the increase of nonlinearity. The EKE of the long radiating mode can surpass the trapped modes and dominate in the total EKE field.

We have started from a simple model, demonstrating the importance of the largescale flow in modulating the boundary current instabilities. We do not expect the meridional EKE structure found here to be universal, because different boundary current profiles would certainly yield different sets of growth rates and phase/group velocities for unstable modes. However, the same mechanism found here can shed light on the phenomena in other more complex dynamical systems.

\subsection{Discussion}

This thesis systematically investigates the instabilities of an EBC with and without large-scale flow influence. Even within the simple barotropic quasi-geostrophic framework here used, the parameter space is rather wide, and is far from being fully explored. There are uncertainties, unsolved questions, and other factors worth future study.

The relationship between the phase speed (group velocity) and beta is identified but not fully solved. An increase of beta leads to an increase of the phase speed of unstable modes in a WBC but a decrease in an EBC. Previous studies based on a piecewise constant velocity profile also show the same phenomenon without 
giving an explanation. It can not simply explained by Rossby wave dynamics. More complex process related to the eigenfunction structures should be considered. It would be worth investigating whether this is a universal property regardless of the specific velocity profile. The relationship between beta and the group velocity is newly identified in this study using the spatial instability analysis. The mechanism remains, however, unexplained.

This study shows that an EBC supports fewer unstable modes than a WBC, while Hristova et al. (2008) conclude the opposite. If the difference is the consequence of the different velocity profiles used, then no general conclusion can be made on this point. The difference maybe because some marginally unstable modes over the longwave end are not resolved in our calculation. Including beta decreases the phase speed of the unstable modes. As a result, the critical layer that exists inside the boundary current in the limit $c_{i} \rightarrow 0$ may constitute a problem for the eigenvalue calculations. It is necessary to carefully examine the streamfunctions around the neighborhood of the critical layer, which is not done in this study. There is no clear analytical stability boundary defined for a meridional current. The infinitesimal growth rates over the longwave end, if they exist, can be caused by the significant radiation. For a non-radiating mode, the size of the growth rate is equivalent to the amount of the energy drawn from the mean by the Reynolds stress, $-u v V_{x}$. However, for a radiating mode, the energy radiation could be as strong as the energy input. The radiating process could be so fast that the energy drawn from the mean by the perturbation will be radiated away instantly, giving an infinitesimal growth rate. If this is true, it would be very important to identify these marginally unstable, radiating modes, because they would play important roles in the nonlinear dynamics acting efficiently to transport energy into the far field.

The linear study of the influence of the large-scale flow is constrained within a small parameter space. Most of the discussions concern the meridional development of the perturbations. The changes of their zonal structures are not investigated. However, the zonal structure is important in discussing the energy radiation. Only 
one real frequency is considered in the WKB analysis, and that frequency corresponds to a trapped mode. As a consequence, the large-scale flow influence on radiating modes has not been studied.

Although we believe that the meridional structure of the EKE of the long radiating mode is fairly explained, other factors could also be important. As shown in the linear theory, longer meridional waves are more able to radiate. The long meridional structure of perturbations can be generated by the downstream stretching effect of the double-gyre circulation. As a result, the originally trapped perturbations can be elongated to reach the wavelength of radiating modes and then radiate. This effect can be studied in the linear context by considering the zonal structures of the unstable modes.

The parameters used in this study in setting up the boundary current are within a reasonable range for a mid-latitude EBC system. The EBC with these parameters constitutes a weakly nonlinear system. Consequently, the nonlinear energy transfer is a very slow process, and because of this we can clearly elucidate the mechanisms. However, this process may not be important if the EBC is always weakly nonlinear because the boundary current may have already changed long before the nonlinear process can energize the long radiating modes.

Several phenomena not considered here might also be important for EBC stability and energy radiation. Baroclinicity can become important for increasing the nonlinearity of the system by adding an additional energy source. What are the characteristics of the radiating baroclinic instabilities? Do they obey similar mechanisms so one could extend the previous study of the baroclinic instability of a meridional boundary current (Hristova et al., 2008) by considering a continuous velocity profile with a vertical shear, and also by nonlinear simulations? Furthermore, what are the radiating characteristics of a highly nonlinear EBC with isolated eddies or turbulence embedded? The radiated energy could come from the inverse cascade of turbulence. If the inverse cascade is a very important process for the energy budget of an EBC, the beta effect can also become important by radiating the energy of the larger scale 
perturbations away from the coastal region. Although it does not destroy energy, the radiation process can drain energy out of a local system. However, most of the coastal models are on an $f$ plane without considering the process of the energy radiation.

The baroclinicity in the ocean interior is also important in modifying the radiating instabilities. The wave propagation is often constrained by geostrophic contours. In this barotropic study, the geostrophic contours are simply $\beta y$ because the large-scale flow is weakly nonlinear. No significantly curved wave rays are evident. However, a baroclinic interior gyre associates with sloped isopycnals, which will modify the PV contours. The instabilities radiated from an EBC are expected to show different behaviours when they propagate westward depending on their wave characteristics, such as the baroclinic structures. One can expect that higher baroclinic modes are more easily advected by the large-scale flows. If so, one unstable boundary region can emit radiating instabilities into different directions. The influence of the large-scale flows become much more complex. The geostrophic contours in the ocean interior can also be altered by a bottom topography. The bottom topography in the interior can then influence the wave rays emitted from an EBC by the radiating instabilities. Furthermore, a sloping bottom topography within the boundary region can also be important, as discussed in, by modifying the vertical structure of the boundary current in a baroclinic environment (Spall, 2010), and by localizing instabilities.

This thesis studied the linear and nonlinear instabilities of an eastern boundary current, identified the mechanism that governs the nonlinear excitation of radiating instabilities, and showed that the large-scale gyres induce meridional structures of perturbation kinetic energy along the boundary through modifying boundary current structure and accelerating/decelerating perturbations. These mechanisms are rather generic, and expected to remain relevant even in physically more complex systems. 


\section{Appendix A}

\section{Numerical model}

The one-layer quasi-geostrophic model on a beta plane is used in this thesis. The model equation is

$$
\frac{\partial}{\partial t} q+J(\psi, q+\beta y)=\mathcal{F}+\nabla \cdot A_{h} \nabla q
$$

where $q, \psi, \beta, \mathcal{F}, A_{h}$ represent the relative vorticity, streamfunction, meridional gradient of the Coriolis parameter, and lateral friction coefficient, respectively. The operator $J$ is the Jacobian with respect to $x$ and $y, J(A, B)=A_{x} B_{y}-A_{y} B_{x}$.

All simulations are calculated in meridional channels, extending from 0 to $W_{x}$ in the zonal direction and from 0 to $W_{y}$ in the meridional direction. The channel is north-south periodic, with solid walls along the eastern and western boundaries. The boundary conditions on the solid walss are slip $(q=0)$ and a no-flux $\left(\partial_{x}(u q)=0\right)$. With these boundary conditions, $q$ and $\psi$ can be expanded in $\sin (m \pi x) \exp \left(i n \ell_{0} y\right)$ series, where $\ell_{0}=2 \pi / W_{y}, m=0 \cdots N_{x}$, and $n=0 \cdots N_{y}$, with $N_{x}$ and $N_{y}$ respectively representing the number of grid points in $x$ and $y$. The forcing $\mathcal{F}$ can be calculated following the Sverdrup relation, $\mathcal{F}=\beta \bar{\psi}_{x}+\nabla \cdot A_{h} \nabla \nabla^{2} \bar{\psi}$, where $\bar{\psi}$ is the desired mean state.

At every time step, $q$ is first inverted to $\psi$ using an FFT in the meridional direction and a sine transform in the zonal direction. The velocities are then calculated from the streamfunction, $u=-\psi_{y}$ and $v=\psi_{x}$. The Jacobian and the lateral friction terms 
are then calculated in the flux form, $-J(\psi, q+\beta y)+\nabla \cdot A_{h} \nabla q=\partial_{x}\left(-u q+A_{h} \partial_{x} q\right)+$ $\partial_{y}\left(-v(q+\beta y)+A_{h} \partial_{y} q\right)$, using the centered difference scheme on a $\mathrm{C}$ grid. Time stepping starts with a Euler step and then continues with a second-order AdamsBashforth scheme.

The linear version of this model can be used to calculate the growth rate curve for a specified velocity profile. The linearized equation is

$$
\frac{\partial}{\partial t} q^{\prime}+J\left(\bar{\psi}, q^{\prime}\right)+J\left(\psi^{\prime}, \bar{q}+\beta y\right)=\nabla \cdot A_{h} \nabla q^{\prime},
$$

where the overbar represents the basic state, and the prime represents the perturbation. The growth rate of a specific mode, say the mode with a meridional wavenumber $n \ell_{0}$, is calculated in two steps. First the linearized model is integrated with a Fourier mode initialization, $\phi(x) \exp \left(i n \ell_{0} y\right)$, where $\phi(x)$ is an arbitrary and infinitesimal function. Then the growth rate is solved by least-square fitting of the time series of the perturbation kinetic energy to an exponential curve. 


\section{Appendix B}

\section{Eigenvalue problem}

When a current is purely meridional, $\bar{v}(x)$, we can look for trigonometric normal mode solutions for the perturbations. Substituting the normal mode solution $\psi=$ $\phi(x) \exp (i l(y-c t))$ into the linearized barotropic vorticity equation $(2.7)$, we get a generalized eigenvalue problem:

$$
L \phi=c M \phi
$$

in which

$$
\begin{aligned}
L & =\bar{v}\left(\partial_{x x}-l^{2}\right)+\frac{\beta}{i l} \partial_{x}-\bar{v}_{x x} \\
M & =\partial_{x x}-l^{2} .
\end{aligned}
$$

The partial derivative in $x$ is discretized using a centered difference scheme, with boundary condition $\phi=0$ at solid walls for east-west bounded cases. Using $N$ to denote the number of grid points, operator $L$ and $M$ become $N$ by $N$ matrices. The generalized eigenvalue problem is then solved using the program eig in Matlab. To ensure that the eigensolutions are not sensitive to the discretization scheme.This is the band matrix method, the forward and backward difference schemes are also used.

For a meridional current in a north-south periodic and east-west semi-infinite domain, the above band matrix method becomes difficult because the radiation boundary condition involves the zonal wavenumber $k$, which is not known a priori. The 
shooting method is then used to solve the eigenvalue problem.

The radiation boundary condition is

$$
\phi_{x}-i k_{1,2} \phi=0
$$

where $i$ is the imaginary unit, and $k_{1,2}$ is the zonal wavenumber satisfying the free Rossby wave dispersion relation $l c=\frac{-\beta k}{k^{2}+l^{2}}$. There are two $k$ solutions for each pair of $(l, c)$. One must chose the $k$ that corresponds to a structure of spatial decay at infinity, i.e., a westward decaying structure for the case with an eastern boundary current, or an eastward decaying structure for the case with a western boundary current.

Assume the problem concerns an eastern meridional boundary current with a wall boundary to the east and a radiation boundary condition to the west, in the domain $x=-L$ to $x=0$. Given a real meridional wavenumber $l$, we make a first guess of the complex eigenvalue $c$, then integrate the stability equation 2.7 from the eastern wall westward to get the value of $\left.\phi\right|_{-L}$ and $\left.\phi_{x}\right|_{-L}$ at $x=-L$. Without loss of generality, the boundary conditions are assumed to be $\phi=0$ and $\phi_{x}=1$ at $x=0$. The discrepancy function at $x=-L$ is $D(c)=\left.\phi_{x}\right|_{-L}-\left.\mathrm{i} k \phi\right|_{-L}$. The Newton-Raphson method is used to minimize the discrepancy function and find the eigenvalue $c$. Note that the variables are complex. Their real and imaginary parts must be treated separately. 


\section{Appendix C}

\section{Matrix perturbation}

Starting with this stability equation:

$$
\left(V\left(\partial_{x x}-l^{2}\right)+\frac{\beta}{i l} \partial_{x}-V_{x x}\right) \phi=c\left(\partial_{x x}-l^{2}\right) \phi
$$

rearranging it gives

$$
\begin{aligned}
\left(V\left(\partial_{x x}-l^{2}\right)-V_{x x}\right) \phi & =c\left(\partial_{x x}-l^{2}\right) \phi+i \beta * l^{-1} \partial_{x} \phi \\
M_{1} \phi & =c M_{2} \phi+\beta M_{3} \phi .
\end{aligned}
$$

Assume $\beta \ll O(1)$, so that

$$
\phi_{n}=\phi_{0 n}+\beta \phi_{1 n}+\beta^{2} \phi_{2 n}+O\left(\beta^{3}\right), c_{n}=c_{0 n}+\beta c_{1 n}+\beta^{2} \phi_{2 n}+O\left(\beta^{3}\right) .
$$

Then the equations for zeroth, first and second order are

$$
\begin{aligned}
& \mathcal{O}(0): \quad\left(M_{1}-c_{0 n} M_{2}\right) \phi_{0 n}=0 \\
& \mathcal{O}(0): \phi_{0 n}^{(l) H}\left(M_{1}-c_{0 n} M_{2}\right)=0 \\
& \mathcal{O}(\beta):\left(M_{1}-c_{0 n} M_{2}\right) \phi_{1 n}=c_{1 n} M_{2} \phi_{0 n}+M_{3} \phi_{0 n} \\
& \mathcal{O}\left(\beta^{2}\right):\left(M_{1}-c_{0 n} M_{2}\right) \phi_{2 n}=c_{1 n} M_{2} \phi_{1 n}+c_{2 n} M_{2} \phi_{0 n}+M_{3} \phi_{1 n}
\end{aligned}
$$


where $\phi_{0 n}^{(l) H}$ is the conjugate transpose of the n-th left eigenvector $\phi_{0 n}^{(l)}$.

The order one equation is

$$
\left(M_{1}-c_{0 n} M_{2}\right) \phi_{1 n}=c_{1 n} M_{2} \phi_{0 n}+M_{3} \phi_{0 n}
$$

Left multiplying $\phi_{0 k}^{(l) H}$ of the first order equation gives

$$
\phi_{0 k}^{(l) H}\left(M_{1}-c_{0 n} M_{2}\right) \phi_{1 n}=c_{1 n} \phi_{0 k}^{(l) H} M_{2} \phi_{0 n}+\phi_{0 k}^{(l) H} M_{3} \phi_{0 n} .
$$

To get the value of $c_{1 n}$, let $k=n$, then the left-hand-side is zeros by definition, and the second term on the left-hand-size is also zeros as $M_{3}$ is $i l^{-1} \partial_{x}$, and $\phi_{0 k}^{(l) H}$ has the same property of symmetry as $\phi_{0 n}$, either symmetric or antisymmetric. Then we get

$$
c_{1 n}=\phi_{0 k}^{(l) H} M_{3} \phi_{0 n}=0
$$

which means we have to seek a higher order correction.

With the knowledge of $c_{1 n}=0$, we can get the value of $\phi_{1 n}$. The first order equation is

$$
\begin{aligned}
& \left(M_{1}-c_{0 n} M_{2}\right) \phi_{1 n}=c_{1 n} M_{2} \phi_{0 n}+M_{3} \phi_{0 n} \\
& \left(M_{1}-c_{0 n} M_{2}\right) \phi_{1 n}=M_{3} \phi_{0 n} .
\end{aligned}
$$

As $\left(M_{1}-c_{0 n} M_{2}\right)$ is one singular matrix, we can use the least-square/SVD method to get $\phi_{1 n}$ from the zeroth order results. Thus

$$
M=\left(M_{1}-c_{0 n} M_{2}\right)=U \wedge V^{H}
$$

where $\wedge=\operatorname{diag}\left(c_{0 i}\right), i=1 . . N$

We have

$$
\phi_{1 n}=V \wedge^{-1} U^{H} M_{3} \phi_{0 n}
$$


$\wedge^{-1}=\operatorname{diag}\left(1 / c_{0 i}\right)$, if $c_{0 i} \neq 0 ; \wedge^{-1}=0$ otherwise.

If we want to examine one specific mode's influence on another mode, say sinuous mode on varicose mode, we can left multiply $\phi_{1 k}^{(l) H}$ of the first order equation to get

$$
\phi_{0 k}^{(l) H}\left(M_{1}-c_{0 n} M_{2}\right) \phi_{1 n}=\phi_{0 k}^{(l) H} M_{3} \phi_{0 n} ; k \neq n .
$$

As $\phi_{0 k}^{(l) H}\left(M_{1}-c_{0 k} M_{2}\right)=0$, the above equation can be written as

$$
\left(c_{0 k}-c_{0 n}\right) \phi_{0 k}^{(l) H} M_{2} \phi_{1 n}=\phi_{0 k}^{(l) H} M_{3} \phi_{0 n}
$$

and we get

$$
\phi_{1 n k}=\frac{M_{2}^{-1} M_{3} \phi_{0 n}}{c_{0 k}-c_{0 n}}
$$

$\phi_{1 n k}$ represents influence of the $k$-th mode modified by the operator $M_{3}$ on the $n$-th mode. We will see from the expression of the second order correction $c_{2 n}$ that the relative magnitude of $c_{0 k}$ and $c_{0 n}$ alters the sign of $c_{2 n}$.

With the information of the first order, we can get the second order correction.

$$
\left(M_{1}-c_{0 n} M_{2}\right) \phi_{2 n}=c_{2 n} M_{2} \phi_{0 n}+M_{3} \phi_{1 n}
$$

Following the same procedure, we get

$$
c_{2 n}=-\frac{\phi_{0 n}^{(l) H} M_{3} \phi_{1 n}}{\phi_{0 n}^{(l) H} \phi_{0 n}} .
$$

If $\phi_{1 n}$ is calculated by the least-square method, we get the overall contribution of the zero-th order eigensolutions. If $\phi_{1 n k}$ is used, then we get the information about how different modes interact with each other, among which we are interested in the varicose and the sinuous mode interaction. 
THIS PAGE INTENTIONALLY LEFT BLANK 


\section{Bibliography}

Allen, J., L. Walstad, and P. Newberger, 1991: Dynamics of the coastal transition zone jet. 2. nonlinear finite amplitude behavior. Journal of Geophysical Research, 96, $14995-15016$.

Barth, J., 1989a: Stability of a coastal upwelling front 1. model development and a stability theorem. Journal of Geophysical Research, 94 (C8), 10 844-10.

Barth, J., 1989b: Stability of a coastal upwelling front 2. model results and comparison with observations. Journal of Geophysical Research, 94 (C8), 10 857-10.

Barth, J., 1994: Short-wavelength instabilities on coastal jets and fronts. Journal of Geophysical Research, 99 (16), 095-16.

Barton, E., et al., 1998: The transition zone of the canary current upwelling region. Progress in Oceanography, 41 (4), 455-504.

Batteen, M., 1997: Wind-forced modeling studies of currents, meanders, and eddies in the california current system. Journal of geophysical research, 102 (C1), 985-1010.

Batteen, M. and M. Rutherford, 1990: Modelling studies of eddies in the leeuwin current: the role of thermal forcing. Journal of Physical Oceanography, 20 (9), 1484-1520.

Berloff, P. and J. McWilliams, 1999: Quasigeostrophic dynamics of the western boundary current. Journal of Physical Oceanography, 29 (10), 2607-2634.

Bernstein, R., L. Breaker, and R. Whritner, 1977: California current eddy formation: ship, air, and satellite results. Science, 195 (4276), 353.

Bers, A., 1975: Linear waves and instabilities. Plasma Physics, 117-225.

Bickley, W., 1937: The plane jet. Phil. Mag, 23 (7), 727-731.

Bracco, A., J. Pedlosky, and R. Pickart, 2008: Eddy formation near the west coast of greenland. Journal of Physical Oceanography, 38 (9), 1992-2002.

Bretherton, F., 1964: Resonant interactions between waves. the case of discrete oscillations. Journal of Fluid Mechanics, 20 (03), 457-479. 
Briggs, R., 1964: Electron-stream interaction with plasmas. Research monograph, M.I.T. Press, URL http://books.google.com/books?id=YgpXPQAACAAJ.

Brink, K., 1987: Coastal ocean physical processes. Reviews of Geophysics, 25 (2), 204-216.

Brink, K. and T. Cowles, 1991: The coastal transition zone program. Journal of Geophysical Research, 96 (C8), 14637-14.

Chang, E. and I. Orlanski, 1993: On the dynamics of a storm track. Journal of the Atmospheric Sciences, 50 (7), 999-999.

Craik, A., 1971: Non-linear resonant instability in boundary layers. Journal of Fluid Mechanics, 50 (02), 393-413.

Cresswell, G. and T. Golding, 1980: Observations of a south-flowing current in the southeastern indian ocean. Deep Sea Research Part A. Oceanographic Research Papers, 27 (6), 449-466.

Davis, R., 1985a: Drifter observations of coastal surface currents during code: The method and descriptive view. Journal of Geophysical Research, 90 (C3), 47414755 .

Davis, R., 1985b: Drifter observations of coastal surface currents during code: The statistical and dynamical views. Journal of Geophysical Research, 90 (C3), 47564772 .

Dierckx, P., 1995: Curve and surface fitting with splines. Oxford University Press, USA.

Domingues, C., S. Wijffels, M. Maltrud, J. Church, and M. Tomczak, 2006: Role of eddies in cooling the leeuwin current. Geophysical research letters, 33 (5), L05 603.

Fantini, M. and K. Tung, 1987: On radiating waves generated from barotropic shear instability of a western boundary current. Journal of Physical Oceanography, 17 (8), 1304-1308.

Flament, P., L. Armi, and L. Washburn, 1985: The evolving structure of an upwelling filament. Journal of Geophysical Research, 90 (11), 765-11.

Flierl, G. and V. Kamenkovich, 1975: Gulf stream meandering and gulf stream ring eddy production mechanisms. MIT Press, 115-118 pp.

Fox-Kemper, B., 2003: Eddies and friction: removing of vorticity from the winddriven gyre. Ph.D. thesis, MIT/WHOI Joint Program in Oceanography.

Freeland, H., G. Gatien, A. Huyer, and R. Smith, 2003: Cold halocline in the northern california current: An invasion of subarctic water. Geophys. Res. Lett, 30 (3), 1141. 
Gaster, M., 1962: A note on the relation between temporally-increasing and spatiallyincreasing disturbances in hydrodynamic stability. Journal of fluid mechanics, 14 (02), 222-224.

Godfrey, J. and T. Golding, 1981: The sverdrup relation in the indian ocean, and the effect of pacific-indian ocean throughflow on indian ocean circulation and on the east australian current. Journal of Physical Oceanography, 11, 771-779.

Godfrey, J. and K. Ridgway, 1985: The large-scale environment of the polewardflowing leeuwin current, western australia: longshore steric height gradients, wind stresses and geostrophic flow. Journal of Physical Oceanography, 15 (5), 481-495.

Griffiths, R. and P. Linden, 1981: The stability of buoyancy-driven coastal currents. Dynamics of Atmospheres and Oceans, 5 (4), 281-306.

Haidvogel, D., A. Beckmann, and K. Hedström, 1991: Dynamical simulations of filament formation and evolution in the coastal transition zone. Journal of Geophysical Research, 96 (C8), 15017-15.

Haney, R., R. Hale, and D. Dietrich, 2001: Offshore propagation of eddy kinetic energy in the california current. Journal of Geophysical Research, 106 (C6), 11 70911.

Harrison, D. and A. Robinson, 1979: Boundary-forced planetary waves: A simple model mid-ocean response to strong current variability. Journal of Physical Oceanography, 9, 919-929.

Herbert, T., 1988: Secondary instability of boundary layers. Annual review of fluid mechanics, 20 (1), 487-526.

Hickey, B., A. MacFadyen, W. Cochlan, R. Kudela, K. Bruland, and C. Trick, 2006: Evolution of chemical, biological, and physical water properties in the northern california current in 2005: Remote or local wind forcing? Geophysical Research Letters, 33 (22), L22S02.

Hristova, H. G., J. Pedlosky, and M. A. Spall, 2008: Radiating instability of a meridional boundary current. Journal of Physical Oceanography., 38, 2294-2307.

Huerre, P. and P. Monkewitz, 1990: Local and global instabilities in spatially developing flows. Annual Review of Fluid Mechanics, 22 (1), 473-537.

Huyer, A., et al., 1991: Currents and water masses of the coastal transition zone off northern california, june to august 1988. Journal of Geophysical Research, 96.

Ierley, G. and W. Young, 1991: Viscous instabilities in the western boundary layer. Journal of Physical Oceanography, 21 (9), 1323-1332. 
Ikeda, M. and W. Emery, 1984: Satellite observations and modeling of meanders in the california current system off oregon and northern california. Journal of Physical Oceanography, 14 (9), 1434-1450.

Kamenkovich, I. and J. Pedlosky, 1996: Radiating instability of nonzonal ocean currents. Journal of Physical Oceanography, 26 (4), 622-643.

Kamenkovich, I. and J. Pedlosky, 1998a: Radiation of energy from nonzonal ocean currents, nonlinear regime. part i: Single wave development. Journal of Physical Oceanography, 28 (9), 1661-1682.

Kamenkovich, I. and J. Pedlosky, 1998b: Radiation of energy from nonzonal ocean currents, nonlinear regime. part ii: Interactions between waves. Journal of Physical Oceanography, 28 (9), 1683-1701.

Kelly, K., 1985: The influence of winds and topography on the sea surface temperature patterns over the northern california slope. Journal of Geophysical Research, 90 (C6), 11 783-11.

Kelly, K., R. Beardsley, R. Limeburner, K. Brink, J. Paduan, and T. Chereskin, 1998: Variability of the near-surface eddy kinetic energy in the california current based on altimetric, drifter, and moored current data. Journal of Geophysical research, 103 (C6), $13067-13$.

Kosro, P. and A. Huyer, 1986: Ctd and velocity surveys of seaward jets off northern california, july 1981 and 1982. Journal of Geophysical Research, 91, 7680-7690.

Kuo, H., 1949: Dynamic instability of two-dimensional nondivergent flow in a barotropic atmosphere. Journal of Meteorology, 6 (2), 105-122.

Kuo, H., 1973: Dynamics of quasigeostrophic flows and instability theory. Advances in Applied Mechanics, 13, 247-330.

Legeckis, R. and G. Cresswell, 1981: Satellite observations of sea-surface temperature fronts off the coast of western and southern australia. Deep Sea Research Part A. Oceanographic Research Papers, 28 (3), 297-306.

Levitus, S. and R. Gelfeld, 1992: National oceanographic data center inventory of physical oceanographic profiles. global distributions by year for all countries.

Lipps, F., 1962: The barotropic stability of the mean winds in the atmosphere. Journal of Fluid Mechanics, 12 (03), 397-407.

Liu, Z., 1999: Planetary wave modes in the thermocline: Non-doppler-shift mode, advective mode and green mode. Quarterly Journal of the Royal Meteorological Society, 125 (556), 1315-1339. 
Lutjeharms, J. and J. Meeuwis, 1987: The extent and variability of south-east atlantic upwelling. South African Journal of Marine Science, 5 (1), 51-62.

Lutjeharms, J., F. Shillington, and C. Rae, 1991: Observations of extreme upwelling filaments in the southeast atlantic ocean. Science, 253 (5021), 774.

Malanotte-Rizzoli, P., D. Haidvogel, and R. Young, 1987: Numerical simulation of transient boundary-forced radiation. i: The linear regime. Journal of Physical Oceanography, 17 (9), 1439-1457.

Marchesiello, P., J. McWilliams, and A. Shchepetkin, 2003: Equilibrium structure and dynamics of the california current system. Journal of Physical Oceanography, $33(4), 753-783$.

Maslowe, S., 1991: Barotropic instability of the bickley jet. Journal of Fluid Mechanics, 229, 417-426.

Maximenko, N., B. Bang, and H. Sasaki, 2005: Observational evidence of alternating zonal jets in the world ocean. Geophys. Res. Lett, 32, L12 607.

McCreary, J., Y. Fukamachi, and P. Kundu, 1991: A numerical investigation of jets and eddies near an eastern ocean boundary. Journal of Geophysical Research, 96 (C2), 2515-2534.

McCreary, J., S. Shetye, and P. Kundu, 1986: Thermohaline forcing of eastern boundary currents: with application to the circulation off the west coast of australia. Journal of Marine Research, 44 (1), 71-92.

McIntyre, M. and M. Weissman, 1978: On radiating instabilities and resonant overreflection. Journal of Atmospheric Sciences, 35, 1190-1196.

Meeuwis, J. and J. Lutjeharms, 1990: Surface thermal characteristics of the angolabenguela front. South African Journal of Marine Science, 9 (1), 261-279.

Mittelstaedt, E., 1991: The ocean boundary along the northwest african coast: circulation and oceanographic properties at the sea surface. Progress in Oceanography, 26 (4), 307-355.

Munk, W., 1950: On the wind-driven ocean circulation. Journal of Meteorology, 7 (2), 79-93.

Murphree, T., S. Bograd, F. Schwing, and B. Ford, 2003: Large scale atmosphereocean anomalies in the northeast pacific during 2002. Geophysical Research Letters, 30 (15), 8026.

Narimousa, S. and T. Maxworthy, 1989: Application of a laboratory model to the interpretation of satellite and field observations of coastal upwelling. Dynamics of atmospheres and oceans, 13 (1-2), 1-46. 
Orszag, S. and A. Patera, 1983: Secondary instability of wall-bounded shear flows. Journal of Fluid Mechanics, 128 (-1), 347-385.

Pedlosky, J., 1977: On the radiation of meso-scale energy in the mid-ocean. Deep Sea Research, 24 (6), 591-600.

Pedlosky, J., 1987: Geophysical Fluid Dynamics. corr. 2nd ed., Springer.

Pedlosky, J., 1993: The reflection of unstable baroclinic waves and the production of mean coastal currents. Journal of Physical Oceanography, 23 (9), 2130-2135.

Phillips, O., 1960: On the dynamics of unsteady gravity waves of finite amplitude part 1. the elementary interactions. Journal of Fluid Mechanics, 9 (02), 193-217.

Pierrehumbert, R. T., 1984: Local and global baroclinic variability of zonally varying flow. Journal of the Atmospheric Sciences., 41, 2141-2162.

Ridgway, K. and S. Condie, 2004: The 5500-km-long boundary flow off western and southern australia. Journal of Geophysical Research, 109 (C4), C04017.

Schwing, F., N. Bond, S. Bograd, T. Mitchell, M. Alexander, and N. Mantua, 2006: Delayed coastal upwelling along the us west coast in 2005: A historical perspective. Geophys. Res. Lett, 33 (22), L22S01.

Shannon, L., J. Agenbag, and M. Buys, 1987: Large-and mesoscale features of the angola-benguela front. South African Journal of Marine Science, 5 (1), 11-34.

Spall, M., 1992: Rossby wave radiation in the cape verde frontal zone. Journal of Physical Oceanography, 22 (7), 796-807.

Spall, M., 2000: Generation of strong mesoscale eddies by weak ocean gyres. Journal of Marine Research, 58 (1), 97-116.

Spall, M., 2003: Islands in zonal flow. Journal of Physical Oceanography, 33 (12), 2689-2701.

Spall, M., 2004: Boundary currents and watermass transformation in marginal seas. Journal of physical oceanography, 34 (5), 1197-1213.

Spall, M., 2010: Non-local topographic influences on deep convection: An idealized model for the nordic seas. Ocean Modelling, 32 (1-2), 72-85.

Stommel, H., 1948: The westward intensification of wind-driven ocean currents. Trans. Amer. Geophys. Union, 29 (2), 202-206.

Strub, P. and C. James, 2000: Altimeter-derived variability of surface velocities in the california current system: 2. seasonal circulation and eddy statistics. Deep Sea Research Part II: Topical Studies in Oceanography, 47 (5-6), 831-870. 
Strub, P. and C. James, 2002: Altimeter-derived surface circulation in the large-scale ne pacific gyres.:: Part 1. seasonal variability. Progress in Oceanography, 53 (2-4), $163-183$.

Strub, P., P. Kosro, A. Huyer, and C. Collaborators, 1991: The nature of the cold filaments in the california current system. Journal of Geophysical Research, 96 (C8), 14743 .

Strub, P., J. Mesias, and C. James, 1995: Altimeter observations of the peru-chile countercurrent. Geophysical Research Letters, 22, 211-214.

Talley, L., 1983: Radiating barotropic instability. Journal of Physical Oceanography, 13 (6), 972-987.

Thompson, R., 1984: Observations of the leeuwin current off western australia. Journal of Physical Oceanography, 14, 623-628.

Walker, A. and J. Pedlosky, 2002: Instability of meridional baroclinic currents. Journal of Physical Oceanography., 32, 1075-1093.

Weaver, A. and J. Middleton, 1989: On the dynamics of the leeuwin current. Journal of Physical Oceanography, 19 (5), 626-648. 